Universidade de São Paulo

Instituto de Física

\title{
Estudo teórico de complexos de transferência de carga em solução
}

\author{
Fernando da Silva
}

Orientador: Profa. Dra. Kaline Rabelo Coutinho

Tese de doutorado apresentada ao Instituto de Física da Universidade de São Paulo para a obtenção do título de Doutor em Ciências.

\section{Banca Examinadora:}

Profa. Dra. Kaline Rabelo Coutinho (IF/USP)

Prof. Dr. Caetano Rodrigues Miranda (IF/USP)

Prof. Dr. Luis Gregório Godoy de Vasconcellos Dias da Silva (IF/USP)

Prof. Dr. Antônio Carlos Borin (IQ/USP)

Prof. Dr. Nelson Henrique Morgon (IQ/UNICAMP)

São Paulo

2016 


\section{FICHA CATALOGRÁFICA}

Preparada pelo Serviço de Biblioteca e Informação do Instituto de Física da Universidade de São Paulo

Silva, Fernando da

Estudo teórico de complexos de transferência de carga em solução. São Paulo, 2016.

Tese (Doutorado) - Universidade de São Paulo. Instituto de Física. Depto. de Física Geral

Orientador: Profa. Dra. Kaline Rabelo Coutinho

Área de Concentração: Física Atômica e Molecular.

Unitermos: 1. Física molecular; 2. Química quântica; 3. Estrutura eletrônica; 4. Espectroscopia; 5. Solvatação.

USP/IF/SBI-085/2016 
"It is nice to know that the computer understands the problem. But I would like to understand it too"

Eugene Wigner 



\section{Agradecimentos}

Ao CNPq pelo apoio financeiro (processo $n^{\circ}$. 158651/2011-1).

Ao Laboratório de Computação Científica Avançada (LCCA) da USP e o convênio USP-RICE que permitiu o acesso ao Blue-Gene/P no qual foram realizadas as dinâmicas por primeiros princípios.

A minha orientadora, Profa. Dra. Kaline Coutinho.

Ao Prof. Dr. Benedito José Costa Cabral (Universidade de Lisboa) pela ajuda e orientação com as dinâmicas por primeiros princípios.

A todos do Grupo de Física Molecular e Modelagem, pela amizade, pelo apoio conversas e discussões.

Aos funcionários do departamento de Física Geral, bem como da CPG do IFUSP.

A minha família pelo apoio e a Márcia por estar ao meu lado durante o doutorado.

Finalmente, a todos que estiveram ao meu lado ao longo destes anos, que torceram, que rezaram e contribuiram para que este trabalho pudesse ser realizado. Certamente não vou ser capaz de citá-los todos aqui, mas deixo registrado meus mais sinceros sentimentos de gratidão.

Muito Obrigado a todos! 



\section{Resumo}

Neste trabalho foram estudados os complexos de transferência (CTC) de carga formados por iodeto com os derivados piridínicos $C_{4}(4 C P)^{+} I^{-}$e $C_{3} b i s(4 C P)^{2+}$ em solução. A formação de um CTC é caracterizada pelo surgimento de uma nova banda no espectro eletrônico de absorção, em solventes orgânicos como acetonitrila. Este tipo de sistema tem recebido muito interesse em diversos campos como, por exemplo, eletrônica orgânica, espectroscopia não linear, bioquímica, no ramo farmacêutico, etc.

O complexo $C_{4}(4 C P)^{+} I^{-}$é caracterizado por uma banda de transferência de carga com máximo em $421 \mathrm{~nm}$ em acetonitrila. Cálculos das propriedades eletrônicas e das energias de excitação do complexo foram realizados usando a teoria do funcional da densidade e a teoria do funcional da densidade dependente do tempo. O emprego de funcionais de troca e correlação com correções de longo alcance foi essencial para a obtenção de resultados acurados para as energias de excitação. Usando os funcionais CAM-B3LYP e $\omega$ B97X-D, associados ao modelo contínuo PCM, foi possível descrever muito bem o máximo da banda experimental.

No caso do $C_{3} b i s(4 C P)^{2+}$ foram encontradas diferentes estruturas possíveis para o complexo, que podem ser formadas pela associação de um ou dois $I^{-}$. Em todos os casos apenas um dos $I^{-}$participa da excitação, o que explica a estequiometria 1:1 observada experimentalmente. Uma visão mais aprofundada do comportamento do complexo em solução foi obtida usando a dinâmica molecular clássica. O campo de força OPLS-AA foi ajustado para reproduzir os resultados de uma dinâmica por primeiros princípios. A dinâmica clássica mostrou não haver dissociação em acetonitrila no complexo formado pelo iodeto com o $C_{3} b i s(4 C P)^{2+}$. A partir das configurações foi possível calcular a banda de absorção do complexo em excelente acordo com o resultado experimental. 



\section{Abstract}

In this work, we have studied theoretically charge-transfer complexes (CTC) formed by pyridinium derivatives with iodide. The formation of a CTC is characterized by the appearance of a new absorption band on the electronic spectra, in organic polar solvents like acetonitrile. These type of systems have recently received much interest in a broad variety of fields, for example, organic electronics, nonlinear spectroscopy, medical biochemistry, pharmaceutical industry, etc. The $C_{4}(4 C P)^{+} I^{-}$complex is characterized by the charge-transfer band with a maximum at $421 \mathrm{~nm}$ in acetonitrile.

We have used density functional theory (DFT) and time dependent density functional theory (TDDFT) to calculate electronic properties and the excitation energies of the complex. Functionals with long-range corrections were essential in describing the charge-transfer excitations. CAM-B3LYP and $\omega$ B97X-D associated with the polarizable continuum model predicts $\mathrm{CT}$ excitations in good agreement with experiment.

Our results also indicates the existence of different conformations for the complex formed by the $C_{3} b i s(4 C P)^{2+}$ with iodide. Complexes were formed by the association of one or two $I^{-}$to $C_{3} b i s(4 C P)^{2+}$, but the charge transfer excitations were calculated from only one iodide to the aromatic ring, what explain why the stoichiometry 1:1 was observed. A better description of the complex in solution was obtained using classical molecular dynamics. The OPLS-AA force field was fine-tuned to reproduce the results of a first principle molecular dynamics for the complex. No dissociation were observed. The calculated charge-transfer band using configurations sampled from molecular dynamics is in excellent agreement with experiment. 



\section{Sumário}

Lista de Abreviaturas xiii

$\begin{array}{ll}\text { Lista de Figuras } & \text { XV }\end{array}$

Lista de Tabelas $\quad$ xix

1 Introdução 1

1.1 Complexos moleculares . . . . . . . . . . . . . . . . . 3

1.2 Constante de equilíbrio . . . . . . . . . . . . . . . . 4

1.3 Complexos formados por iodeto e derivados piridínicos . . . . . . . . 5

1.4 Estudo teórico de complexos de transferência de carga . . . . . . . . . . 10

1.5 Estudo teórico de moléculas em solução . . . . . . . . . . . . . . . . . . 11

1.5.1 Abordagem supermolecular e modelos contínuos . . . . . . . . . 11

1.5.2 Dinâmica molecular . . . . . . . . . . . . . . . . . . . . . . . . 13

1.5.3 A combinação entre mecânica quântica e mecânica molecular . 14

1.6 Organização da tese . . . . . . . . . . . . . . . . . . . 17

2 Fundamentos da DFT e da TDDFT 19

2.1 Introdução . . . . . . . . . . . . . . . . . . . . . . . . . 21

2.2 O problema eletrônico . . . . . . . . . . . . . . . . . . . . . . 21

2.3 A teoria do funcional da densidade . . . . . . . . . . . . . . . 24

2.3.1 Equações de Khon-Sham . . . . . . . . . . . . . . . . . . 26

2.3.2 Aproximações para o potencial de troca-correlação . . . . . . . . 29 
2.3.3 Funcionais híbridos . . . . . . . . . . . . . . . . . . 31

2.3.4 Correções de longo alcance . . . . . . . . . . . . . . . . . 32

2.4 Solução das equações de Kohn-Sham . . . . . . . . . . . . . . . . . . . 33

2.5 Funções Base . . . . . . . . . . . . . . . . . . . . . . . 34

2.5.1 Bases gaussianas e funções de Slater . . . . . . . . . . . . . 34

2.5 .2 Bases de ondas planas . . . . . . . . . . . . . . . 36

2.6 Teoria do funcional da densidade dependente do tempo . . . . . . . . . 38

2.7 O problema dependente do tempo . . . . . . . . . . . . . . . . . . . . 39

2.8 O formalismo da TDDFT . . . . . . . . . . . . . . . . 40

2.8.1 O teorema de Runge-Gross . . . . . . . . . . . . . . . . . 40

2.8.2 O formalismo de Kohn-Sham dependente do tempo . . . . . . . 41

2.8.3 O potencial de troca-correlação dependente do tempo . . . . . . 41

2.8.4 TDDFT e resposta linear . . . . . . . . . . . . . . . . 42

2.8 .5 Formalismo . . . . . . . . . . . . . . . . . . 43

2.8.6 O cálculo das energias de excitação . . . . . . . . . . . . . . 46

2.8.7 Excitações de transferência de carga . . . . . . . . . . . . . . . 47

3 Dinâmica molecular $\quad 49$

3.1 Introdução . . . . . . . . . . . . . . . . . . . . . . . . . 51

3.2 Aproximação clássica . . . . . . . . . . . . . . . . . . . . . . 51

3.3 Dinâmica molecular de Born-Oppenheimer . . . . . . . . . . . . 52

3.3 .1 O método GPW . . . . . . . . . . . . . . . . 53

3.3 .2 Pseudopotenciais de GTH . . . . . . . . . . . . . 54

3.4 Dinâmica molecular clássica . . . . . . . . . . . . . . . . . . . . . 55

3.4.1 Condições periódicas de contorno . . . . . . . . . . . . . . 60

3.4.2 Correções de longo alcance para a interação eletrostática . . . . 61

3.5 Integração das equações de movimento . . . . . . . . . . . . . . . . 62

3.6 Dinâmica molecular e mecânica estatística . . . . . . . . . . . . . . . 62

3.7 O protocolo de simulação . . . . . . . . . . . . . . . . . . . . . 64

3.8 Procedimentos de análise . . . . . . . . . . . . . . . . . . 65

3.8 .1 RMSD . . . . . . . . . . . . . . . 6 66

3.8.2 Distribuição radial de pares . . . . . . . . . . . . . 66 
3.8 .3 Clustering . . . . . . . . . . . . . . . 67

3.8.4 Convolução do espectro UV/Vis . . . . . . . . . . . . . . . . 68

4 Estrutura eletrônica dos complexos $\quad 71$

4.1 Complexos $C_{4}(4 C P)^{+} X^{-} \ldots \ldots \ldots \ldots$. . . . . . . . . . 73

4.1 .1 Geometria de equilíbrio . . . . . . . . . . . . 73

4.1.2 Densidade de carga e análise de população . . . . . . . . . . . . 75

4.1 .3 Energias de excitação . . . . . . . . . . . . . . . . . . 79

4.1.4 Excitações em solução usando o modelo contínuo . . . . . . . . 85

4.2 Complexo $C_{3} b i s(4 C P)^{2+} I^{-} \quad \ldots \ldots \ldots \ldots$. . . . . . . . . . . 90

4.2 .1 Estruturas de equilíbrio . . . . . . . . . . . . . . . 90

4.2.2 Densidade de carga e análise de população . . . . . . . . . . . . 92

4.2 .3 Energias de excitação . . . . . . . . . . . . . . . 95

4.2 .4 Adição de um segundo iodeto . . . . . . . . . . . . . . . 98

5 Dinâmica molecular dos complexos $\quad 103$

5.1 Dinâmica molecular por primeiros princípios . . . . . . . . . . . . 105

5.1 .1 Detalhes computacionais . . . . . . . . . . . 105

5.1 .2 Dinâmica molecular do complexo em vácuo . . . . . . . . . . . . 106

5.1 .3 Espectro de absorção calculado . . . . . . . . . . . . . . . . 109

5.2 Dinâmica molecular clássica . . . . . . . . . . . . . . . . . . . 115

5.2.1 Parametrização e refinamento do campo de força . . . . . . . . 115

5.2.2 Dinâmica do $C_{3}$ bis $(4 C P)^{2+}$ com $2 I^{-}$em acetonitrila . . . . . . 121

5.2 .3 Cálculo do espectro de absorção em solução . . . . . . . . . . . 127

5.3 Extensão dos resultados do campo de força . . . . . . . . . . . . . . . . 131

5.3.1 Complexo formado pelo $C_{3}$ bis $(4 C P)^{2+}$ com $2 \mathrm{I}^{-}$em água . . . . 131

5.3.2 Complexo formado pelo $C_{8}$ bis $(4 C P)^{2+}$ com $2 \mathrm{I}^{-} \ldots \ldots$. . . . 133

6 Considerações finais $\quad 137$

$\begin{array}{lr}\text { Referências Bibliográficas } & 139\end{array}$

$\begin{array}{ll}\text { A Informação suplementar } & 169\end{array}$ 
A.1 Geometrias otimizadas . . . . . . . . . . . . . . 170

A.2 Espectro vibracional . . . . . . . . . . . . . . . . . . . . . 177

A.3 Propriedades magnéticas . . . . . . . . . . . . . . . . . 180

B Modos vibracionais via função de autocorrelação de velocidades $\quad 185$

$\begin{array}{ll}\text { C Campo de força para acetonitrila } & 189\end{array}$

C.1 Correções de longo alcance para a interação eletrostática ...... 189

D Arquivos adicionais das dinâmicas $\quad 195$

D.1 C2PK . . . . . . . . . . . . . . . . . . . . 195

D.2 Gromacs . . . . . . . . . . . . . . . . . . . . 198

D.2.1 Topologia do $C_{3} b i s(4 C P)^{2+}$. . . . . . . . . . . . . . 198

D.2.2 Topologia da acetonitrila . . . . . . . . . . . . . 206

D.2.3 Arquivo de entrada para a dinâmica clássica - GROMACS . . . 207 


\title{
Lista de Abreviaturas
}

\author{
AIMD $\quad$ Ab Initio Molecular Dynamics \\ AMBER Assisted Model Building with Energy Refinement \\ BOMD Born-Oppenheimer Molecular Dynamics \\ CC Coupled Cluster \\ CHARMM Chemistry at HARvard Macromolecular Mechanics \\ CHELPG CHarges from Electrostatic Potentials using a Grid-based method \\ CI Configuration Interaction \\ CPMD Car Parrinello Molecular Dynamics \\ CTC Charge Transfer Complexes \\ DFT Density Functional Theory \\ EDA Electron donor-acceptor \\ GGA Gradient Generalized Aproximation \\ GPW Gaussian and Plane Waves \\ GROMOS GROningen MOlecular Simulation \\ GTH Pseudo potenciais de Goedecker, Teter e Hutter \\ GTO Gaussian Type Orbitals
}




\begin{tabular}{|c|c|}
\hline $\mathrm{HF}$ & Hartre-Fock \\
\hline HK & Hohenberg-Kohn \\
\hline HOMO & Highest Occupied Molecular Orbital \\
\hline $\mathrm{KS}$ & Kohn-Sham \\
\hline $\mathrm{LCAO}$ & Linear Combination of Atomic Orbitals \\
\hline LDA & Local Density Aproximation \\
\hline LUMO & Lowest Unoccupied Molecular Orbital \\
\hline MD & Molecular Dynamics \\
\hline MDDF & Minimum Distance Distribution Function \\
\hline MEP & Molecular Electrostatic Potential \\
\hline MM & Molecular Mechanics \\
\hline NPA & Natural Population Analysis \\
\hline OPLS & Optimized Parameter for Liquid Simulation \\
\hline QM & Quantum Mechanics \\
\hline $\mathrm{QM} / \mathrm{MM}$ & Quantum Mechanics/ Molecular Mechanics \\
\hline PCM & Polarizable Continuum Model \\
\hline PME & Particle-Mesh Ewald \\
\hline $\mathrm{RDF}$ & Radial Distribution Function \\
\hline RMSD & Root Mean Square Deviation \\
\hline SCRF & Self Consistent Reaction Field \\
\hline STO & Slater Type Orbitals \\
\hline TD & Time Dependent \\
\hline
\end{tabular}




\section{Lista de Figuras}

1.1 Representação esquemática dos derivados piridínicos . . . . . . . . . . . 6

1.2 Espectro de absorção experimental do CTC medido em acetonitrila . . 7

1.3 Ajuste experimental para a determinação estequiométrica do CTC $\quad$. 9

1.4 Representação esquemática do equilíbrio químico proposto, formação do complexo sanduíche . . . . . . . . . . . . . . . . . 9 9

3.1 Ilustração dos conceitos de distância, ângulo e diedro . . . . . . . . . . 58

4.1 Geometrias de equilíbrio dos complexos com iodeto e brometo . . . . . 74

4.2 Mapas de densidade eletrônica para os complexos com iodeto e brometo 77

4.3 Distribuição eletrônica nos complexos com iodeto e brometo . . . . . . 78

4.4 Orbitais moleculares de fronteira . . . . . . . . . . . . . . . 82

4.5 Representação das estruturas de mínima energia obtidas em solução 87

4.6 Representação das estruturas de mínima energia . . . . . . . . . . . . . . 91

4.7 Varredura dos ângulos diedrais do $C_{3}$ bis $(4 C P)^{2+} \ldots \ldots$. . . . . . . . 91

4.8 Mapa de diferença de densidade eletrônica do complexo $C_{3} b i s(4 C P)^{2+} I^{-} 93$

4.9 Districuição de cargas naturais do complexo $C_{3}$ bis $(4 C P)^{2+} I^{-} \quad$. . . . . 94 
4.10 Orbitais Moleculares de fronteira para o $C_{3} b i s(4 C P)^{2+} I^{-} \ldots \ldots$. . . . 97

4.11 Comparação entre as energias de excitação calculadas e o espectro experimental . . . . . . . . . . . . . . . . . . 97

4.12 Representação estrutural das geometrias para o complexo com dois iodetos 98

4.13 Orbitais Moleculares de KS para o complexo com dois iodetos . . . . . 100

5.2 Evolução temporal da temperatura e energia potencial . . . . . . . . . 106

5.3 Evolução temporal da Distância C ․ I . . . . . . . . . . . . . 107

5.4 Transformada de Fourier da Função de autocorrelação de velocidades 108

5.5 Superposição de estruturas do complexo . . . . . . . . . . . . . . . . 110

5.6 Espectro calculado para o complexo $C_{4}(4 C P)^{+} I^{-} \ldots \ldots$. . . . . . . 111

5.7 Relação entre as energias de excitação calculadas em função da distância do iodeto ao carbono do anel. . . . . . . . . . . . . . . . . . . . . 111

5.8 Espectro simulado dos complexos a partir da BOMD de vácuo . . . . . 112

5.9 Comparação entre os comprimentos de ligação obtidos coma a MD e com a BOMD . . . . . . . . . . . . . . . . 118

5.10 Comparação entre os ângulos de ligação obtidos coma a MD e com a

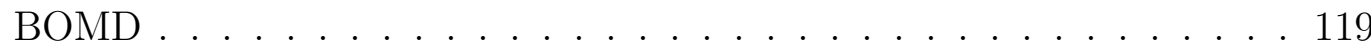

5.11 Comparação entre os ângulos diedros obtidos com a a MD e com a BOMD120

5.12 Evolução temporal de alguns parâmetros estruturais ao longo de dinâmica molecular clássica . . . . . . . . . . . . . . . . . . . 122

5.13 Representação da evolução estrutural do complexo ao longo da dinâmica 124

5.14 Evolução temporal do RMSD das estruturas do complexos em acetonitrila125

5.16 Espectro simulado parao pidínio isolado . . . . . . . . . . . . 127 
5.17 Espectro calculado separando as estruturas por similaridade . . . . . . 128

5.20 Dinâmica molecular clássica do complexo em água . . . . . . . . . . . . 132

A.1 Espectro vibracional infravermelho calculado . . . . . . . . . . . . . 178

A.2 Espectro de NMR calculado para o $C_{3} b i s(4 C P)^{2+} I^{-} \quad \ldots \ldots$. . . . . 181

B.1 Função de autocorrelação de velocidade para número crescente de pontos 187

B.2 A transformada de Fourier da função de autocorrelação de velocidades . 187

C.1 Representação estrutural da molécula de acetonitrila com a definição dos grupos de carga . . . . . . . . . . . . . . . . . . 190 


\section{Lista de Tabelas}

2.1 Escada de Jacó dos funcionais . . . . . . . . . . . . . . . . . . . . . . . . . . 29

4.1 Energias de interação dos complexos com brometo e iodeto e o erro de superposição de base . . . . . . . . . . . . . . . . . . 75

4.2 Comprimento de onda de excitação das 3 de excitações de mais baixa energia calculadas para o complexo formado pelo $C_{4}(4 C P)^{+}$com iodeto e brometo . . . . . . . . . . . . . . . . . . 81

4.3 Energias de excitação do complexo $C_{4}(4 C P)^{+} I^{-} \ldots \ldots$. . . . . . . . 83

4.4 Três excitações eletrônicas de mais baixa energia calculadas em acetonitrila (modelo contínuo) . . . . . . . . . . . . . 86

4.5 Comprimento de onda das excitações, calculadas em diferentes solventes (modelo contínuo $\ldots \ldots \ldots$. . . . . . . . . . . . . 88

4.6 Propriedades eletrônicas dos complexos . . . . . . . . . . . . . . . . . . 92

5.1 Modos vibracionais calculados na BOMD e calculados usando as geometrias otimizadas . . . . . . . . . . . . . . . 108

5.3 Parâmetros do campo de força . . . . . . . . . . . . . . . 116

5.4 Cargas parciais usadas no campo de força clássico . . . . . . . . . . 117 
A.1 Frequências vibracionais calculadas . . . . . . . . . . . . . . . . . . . . 182

D.1 Cargas parciais calculadas . . . . . . . . . . . . . . . . . 209 


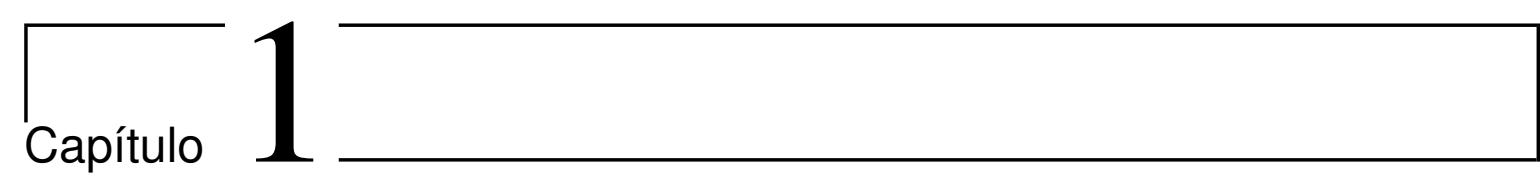

\section{Introdução}

O tema central deste trabalho é o estudo teórico dos complexos de transferência de carga formados por derivados piridínicos e iodeto. Neste capítulo apresentamos uma introdução a esses sistemas, bem como aos desafios experimentais e teóricos envolvidos no seu estudo. 


\subsection{Complexos moleculares}

Complexos moleculares são formados por associações entre moléculas com outras moléculas, átomos ou íons. Embora estáveis, são estabilizados por interações não covalentes mais fracas (1 a 2 ordens de magnitude) que as ligações covalentes responsáveis pelas ligações químicas [1]. Neste trabalho, foram estudados compostos conhecidos na literatura como complexos de transferência de carga (CTC, do inglês Charge-Transfer Complexes) ou ainda complexos EDA (EDA, do inglês Electron Donor-Acceptor) [2]. A formação desse tipo de complexo é caracterizada pelo aparecimento de uma banda adicional no espectro eletrônico de absorção.

Embora indícios da existência de CTC já tenham sido observados desde o século XIX [3], um passo crucial para a compreensão do fenômeno foi dado apenas em 1949, quando Benesi e Hildebrand publicaram um trabalho sobre o espectro eletrônico de absorção do iodo em diferentes solventes [4], mostrando o surgimento de uma banda adicional em $290 \mathrm{~nm}$ que não era observada em outros solventes e nem nas espécies individuais. O surgimento dessa banda era uma indicação clara da interação entre as duas moléculas e da possível formação de um complexo molecular.

As propriedades desses complexos foram finalmente compreendidas pouco tempo depois quando Robert Mulliken propôs um modelo teórico capaz de explicar essa associação [5]. Na teoria de Mulliken, o surgimento da banda de absorção é consequência da formação de um complexo molecular, estabilizado pela transferência de um elétron de uma espécie doadora (D) para uma espécie aceitadora (A), de acordo com a relação de equilíbrio [2]:

$$
D+A \stackrel{K_{C T C}}{\rightleftharpoons} D A
$$

onde $K_{C T C}$ é a constante de associação do complexo, e $D A$ descreve as espécies associadas.

Desde sua descoberta uma atenção considerável têm sido conferida aos CTC [6]. Eles são formados, por exemplo, em processos reativos no metabolismo de organismos vivos, de forma que as reações de transferência de carga que levam a formação desses complexos são de grande interesse [7]. No ramo farmacêutico, os CTC são usados na determinação espectrofotométrica de fármacos [8-11]. Na área de materiais, os conceitos relacionados aos CTC vêm ganhando destaque em áreas diversas como su- 
percondutores orgânicos, semicondutores, foto catálise [12-14] e no desenvolvimento de células solares orgânicas $[15,16]$. Eles têm sido usados também na criação de detectores de iodeto em eletrodos de íon seletivo $[17,18]$

\subsection{Constante de equilíbrio}

Em geral, toda a informação experimental acerca da interação entre o $D$ e o $A$ é inferida a partir da determinação da constante de equilíbrio, $K_{C T C}$ (ver Equação 1.1). Uma estimativa confiável dessa constante é, portanto, de suma importância.

A constante $K_{C T C}$ pode ser obtida a partir da medida do espectro eletrônico de absorção, desde que sejam assumidas algumas hipóteses. A primeira é delas é a estequiometria. Nos complexos formados por um único $D$ e um único $A$ (ou seja, estequiometria 1:1), o equilíbrio é estabelecido conforme a Equação (1.1), e a constante de equilíbrio químico é dada por

$$
K_{C T C}=\frac{[D A]}{[D][A]}
$$

onde $[D A],[A],[D]$ são as concentrações do CTC, da espécie aceitadora e da espécie doadora, respectivamente.

Sejam $[D]_{0}$ e $[A]_{0}$ as concentrações iniciais, antes que o equilíbrio seja estabelecido e, portanto antes que haja formação do complexo. Após o equilíbrio ser estabelecido teremos as seguintes concentrações: $[D A],[D]=[D]_{0}-[D A]$ e $[A]=[A]_{0}-[D A]$. Se $[D]_{0}>>[A]_{0}$ a concentração do doador no equilíbrio será essencialmente a mesma que antes do equilíbrio, ou seja, $[D] \simeq[D]_{0}$. Portanto,

$$
K_{C T C}=\frac{[D A]}{[D]_{0}\left([A]_{0}-[D A]\right)} .
$$

A concentração do complexo $D A$ pode ser relacionada com a absorbância da banda de transferência de carga usando a lei de Lambert-Beer,

$$
a=\varepsilon_{D A} b[D A],
$$


onde $a$ é a absorbância em um dado comprimento de onda, $\varepsilon_{D A}$ é a absorptividade molar nesse comprimento de onda, e $b$ é o comprimento do caminho ótico. Substituindo (1.4) em (1.3) e rearranjando os termos chega-se a expressão para a equação de BenesiHildebrand [4]:

$$
\frac{[A]_{0}}{a}=\frac{1}{\varepsilon_{D A}} b+\frac{1}{K_{C T C} \varepsilon_{D A} b}\left(\frac{1}{[D]_{0}}\right) .
$$

Fazendo as medidas da absorbância para diferentes concentrações do doador é possível obter a constante de equilíbrio a partir de um ajuste linear no gráfico de $[A]_{0} / a$ em função de $1 /[D]_{0}$.

\subsection{Complexos formados por iodeto e derivados pi- ridínicos}

Ainda na década de 1950, Kosower descobriu a capacidade de derivados piridínicos formarem complexos de transferência de carga com íons iodeto [19,20]. Ele estudava esses sistemas como um modelo, visando compreender o mecanismo de ação de coenzimas $\mathrm{NADP}^{+}$e $\mathrm{NAD}^{+}$, importantes no sistema metabólico e cujo sítio ativo é um anel piridínio. Kosower dedicou boa parte de sua pesquisa no estudo das propriedades desses complexos [21]. Uma das principais características observadas por ele era a alta sensibilidade da banda de transferência de carga ao meio no qual o complexo está inserido.

Por esta razão, estes complexos moleculares têm sido amplamente usados como sondas em solventes homogêneos [22], misturas binárias [23-29], membranas, miscelas e lipossomas [30-35]. Estudos da estabilidade, reatividade e caracterização dessas moléculas são, portanto, de grande importância para a compreensão de sistemas biológicos e vem sendo realizados nos últimos anos [22,36-43]. Recentemente os interesse em derivados piridínicos têm crescido também devido suas propriedades de líquidos iônicos [44-54].

Mas apesar da grande quantidade de resultados experimentais, sobretudo para o espectro de absorção destes complexos, não existe na literatura uma análise detalhada tanto da estrutura quanto das suas propriedades eletrônicas. 


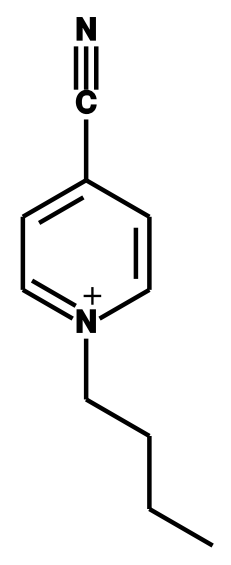

(a) $C_{4}(4 C P)^{+}$

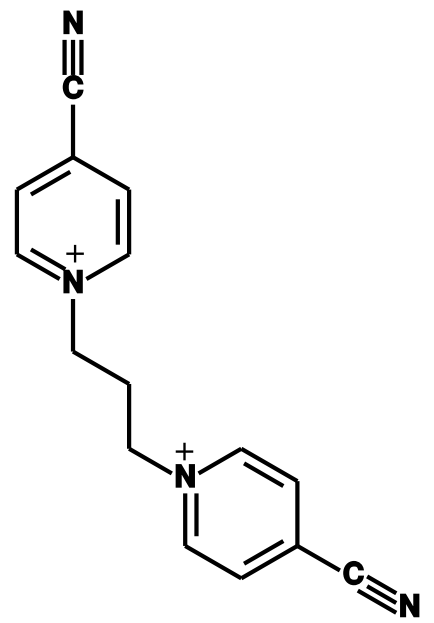

(b) $C_{3} b i s(4 C P)^{2+}$

Figura 1.1: Representação esquemática dos derivados piridínicos: (a) $C_{4}(4 C P)^{+} \mathrm{e}$ (b) $C_{3} b i s(4 C P)^{2+}$.

O objetivo deste trabalho é o estudo teórico dos complexos de transferência de carga formados por derivados da 4-cianopiridina com iodeto (mostrados na Figura 1.1), visando melhorar a compreensão das suas propriedades estruturais e eletrônicas, bem como aprofundar o entendimento tanto do comportamento quanto do espectro de absorção eletrônico apresentados por estes complexos em solução. Os derivados estudados foram o N-alquil-4-cianopiridínio - $C_{4}(4 C P)^{+}-$e o N,N'-alquildiil-bis(4-cianopiridínio) - $C_{3}$ bis $(4 C P)^{2+}$. O $C_{4}(4 C P)^{+}$(Figura 1.1a) é formado por apenas um anel piridínio e experimentalmente tem sido amplamente estudado desde a década de 1950 [21]. Já o $C_{3}$ bis $(4 C P)^{2+}$ (Figura 1.1b) é formado por dois anéis conectados por uma cadeia carbônica (derivado bispiridínico), tendo sido estudado recentemente por Hioka e colaboradores [34, 35, 55-58].

A adição de iodeto de potássio em uma solução contendo o $C_{4}(4 C P)^{+}$ou o $C_{3} b i s(4 C P)^{2+}$ leva ao aparecimento de uma banda adicional no espectro de absorção atribuída a formação de um CTC (Figura 1.2). Em acetonitrila, o máximo da banda está na região de $400 \mathrm{~nm}$ para os dois sistemas, embora o CTC formado pelo iodeto com o $C_{3}$ bis $(4 C P)^{2+}$ mostre uma banda mais intensa que o complexo com o $C_{4}(4 C P)^{+}$.

Conforme visto na seção anterior, uma das hipóteses fundamentais para obter a constante usando a Equação (1.5) é a estequiometria do complexo. Curiosamente os 


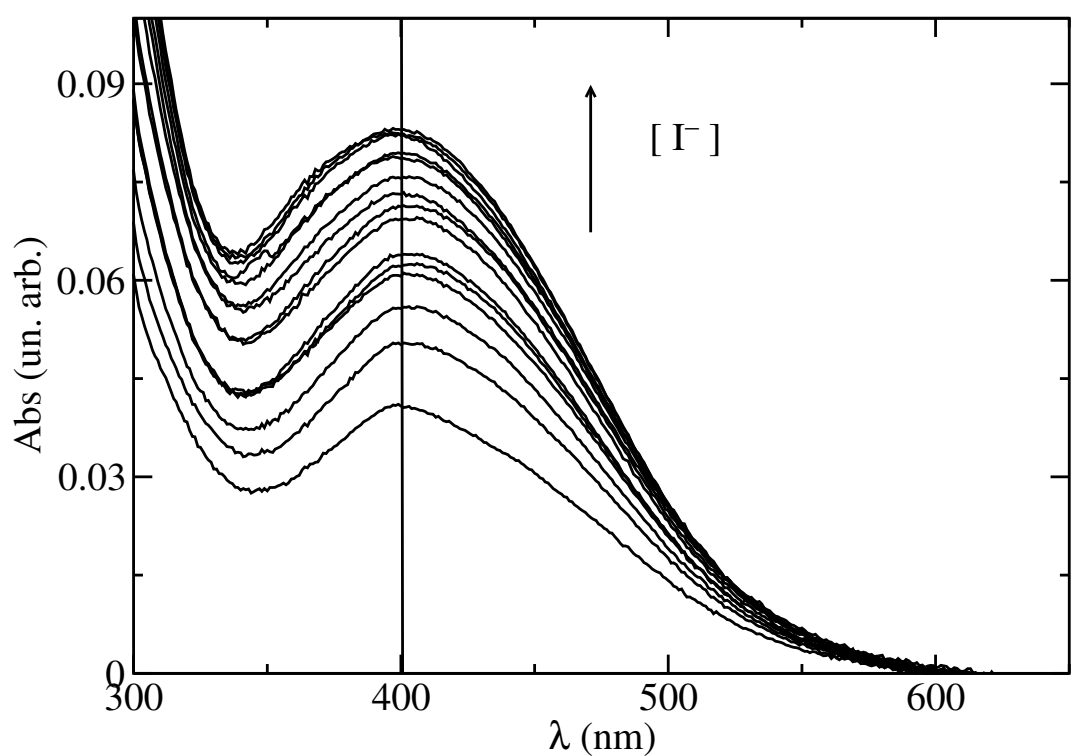

Figura 1.2: Espectro de absorção experimental medido a $30^{\circ} \mathrm{C}$ em acetonitrila para o derivado $C_{3}$ bis $(4 C P)^{2+}\left(5.0 \times 10^{-5}\right.$ mol. $\left.\mathrm{L}^{-1}\right)$. Observa-se o surgimento e o aumento da intensidade da banda com o aumento da concentração de $I^{-}$(de 0 a 0.072 mol.L $\left.\mathrm{L}^{-1}\right)$, resultado da adição de iodeto de potássio na solução. Figura retirada da referência [35].

resultados experimentais indicam uma relação estequiométrica equimolar (1:1) tanto para o complexo de $I^{-}$com o $C_{4}(4 C P)^{+}$quanto para o complexo com o $C_{3} b i s(4 C P)^{2+}$. Estes resultados foram obtidos usando um método padrão, conhecido como método das variações contínuas ou método de Job [59-62]. Neste método, a razão estequiométrica é determinada pelo ponto de máximo no gráfico da absorbância em função da fração molar do doador, conforme mostrado na Figura 1.3 (retirado da referência [35]).

No caso do $C_{3} b i s(4 C P)^{2+}$, que é composto por duas unidades de piridínio, seria razoável esperar a associação de um $I^{-}$a cada anel aromático, o que resultaria em uma estequiometria 2:1. A explicação dada pelos experimentais para a estequiometria 1:1 seria o surgimento de uma conformação do tipo sanduíche [35], onde um $I^{-}$estaria inserido entre os dois anéis aromáticos (ver Figura 1.4). Embora seja razoável, nenhuma medida adicional foi apresentada para corroborar com essa hipótese.

Vale ressaltar que uma conformação semelhante a essa já havia sido proposta experimentalmente para explicar a reação do $C_{3} b i s(4 C P)^{2+}$ com os ânions hidróxido $\left(\mathrm{OH}^{-}\right)$em meio alcalino [34,57], onde se observa a formação de um produto de reação adicional em relação ao caso da reação $C_{4}(4 C P)^{+}+O H^{-}$. A formação de um com- 
plexo sanduíche entre o $C_{3} b i s(4 C P)^{2+}$ foi usada para explicar a formação do composto adicional [34].

Nas estimativa da constante de equilíbrio assume-se ainda que a banda em 400 nm é formada apenas pela excitação do CTC com iodeto. A banda de menor comprimento de onda que aparece em cerca de $300 \mathrm{~nm}$ (ver Figura 1.2) é atribuída as demais espécies em solução, principalmente pela absorção interna no anel dos derivados piridínicos. É importante ressaltar que tanto o $C_{4}(4 C P)^{+}$quanto o $C_{3} b i s(4 C P)^{2+}$ são sintetizados tendo como contra-íon o $\mathrm{Br}^{-}$, e a formação de um CTC com este contra-íon em solução não pode ser descartada. Mas não é esperado que eles tenham alguma influência na banda de $400 \mathrm{~nm}$, uma vez que dados experimentais obtidos para complexos similares mostram que a banda de complexos com $\mathrm{Br}^{-}$estão em regiões de menor comprimento de onda em relação aos mesmos complexos formados com $\mathrm{I}^{-}[63,64]$. Experimentalmente observa-se ainda o aumento da absorbância na região de $300 \mathrm{~nm}$ e a diminuição da banda em $400 \mathrm{~nm}$ com a adição de $B r^{-}$na solução [35], o que indica que a banda de um eventual complexo com brometo estaria na mesma região dos derivados piridínicos.

Como a complexidade dos fenômenos em solução tornam bastante difícil a tarefa de validar as hipóteses experimentais, o estudo teórico dos complexos pode contribuir bastante para a compreensão da propriedades dos complexos em solução. O uso de ferramentas teóricas e computacionais, sobretudo quando combinadas com as técnicas experimentais, tem contribuído de forma essencial para a compreensão dos fenômenos em ambientes complexos. 


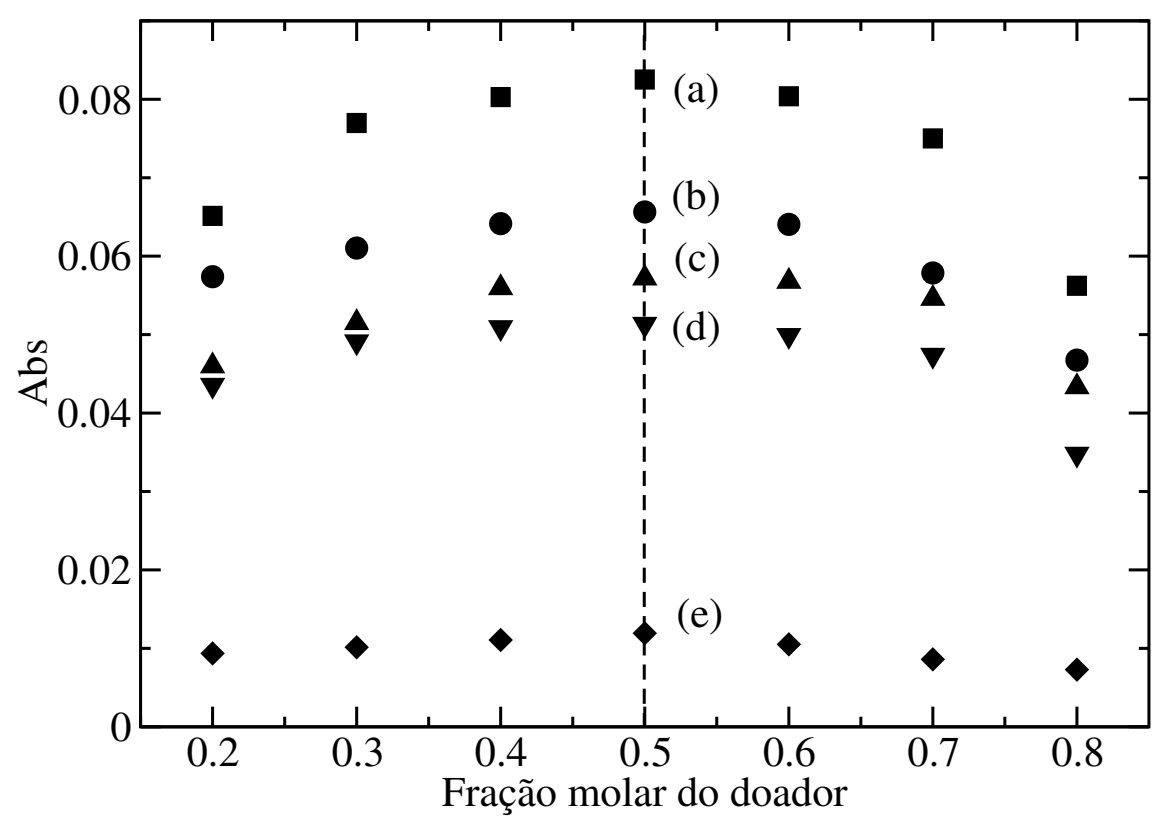

Figura 1.3: Ajuste usado na determinação da estequiometria dos CTC formados em acetonitrila pelo I ${ }^{-}$com os derivados: (a) $C_{3} b i s(4 C P)^{2+}$, (b) $C_{4} b i s(4 C P)^{2+}$, (c) $C_{4} b i s(4 C P)^{2+}$ (c) $C_{6} b i s(4 C P)^{2+}$ (e) $C_{6}(4 C P)^{+}$. Figura retirada da referência [35].
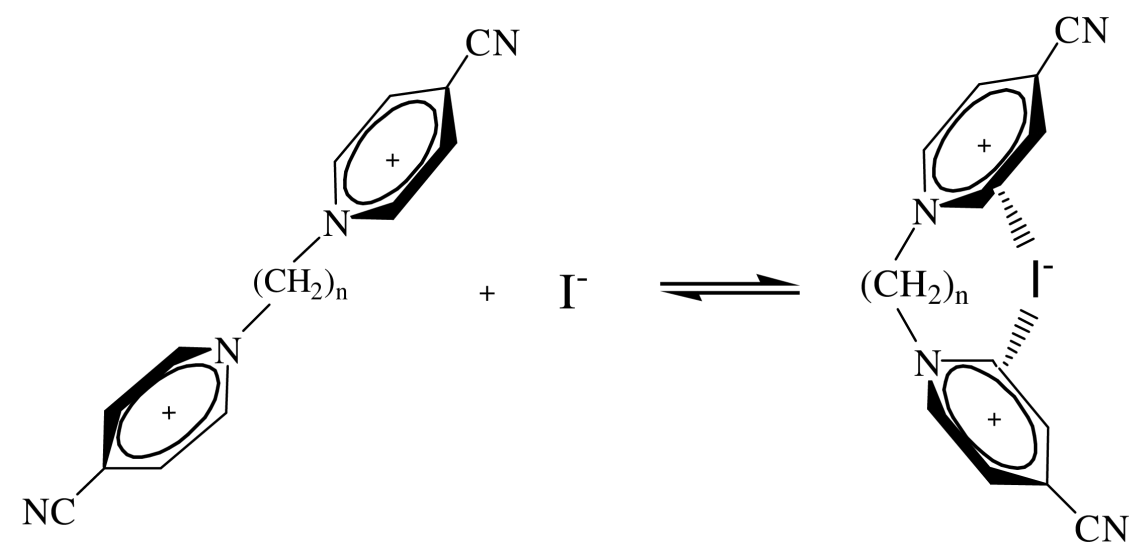

Figura 1.4: Representação esquemática do equilíbrio químico proposto experimentalmente para a associação entre $I^{-}$e o $C_{3} b i s(4 C P)^{2+}$. Figura retirada da referência [35]. 


\subsection{Estudo teórico de complexos de transferência de carga}

Do ponto de vista teórico, o estudo de CTC é bastante desafiador. A maior parte dos complexos são compostos por moléculas aromáticas neutras e estabilizados por meio de interações não covalentes como forças de van der Waals. O emprego de métodos pós-Hartree-Fock (pós-HF) com elevado nível de correlação eletrônica, capazes de uma descrição acurada dessas interações, são limitados a sistemas moleculares com um número reduzido de elétrons, dado o elevado custo computacional. O mesmo pode ser dito para as energias de excitação. O estado da arte no cálculo das excitações eletrônicas são métodos multiconfiguracionais, como CASPT2 [65,66]. O uso desses métodos é limitado principalmente pelo alto custo computacional. Sua implementação ainda é restrita a alguns programas, embora venham se difundido cada vez mais.

A teoria do funcional da densidade (DFT, do inglês "Density Functional Theory") se estabeleceu nos últimos anos como uma alternativa aos métodos ab initio pósHF [67]. Com uma abordagem conceitualmente mais simples, usando como ingrediente básico a densidade eletrônica ao invés da função de onda, a DFT consegue, em muitos casos, resultados comparáveis aos dos métodos pós-HF a um custo computacional muito menor. Por essa razão, a DFT tornou-se a teoria mais usada atualmente no estudo das propriedades eletrônicas de átomos, moléculas e sólidos. Pela criação da DFT, Walter Kohn recebeu o prêmio Nobel de química no ano de 1998 [68].

O emprego da DFT no estudo teórico de CTC tem se mostrado um grande desafio [69], tanto no cálculo da energia de interação [70], quanto das energias de excitação dos complexos [71] (nesse último caso o cálculo é realizado usando a teoria do funcional da densidade dependente do tempo). A maior parte dos funcionais tradicionalmente usados em estudos teóricos comete erros enormes na descrição das excitações dos CTC [72]. Por outro lado, esse tipo de sistema impulsionou o desenvolvimento de novos funcionais, capazes de lidar com o problema da transferência de carga. Atualmente já é possível descrever excitações de transferência de carga com bastante precisão [73-76].

Outro desafio no estudo dos CTC estudados neste trabalho é que, devido sua grande sensibilidade ao meio, o efeito do solvente não pode ser desprezado. A maior 
parte dos trabalhos teóricos, entretanto, não inclui o efeito do solvente [71,77]. Os trabalhos que o fazem, empregam apenas modelos contínuos de solvatação [8]. Não existe na literatura nenhum estudo sistemático sobre a inclusão do efeito do solvente de forma explícita e discreta sobre complexos de transferência de carga.

\subsection{Estudo teórico de moléculas em solução}

\subsubsection{Abordagem supermolecular e modelos contínuos}

A complexidade dos fenômeno físico-químicos em solução tornou necessário o desenvolvimento de uma ampla variedade de modelos teóricos e técnicas computacionais para o estudo de moléculas em solução.

A idéia mais direta de se obter a propriedade de um sistema em solução seria usar uma abordagem supermolecular, que consiste na inclusão de moléculas de solvente ao redor do soluto. Dessa forma o cálculo das propriedades são realizados no superaglomerado soluto-solvente em uma configuração de mínina energia. Não só o alto custo computacional dos métodos de mecânica quântica (QM) inviabilizam a aplicação desse tipo de abordagem em larga escala, como também as fracas interações intermoleculares tornam a convergência dos procedimentos de minimização de energia um processo bastante delicado.

Além disso, a grande mobilidade das moléculas em solução inviabiliza, na maior parte dos casos, o uso de uma aproximação única para a geometria do sistema. Em uma representação mais realista uma propriedade deve ser calculada como média sobre várias configurações distintas, escolhidas de forma a amostrar o ensemble estatístico a uma dada condição termodinâmica de equilíbrio.

Outra abordagem amplamente utilizada são modelos contínuos de solvatação, onde o meio solvente é descrito como um meio contínuo, homogêneo e isotrópico, caracterizado principalmente por sua constante dielétrica $\varepsilon$. Desenvolvidos inicialmente ainda na década de 1930 por Born [78], Osager [79] e Kikwood [80], esses modelos foram aprimorados e adaptados aos problemas de modelagem computacional na década de 1970 ao mesmo tempo por Tapia e Goscinski [81] e por Rivail e Rinaldi [82], com a formulação do campo de reação autoconsistente (SCRF, do inglês "self consistent 
reaction-field").

Nestes modelos o soluto é colocado em uma cavidade imersa no meio dielétrico que representa o solvente. A distribuição inicial de cargas do soluto polariza o meio dielétrico, induzindo cargas na superfície da cavidade. Essas cargas induzidas, por sua vez, geram um campo elétrico sobre o soluto (o campo de reação). Um procedimento autoconsistente de polarização do soluto é então adotado: novas cargas do soluto são calculadas, agora na presença do campo. As nova distribuição de carga do soluto gera novas cargas sobre a superfície da cavidade e um novo campo de reação. O procedimento é repetido até a convergência.

Um dos modelos contínuos mais eficientes e de maior sucesso é o modelo contínuo polarizável (PCM, do inglês "Polarizable Continuum Model") proposto por Tomasi e colaboradores em 1981 [83] e desenvolvido até hoje [84-86].

A interação da molécula com o campo de reação é então adicionada no hamiltoniano quântico como uma perturbação externa. A grande vantagem dos modelos contínuos é a possibilidade de incluir a interação soluto-solvente no problema de mecânica quântica sem aumentar o número de elétrons, o que não aumenta o custo computacional de forma significativa. Em geral, os modelos contínuos também conseguem obter um tratamento bastante acurado da interação eletrostática entre o soluto e o solvente.

A grande desvantagem é que propriedades moleculares que exigem um descrição mais detalhada da interação soluto-solvente não podem ser obtidas por esse tipo de modelo. Interações específicas, sobretudo na primeira camada de solvatação, como por exemplo ligações de hidrogênio, interação de dispersão e transferência de carga são desafiadores para esse tipo de modelo. Nesses casos, ao menos ao redor do soluto algumas moléculas explícitas de solvente devem ser adicionadas ao problema.

Ainda assim, persiste o problema de quantas moléculas de solvente devem ser incluídas no cálculo, e onde posicioná-las. Essa questão é respondida de forma natural nos chamados métodos de simulação computacional, onde as configurações acessíveis aos sistemas na fase condensada são geradas de forma eficiente e sistemática [87]. Dentre estes metódos, conhecidos sob o nome genérico de Mecânica Molecular (MM), destacam-se o método Monte Carlo a Dinâmica Molecular ou MD (do inglês "Molecular Dynamics"). 


\subsubsection{Dinâmica molecular}

O método dinâmica molecular reúne um amplo número de técnicas que podem ser classificadas em dois grandes grupos: a dinâmica molecular clássica e a dinâmica molecular por primeiros princípios ou dinâmica molecular ab initio (AIMD). Esta utiliza um potencial de interação ab initio oriundo de uma solução (ou, pra ser mais preciso, de uma solução aproximada) para a equação de Schrödinger enquanto que aquela utiliza um potencial efetivo para modelar as interações.

A dinâmica molecular clássica constitui uma ferramenta poderosa e bem estabelecida usada no estudo de sistemas na fase condensada [87]. A amplitude, a diversidade e o nível de sofisticação desta técnica é amplamente documentada na literatura [88-93].

A questão central em qualquer dinâmica molecular reside na descrição das interações interatômicas. Na dinâmica molecular clássica o potencial de interação é separado em contribuições de um corpo, dois corpos, e assim por diante, em contribuições de curto e longo alcance, etc., e cada termo é representado por uma forma funcional adequada. Após décadas de intenso desenvolvimento, formas funcionais bastante sofisticadas foram concebidas a fim de representar os aspectos nem sempre triviais dessas interações.

Os reinos já tradicionais da dinâmica molecular e dos métodos de estrutura eletrônica foram estendidos com o advento das técnicas de dinâmica molecular por primeiros princípios. Convém ressaltar que, apesar da terminologia ab initio, a AIMD também é uma teoria clássica, no sentido de que os núcleos se movem de acordo com equações clássicas de movimento. Obviamente o potencial de origem quântica usado na AIMD torna a técnica extremante poderosa, sendo capaz de incluir naturalmente fenômenos descritos com dificuldade pelos potenciais de interação clássicos, como polarização, transferência de carga, etc. A inclusão explícita dos graus de liberdade eletrônicos permite também monitorar a evolução temporal de propriedades eletrônicas e descrever inclusive reações químicas, tarefas impossíveis de serem realizadas usando apenas a dinâmica molecular clássica.

As bases teóricas para a dinâmica ab initio foram estabelecidas ainda na década de 1920 com os trabalhos de Dirac e Ehrenfest [94]. Mas o grande avanço no que se refere à aplicação do método a sistemas em fase condensada ocorreu apenas em 1985 com 
o surgimento do método proposto por Robert Car e Michele Parrinello (CPMD) [95]. Usando uma técnica de Lagrangiana estendida, Car e Parrinello conseguiram reduzir o custo computacional dos cálculos de estrutura eletrônica ao longo da trajetória, criando assim uma rota para a aplicação da AIMD a sistemas em fase condensada e que serviu base para futuros desenvolvimentos no campo. A CPMD pode ser considerada uma aproximação para a chamada dinâmica molecular de Born-Oppenheimer (BOMD) [96]. A diferença crucial é que na BOMD a estrutura eletrônica é convergida a cada passo, ou seja, a dinâmica é realizada sobre a superfície de energia potencial de Born-Oppenheimer (daí a origem do nome dado ao método), o que torna o método conceitualmente mais simples do que a CPMD.

Infelizmente a aplicação, os cálculos da AIMD ainda são bastante dispendiosos pois são realizados um cálculo de estrutura eletrônica e um cálculo de gradiente a cada passo. Este passo é geralmente de 0.1-0.5 fs para a BOMD e cerca de 10 vezes menor para a CPMD. Atualmente a aplicação dessas técnicas é limitada a sistemas da ordem de 100 átomos e a trajetórias de no máximo 100 ps.

\subsubsection{A combinação entre mecânica quântica e mecânica mo- lecular}

O surgimento dos métodos QM/MM [97-100] proporcionou uma grande evolução no estudo de propriedades eletrônicas e da reatividade de sistema moleculares e biomoleculares em ambientes complexos. Por essa razão, Martin Karplus, Michael Levitt e Arieh Warshel, pioneiros nesse tipo de abordagem [97,101], receberam o prêmio Nobel de Química de 2013 [102-104].

A idéia dos métodos híbridos é combinar as duas metodologias QM e MM, de tal forma que a região de maior interesse do sistema é calculada usando mecânica quântica e a vizinhança é tratada usando a mecânica molecular. A grande vantagem dos métodos híbridos em relação aos modelos contínuos é a possibilidade de tratar o sistema como um todo usando moléculas explícitas. Além disso o custo computacional é bastante reduzido em relação à AIMD, permitindo o tratamento de sistemas mais realistas na fase condensada.

Sob o nome genérico de QM/MM existem atualmente um grande número de 
técnicas, dentre as quais destacam-se a QM/MM convencional e a QM/MM sequencial. $\mathrm{Na}$ QM/MM convencional o Hamiltoniano do sistema é escrito na forma

$$
H=H_{Q M}+H_{M M}+H_{Q M / M M}
$$

onde $H_{Q M}$ é o Hamiltoniano quântico e $H_{M M}$ a Hamiltoniana clássica. O termo $H_{Q M / M M}$ é responsável pelo acoplamento entre a parte quântica e a parte clássica.

Uma das questões mais delicadas na QM/MM convencional reside na escolha adequada da partição quântica (QM) do sistema. Especial atenção deve ser conferida aos termos de acoplamento na fronteira entre a parte quântica e a parte clássica, sobretudo no caso de haver quebra de ligações [99,105].

Ainda que tenha se tornado uma ferramenta importante no estudo de sistemas biológicos na fase condensada $[99,106]$, o custo computacional de uma dinâmica QM/MM permanece elevado, uma vez que ainda são necessários realizar um número grande de cálculos de estrutura eletrônica (tipicamente centenas de milhares), ainda que em uma parte reduzida do sistema.

Já o método híbrido QM/MM sequencial é baseado na separação dos cálculos MM e QM em duas etapas sequenciais independentes. Na primeira é realizada a simulação computacional do sistema, onde são geradas as configurações de equilíbrio acessíveis ao sistema a uma dada condição termodinâmica. Na segunda etapa são realizados os cálculos QM das propriedades nas configurações de equilíbrio obtidas na primeira etapa. A grande vantagem da separação consiste na possibilidade de reduzir drasticamente o número de cálculos QM, o que também reduz consideravelmente o custo computacional.

O uso combinado de simulação computacional e cálculos quânticos já havia sido explorado em trabalhos como os de Blair e co-autores [107, 108] e os de Zeng e coautores [109-112]. As idéias foram finalmente sistematizadas de uma forma eficiente no chamado método S-QM/MM (Sequential Quantum Mechanics/Molecular Mechanics) proposto pouco tempo depois por Canuto e Coutinho [113-116].

Na abordagem S-QM/MM, usa-se um eficiente método de amostragem para obter resultados estatisticamente convergidos com um reduzido número de cálculos quânticos. A primeira etapa consiste de uma simulação clássica para gerar a estrutura do 
líquido (configurações de equilíbrio). Em geral, nesta etapa é usado o método de Monte Carlo (S-MC/QM), embora não haja impedimentos para o uso da dinâmica molecular. Assim são geradas as configurações do líquido.

Duas importantes análises são realizadas nessas configurações, visando reduzir o custo computacional nos cálculos quânticos posteriores. A primeira análise está relacionada a correlação estatística entre estas configurações, que permite escolher conveniente um número reduzido de configurações para serem submetidas aos cálculos quânticos (tipicamente são menos de 100). A segunda análise é o particionamento do sistema que será tratado explicitamente no cálculo quântico (em geral são tratados explicitamente o soluto e algumas moléculas de solvente, que podem ser as ligações de hidrogênio, a primeira camada de solvatação, etc.). As propriedades são então obtidas como médias simples dos cálculos QM realizados nestas configurações.

Assim, na segunda etapa (QM) os cálculos quânticos são realizados em configurações descorrelacionadas, o que possibilita obter médias convergidas para as propriedades de interesse com um número reduzido de configurações (tipicamente são da ordem de um centena) $[114,117,118]$.

A S-QM/MM tem uma série de vantagens em relação ao QM/MM convencional. O custo computacional é muito menor, já que o número de cálculos quânticos são reduzidos para cerca de uma centena. Além disto, eles são realizados em uma simulação já convergida. Também vale ressaltar que uma mesma simulação pode ser usada para o cálculo de várias propriedades eletrônicas. Por último, existe uma liberdade maior na escolha da partição do sistema que será submetida ao cálculo quântico.

Evidentemente, esta metodologia tem suas desvantagens. A principal está relacionada ao desacoplamento entre as partes quântica e clássica, que não permite que ela seja usada em sistemas onde este acoplamento é relevante. Ainda assim, esta abordagem tem sido usada com êxito no estudo de diversas propriedades, como estudos de espectroscopia de absorção e emissão, parâmetros de NMR, ligações de hidrogênio, solvatação diferencial, etc. Essa abordagem tem sido usada com êxito no estudo de diversas propriedades, como estudos de espectroscopia de absorção [117,119-126] e de emissão [127,128], ligações de hidrogênio [118,129,130], parâmetros de NMR [131-135], solvatação e propriedades termodinâmicas [136-141], etc. 


\subsection{Organização da tese}

A este capítulo introdutório somam-se mais cinco capítulos, de forma que a presente tese é composta ao todo de seis capítulos.

No capítulo 2 são apresentados os principais métodos teóricos usados no estudo da estrutura eletrônica dos complexos. Uma vez definido o problema eletrônico a partir da aproximação de Born-Oppenheimer sobre a equação de Shrödinger do sistema molecular, são apresentados os fundamentos da teoria do funcional da densidade na formulação de Kohn-Sham e os funcionais de troca e correlação. Em seguida apresentase a teoria do funcional da densidade dependente do tempo que, na aproximação de resposta linear, permite o cálculo das energias de excitação do sistema.

O método dinâmica molecular é discutido no capítulo 3. O início do capítulo é dedicado a dinâmica molecular de Born-Oppenheimer, bem como alguns detalhes da implementação usada neste trabalho. Em seguida a dinâmica molecular clássica (usando campos de força) é apresentada. Alguns procedimentos comuns aos dois métodos são discutidos no final do capítulo, como a integração das equações de movimento, termostatos, etc. e alguns procedimentos de análise.

A estrutura eletrônica para os CTC foi estudada no capítulo 4, usando as geometrias otimizadas. Inicialmente são apresentados os resultados para o complexo $C_{4}(4 C P)^{+}$com brometo e iodeto. Em seguida, para o complexo $C_{3} b i s(4 C P)^{2+}$ com iodeto. Os resultados também foram estendidos para os complexos em solução usando o modelo contínuo polarizável.

No capítulo 5 são apresentados resultados da dinâmica molecular dos complexos. Incialmente são apresentados os resultados obtidos usando a dinâmica de BornOppenheimer. Após a análise desta dinâmica, os resultados foram usados para refinar alguns parâmetros do campo de força clássico. Assim foi possível realizar a dinâmica molecular dos complexos em solução, a partir do qual foi possível, usando o procedimento sequencial, obter espectro de absorção dos complexos em solução.

As considerações finais são apresentadas no capítulo 6 . 


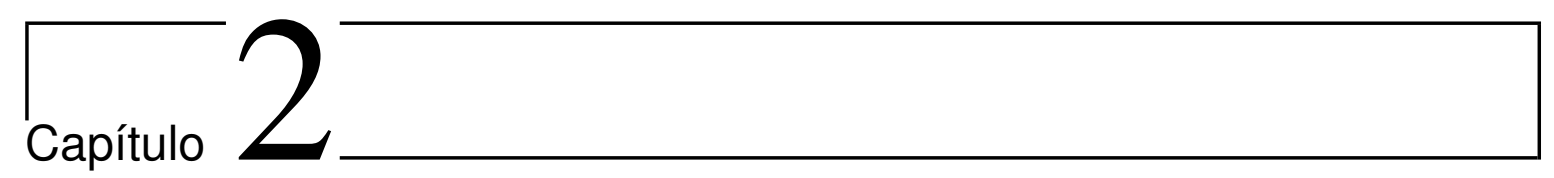

\section{Fundamentos da DFT e da TDDFT}

Este capitulo apresenta uma introdução aos principais métodos teóricos empregados ao longo do trabalho no estudo das propriedades eletrônicas dos CTC: a teoria do funcional da densidade (DFT) e a teoria do funcional da densidade dependente do tempo (TDDFT). 


\subsection{Introdução}

Este capítulo apresenta uma introdução aos principais métodos teóricos empregados ao longo do trabalho no estudo das propriedades eletrônicas dos CTC: a teoria do funcional da densidade (DFT) e a teoria do funcional da densidade dependente do tempo (TDDFT). Serão apresentados apenas os conceitos básicos e o quadro formal de cada teoria, uma vez que já existe uma literatura bastante ampla sobre o assunto. Aqueles que desejarem um tratamento mais aprofundado são encorajados a consultálos.

Em relação a DFT, existem diversos livros-texto que abordam o assunto, tanto do ponto de vista introdutório $[142,143]$ quanto em tratamentos mais avançados $[144,145]$. Boa parte do conteúdo desse capítulo é baseado nas introduções feitas por Capelle [146] e por Burke e Wagner [147].

Já a TDDFT é abordada de maneira bastante aprofundada em dois excelentes livros-texto $[148,149]$, além dos artigos de revisão [150-152]. Seguimos de perto o tratamento feito por Ullrich na referência [153].

A DFT é uma teoria do estado fundamental: ela é usada para encontrar o estado fundamental de um sistema quântico e calcular as correspondentes quantidades de interesse, tais como a energia, dipolo, comprimentos de ligação, etc. Os primórdios da DFT datam de 1964/65, quando os famosos trabalhos de Hohenberg e Kohn [154] e Kohn e Sham [155] foram publicados. Atualmente a DFT é o método dominante para cálculos de estrutura eletrônica na Física, Química, e muitas outras áreas (ver referência [67] para um cenário atual acerca da DFT).

Embora de origem muito mais recente [156], a TDDFT alcançou um status semelhante no que se refere ao cálculo de excitações eletrônicas. Formalmente, no entanto, a TDDFT é uma abordagem teórica para a equação de movimento quântica, sendo portanto adequada para descrever sistemas quânticos não estacionários.

\subsection{O problema eletrônico}

A equação de Schrödinger não relativística independente do tempo para um sistema molecular formado por $M$ núcleos descritos pelas coordenadas $\mathbf{R} \equiv\left\{\mathbf{R}_{1}, \ldots, \mathbf{R}_{M}\right\}$ 
e $\mathrm{N}$ elétrons descritos pelas coordenadas $\mathbf{r} \equiv\left\{\mathbf{r}_{1}, \ldots, \mathbf{r}_{N}\right\}$ e variáveis de $\operatorname{spin} \sigma \equiv$ $\left\{\sigma_{1}, \ldots, \sigma_{N}\right\}$, é dada por:

$$
\mathcal{H} \Psi(\mathbf{x} ; \mathbf{R})=E \Psi(\mathbf{x} ; \mathbf{R})
$$

onde $\mathbf{x}=(\mathbf{r}, \sigma)$ representam conjuntamente as variáveis espaciais e de spin. O operador Hamiltoniano total do sistema, em unidades atômicas $(e=\hbar=m=1)$, na ausência de campos externos, tem a forma

$$
\mathcal{H}=\underbrace{-\sum_{i=1}^{N} \frac{1}{2} \nabla_{i}^{2}}_{T_{e}}-\underbrace{-\sum_{A=1}^{M} \frac{1}{2 M_{A}} \nabla_{A}^{2}}_{T_{N}}-\underbrace{-\sum_{i=1}^{N} \sum_{A=1}^{M} \frac{Z_{A}}{r_{i A}}}_{V_{e N}}+\underbrace{\sum_{i=1}^{N} \sum_{j>i}^{N} \frac{1}{r_{i j}}}_{V_{e}} \underbrace{\sum_{A=1}^{M} \sum_{B>A}^{M} \frac{Z_{A} Z_{B}}{R_{A B}}}_{V_{N N}},
$$

onde $M_{A}$ é a massa nuclear, $r_{i j}=\left|\mathbf{r}_{\mathbf{i}}-\mathbf{r}_{\mathbf{j}}\right|, R_{i A}=\left|\mathbf{r}_{\mathbf{i}}-\mathbf{R}_{\mathbf{A}}\right|$ e $R_{A B}=\left|\mathbf{R}_{\mathbf{A}}-\mathbf{R}_{\mathbf{B}}\right|$ são posições relativas entre elétron-elétron, elétron-núcleo e núcleo-núcleo. $\nabla_{i}^{2}$ e $\nabla_{A}^{2}$ envolvem diferenciação em relação as coordenadas do i-ésimo elétron e do A-ésimo núcleo.

O ponto de partida no estudo de um sistema molecular consiste na separação dos movimentos nuclear e eletrônico, usando a chamada aproximação de BornOppenheimer [157]. O fato é que os prótons e nêutrons que compõem o núcleo atômico são muito (1836 vezes) mais pesados do que os elétrons. Por essa razão, é razoável esperar que os núcleos se movam muito mais lentamente do que a nuvem eletrônica. Por outro lado os elétrons devem se adaptar quase instantaneamente ao movimento nuclear.

Sendo assim, busca-se uma solução para a equação (2.1) na forma de um produto

$$
\Psi(\mathbf{r}, \mathbf{R})=\Psi_{e}(\mathbf{r} ; \mathbf{R}) \Phi_{N}(\mathbf{R})
$$

onde $\Phi_{N}(\mathbf{R})$ é a função de onda nuclear e $\Psi_{e}(\mathbf{x} ; \mathbf{R})$ é a função de onda eletrônica, que depende das coordenadas eletrônicas e parametricamente das coordenadas nucleares. Assumindo então que o operador $T_{N}$ não atua sobre as função de onda eletrônica é possível separar o problema em duas equações, uma para os elétrons:

$$
\left[\hat{T}_{e}+\hat{V}_{e N}+\hat{V}_{e}\right] \Psi(\mathbf{x}, \mathbf{R})=E(\mathbf{R}) \Psi(\mathbf{x}, \mathbf{R})
$$


e outra para os núcleos:

$$
\left[\hat{T}_{N}+V_{N N}(\mathbf{R})+E(\mathbf{R})\right] \Phi_{N}(\mathbf{R})=\mathcal{E} \Phi_{N}(\mathbf{R}) .
$$

Observando a equação eletrônica (2.4), nota-se que os níveis de energia eletrônicos, $E(\mathbf{R})$, dependem da configuração nuclear (essa configuração dá a noção de geometria molecular), de forma que para a solução do problema eletrônico, é preciso especificar uma configuração nuclear fixa (a configuração que minimiza a energia, por exemplo). A energia total $V_{N N}(\mathbf{R})+E(\mathbf{R})$, por outro lado, tem a interpretação de energia potencial na equação (2.5), ou seja, esse termo é responsável pela criação da superfície de energia potencial no qual os núcleos se movem.

A solução do problema nuclear depende, portanto, da solução do problema eletrônico, ou de uma boa aproximação para a energia total. Uma aproximação clássica para a equação de movimento nuclear leva diretamente a dinâmica molecular clássica, como será abordado em detalhes no próximo capítulo. Por hora, o problema consiste em encontrar uma solução para o problema eletrônico (2.4).

Tradicionalmente, aproximações para a solução problema eletrônico (2.4) são obtidas usando os chamados métodos baseados em função de onda, dentre os quais os mais usados são os métodos de Hartree-Fock (HF) e pós-HF. No método de HF, o potencial de interação entre os elétrons $\hat{V}_{e}$ é substituído por um potencial efetivo $\hat{V}_{H F}$ que representa um campo externo gerado pelo potencial nuclear e pelo potencial médio dos outros $N-1$ elétrons [158]. Entretanto, as correlações eletrônicas não podem ser descritas por potenciais de um corpo como o de HF e espera-se apenas que propriedades que não dependam de forma essencial dessas correlações possam ser bem descritas por esse potencial. O método de HF têm sua importância também, pois as soluções servem de referência para métodos mais acurados (por isso chamados de pós-HF) usados na busca da correlação perdida, como por exemplo, para a Teoria de Perturbação de Möller Plesset (MP), Configuração de Interação (CI) e CoupledCluster (CC) [158]. Embora sejam capazes de produzir resultados bastante acurados, os métodos pós-HF possuem um custo computacional que cresce muito rapidamente com o tamanho do sistema.

Uma abordagem conceitualmente diferente para a solução do problema eletrônico 
é a DFT [68], onde a densidade eletrônica é usada como variável fundamental. Essa teoria tornou-se uma alternativa poderosa aos métodos pós-HF, pois é capaz de produzir, em muitos casos, resultados semelhantes a um custo computacional comparável ao método HF.

\subsection{A teoria do funcional da densidade}

Algumas simplificações serão usadas daqui para frente:

(1) Considere o problema eletrônico (2.4) escrito na seguinte forma,

$$
\left[\hat{T}+\hat{V}_{e}+\hat{V}_{e x t}\right] \Psi(\mathbf{r})=E \Psi(\mathbf{r})
$$

onde, ao invés do potencial $\hat{V}_{e N}$, foi usado um potencial externo mais geral, da forma

$$
\hat{V}_{e x t}=-\sum_{i=1}^{N} v\left(\mathbf{r}_{i}\right)
$$

(2) Por simplicidade, as variáveis de spin não serão incluídas. Em outras palavras, $\Psi_{j}\left(\mathbf{r}_{1}, \ldots, \mathbf{r}_{N}\right)$ descrevem elétrons sem spin. No caso de se incluir as variáveis de spin, a função de onda é escrita como na seção anterior, ou seja, $\Psi\left(\mathbf{x}_{1}, \ldots, \mathbf{x}_{N}\right)$, onde $\mathbf{x}_{i}=\left(\mathbf{r}_{i}, \sigma_{i}\right)$ denotam as coordenadas espaciais e de spin do $i$-ésimo elétron.

A abordagem usual da Mecânica Quântica consiste em, uma vez explicitado a forma funcional para o potencial externo $v\left(\mathbf{r}_{i}\right)$, buscar uma função de onda $\Psi(\mathbf{r})$ que é solução (ou pelo menos, uma boa aproximação) para a equação se Schrödinger (2.6). Uma vez conhecida $\Psi(\mathbf{r})$, qualquer observável pode ser calculado. Um desses observáveis é a densidade eletrônica:

$$
\rho(\mathbf{r})=N \underbrace{\iint \cdots \int}_{N-1}\left|\Psi\left(\mathbf{r}, \mathbf{r}_{2}, \cdots \mathbf{r}_{N}\right)\right|^{2} \underbrace{d \mathbf{r}_{2} d \mathbf{r}_{3} \cdots d \mathbf{r}_{N}}_{N-1}
$$

que representa a probabilidade de encontrar qualquer um dos $\mathrm{N}$ elétrons na vizinhança de $\mathbf{r}$. Dessa forma a energia eletrônica do estado fundamental pode ser escrita na forma 


$$
E_{0}=\left\langle\Psi_{0}(\mathbf{r})\left|\hat{T}_{N}+\hat{V}_{e}+\hat{V}_{e x t}\right| \Psi_{0}(\mathbf{r})\right\rangle=\left\langle\Psi_{0}(\mathbf{r})\left|\hat{T}_{N}+\hat{V}_{e}\right| \Psi_{0}(\mathbf{r})\right\rangle+\int \rho(\mathbf{r}) v(\mathbf{r}) d \mathbf{r}
$$

onde se observa uma relação entre o potencial externo e a densidade eletrônica do sistema.

A DFT mostra que a relação (2.8) é inversível, o que implica que densidade eletrônica pode ser usada, em princípio, para obter a função de onda e consequentemente, toda a informação quântica do sistema. Portanto, ao invés de buscar um objeto complexo como a função de onda do sistema (função de 3N coordenadas), é possível descrever o estado quântico de um sistema usando apenas a densidade eletrônica (uma função bem mais simples de apenas 3 coordenadas espaciais).

A DFT surgiu no ano de 1964, com a publicação de dois teoremas por Hohenberg e Khon (HK) [154]. Podemos resumir o conteúdo desses teoremas em três pontos:

(1) O potencial externo é um funcional único da densidade eletrônica do estado fundamental, $v(\mathbf{r})=v\left[\rho_{0}\right](\mathbf{r})$, a menos de uma constante aditiva. Assim, existe uma relação de unicidade entre a densidade eletrônica do estado fundamental $\rho_{0}(\mathbf{r})$ e o potencial externo $v(\mathbf{r})$.

A consequência imediata dessa afirmação é que a relação (2.8) pode ser invertida: é possível, em princípio, obter a função de onda $\Psi_{0}(\mathbf{r})$ a partir da densidade eletrônica do estado fundamental $\rho_{0}(\mathbf{r})$. Em outras palavras, a função de onda eletrônica do estado fundamental é um funcional único da densidade:

$$
\Psi_{0}(\mathbf{r})=\Psi_{0}\left[\rho_{0}\right](\mathbf{r})
$$

Essa é a essência do teorema de HK.

Como conseqüência, qualquer observável $O$ é completamente determinado pela densidade do estado fundamental,

$$
O_{0}=O\left[\rho_{0}\right]=\left\langle\Psi\left[\rho_{0}\right]|\hat{O}| \Psi\left[\rho_{0}\right]\right\rangle
$$

(2) Em particular, a energia eletrônica do estado fundamental é um funcional da 
densidade

$$
E_{0}=E\left[\rho_{0}\right]=\left\langle\Psi\left[\rho_{0}\right]|\hat{H}| \Psi\left[\rho_{0}\right]\right\rangle .
$$

O segundo teorema de HK assegura que, para o estado fundamental, a energia $E_{0}$ e a densidade $\rho_{0}$ de um sistema caracterizado pelo potencial $v_{0}(\mathbf{r})$ podem ser obtidos de um princípio variacional envolvendo a densidade:

$$
E\left[\rho_{0}\right] \leq E\left[\rho^{\prime}\right]
$$

onde $\rho_{0}$ é a densidade do estado fundamental e $\rho^{\prime}$ é uma densidade qualquer. É análogo a um princípio variacional para funções de onda.

(3) É possível então escrever a energia do sistema como sendo igual a

$$
E_{0}[\rho]=\left\langle\Psi[\rho]\left|\hat{T}+\hat{V}_{e}+\hat{V}_{e x t}\right| \Psi[\rho]\right\rangle=F[\rho]+\int d^{3} \mathbf{r} \rho(\mathbf{r}) v(\mathbf{r})
$$

onde $F[\rho]$ é um funcional universal, no sentido que não depende do potencial externo $v(\mathbf{r})$. Seja para átomos, sólidos ou moléculas, o potencial de repulsão eletrônica $V_{e}$ é o mesmo. Já o funcional $V_{\text {ext }}[\rho]$ depende da forma funcional do potencial externo $v(\mathbf{r})$, que é específico para cada sistema.

Uma vez especificado o potencial externo $v(\mathbf{r})$, a energia do sistema pode ser obtida a partir de uma equação do tipo Euler-Lagrange,

$$
\frac{\delta}{\delta \rho(\mathbf{r})} E[\rho(\mathbf{r})]=0
$$

A prova dos teoremas de HK podem ser encontradas nos livros texto sobre DFT (ver, por exemplo [145]) e não será reprozida aqui. Embora os teoremas de HK provem a existência de $F[\rho]$, não há um guia explícito de como sua forma funcional pode ser obtida.

\subsubsection{Equações de Khon-Sham}

A abordagem de Kohn-Sham (KS) [155] para a obtenção do funcional universal, foi proposta pouco tempo depois da publicação dos teoremas de HK. Ainda hoje é a 
formulação mais usada na aplicação da DFT em sistemas de muitos elétrons e responsável direta pelo imenso sucesso alcançado pela teoria. O método de KS consiste na introdução de um sistema fictício de elétrons não interagentes construído de forma a possuir a mesma densidade do sistema real de interesse.

No caso em que não se considera a interação entre os elétrons $\left(V_{e}=0\right)$ o sistema é caracterizado pelo Hamiltoniano $\hat{H}_{s}$ (o índice "s" subscrito vem do inglês singleparticle),

$$
\hat{H}_{s}=\hat{T}+\hat{V}_{s}=\sum_{j=1}^{N}\left(-\frac{1}{2} \nabla_{j}^{2}+v_{s}\left(\mathbf{r}_{j}\right)\right) .
$$

onde $v_{s}\left(\mathbf{r}_{j}\right)$ é um potencial externo de uma partícula.

Para esse sistema, os elétrons são independentes, e a função de onda do estado fundamental pode ser representada por um determinante de Slater,

$$
\Psi_{S}\left(\mathbf{r}_{1}, \mathbf{r}_{2}, \ldots, \mathbf{r}_{N}\right)=\frac{1}{\sqrt{N !}}\left|\begin{array}{cccc}
\varphi_{i}\left(\mathbf{r}_{1}\right) & \varphi_{j}\left(\mathbf{r}_{1}\right) & \cdots & \varphi_{k}\left(\mathbf{r}_{1}\right) \\
\varphi_{i}\left(\mathbf{r}_{2}\right) & \varphi_{j}\left(\mathbf{r}_{2}\right) & \cdots & \varphi_{k}\left(\mathbf{r}_{2}\right) \\
\vdots & \vdots & \vdots & \vdots \\
\varphi_{i}\left(\mathbf{r}_{N}\right) & \varphi_{j}\left(\mathbf{r}_{N}\right) & \cdots & \varphi_{k}\left(\mathbf{r}_{N}\right)
\end{array}\right|
$$

onde os orbitais de uma partícula $\varphi_{j}(\mathbf{r})$ satisfazem a equação de Schrödinger

$$
\left(-\frac{1}{2} \nabla_{j}^{2}+v_{s}\left(\mathbf{r}_{j}\right)\right) \varphi_{j}(\mathbf{r})=\varepsilon_{j} \varphi_{j}(\mathbf{r}) .
$$

Assim, a densidade do estado fundamental pode ser obtida pela expressão

$$
\rho_{s}=\sum_{j=1}^{N}\left|\varphi_{j}(\mathbf{r}, \sigma)\right|^{2}
$$


e a energia cinética como sendo igual a

$$
\hat{T}=\hat{T}_{s}\left[\rho_{s}\right]=-\frac{1}{2} \sum_{j=1}^{N}\left\langle\varphi_{j}\left|\nabla_{j}^{2}\right| \varphi_{j}\right\rangle .
$$

Soluções para o problema (2.18) podem ser encontradas por meio de um procedimento autoconsistente semelhante ao usado no método de HF (maiores detalhes na seção 2.4). Khon e Sham perceberam que poderiam usar esse sistema não interagente para mapear o problema original. Para isso basta reescrever o funcional energia como sendo igual a

$$
E[\rho]=T[\rho]+V[\rho]+\int d^{3} \mathbf{r} \rho(\mathbf{r}) v(\mathbf{r})=T_{s}[\rho]+\int d^{3} \mathbf{r} \rho(\mathbf{r}) v_{s}(\mathbf{r})+E_{H}+E_{x c}
$$

onde $E_{H}$ é o termo de energia de Hartree (que representa a interação Coulombiana clássica entre os elétrons),

$$
E_{H}=\frac{1}{2} \int d^{3} \mathbf{r} \int d^{3} \mathbf{r}^{\prime} \frac{\rho(\mathbf{r}) \rho\left(\mathbf{r}^{\prime}\right)}{\left|\mathbf{r}-\mathbf{r}^{\prime}\right|}
$$

e todos os outros termos são agrupados em um termo denominado a energia de troca e correlação

$$
E_{x c}[\rho]=T[\rho]-T_{s}[\rho]+V_{e}[\rho]-\int d^{3} \mathbf{r} \int d^{3} \mathbf{r}^{\prime} \frac{\rho(\mathbf{r}) \rho\left(\mathbf{r}^{\prime}\right)}{\left|\mathbf{r}-\mathbf{r}^{\prime}\right|}
$$

O princípio variacional (Equação 2.15) aplicado a expressão para a energia de KS permite chegar a expressão para o potencial externo

$$
v_{s}[\rho](\mathbf{r})=v[\rho](\mathbf{r})+\int d^{3} \mathbf{r}^{\prime} \frac{\rho\left(\mathbf{r}^{\prime}\right)}{\left|\mathbf{r}-\mathbf{r}^{\prime}\right|}+v_{x c}[\rho](\mathbf{r}),
$$

onde o potencial de troca e correlação é definido por

$$
v_{x c}[\rho](\mathbf{r})=\frac{\delta E_{x c}}{\delta[\rho](\mathbf{r})} .
$$

Infelizmente o termo de troca e correlação não é conhecido e apenas formas 
funcionais aproximadas são usadas na prática. Caso esse termo fosse conhecido, e energia do sistema poderia ser calculada exatamente! A grande vantagem do método de Khon-Sham é que a forma dos termos que mais contribuem para a energia (os termos de Hartree, energia cinética) são conhecidos. Os termos agrupados no termo de troca e correlação, embora desconhecidos, contribuem menos para a energia do sistema. Ainda sim, esse termo não pode ser desprezado pois ali reside toda a contribuição para a energia de correlação do sistema.

\subsubsection{Aproximações para o potencial de troca-correlação}

Em princípio, não existe uma forma sistemática de desenvolver uma aproximação para os funcionais de troca e correlação. Uma sistematização no desenvolvimento de novos funcionais foi realizada por John Perdew $[159,160]$, em um esquema chamado por ele de a escada de Jacó para os funcionais (ver Tabela 2.1).

Tabela 2.1: Escada de Jacó dos funcionais

O Paraíso da Química Quântica

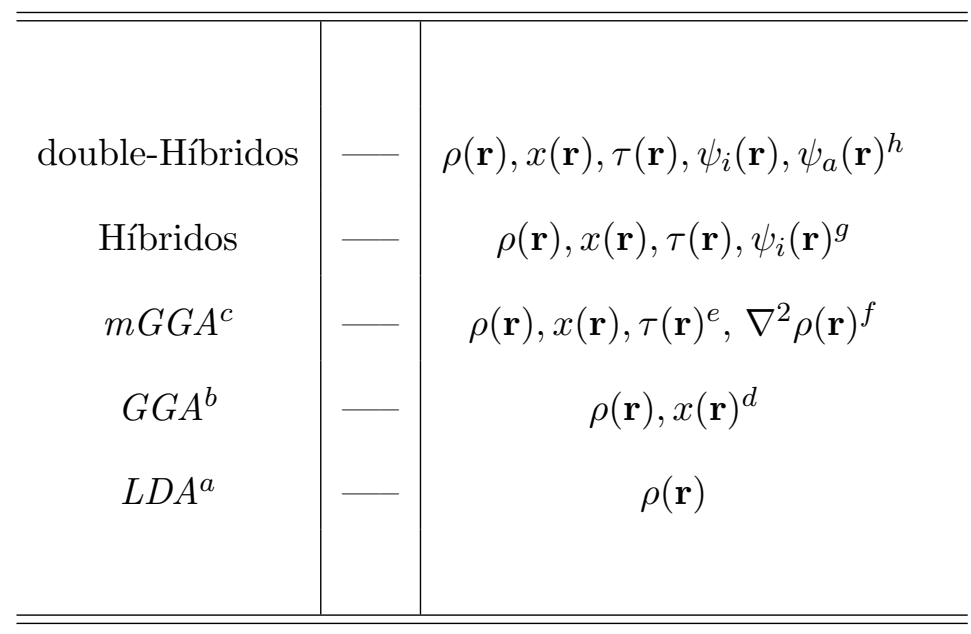

O mundo terrestre: modelo de Hartree

${ }^{a} L D A$, ver texto. ${ }^{b} G G A$, ver texto. ${ }^{c}$ Meta $G G A .{ }^{d}$ Gradiente reduzido $x(\mathbf{r})=|\vec{\nabla} \rho(\mathbf{r})| / \rho^{4 / 3}(\mathbf{r})$.

${ }^{e}$ Energia cinética local $\tau(\mathbf{r})=\sum_{p} n_{p} \psi_{p}(\mathbf{r}) \nabla^{2} \psi_{p}(\mathbf{r}) .{ }^{f}$ Densidade local da energia cinética, $\tau(\mathbf{r})$, laplaciano da densidade de carga, $\nabla^{2} \rho(\mathbf{r}),{ }^{g}$ Orbitais ocupados. ${ }^{h}$ Orbitais virtuais. 
Nessa classificação, cada nível de aproximação corresponde a um degrau da escada que, sistematicamente, vai aumentando em precisão, rumo ao "paraíso" da acurácia química. A aproximação mais simples (primeiro degrau) consiste em usar a densidade de um gás homogêneo de elétrons, conhecida como aproximação de densidade local (LDA, do inglês Local Density Approximation):

$$
E_{x c}^{L D A}=\int d^{3} \mathbf{r} \rho(\mathbf{r}) \varepsilon_{x c}[\rho(\mathbf{r})], \varepsilon_{x c}[\rho(\mathbf{r})]=\varepsilon_{x}[\rho(\mathbf{r})]+\varepsilon_{C}[\rho(\mathbf{r})]
$$

onde

$$
\varepsilon_{x}[\rho(\mathbf{r})]=-\frac{3}{4}\left(\frac{3 \rho(\mathbf{r})}{\pi}\right)^{\frac{1}{3}}
$$

é a energia de troca de Dirac/Slater para um gás de elétrons.

Já o termo de correlação, $\varepsilon_{c}[\rho(\mathbf{r})]$, possui uma forma mais complexa e em geral é obtido por interpolação a dados obtidos com Monte Carlo Quântico. Em geral, a aproximação LDA não é capaz de produzir resultados satisfatórios em sistemas moleculares.

Os funcionais do degrau seguinte levam em consideração a variação espacial da densidade $(g=|\nabla \rho|)$ e são conhecidos como funcionais com a Aproximação de Gradiente Generalizado (GGA, do inglês Generalized Gradient Approximation), que melhoraram consideravelmente a acurácia da DFT em moléculas. Novamente, o termo xc possui uma expressão para o termo de troca e outro para o termo de correlação. As formas mais conhecidas para o termo de correlação são: LYP (Lee, Yang e Parr, 1988) [161]; PW91 (Perdew e Wang, 1991) [162]; P86 (Perdew, 1986) [163].

O termo de troca na aproximação GGA tem a forma,

$$
E_{x}^{G G A}=E_{x}^{L D A}-\sum_{\sigma} \int F\left(s_{\sigma}\right) \rho_{\sigma}^{4 / 3}(\mathbf{r}) d \mathbf{r},
$$

onde

$$
s_{\sigma}=\frac{|\nabla \rho|}{\rho^{4 / 3}} .
$$

As duas escolhas mais comuns para o termo $F(s)$ são a de Becke 1988 (B88) [164] e a de Perdew (1986) [165]. Uma diferença importante entre elas é que Becke introduz um parâmetro obtido a partir de ajustes à densidade de um grande número de átomos, 
enquanto Perdew não usa nenhum parâmetro empírico.

Posteriormente Perdew, Burke e Ernzerhof desenvolvem o funcional PBE [166]. Este funcional, que também não usa parâmetros empíricos, tornou-se rapidamente uma escolha padrão nos cálculos de estrutura eletrônica, sobretudo de sólidos, capaz de descrever de forma adequada uma grande quantidade propriedades e sistemas.

Os funcionais do próximo degrau da escada são conhecidos como meta-GGA e têm forma

$$
E_{x c}^{m G G A}=\int d^{3} \mathbf{r} e_{x c}[\rho(\mathbf{r}), g, l, \tau]
$$

com dependência da densidade, do gradiente da densidade e do laplaciano da densidade,

$$
l=\nabla^{2} \rho
$$

além da energia cinética,

$$
\tau=\frac{1}{2} \sum_{k}^{o c c}\left|\nabla \phi_{k}(\mathbf{r})\right|^{2}
$$

\subsubsection{Funcionais híbridos}

Os funcionais híbridos surgiram como uma tentativa de melhorar os resultados de DFT com a combinação de funcionais puros com uma taxa do termo de troca de HF. Em 1993, Becke sugeriu o funcional B3LYP [167], combinando a aproximação GGA (troca B88 e correlação LYP), aproximação LDA (SVWN) e HF por meio de três parâmetros,

$$
E_{x c}^{B 3 L Y P}=a_{0} E_{x}^{H F}+\left(1-a_{0}\right) E_{x}^{\text {Slater }}+a_{x} E_{x}^{B 88}+a_{C} E_{x}^{L Y P}+\left(1-a_{C}\right) E_{C}^{V W N}
$$

onde os parâmetros $a_{0}=0.2, a_{x}=0.72$ e $a_{c}=0.81$ foram obtidos a partir ajustes a resultados experimentais. Esse funcional se tornou rapidamente o mais usado na área de química quântica, já que é capaz de produzir resultados razoáveis para um grande número de propriedades, desde geometrias de equilíbrio a propriedades termoquímicas. Por essa razão, os funcionais híbridos foram os grandes responsáveis pela popularização da DFT na área de química quântica. Neste trabalho, usamos o funcional B3LYP para o cálculo das propriedades do estado fundamental, geometrias de equilíbrio, espectro 
vibracional, propriedades magnéticas, etc.

\subsubsection{Correções de longo alcance}

Apesar do sucesso dos funcionais híbridos, eles falham em descrever uma série de sistemas importantes, como estados de Rydberg e, principalmente, as excitações de transferência de carga, que são um ponto central neste trabalho. Para lidar com esse problema, os cálculos de energia de excitação precisam ser realizados usando funcionais com correção de longo alcance (LC) [75, 168]. Nesses funcionais, o termo $1 / r_{12}$ é separado em uma parte de curto alcance (usando $E_{x}$ de DFT) e outra de longo alcance (que usa $E_{x}$ de HF). As contribuição de cada parte é modulada por uma função erro do tipo gaussiana $\operatorname{erf}\left(\mu r_{12}\right)$.

$$
\frac{1}{r_{12}}=\frac{1-\operatorname{erf}\left(\mu r_{12}\right)}{r_{12}}+\frac{\operatorname{erf}\left(\mu r_{12}\right)}{r_{12}}
$$

O parâmetro $\mu$ permite controlar a contribuição de HF, que aumenta gradativamente com a distância. As correções foram propostas inicialmente para funcionais puros (LC-BLYP, LC-PBE, etc) mas uma generalização foi realizada para o funcional híbrido B3LYP, chamada de CAM-B3LYP [76]. (o termo CAM vem do inglês CoulombAttenuating Method), com a adição de dois parâmetros extras $\alpha$ e $\beta$.

$$
\frac{1}{r_{12}}=\frac{1-\left[\alpha+\beta \cdot \operatorname{erf}\left(\mu r_{12}\right)\right]}{r_{12}}+\frac{\alpha+\beta \cdot \operatorname{erf}\left(\mu r_{12}\right)}{r_{12}}
$$

onde $0 \leq \alpha+\beta \leq 1,0 \leq \alpha \leq 1$ e $0 \leq \beta \leq 1$. A expressão usual pode ser obtida colocando $\alpha=0.2$ e $\beta=0.0$ na mistura entre $E_{H F}^{x}$ e $E_{\text {Slater }}^{x}$. O esquema de correção original (LC) também é obtido com $\alpha=0.0$ e $\beta=1.0$. No método CAM-B3LYP, o valor dos parâmetros são $\alpha=0.19, \alpha+\beta=0.65$ e $\mu=0.33$.

Apesar do sucesso da DFT, ela só é capaz de descrever corretamente propriedades obtidas a partir da energia do estado fundamental do sistema. Infelizmente, ainda não foi possível criar uma formulação variacional para se obter a energia dos estados excitados do sistema a exemplo do que propõe o segundo teorema de KS. Mas é possível obter algumas propriedades dependentes de estados excitados, sobretudo aquelas que dependem da resposta do sistema a campos externos, usando a TDDFT. 


\subsection{Solução das equações de Kohn-Sham}

Vimos (ver seção 2.3.1) que as soluções do problema não interagente,

$$
\hat{H}_{s}=\hat{T}+\hat{V}_{s}=\sum_{j=1}^{N}\left(-\frac{1}{2} \nabla_{j}^{2}+v_{s}\left(\mathbf{r}_{j}\right)\right),
$$

podem ser representada por um determinante de Slater,

$$
\Psi_{S}\left(\mathbf{r}_{1}, \mathbf{r}_{2}, \ldots, \mathbf{r}_{N}\right)=\frac{1}{\sqrt{N !}}\left|\begin{array}{cccc}
\varphi_{i}\left(\mathbf{r}_{1}\right) & \varphi_{j}\left(\mathbf{r}_{1}\right) & \cdots & \varphi_{k}\left(\mathbf{r}_{1}\right) \\
\varphi_{i}\left(\mathbf{r}_{2}\right) & \varphi_{j}\left(\mathbf{r}_{2}\right) & \cdots & \varphi_{k}\left(\mathbf{r}_{2}\right) \\
\vdots & \vdots & \vdots & \vdots \\
\varphi_{i}\left(\mathbf{r}_{N}\right) & \varphi_{j}\left(\mathbf{r}_{N}\right) & \cdots & \varphi_{k}\left(\mathbf{r}_{N}\right)
\end{array}\right|
$$

onde os orbitais de uma partícula $\varphi_{j}(\mathbf{r})$, satisfazem a equações de Khon-Sham

$$
\left(-\frac{1}{2} \nabla_{j}^{2}+v_{s}\left(\mathbf{r}_{j}\right)\right) \varphi_{j}(\mathbf{r})=\varepsilon_{j} \varphi_{j}(\mathbf{r})
$$

O método usado na solução das equações de Kohn-Sham consiste em utilizar uma expansão dos orbitais moleculares em uma base finita conhecida, transformando o problema em uma equação algébrica que pode ser resolvida por procedimentos usuais de diagonalização matricial. Introduzindo um conjunto $\left\{\phi_{\nu}(\mathbf{r})\right\}$ de $\mathrm{K}$ funções base, então

$$
\varphi_{\nu}(\mathbf{r})=\sum_{\nu=1}^{K} C_{\nu i} \phi_{\nu}(\mathbf{r}) . \quad i=1,2, \ldots, K .
$$

As funções de bases mais usadas são compostas por orbitais de Slater, funções gaussianas e ondas planas. 


\subsection{Funções Base}

\subsubsection{Bases gaussianas e funções de Slater}

Dois tipos básicos de conjunto de funções base [169] (também chamados de orbitais atômicos) são amplamente utilizadas nos cálculos da estrutura eletrônica molecular: funções do tipo Slater STO (Slater-type Orbital) [170] e funções do tipo gaussianas GTO (Gaussian-type Orbital) [171] .

As STOs têm a forma

$$
\phi_{\zeta, n, l, m}(r, \theta, \varphi)=N Y_{l, m}(\theta, \varphi) r^{n-1} e^{-\zeta r}
$$

onde $N$ é uma constante de normalização e os $Y_{l m}$ são harmônicos esféricos. A principal característica destes orbitais é o decaimento exponencial, espelhado no comportamento da solução exata da equação de Schrödinger para um átomo hidrogenóide, que favorece a rápida convergência dos resultados usando um número reduzido de funções base. Entretanto, a desvantagem das STOs é que integrais de 1 e 2 elétrons na maior parte dos casos não possuem solução analítica e seus cálculos numéricos demandam grande esforço computacional.

A alternativa às STOs são as GTOs, que possuem a seguinte forma funcional

$$
\phi_{\zeta, n, l, m}(r, \theta, \varphi)=N Y_{l, m}(\theta, \varphi) r^{2 n-2-l} e^{-\zeta r^{2}},
$$

em coordenadas esféricas. Elas também podem ser escritas em coordenadas cartesianas,

$$
\phi_{\zeta, l_{x}, l_{y}, l_{z}}(x, y, z)=N x^{l_{x}} y^{l_{y}} z^{l_{z}} e^{-\zeta^{2}}
$$

de forma que a soma de $l_{x}, l_{y}$ e $l_{z}$ permite classificar o tipo de orbital. Quando $l_{x}=l_{y}=l_{z}=0$ o orbital é uma gaussiana tipo s; quando $l_{x}+l_{y}+l_{z}=1$, gaussiana tipo p; quando $l_{x}+l_{y}+l_{z}=2$, gaussiana tipo d; e assim por diante.

A facilidade no cálculo das integrais envolvendo produtos de GTOs é a principal vantagem deste tipo de base. A desvantagem é que o decaimento gaussiano não reproduz adequadamente as características dos orbitais atômicos à curtas e nem à longas distâncias, o que torna neste aspecto este tipo de função base inferior às STOs. 
Para contornar este problema, em geral um pacote de funções gaussianas é usado para representar um orbital molecular, conhecido como função gaussiana contraída. Cada função base contraída $\varphi_{i}$, é construída como uma combinação linear

$$
\phi_{i}=\sum_{i=1}^{L} d_{j i} g_{j}
$$

de outras funções, chamadas de gaussianas primitivas. Elas são representadas por $g_{j}$, têm a forma gaussiana dada pela equação (2.42) e são centradas no mesmo núcleo atômico. As GTOs são usadas na maioria dos cálculos de estrutura eletrônica, já que a eficiência computacional no cálculos das integrais é bem maior que no caso das STOs. Existe na literatura, uma quantidade muito grande deste tipo de funções base, desenvolvidas especialmente para o cálculo das mais variadas propriedades [172].

Uma vez decidida a forma das funções base (GTO ou STO), resta ainda a escolha prática do número de funções base a ser para representar cada orbital atômico. A escolha mais simples é conhecida como a base mínima, onde apenas uma função contraída é usada para representar cada orbital. Por exemplo, se usa uma única função base no caso de um átomo de $\mathrm{H}$ ou He (orbitais 1s); 5 funções base no caso de átomos de Li a Ne (1 orbital 1s, 1 orbital 2s, e 3 orbitais 2p); e assim por diante. Esta simplificação em geral é insatisfatória para a obtenção de resultados acurados e uma melhora significativa nos resultados é conseguida aumentando-se o número de funções base para cada orbital. Bases DZ (double-zeta) usam duas, TZ (triple-zeta) usam três, e assim por diante.

As funções base do tipo SV(split-valence) buscam um compromisso intermediário entre a inadequação das base mínima e o alto custo computacional das funções $D Z$ ou $T Z$, atribuindo a base mínima para os orbitais de caroço e bases duplas para os orbitais de valência. Jonh Pople criou série de funções deste tipo, designadas genericamente por $X$-YZG (3-21G, 6-31G, 6-311Getc), onde $X$ designa o número de funções primitivas usadas para ajustar os orbitais de caroço e os números restantes $Y Z$ significam que a região de valência é dividida em duas partes com Y primitivas na primeira Z na segunda.

Nesta categoria melhorias ainda podem ser realizadas com a inclusão de funções adicionais de polarização e funções difusas. Funções de polarização melhoram a 
descrição da deformação dos orbitais que participam da ligação. A existência de um asterisco $(*)$ — ou "(d)" — logo após a letra G, como em 6-311G* — ou 6-31G(d) - indica que funções $d$ foram adicionadas aos átomos pesados (não hidrogênio). Dois asteriscos $\left({ }^{* *}\right)$ indicam que funções $p$ também foram adicionadas aos átomos de hidrogênio. Funções difusas, que neste esquema são representadas por sinal de adição $(+)$, melhoram a descrição da densidade eletrônica em regiões relativamente afastadas do núcleo. A função base $6-311+G(d, p)$ consiste, portanto, de uma função com qualidade TZ na valência, com função difusa e polarização nos átomos pesados e no hidrogênio.

As STO e as GTO são em geral centradas nas posições dos núcleos atômicos ("átomos"), i.e $r \rightarrow r-R_{I}$ nas equações (2.41) e (2.42), o que leva a aproximação conhecida como LCAO (do inglês "Linear Combination of Atomic Orbitals")) que permite resolver as equações diferenciais Kohn-Sham algebricamente.

Dunnning e Huzinaga desenvolveram o conjunto conhecido como cc-PVXZ (correlation-consistent $X$ Zeta, onde $X=D$ (double), $T$ (triple), $Q$ (quadruple), 5, 6, .. etc, e os coeficientes são otimizados para cálculos que exijam correlação eletrônica, incluindo naturalmente funções de polarização. $V X Z$ indica que $X$ funções são usadas, mas apenas para os orbitais de valência. Funções difusas a todos os átomos em geral também são adicionadas, indicadas pelo prefixo "aug", como em aug-cc-PVDZ.

\subsubsection{Bases de ondas planas}

Um abordagem diferente e que tem suas raízes na física do estado sólido, onde a periodicidade das redes cristalinas sugere o uso de bases de ondas planas. Ondas planas são definidas como

$$
\phi_{\mathbf{G}}^{P W}(\mathbf{r})=N \exp [i \mathbf{G} \cdot \mathbf{r}]
$$

onde a normalização é dada por $N=1 / \Omega ; \Omega$ é o volume da célula unitária e $\mathbf{G}$ um vetor da rede recíproca. Como ondas planas formam um conjunto completo, elas podem ser usadas para representar os orbitais conforme a Equação (2.39),

$$
\varphi(\mathbf{r})=\frac{1}{\Omega} \sum_{\mathbf{G}<\mathbf{G}_{\max }} C_{i}(\mathbf{G}) \exp [i \mathbf{G} \cdot \mathbf{r}]
$$


onde $\mathbf{G}_{\max }$ é definido a partir de uma energia cinética de corte $E_{c u t}$

$$
\frac{1}{2}\left|\mathbf{G}_{\max }\right|^{2} \leq E_{\text {cut }} .
$$

O uso de ondas planas tem vantagens como ortogonalidade e independência das posições atômicas. Além disso, melhorias na descrição da função podem ser alcançadas bastando aumentar o raio de corte. Entretanto, em geral um número bem maior de funções ondas é necessário para representar uma molécula se comparado ao uso das funções gaussianas. Isso é particularmente dramático para o caso dos elétrons de caroço, cuja rápida oscilação da função de onda exige um número muito grande de ondas planas. Por isso é muito comum introduzir um pseudopotencial para descrever os elétrons de caroço e tratando explicitamente apenas os elétrons de valência. Neste trabalho, usamos esse tipo de base apenas na etapa de dinâmica por primeiro princípios, que será tratada no próximo capítulo. 


\subsection{Teoria do funcional da densidade dependente do tempo}

Durante muito tempo após a publicação dos teoremas de HK buscou-se uma forma de se calcular propriedades dos estados excitados usando a DFT . Mas nenhuma abordagem baseada na DFT foi capaz de produzir aproximações razoáveis para propriedades como energias de excitação, por exemplo (maiores detalhes podem ser encontrados no capítulo 2 da referência [148]). Os cálculos das propriedades dos estados excitados se tornou possível com o surgimento da teoria do funcional da densidade dependente do tempo (TDDFT) [173].

A TDDFT permite tratar sistemas sujeitos à potenciais com dependência temporal e, a exemplo da DFT para o estado fundamental, tornou-se uma das teorias de maior sucesso no cálculo de propriedades dos estados excitados de sistemas moleculares, como por exemplo, energias de excitação, força de oscilador, geometrias do estado excitado, etc.

A extensão do teorema de KS para sistemas dependentes do tempo é conhecida como teorema de Runge-Gross (RG) [156]. Na TDDFT, não é possível estabelecer um princípio variacional para a energia. Existe no entanto, uma grandeza análoga, que é a ação

$$
\mathcal{A}=\int_{t_{0}}^{t_{1}}\left\langle\Phi(t)\left|i \frac{\partial}{\partial t}-\hat{H}(t)\right| \Phi(t)\right\rangle
$$

onde $\Phi(t)$ é uma função de N-corpos. Essa grandeza permite definir um princípio de ação estacionária, de forma que as soluções $\Phi(\mathbf{r}, t)$ que tornam a ação estacionária são soluções da equação de Schrödinger dependente do tempo. RG usam a ação como funcional da densidade dependente do tempo $\rho(\mathbf{r}, t)$ para estabelecer a relação de unicidade com potencial externo $V(\mathbf{r}, t)$ e, consequentemente, estender os teoremas de KS para versões dependentes do tempo. 


\subsection{O problema dependente do tempo}

A equação de Schrödinger dependente do tempo é dada por

$$
i \frac{\partial}{\partial t} \Psi_{j}\left(\mathbf{r}_{1}, \ldots, \mathbf{r}_{N}, t\right)=\hat{H}(t) \Psi_{j}\left(\mathbf{r}_{1}, \ldots, \mathbf{r}_{N}, t\right)
$$

onde o Hamiltoniano dependente do tempo é definido por

$$
\hat{H}(t)=\hat{T}+\hat{V}(t)+\hat{V}_{e}
$$

O hamiltoniano dependente do tempo se assemelha ao do caso estático (2.6) nos termos $\hat{T}$ and $\hat{V}_{e}$. mas o potencial externo possui dependência temporal explícita:

$$
\hat{V}(t)=\sum_{j=1}^{N} v\left(\mathbf{r}_{j}, t\right) .
$$

A equação de Schrödinger dependente do tempo (2.48) é formalmente um problema de valor inicial. Sejam $t_{0}$ o tempo inicial (em geral, $t_{0}=0$ ), e $\Psi\left(t_{0}\right) \equiv \Psi_{0}$ a função de onda inicial (note que não precisa ser a função de onda do estado fundamental). Esse estado é então propagado no tempo, de forma a descrever a evolução temporal do sistema sujeito à influência do potencial dependente do tempo $v(\mathbf{r}, t)$. Em muitas situações de interesse prático, o potencial de uma partícula dependente do tempo poder ser escrito como

$$
v(\mathbf{r}, t)=v_{0}(\mathbf{r})+\theta\left(t-t_{0}\right) v_{1}(\mathbf{r}, t)
$$

onde $\theta\left(t-t_{0}\right)$ é a função degrau de Heavside,

$$
\theta\left(t-t_{0}\right)= \begin{cases}0, & t<t_{0} \\ 1, & t>t_{0}\end{cases}
$$

Assim, o potencial estático é igual a $v_{0}$ até o tempo $t_{0}$ quanto um potencial adicional $v_{1}(t)$ com dependência temporal explícita é ligado.

A função de onda dependente do tempo permite calcular qualquer observável de 
interesse,

$$
O(t)=\langle\Psi(t)|\hat{O}| \Psi(t)\rangle
$$

onde $O(t)$ é o valor esperado do operador Hermiteano $\hat{O}$ que corresponde a um observável.

\subsection{O formalismo da TDDFT}

\subsubsection{O teorema de Runge-Gross}

O fundamento da DFT para o estado fundamental é o teorema de HohenbergKohn, discutido na Seção 2.3. A correspondência 1:1 entre a densidade do estado fundamental e o potencial externo torna possível construir funcionais da densidade e determinar as propriedades do estado fundamental exatamente (em princípio) por meio de soluções autoconsistentes das equações de Kohn-Sham.

O caso dependente do tempo difere do estado fundamental por duas razões importantes. Primeiro, no caso dependente do tempo não há um princípio variacional para a energia. Em segundo lugar, a equação de Schrödinger (2.48) é agora um problema de valor inicial, então é preciso sempre ter em mente um estado inicial de referência.

A prova da existência do mapeamento 1:1 no caso dependente do tempo foi inicialmente dada por Runge e Gross em 1984 [156]. O teorema de Runge-Gross assume potenciais que podem ser escritos na forma de uma série de potencias ao redor de $t_{0}$ :

$$
v(\mathbf{r}, t)=\sum_{k=0}^{\infty} \frac{v_{k}(\mathbf{r})}{k !}\left(t-t_{0}\right)^{k} .
$$

Para esse tipo de potencial, é possível provar uma correspondência 1:1 da forma:

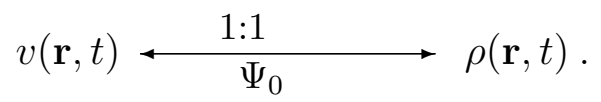

Da mesma forma que na DFT para o estado fundamental, a correspondência 1:1 (2.55) permite escrever o potencial externo como um funcional da densidade:

$$
v(\mathbf{r}, t)=v\left[\rho, \Psi_{0}\right](\mathbf{r}, t)
$$


Embora formalmente exista a dependência do estado inicial, ela pode ser contornada tomando o estado fundamental como referência, i.e., $\Psi_{0}=\Psi_{\mathrm{gs}}$.

\subsubsection{O formalismo de Kohn-Sham dependente do tempo}

A densidade dependente do tempo, $\rho(\mathbf{r}, t)$, para um sistema não interagente descrito por $N$ orbitais de uma partícula, é dada por:

$$
\rho(\mathbf{r}, t)=\sum_{j=1}^{N}\left|\varphi_{j}(\mathbf{r}, t)\right|^{2} .
$$

Os orbitais $\varphi_{j}(\mathbf{r}, t)$ satisfazem a equação de Kohn-Sham dependente do tempo:

$$
i \frac{\partial}{\partial t} \varphi_{j}(\mathbf{r}, t)=\left[-\frac{\nabla^{2}}{2}+v_{s}(\mathbf{r}, t)\right] \varphi_{j}(\mathbf{r}, t)
$$

onde o potencial efetivo dependente do tempo é dado por

$$
v_{s}\left[\rho, \Psi_{0}, \Phi_{0}\right](\mathbf{r}, t)=v(\mathbf{r}, t)+v_{\mathrm{H}}(\mathbf{r}, t)+v_{\mathrm{xc}}\left[\rho, \Psi_{0}, \Phi_{0}\right](\mathbf{r}, t) .
$$

Aqui, $v(\mathbf{r}, t)$ é o potencial externo dependente do tempo, da forma (2.51). O potencial de Hartree dependente do tempo,

$$
v_{\mathrm{H}}(\mathbf{r}, t)=\int d^{3} r^{\prime} \frac{\rho\left(\mathbf{r}^{\prime}, t\right)}{\left|\mathbf{r}-\mathbf{r}^{\prime}\right|},
$$

depende instantaneamente apenas da densidade dependente do tempo. O potencial xc depende formalmente da densidade, do estado inicial $\Psi_{0}$ exato do sistema interagente, e da condição inicial do sistema de Kohn-Sham, $\Phi_{0}$.

\subsubsection{O potencial de troca-correlação dependente do tempo}

O potencial xc dependente do tempo é formalmente um funcional da densidade bem como dos estados usados como condição inicial, $v_{\mathrm{xc}}\left[\rho, \Psi_{0}, \Phi_{0}\right](\mathbf{r}, t)$. Na prática as situações de interesse residem em sistemas cuja condição inicial sejam o estado fundamental. Nesse caso as coisas simplificam consideravelmente: graças ao teorema 
de Hohenberg-Kohn para a DFT do estado fundamental o os estados iniciais tornam-se funcionais da densidade inicial, e o funcional xc pode ser escrito como funcional da densidade apenas, $v_{\mathrm{xc}}[\rho](\mathbf{r}, t)$.

Entretanto, a dependência do potencial xc com a densidade é não local: o potencial xc em um ponto do espaço tempo $(\mathbf{r}, t)$ depende de densidades em todos os pontos do espaço e em todos os tempos anteriores, $\rho\left(\mathbf{r}^{\prime}, t^{\prime}\right)$, onde $t^{\prime} \leq t$ (o potencial não pode depender de uma densidade futura - isso violaria o princípio de causalidade).

É necessário então simplificar a forma do potencial xc. A aproximação mais usada para o potencial xc é a aproximação adiabática:

$$
v_{\mathrm{xc}}^{\mathrm{A}}(\mathbf{r}, t)=\left.v_{\mathrm{xc}}^{\mathrm{gs}}\left[\rho_{0}\right](\mathbf{r})\right|_{\rho_{0}(\mathbf{r})=\rho(\mathbf{r}, t)},
$$

onde $v_{\mathrm{xc}}^{\mathrm{gs}}$, o potencial xc para o estado fundamental definido na Equação (2.18), é calculado usando a densidade dependente do tempo instantânea. A Equação (2.61) torna-se exata para um sistema infinitamente lento e que permanece no estado fundamental durante todo o tempo. Apesar disso, a aproximação adiabática funciona muito bem em várias situações práticas.

\subsubsection{TDDFT e resposta linear}

Encontrar uma solução para o problema dependente do tempo, mesmo para as equações de TD-KS é uma tarefa muito mais árdua comparado ao caso estacionário. Felizmente para muitas aplicações práticas, obter tal solução nem sempre é necessário ou desejável. Uma série de propriedades moleculares como polarizabilidade ou energias de excitação podem ser calculadas usando a teoria de resposta.

A teoria de resposta linear é um método amplamente usado, adequado para o tratamento de sistemas sujeitos á perturbações externas. Como veremos a seguir, a função resposta linear no caso molecular contém toda informação sobre as excitações eletrônicas. Vale ressaltar que também existe a possibilidade de buscar respostas de mais alta ordem, mas esse tipo de tratamento foge ao escopo deste trabalho. Aspectos formais da teoria de resposta pode ser encontrados nas referências [174,175]. Para uma dedução mais geral da teoria de resposta linear o leitor pode consultar o capítulo 7 da referência [148]. 


\subsubsection{Formalismo}

Em muitas situações de interesse prático, os sistemas são sujeitos a pequenas perturbações e, portanto, não ocorrem desvios acentuados em relação ao seu estado inicial. Isto acontece na maioria das aplicações em espectroscopia. Nesse caso, não é necessário a procurar a solução completa do problema dependente do tempo. Basta uma solução perturbativa.

Considere então um sistema inicialmente em seu estado fundamental e um potencial externo dependente do tempo que passa a agir no sistema a partir de um instante $t_{0}$ - veja a Equação (2.51). Entretanto, $v_{1}(\mathbf{r}, t)$ agora será tratado como uma pequena perturbação, que causa mudanças (pequenas) no sistema. Escrevendo em termos de série de potencias:

$$
\rho(\mathbf{r}, t)=\rho_{0}(\mathbf{r})+\rho_{1}(\mathbf{r}, t)+\rho_{2}(\mathbf{r}, t)+\ldots
$$

Aqui, $\rho_{0}$ é a densidade do estado fundamental, $\rho_{1}$ é a densidade resposta linear (a mudança em primeira ordem na densidade do sistema induzida pela perturbação $v_{1}$ ), $\rho_{2}$ é densidade resposta de segunda ordem (quadrática na perturbação $v_{1}$ ), e assim por diante. Se a perturbação é pequena o termo linear é dominante e os termos de ordem superior poder ser desprezados.

A função resposta linear do sistema pode ser formalmente escrita como sendo igual a

$$
\rho_{1}(\mathbf{r}, t)=\int_{-\infty}^{\infty} d t^{\prime} \int d^{3} r^{\prime} \chi\left(\mathbf{r}, t, \mathbf{r}^{\prime}, t^{\prime}\right) v_{1}\left(\mathbf{r}^{\prime}, t^{\prime}\right)
$$

Aqui, $\chi\left(\mathbf{r}, \mathbf{r}^{\prime}, t-t^{\prime}\right)$ é a função resposta densidade-densidade [148,175], definida como sendo igual a

$$
\chi\left(\mathbf{r}, t, \mathbf{r}^{\prime}, t^{\prime}\right)=-i \theta\left(t-t^{\prime}\right)\left\langle\Psi_{\mathrm{gs}}\left|\left[\hat{\rho}\left(\mathbf{r}, t-t^{\prime}\right), \hat{\rho}\left(\mathbf{r}^{\prime}\right)\right]\right| \Psi_{\mathrm{gs}}\right\rangle
$$

A função degrau $\theta\left(t-t^{\prime}\right)$ assegura que a resposta é causal, i.e., a resposta vem após a perturbação. A equação (2.64) mostra que função resposta é obtida a partir do estado fundamental $\Psi_{\mathrm{gs}}$, e envolve o comutador do operador densidade (na representação de interação). Daí, via teorema de Hohenberg-Kohn, ela é formalmente um funcional da densidade do estado fundamental, $\chi\left[\rho_{0}\right]$. Normalmente, é mais interessante obter a 
função resposta no domínio das frequências:

$$
\rho_{1}(\mathbf{r}, \omega)=\int d^{3} r^{\prime} \chi\left(\mathbf{r}, \mathbf{r}^{\prime}, \omega\right) v_{1}\left(\mathbf{r}^{\prime}, \omega\right)
$$

A transforma da de Fourier da função resposta (2.64) pode ser escrita na forma conhecida como representação de Lehmann $[148,175]$ :

$$
\begin{aligned}
\chi\left(\mathbf{r}, \mathbf{r}^{\prime}, \omega\right)=\sum_{n=1}^{\infty} & \left\{\frac{\left\langle\Psi_{\mathrm{gs}}|\hat{\rho}(\mathbf{r})| \Psi_{n}\right\rangle\left\langle\Psi_{n}\left|\hat{\rho}\left(\mathbf{r}^{\prime}\right)\right| \Psi_{\mathrm{gs}}\right\rangle}{\omega-\Omega_{n}+i \eta}\right. \\
& \left.-\frac{\left\langle\Psi_{\mathrm{gs}}\left|\hat{\rho}\left(\mathbf{r}^{\prime}\right)\right| \Psi_{n}\right\rangle\left\langle\Psi_{n}|\hat{\rho}(\mathbf{r})| \Psi_{\mathrm{gs}}\right\rangle}{\omega+\Omega_{n}+i \eta}\right\},
\end{aligned}
$$

onde fica subentendido o limite $\eta \rightarrow 0^{+}$.

$$
\Omega_{n}=E_{n}-E_{0}
$$

é a $n$-ésima energia de excitação do sistema. Essa representação é interessante pois mostra explicitamente que a função resposta tem pólos nas energias de excitação do sistema. A interpretação disso é que ao aplicar uma perturbação $v_{1}(\mathbf{r}, \omega)$ na frequência correspondente à energia de excitação uma resposta muito grande é induzida no sistema (e um pico aparece no espectro). Se a função resposta $\chi$ do sistema fosse conhecida, o cálculo da densidade resposta seria trivial: bastaria usar a expressão (2.65).

Na TDDFT, a densidade resposta linear pode ser calculada, em princípio exatamente, como a resposta de um sistema de Kohn-Sham não interagente sujeito à uma perturbação efetiva [176]:

$$
\rho_{1}(\mathbf{r}, t)=\int d t^{\prime} \int d^{3} r^{\prime} \chi_{s}\left(\mathbf{r}, t, \mathbf{r}^{\prime}, t^{\prime}\right) v_{1 s}\left(\mathbf{r}^{\prime}, t^{\prime}\right) .
$$

Aqui, $\chi_{s}\left(\mathbf{r}, \mathbf{r}^{\prime}, t-t^{\prime}\right)$ é a função densidade-densidade resposta do sistema de KohnSham. A perturbação efetiva é dada como a soma da perturbação externa do sistema 
real, do termo de Hartree linearizado e do termo xc:

$$
\begin{aligned}
v_{s 1}(\mathbf{r}, t) & =v_{1}(\mathbf{r}, t)+\int d^{3} r^{\prime} \frac{\rho_{1}\left(\mathbf{r}^{\prime}, t\right)}{\left|\mathbf{r}-\mathbf{r}^{\prime}\right|} \\
& +\int d t^{\prime} \int d^{3} r^{\prime} f_{\mathrm{xc}}\left(\mathbf{r}, t, \mathbf{r}^{\prime}, t^{\prime}\right) \rho_{1}\left(\mathbf{r}^{\prime}, t^{\prime}\right) .
\end{aligned}
$$

O chamado kernel $x c$ é definido como a partir da derivada funcional do potencial xc dependente do tempo com respeito a densidade dependente do tempo, calculada na densidade do estado fundamental:

$$
f_{\mathrm{xc}}\left(\mathbf{r}, t, \mathbf{r}^{\prime}, t^{\prime}\right)=\left.\frac{\delta v_{\mathrm{xc}}[\rho](\mathbf{r}, t)}{\delta \rho\left(\mathbf{r}^{\prime}, t^{\prime}\right)}\right|_{\rho_{0}(\mathbf{r})} .
$$

A perturbação efetiva (2.69) depende da densidade resposta, de forma que a equação resposta na TDDFT (2.68) tem de ser resolvida de forma autoconsistente. A resposta dependente da frequência é dada por

$$
\rho_{1}(\mathbf{r}, \omega)=\int d^{3} r^{\prime} \chi_{s}\left(\mathbf{r}, \mathbf{r}^{\prime}, \omega\right) v_{1 s}\left(\mathbf{r}^{\prime}, \omega\right)
$$

e

$$
\begin{aligned}
v_{s 1}(\mathbf{r}, \omega) & =v_{1}(\mathbf{r}, \omega) \\
& +\int d^{3} r^{\prime}\left\{\frac{1}{\left|\mathbf{r}-\mathbf{r}^{\prime}\right|}+f_{\mathrm{xc}}\left(\mathbf{r}, \mathbf{r}^{\prime}, \omega\right)\right\} \rho_{1}\left(\mathbf{r}^{\prime}, \omega\right)
\end{aligned}
$$

O kernel xc no domínio das frequências é a transformada de Fourier de $f_{\mathrm{xc}}\left(\mathbf{r}, t, \mathbf{r}^{\prime}, t^{\prime}\right)$ com relação a $\left(t-t^{\prime}\right)$.

A função resposta de Kohn-Sham é dada por

$$
\chi_{s}\left(\mathbf{r}, \mathbf{r}^{\prime}, \omega\right)=\sum_{j, k=1}^{\infty}\left(f_{k}-f_{j}\right) \frac{\varphi_{j}(\mathbf{r}) \varphi_{k}^{*}(\mathbf{r}) \varphi_{j}^{*}\left(\mathbf{r}^{\prime}\right) \varphi_{k}\left(\mathbf{r}^{\prime}\right)}{\omega-\omega_{j k}+i \eta}
$$

onde $f_{j}$ e $f_{k}$ são número de ocupação e se referem as configurações do estado fundamental de Kohn-Sham (1 para ocupado e 0 para orbitais de Kohn-Sham desocupados), 
e os termos $\omega_{j k}$ são definidos por

$$
\omega_{j k}=\varepsilon_{j}-\varepsilon_{k} .
$$

Portanto, $\chi_{s}\left(\mathbf{r}, \mathbf{r}^{\prime}, \omega\right)$ tem pólos nas energias de excitação do sistema de Khon-Sham.

\subsubsection{O cálculo das energias de excitação}

As energias de excitação em um sistema molecular são definidas como a diferença entre a energia do estado fundamental $E_{0}$ e a energia de um estado excitado $E_{n}$ - ver a Equação (2.67). Em outras palavras, elas são obtidas comparando as energias do estado estacionário. Então por que usar uma abordagem dinâmica como a TDDFT?

Para responder a essa pergunta, é conveniente pensar o processo de excitação de forma diferente, como um processo dinâmico onde ocorrem transições entre dois autoestados; a energia de excitação, então corresponde a uma frequência característica, que descreve o rearranjos de densidade de probabilidade, durante o processo de transição. Em outras palavras, cada excitação corresponde a um automodo do sistema de $N$ elétrons interagentes.

O conceito de automodo eletrônico possui um análogo na mecânica clássica: os modos normais de vibração de um conjunto de osciladores acoplados [153,177].

Ullrich mostra que calcular as energias de excitação com a TDDFT é muito semelhante a descrever pequenas oscilações desse sistema clássico [153]. Considere o regime de resposta linear, Equação (2.71), mas sem nenhuma perturbação externa:

$$
\rho_{1}(\mathbf{r}, \omega)=\int d^{3} r^{\prime} \chi_{s}\left(\mathbf{r}, \mathbf{r}^{\prime}, \omega\right) \int d^{3} r^{\prime \prime} f_{\mathrm{Hxc}}\left(\mathbf{r}^{\prime}, \mathbf{r}^{\prime \prime}, \omega\right) n_{1}\left(\mathbf{r}^{\prime \prime}, \omega\right)
$$

onde foi definido o kernel combinado Hartree-xc $f_{\mathrm{Hxc}}\left(\mathbf{r}, \mathbf{r}^{\prime}, \omega\right)=\left|\mathbf{r}-\mathbf{r}^{\prime}\right|^{-1}+f_{\mathrm{xc}}\left(\mathbf{r}, \mathbf{r}^{\prime}, \omega\right)$. A Equação (2.75) tem uma solução trivial $\rho_{1}=0$ para qualquer frequência $\omega$, mas certas frequências especiais $\Omega$ são soluções não triviais. Essas frequências correspondem as energias de excitação do sistema, e podem ser calculadas, em princípio exatamente, 
usando as chamadas equações de Casida [178]:

$$
\left(\begin{array}{cc}
\mathbf{A} & \mathbf{K} \\
\mathbf{K} & \mathbf{A}
\end{array}\right)\left(\begin{array}{c}
\mathbf{X} \\
\mathbf{Y}
\end{array}\right)=\Omega\left(\begin{array}{cc}
-1 & 0 \\
\mathbf{0} & \mathbf{1}
\end{array}\right)\left(\begin{array}{l}
\mathbf{X} \\
\mathbf{Y}
\end{array}\right)
$$

onde os elementos de matriz de $\mathbf{A}$ e $\mathbf{K}$ são dados por

$$
\begin{aligned}
A_{i a \sigma, i^{\prime} a^{\prime} \sigma^{\prime}}(\omega) & =\delta_{i i^{\prime}} \delta_{a a^{\prime}} \delta_{\sigma \sigma^{\prime}} \omega_{a i \sigma}+K_{i a \sigma, i^{\prime} a^{\prime} \sigma^{\prime}}(\omega) \\
K_{i a \sigma, i^{\prime} a^{\prime} \sigma^{\prime}}(\omega) & =\int d^{3} r \int d^{3} r^{\prime} \varphi_{i \sigma}^{*}(\mathbf{r}) \varphi_{a \sigma}(\mathbf{r}) \\
& \times f_{\mathrm{Hxc} \sigma \sigma^{\prime}}\left(\mathbf{r}, \mathbf{r}^{\prime}, \omega\right) \varphi_{i^{\prime} \sigma^{\prime}}\left(\mathbf{r}^{\prime}\right) \varphi_{a^{\prime} \sigma^{\prime}}^{*}\left(\mathbf{r}^{\prime}\right)
\end{aligned}
$$

$i, i^{\prime}$ e $a, a^{\prime}$ correm sobre orbitais de Kohn-Sham ocupados e desocupados, respectivamente. A dedução detalhada dessas Equações (2.76) pode se encontrada na Referência [148].

A equação 2.76 mistura excitações e desexcitações ( $\mathbf{X}$ e $\mathbf{Y}$, respectivamente). Uma forma de simplificá-la é desacoplar $\mathbf{X}$ e $\mathbf{Y}$, anulando-se os termos fora da diagonal K, Essa aproximação, conhecida como Aproximação de Tamm-Dancoff (TDA), é válida se as frequências de excitação não são próximas de zero, é bastante usada em moléculas, semicondutores e isolantes.

\subsubsection{Excitações de transferência de carga}

As excitações de transferência de carga, com já vimos na introdução, ocorrem quando a carga se move de uma região doadora (D) para outra região receptora (A) separada espacialmente da primeira. A transferência pode ser intermolecular como no caso dos CTC, ou entre diferentes grupos funcionais de uma mesma molécula (intramolecular). Infelizmente, a aproximações padrão da TDDFT falham consideravelmente na descrição de excitações de transferência de carga $[72,179,180]$.

Considere o caso onde os subsistemas $D$ e $A$ estão são separados por uma distância $R$ (muito longa). A energia mínima necessária para remover o elétron do $D$ é dada pelo seu potencial de ionização $I_{d}$. Quando o elétron é capturado pelo $A$ é liberada 
uma energia igual a sua afinidade eletrônica $A_{a}$. Uma vez que o elétron tem de se mover de $D$ para $A$ os dois sistema sentem uma interação eletrostática $-1 / R$ relativa a interação elétron-buraco. A energia de transferência de carga é portanto

$$
\Omega_{c t}^{\text {exact }}=I_{d}-A_{a}-\frac{1}{R}
$$

Agora vamos comparar com a TDDFT. Para isso é suficiente considerar um sistema de dois níveis, para o qual:

$$
\begin{aligned}
\Omega_{c t}= & \varepsilon_{L}^{a}-\varepsilon_{H}^{d}+2 \int d^{3} r \int d^{3} r^{\prime} \varphi_{L}^{a}(\mathbf{r}) \varphi_{H}^{d}(\mathbf{r}) \\
& \times f_{\mathrm{Hxc}}\left(\mathbf{r}, \mathbf{r}^{\prime}, \omega\right) \varphi_{L}^{a}\left(\mathbf{r}^{\prime}\right) \varphi_{H}^{d}\left(\mathbf{r}^{\prime}\right)
\end{aligned}
$$

onde $\varphi_{H}^{d}(\mathbf{r})$ é o orbital mais alto ocupado de $D$ e $\varphi_{L}^{a}(\mathbf{r})$ é o orbital mais baixo desocupado em $A$, que tem um overlap que se anula no limite de grande separação de carga. Daí a integral dupla na Equação (2.80) se anula (assumindo que o kernel xc kernel permanece finito, o que é o caso para todas as aproximações padrão), e a TDDFT simplesmente descreve a diferença entre os autovalores de Kohn-Sham,

$$
\Omega_{c t} \longrightarrow \varepsilon_{L}^{a}-\varepsilon_{H}^{d}
$$

Isso explica o porquê da TDDFT subestima dramaticamente a energia de excitação de transferência de carga quando funcionais xc convencionais são usados. Funcionais xc híbridos [181,182], em particular os híbridos com correção de longo alcance (rangeseparated hybrids) vistos na Seção 2.3.4, oferecem uma solução a esse problema, e tem sido usados com êxito na descrição de sistemas com excitações de transferência de carga $[77,183,184]$. Dentre estes, destacam-se os funcionais CAM-B3LYP e o funcional $\omega$ B97X-D $[73,185,186]$ que serão usados neste trabalho para o cálculo das energias de excitação dos CTC. 


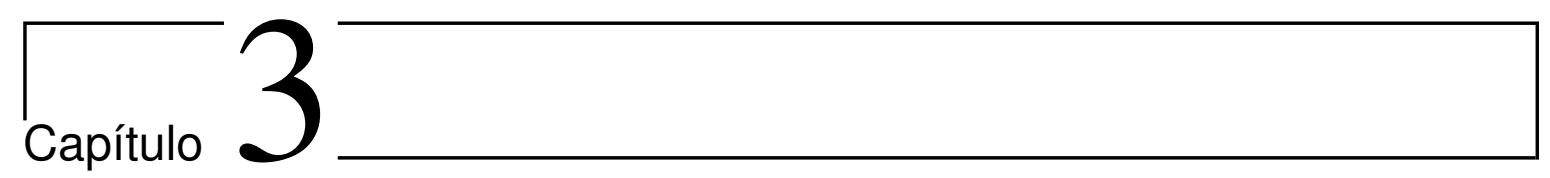

\section{Dinâmica molecular}

Neste capitulos apresentamos em maiores detalhes as técnicas de dinâmica molecular clássica e dinâmica molecular de Born-Oppenheimer. No final do capítulo apresentamos algumas técnicas usadas posteriormente para análise da dinâmica molecular. 


\subsection{Introdução}

Vimos no capítulo anterior que encontrar uma solução para o problema molecular é uma tarefa bastante árdua, sendo necessário realizar uma série de aproximações. Após o uso da aproximação de Born-Oppenheimer, nos concentramos em encontrar soluções para o problema eletrônico, e todo o desenvolvimento posterior da DFT e TDFT foi realizado supondo uma estrutura rígida para os núcleos. Essa hipótese possui sérias limitações quando se estuda moléculas em solução pois nessas condições as moléculas e macromoléculas em geral não são rígidas, mas sofrem rearranjos em suas estruturas ocasionadas por diversos efeitos como flutuações térmicas e difusão ou em resposta a perturbações externas como pressão, concentração e mudanças de temperatura.

O comportamento microscópico do sistema em geral não é acessível experimentalmente e os métodos experimentais usados na elucidação estrutural possuem limitações. Nos últimos anos os métodos de dinâmica molecular, seja por primeiros princípios ou baseada em campos de força clássicos, têm se tornado uma ferramenta importante no auxílio da compreensão do comportamento de sistemas no nível microscópico.

\subsection{Aproximação clássica}

Vimos no capítulo anterior (ver seção 2.2) que, usando a aproximação de BornOppenheimer, é possível separar os movimentos nuclear e eletrônico. Um desenvolvimento semelhante para a equação de movimento quântica,

$$
\mathcal{H} \Psi(\mathbf{x}, \mathbf{R} ; t)=-\frac{\partial}{\partial t} \Psi(\mathbf{x}, \mathbf{R} ; t)
$$

permite chegar a uma equação apenas para os núcleos (uma dedução pode ser encontrada na referência [187]),

$$
\left[\hat{T}_{N}+V_{N N}(\mathbf{R})+E(\mathbf{R})\right] \Phi_{N}(\mathbf{R})=i \frac{\partial}{\partial t} \Phi_{N}(\mathbf{R})
$$

onde $E(\mathbf{R})$ são autovalores do problema eletrônico estacionário 2.4. Para os sistemas na fase condensada, uma descrição realista exige o tratamento de um número grande 
de graus de liberdade nucleares, tornando a busca pela solução do problema (3.2) uma tarefa computacionalmente impraticável.

Felizmente, com exceção de alguns sistemas anômalos, como o hélio líquido, grande parte dos sistemas na fase condensada podem ser tratados usando a aproximação clássica para a equação de movimento (ver, por exemplo, [188] e o capítulo 12 da referência [189]). De fato, é possível mostrar que a Equação (3.2) tem como limite as equações clássicas de movimento (ver [187]).

\subsection{Dinâmica molecular de Born-Oppenheimer}

A dinâmica molecular de Born-Oppenheimer (BOMD) é uma abordagem que permite incluir a estrutura eletrônica na dinâmica nuclear, resolvendo a cada passo a equação de Schödinger eletrônica para uma configuração nuclear fixa em um dado instante de tempo. Portanto, o problema eletrônico é resolvido a cada passo, definindo um potencial que influencia o movimento nuclear, cujas posições são propagadas no tempo usando as leis da dinâmica clássica. O método pode ser definido usando as seguintes equações

$$
\begin{gathered}
M_{I} \ddot{\mathbf{R}}_{I}(t)=-\nabla_{I} \min _{\Psi_{0}}\left\{\left\langle\Psi_{0}|\hat{H}| \Psi_{0}\right\rangle\right\}, \\
\hat{H} \Psi_{0}=E_{0} \Psi_{0},
\end{gathered}
$$

onde $\hat{H}$ é o Hamiltoniano eletrônico (2.6), $E_{0}$ e $\Psi_{0}$ referem-se ao estado fundamental.

A idéia do método é bastante simples, mas o custo computacional necessário para a solução do problema eletrônico agora é multiplicado pelo número de passos da dinâmica, uma vez que a cada passo um cálculo quântico da energia do sistema tem de ser realizado. A grande vantagem do método é que o potencial de interação é calculado de forma bastante acurada. Mas o alto custo computacional limita a aplicação desse tipo de dinâmica a escala de poucos picossegundos, além de um sistema da ordem de centenas de átomos. A fim de reduzir o custo computacional desse tipo dinâmica, uma implementação eficiente para a solução do problema eletrônico é essencial. Neste trabalho usamos uma implementação bastante sofisticada do método, encontrada no módulo QuickStep [190] do programa de estrutura eletrônica CP2K [191]. 
A implementação da DFT usa um procedimento híbrido para o cálculo da energia por meio das equações de Kohn-Sham: o método GPW.

\subsubsection{O método GPW}

A idéia central do método GPW [192] (do inglês "Gaussian and Plane Waves") consiste em usar duas representações para a densidade eletrônica. Além das funções bases gaussianas, usadas para representar as funções de onda, um conjunto de ondas planas auxiliar é usado para descrever a densidade. Com esta base auxiliar de ondas planas, o termo de Hartree pode ser calculado a um custo que cresce linearmente com o tamanho do sistema.

A primeira representação da densidade eletrônica é baseada em funções de onda gaussianas

$$
\rho(\mathbf{r})=\sum_{\mu \nu} P^{\mu \nu} \phi_{\mu}(\mathbf{r}) \phi_{\nu}(\mathbf{r})
$$

onde $P$ é um elemento de matriz densidade, e $\varphi$ é a expansão

$$
\varphi=\sum_{i} d_{i \mu} g_{i}(\mathbf{r})
$$

onde $d_{\mu \nu}$ correspondem aos coeficientes das primitivas gaussianas $g_{i}(\mathbf{r})$.

A segunda representação usa uma base auxiliar de ondas planas, e é dada por

$$
\tilde{\rho}(\mathbf{r})=\frac{1}{\Omega} \sum_{G} \tilde{\rho}(G) \exp (i G \cdot \mathbf{r})
$$

onde $\Omega$ é o volume da célula unitária, e $\mathbf{G}$ são vetores da rede no espaço recíproco. O coeficientes $\tilde{\rho}(\mathbf{r})$ são escolhidos de tal forma que $\tilde{\rho}(\mathbf{r})$ seja igual a $\rho(\mathbf{r})$ em um grid regular definido na célula unitária. A diferença $|\tilde{\rho}(\mathbf{r})-\rho(\mathbf{r})|$ vai a zero quando a energia de corte $E_{\text {cut }}$ tende a infinito [192]. 


\subsubsection{Pseudopotenciais de GTH}

Normalmente os elétrons de caroço não têm grande influência sobre as propriedades eletrônicas, e a maior parte das propriedades físico-químicas estão associadas principalmente aos elétrons de valência. Isso significa que uma descrição acurada dos elétrons de caroço não é necessária em muitas aplicações práticas. É possível tomar vantagem desse fato e simplificar a descrição dos átomos de um sistema molecular usando um pseudopotencial para descrever os elétrons de caroço.

A idéia do pseudopotencial é descrever explicitamente apenas os elétrons de valência e substituir os elétrons de caroço por um potencial modificado. Esse potencial deve reproduzir o mesmo comportamento caso todos os elétrons do sistema fossem tratados explicitamente.

Para alcançar tal objetivo, os pseudopotenciais em geral são construídos respeitando certas condições como, por exemplo, reproduzir a função de onda dos elétrons explícitos para valores além do raio de corte $r_{\text {core }}$ ) [193]. Além disso devem obedecer a condição de norma conservada ("norm conserving") [194],

$$
\int_{|\mathbf{r}|<R}\left|\Psi_{\text {pseudo }}(\mathbf{r})\right|^{2} \mathrm{~d} \mathbf{r}=\int_{|\mathbf{r}|<R}\left|\Psi_{\text {all-electron }}(\mathbf{r})\right|^{2} \mathrm{~d} \mathbf{r}
$$

para todo $R>r_{\text {core }}$.

Neste trabalho usamos os pseudopotenciais propostos por Goedecker, Teter e Hutter (GTH) [195, 196]. É um pseudopotencial cuja forma analítica que possibilita uma implementação eficiente dentro do formalismo GPW. Ele constituído por um termo local que por sua vez possui um termo de longo alcance (LR, "long-ranged") e um termo de curto alcance (SR, "short-ranged")

$$
\begin{aligned}
V_{l o c}^{P P}(r) & =V_{l o c}^{L R}(r)+V_{l o c}^{S R}(r) \\
& =-\frac{Z}{r} \operatorname{erf}\left(\alpha^{P P} r\right)+\sum_{i=1}^{4} C_{i}^{P P}\left(\sqrt{2} \alpha^{P P} r\right)^{2 i-2} \exp \left[-\left(\alpha^{P P} r\right)^{2}\right]
\end{aligned}
$$

com

$$
\alpha^{P P}=\frac{1}{\sqrt{2} r_{l o c}^{P P}} .
$$


O pseudopotencial é constituído ainda por um termo não local, dado por

$$
V_{n l}^{P P}\left(\mathbf{r}, \mathbf{r}^{\prime}\right)=\sum_{l m} \sum_{i j}\left\langle\mathbf{r} \mid p_{i}^{l m}\right\rangle h_{i j}^{l}\left\langle p_{j}^{l m} \mid \mathbf{r}^{\prime}\right\rangle
$$

onde

$$
\left\langle\mathbf{r} \mid p_{i}^{l m}\right\rangle=N_{i}^{l} Y^{l m}(\hat{r}) r^{l+2 i-2} \exp \left[-\frac{1}{2}\left(\frac{r}{r_{l}}\right)^{2}\right],
$$

$N_{i}^{l}$ são constantes de normalização e $Y^{l m}(\hat{r})$ são harmônicos esféricos.

Os parâmetros $\left(r_{l o c}^{P P}, C_{i}^{P P}, r_{l}\right.$ e $\left.h_{i j}^{l}\right)$ do pseudopotencial de GTH são otimizados para reproduzir a função de onda obtida com cálculos de DFT usando inclusive correções relativísticas. Esse pseudopotencial consegue, portanto, descrever até mesmo elementos pesados com acurácia. Mais detalhes sobre a implementação do método pode ser encontrados na referência [190].

\subsection{Dinâmica molecular clássica}

A Dinâmica Molecular clássica ou simplesmente MD ("Molecular Dynamics") também busca a solução numérica, das equações de movimento para um sistema de átomos ou moléculas interagentes, cujas equações de movimento assumem a forma

$$
m_{i} \frac{\partial^{2} \mathbf{r}_{i}}{\partial t^{2}}=\mathbf{F}_{i}, i=1, \ldots, N ; \mathbf{F}_{i}=\frac{\partial U}{\partial \mathbf{r}_{i}}
$$

onde as forças são de caráter conservativas e derivam de um potencial

$$
U\left(\mathbf{r}^{N}=\left(\mathbf{r}_{1}, \ldots, \mathbf{r}_{N}\right)\right) .
$$

A dinâmica molecular clássica torna-se atrativa para sistemas com muitos átomos pois usa uma forma funcional aproximada para a energia potencial $U(\mathbf{r})$, que visa reproduzir propriedades termodinâmicas ou representar no contexto da mecânica clássica o potencial de origem quântica. Em geral são escritos na forma de uma soma de um potencial intermolecular (ou potencial não ligado) e intramolecular (ou potencial ligado). 
A parte intermolecular descreve interações entre os átomos de moléculas diferentes (e também interações entre os átomos mais distantes de uma mesma molécula, separados além do $3^{o}$ vizinho). Já o termo intramolecular descreve os graus de liberdade internos da molécula (rotações, vibrações e torções). Os conjuntos de parâmetros necessários para a descrição dessas interações são conhecidos na literatura como campos de força. Os campos de força são obtidos por meio de ajustes a dados experimentais ou ainda por cálculos quânticos. Diversos grupos se dedicam exclusivamente a arte de obter esses conjuntos de parâmetro. Alguns conjuntos de notoriedade são o OPLS (Optimized Parameter for Liquid Simulation) [197], AMBER (Assisted Model Building with Energy Refinement) [198,199], GROMOS(GROningen MOlecular Simulation) [200-203], CHARMM (Chemistry at HARvard Macromolecular Mechanics) [204-206], etc.

Neste trabalho usamos o OPLS, pois esse campo de força possui parâmetros para um número grande de sistemas semelhantes ao que estudamos: piridinas, cadeias carbônicas, solventes orgânicos e íons halogênios [197, 207-209].

Na maior parte dos campos de força, as interações moleculares são modeladas por um potencial efetivo de dois corpos,

$$
U\left(\mathbf{r}_{1}, \ldots, \mathbf{r}_{N}\right)=\sum_{i<j} V_{i j}\left(\mathbf{r}_{i}, \mathbf{r}_{j}\right)
$$

onde normalmente assume-se que as interações dependem apenas da distância relativa entre os átomos ou sítios de interação, ou seja,

$$
V_{i j}\left(\mathbf{r}_{i}, \mathbf{r}_{j}\right)=V_{i j}\left(r_{i j}\right)
$$

Para líquidos a forma mais comum de descrever as interações entre átomos não ligados é o potencial de Lennard-Jones (LJ) acrescido do termo de Coulomb (C),

$$
V_{L J}\left(r_{i j}\right)=4 \varepsilon_{i j}\left(\left(\frac{\sigma_{i j}}{r_{i j}}\right)^{12}-\left(\frac{\sigma_{i j}}{r_{i j}}\right)^{6}\right) .
$$

O termo atrativo do potencial de LJ, proporcional a $r^{-6}$, visa reproduzir as forças de dispersão van der Waals. A curta distância, o potencial de LJ é dominado pelo 
termo $r^{-12}$, e modela a repulsão eletrônica dos átomos. O expoente 12 , ao contrário do termo atrativo, não tem origem física e é escolhido por razões práticas, a fim de facilitar o cálculo, já que é quadrado do termo atrativo. Cada sítio do potencial de LJ é descrito por dois parâmetros: o parâmetro $\varepsilon_{i j}$ é a profundidade do poço de potencial, modelando a força da interação e o parâmetro $\sigma_{i j}$, que se relaciona ao tamanho do sistema, é a distância finita onde o potencial se anula, (a distância do mínimo do potencial é de $1.222 \sigma_{i j}$ ). Os termos mistos são obtidos por meio de uma regra de combinação geométrica,

$$
\sigma_{i j}=\left(\sigma_{i} \cdot \sigma_{j}\right)^{1 / 2} ; \quad \varepsilon_{i j}=\left(\varepsilon_{i} \cdot \varepsilon_{j}\right)^{1 / 2}
$$

Já as cargas para o potencial de Coulomb descrevem as interações eletrostáticas entre as moléculas,

$$
V_{C}\left(r_{i j}\right)=\frac{q_{i} q_{j}}{r_{i j}}
$$

As interações entre átomos ligados ( $1^{\circ}$ ao $3^{\circ}$ vizinho), descrevem os graus internos de liberdade de uma molécula (deformações moleculares) e são compostos pela soma de vários termos,

$$
U_{\text {lig }}=\sum_{\text {ligações }} V_{b}\left(r_{i j}\right)+\sum_{\text {ângulos }} V_{a}\left(\theta_{i j k}\right)+\sum_{\text {diedros }} V_{d}\left(\phi_{i j k l}\right)+\sum_{\text {impróprios }} V_{i}\left(\phi_{i j k l}\right) .
$$

onde $r_{i j}$ correspondem às distâncias de ligação, $\theta_{j k l}$ correspondem aos ângulos de ligação e $\phi_{i j k l}$ aos ângulos de torção. Estes conceitos são ilustrados na Figura 3.1.

Na equação 3.20 acima, o estiramento entre dois átomos ligados covalentemente, responsáveis pelo movimento de vibração, é representado tipicamente por meio de um potencial harmônico composto por dois parâmetros: a constante de mola $k_{i j}^{b}$ e posição de equilíbrio $b_{i j}$.

A mesma aproximação é tipicamente usada na descrição das vibrações angulares, cujos parâmetros para a constante de mola e o ângulo equilíbrio são $k_{i j k}^{a}$ e $\theta_{i j k}$, respectivamente.

As formas funcionais mais comuns para o potencial de estiramento de ligação e 


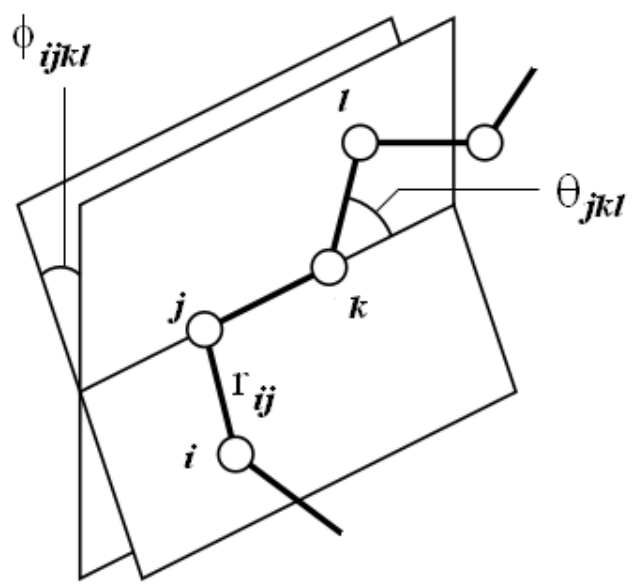

Figura 3.1: Representação de uma cadeia molecular de átomos, ilustrando os conceitos de distância ligação $r_{i j}$, ângulo $\theta_{j k l}$ e ângulo de torção $\phi_{i j k l}$ (ou ângulo diedral). Adaptada da Referência [93]

deformações angulares são mostradas nas equações 3.21,

$$
\begin{gathered}
V_{b}\left(r_{i j}\right)=\frac{1}{2} k_{i j}^{b}\left(r_{i j}-b_{i j}\right)^{2}, r_{i j}=\left|\mathbf{r}_{i}-\mathbf{r}_{j}\right| \\
V_{a}\left(\theta_{i j k}\right)=\frac{1}{2} k_{i j k}^{\theta}\left(\theta_{i j k}-\theta_{i j k}^{0}\right), \cos \theta=\frac{\mathbf{r}_{i j} \cdot \mathbf{r}_{k l}}{r_{i j} r_{k l}}
\end{gathered}
$$

A forma funcional mais comum para o potencial de torção é mostrada na equação 3.22. O potencial é periódico e é capaz de descrever as diferentes conformações moleculares: cis, trans e gauche, etc.

$$
V_{d}\left(\phi_{i j k l}\right)=\sum_{m} k_{i j k l}^{\phi, m}\left(1+\cos \left(m \phi_{i j k l}-\phi_{m}\right)\right)
$$

Finalmente, diedros impróprios também são definidos a fim de garantir a forma plana (em anéis aromáticos, por exemplo) ou tetraédrica de certos grupos.

Os parâmetros do campo de força relativos ao sistema molecular de interesse são guardados em um arquivo de topologia. Cada campo de força possui parâmetros independentes, que não devem ser misturados entre si. Alguns campos de força são de- 
senhados para descrever um determinado tipo de sistema, como proteínas (compostas por aminoácidos), açúcares ou moléculas orgânicas em geral, como é o caso do OPLS.

Os campos de força são construídos decompondo as interações moleculares em interações entre os átomos ou grupos atômicos. Assim, ainda que um sistema não tenha ainda sido parametrizado por completo, é possível descrevê-lo utilizando parâmetros de sistemas similares que já tenham sido parametrizados, usando os parâmetros dos grupos em comum.

Esse princípio é conhecido como transferabilidade dos parâmetros. Na prática os constituintes do sistema de interesse são agrupados em grupos atômicos que já possuam parâmetros no campo de força. Os parâmetros restantes podem ser ajustados por meio de cálculos quânticos, por exemplo.

A topologia dos derivados de piridínio de interesse neste trabalho foi construída usando os parâmetros do campo de força OPLS para piridina, alcanos e halogênios [197,207-210]. Existem alguns trabalhos na literatura que usaram estes parâmetros para estudar a dinâmica de líquidos iônicos formados por derivados de piridínio [44-50].

Mas como neste trabalho o objetivo é o estudo dos complexos em acetonitrila, algumas modificações no campo de força são necessárias. A principal modificação são as cargas usadas no potencial de Coulomb, que podem variar bastante com o meio no qual o soluto é inserido $[123,125,141]$. Nesse caso, é preciso obter cargas que descrevam o sistema de forma mais adequada. Em nosso caso, as cargas foram ajustadas usando cálculos quânticos da densidade eletrônica de carga e ajustando em seguida o potencial eletrostático do sistema usando a metodologia CHELPG [211]. Nesse ajuste, um cálculo quântico é realizado para determinar a distribuição de carga do sistema. O potencial eletrostático gerado pela distribuição eletrônica é mapeado em uma rede de pontos gerado ao redor do sistema de interesse. Finalmente, cargas atômicas pontuais são ajustadas sobre os sítios atômicos, de forma que o campo eletrostático gerado por essas cargas reproduza mapa eletrostático na rede. Maiores detalhes do refinamento do campo de força serão apresentados posteriormente no capítulo de resultados. 


\subsubsection{Condições periódicas de contorno}

A dinâmica molecular é normalmente implementada assumindo um sistema de $N$ átomos em uma caixa cúbica (como é o nosso caso) de largura $L$ e volume $V=L^{3}$. O valor de $N$ atualmente é tipicamente da ordem de $10^{3}-10^{6}$, muito longe do limite termodinâmico. Assim o número relativo de partículas na superfície do sistema é muito maior em uma dinâmica molecular do que em um sistema macroscópico (onde o número de átomos é da ordem do número de Avogadro). Para minimizar os efeitos da superfície a caixa de simulação é replicada infinitamente em todas as direções, adotando-se condições periódicas de contorno (PBC). Na prática são consideradas explicitamente apenas $N$ moléculas e assim que uma delas sai da caixa por qualquer um dos lados, sua imagem entra pelo lado oposto de forma que o número total de moléculas é conservado.

Como grande a maior parte do custo computacional na MD está associado ao cálculo das forças, as PBC são usadas em conjunto com à convenção de mínima imagem, o que significa que cada partícula é colocada no centro da caixa e interage apenas com partículas (ou imagens) que estão dentro da caixa, evitando a interação da partícula com a sua imagem ou com duas vezes com a mesma molécula [87]. Assim a distância máxima de interação é $L / 2$.

Na prática usa-se um raio de corte $r_{c} \leq L / 2$, além do qual as interações entre as partículas é substituída por uma forma aproximada. As interações relativas ao potencial de Lennard-Jones sofrem um decaimento bastante acentuado e de forma geral a contribuição de curto alcance (dentro do raio de corte) é suficiente, e as contribuições de longo alcance (além do raio de corte) podem ser desprezadas sem que haja prejuízos para a energia de interação.

As interações entre cargas pontuais descritas pelo potencial Coulombiano, no entanto, decaem mais lentamente com a distância, e correções de longo alcance são necessárias para tratar as interações eletrostáticas de forma adequada. Dois métodos são amplamente usados para esse tipo de correção: o campo de reação e somas de Ewald. 


\subsubsection{Correções de longo alcance para a interação eletrostá- tica}

No método campo de reação [212,213] a vizinhança de uma partícula é separada em duas regiões. Uma cavidade esférica de raio $\mathrm{R}$ (igual ao raio de corte), preenchida com cargas pontuais é aberta em um meio contínuo de constante dielétrica $\varepsilon_{r f}$ igual à constante dielétrica do solvente de interesse. Aplica-se a condição de continuidade na fronteira

$$
V^{C b R f}\left(r_{i}, r_{j}\right)=\theta\left(R-r_{i j}\right) \frac{q_{i} q_{j}}{4 \pi \varepsilon_{0}}\left(\frac{1}{r_{i j}}-\frac{\alpha r_{i j}^{2}}{2 R^{3}}-\frac{1-\alpha / 2}{R}\right)
$$

onde

$$
\alpha=\frac{2\left(\varepsilon_{r f}-1\right)}{\left(1+\varepsilon_{r f}\right)}
$$

e $\theta(x)$ é a função de passo de Heavside. O método de campo de reação é bastante atrativo do ponto de vista computacional, $\mathcal{O}\left(R^{3} N\right)$, além de ser facilmente implementado. Entretanto, efeitos artificiais podem aparecer em sistemas muito carregados, sobretudo se a distâncias entre as partículas carregadas forem próximas ao raio de corte.

Uma forma mais rigorosa de tratar a interação eletrostática seria considerar explicitamente a entre todas as caixas replicadas,

$$
U=\frac{1}{2} \sum_{\mathbf{n}} \sum_{i=1}^{N} \sum_{j=1}^{N} \frac{Z_{i} Z_{j}}{\left|\mathbf{r}_{i j}+\mathbf{n} L\right|}
$$

onde a soma sobre $\mathbf{n}=\left(n_{x}, n_{y}, n_{z}\right)$ inclui todas as imagens periódicas e $i \neq j$ para $\mathbf{n}=\mathbf{0}$.

O grande problema é que a série (3.25) é apenas condicionalmente convergente, o que torna o processo bastante delicado e ineficiente do ponto de vista computacional. Ewald propôs uma forma eficiente de lidar com esse tipo de problema. A idéia básica das somas de Ewald é decompor a série em duas contribuições: de curto e de longo alcance. A contribuição de curto alcance converge rapidamente e é tratada diretamente no espaço real. Já a contribuição de longo alcance é a parte lentamente convergente, mas que pode ser tratada eficientemente no espaço recíproco usando transformadas de Fourier. A visão detalhada do método pode ser encontrada na referência [87].

Apesar de ser a forma mais adequada para o tratamento da interação Coulom- 
biana, o custo computacional do método cresce com $\mathcal{O}\left(N^{3 / 2}\right)$ no número de átomos do sistema. Embora seja muito mais rápido que a soma original, o algoritmo tornase ineficiente conforme o tamanho do sistema aumenta. Alguns algoritmos baseados nas somas de Ewald conseguem reduzir o custo computacional para uma eficiência $\mathcal{O}(N \log N)$, dentre eles o PME ("Particle-Mesh Ewald”) [214,215] - Para uma revisão desses métodos, ver referência [216].

Neste trabalho usamos o método PME tal qual implementado no programa GROMACS [217-222].

\subsection{Integração das equações de movimento}

Uma vez especificado o potencial, seja de origem quântica ou através de um campo de força clássico, resta escolher um algoritmo para o cálculo das forças e solução das equações de movimento. O método de diferenças finitas é a escolha padrão dos algoritmos de solução das equações de Newton. Todos os algoritmos de integração assumem que posição, velocidade e aceleração podem ser expandidas em uma série de potências. Neste trabalho usamos o algoritmo conhecido como leap-frog [223]. Ele usa a posição em $\mathbf{r}(t)$ e velocidade em $\mathbf{v}(t-(1 / 2) \delta t)$ para calcular a força. Posições e velocidades são atualizadas usando a força $F(t)$ calculada a partir da posição no instante t.

$$
\begin{aligned}
\mathbf{v}\left(t+\frac{1}{2} \delta t\right) & =\mathbf{v}\left(t-\frac{1}{2} \delta t\right)+\frac{\delta t}{m} \mathbf{F}(t) \\
\mathbf{r}(t+\delta t) & =\mathbf{r}(\delta t)+\delta t \mathbf{v}\left(t+\frac{1}{2} \delta t\right)
\end{aligned}
$$

\subsection{Dinâmica molecular e mecânica estatística}

A dinâmica molecular gera configurações microscópicas (posições e velocidades) acessíveis ao sistema de interesse. A conexão com as grandezas macroscópicas como pressão, volume, capacidade térmica, etc, é realizada usando a mecânica estatística. Considere um sistema de $N$ partículas descrito por uma função Hamiltoniana da forma

$$
H(\mathbf{r}, \mathbf{p})=T(\mathbf{r}, \mathbf{p})+U(\mathbf{r}, \mathbf{p}),
$$


onde $\mathbf{r}, \mathbf{p}$ representam conjuntamente as variáveis de posição e momento das partículas, respectivamente.

A evolução temporal do sistema é dada pelas equações de Hamilton,

$$
\dot{r}_{i}=\frac{\partial H}{\partial p_{i}} \quad \dot{p}_{i}=\frac{\partial H}{\partial r_{i}},
$$

O valor de um observável $A$ é calculado como a média temporal definida como sendo igual a

$$
\bar{A}=\lim _{\tau \rightarrow \infty} \frac{1}{\tau} \int_{0}^{\tau} d t A[\mathbf{r}(t), \mathbf{p}(t)] .
$$

A mecânica estatística está baseada na hipótese ergódica de que a média temporal é equilvalente e a média sobre o ensemble

$$
\bar{A}=\langle A\rangle_{\text {ensemble }} .
$$

Como os algoritmos de integração usam intervalos de tempo $\Delta t$ de forma que $t \rightarrow t_{k} \Delta t$ a média temporal é realizada sobre um intervalo de tempo finito $\tau=n \Delta t$

$$
(\bar{A})_{M D}=\frac{1}{n} \sum_{k=1}^{n} A\left[\mathbf{r}\left(t_{k}\right), \mathbf{p}\left(t_{k}\right)\right]
$$

Como as forças são conservativas a energia $E=K+U$ é uma constante de movimento. Do ponto de vista da mecânica estatística a MD amostra configurações do ensemble microcanônico NVE (onde se conserva o número de partículas $\mathrm{N}$, o volume $\mathrm{V}$ e a energia E do sistema).

Diversos algoritmos, conhecidos como termostatos, foram criados com o objetivo de amostrar configurações em um ensemble termodinâmico de temperatura constante (NVT). O termostato de Berendsen acopla o sistema a um reservatório térmico externo de temperatura $T_{0}$. Desvios da temperatura são corrigidos usando uma equação diferencial de primeira ordem,

$$
\frac{d T}{d t}=\frac{T_{0}-T}{\tau}
$$

o que significa que o desvio de temperatura decai exponencialmente com uma taxa de decaimento $\tau$. Embora seja um método robusto e bastante usado, esse algoritmo afeta 
as flutuações na energia cinética, resultando em um ensemble que a rigor não corresponde ao ensemble canônico. Neste trabalho usamos um termostato estocástico de escalonamento de velocidades [224], que é baseado no de Berendsen, mas que consegue amostrar o ensemble corretamente. O acoplamento é realizado usando uma equação diferencial semelhante a equação (3.32), com um termo estocástico adicional capaz de assegurar a flutuação correta da energia cinética:

$$
d K=\left(K_{0}-K\right) \frac{d t}{\tau_{T}}+2 \sqrt{\frac{K K_{0}}{N_{f}}} \frac{d W}{\tau_{T}}
$$

onde $K$ é a energia cinética do sistema, $d W$ é um termo estocástico e $N_{f}$ é o número de graus de liberdade do sistema. Mais detalhes sobre esse algoritmo e a comparação com os outros termostatos podem ser encontrados nas referências [224,225].

Além da temperatura, o sistema pode ser acoplado a barostatos para se gerar configurações em um ensemble onde a pressão P também é constante (NPT). Neste trabalho usamos o barostato de Berendsen, que modifica as coordenadas e o tamanho da caixa a um determinado número $n_{P} C$ de passos.

\subsection{O protocolo de simulação}

A solução das equações de movimento necessita de uma configuração inicial. Em princípio essa condição poderia ser gerada arbitrariamente, já que não influenciaria o equilíbrio. Na prática é conveniente escolher adequadamente uma condição inicial, que evite por exemplo o contato muito próximo dos átomos, o que levaria ao surgimento de forças repulsivas muito fortes, que poderiam colapsar o sistema. Uma maneira comum é iniciar a dinâmica colocando-se os átomos sobre uma rede cristalina. Em nosso caso, foi usada como condição inicial para o soluto a geometria de equilíbrio do cálculo de mecânica quântica. O solvente é introduzido no sistema a partir de uma caixa contendo 500 moléculas de acetonitrila pré equilibradas. Um algoritmo é usado para replicar esta caixa de solvente ao redor do soluto, que é centralizado na caixa de simulação. As moléculas de solvente que se sobrepõe ao soluto são então excluídas. Antes de iniciar a dinâmica clássica, é aconselhável realizar no sistema uma minimização de energia no campo de força clássico, eliminando qualquer força espúria que ainda exista no 
sistema. Um dos métodos mais simples e robustos para encontrar a configuração de mínima energia é o Steepest Descent. Nesse método, usa-se a derivada primeira para encontrar a direção do mínimo de energia.

Após a minimização, inicia-se o processo de equilíbrio ou termalização, que consiste em deixar o sistema evoluir até atingir o equilíbrio termodinâmico. O tempo de simulação para que esse processo ocorra depende do sistema de estudo e não existe uma forma de determiná-lo. Na prática deixa-se o sistema evoluir até a que grandezas como energia, temperatura, pressão, etc. convirjam e comecem a oscilar em torno de um valor médio de equilíbrio. Para moléculas em solução, como os estudados neste trabalho, este tempo foi tipicamente da ordem de picossegundos. Após o estagio de equilíbrio, inicia-se a fase de produção. Nessa etapa informações sobre a trajetória e médias de algumas propriedades são guardadas em arquivo para posterior análises.

\subsection{Procedimentos de análise}

Uma vez finalizada a dinâmica molecular, as coordenadas, velocidades, além de grandezas como energia, temperatura, pressão, etc. estão guardadas em arquivos. O próximo passo consiste na análise destes resultados.

O processo de análise pode ser separado em dois estágios. O primeiro estágio consiste em algumas análises preliminares à procura de possíveis erros ou inconsistências. Nesta etapa analisam-se a evolução e convergência de propriedades termodinâmicas como energia potencial, temperatura, pressão. Verifica-se a flutuação ao redor do valor médio e se os mesmos são compatíveis com valores de referência, valores experimentais ou pré-definidos no pelos termostatos e barostatos. No caso particular do ensemble NPT uma grandeza bastante usada para aferir a qualidade da dinâmica de líquidos é a densidade, uma vez que ela pode ser comparada diretamente com o valor experimental.

Após a checagem, passa-se ao segundo estágio onde se analisam as propriedades de interesse. Propriedades termodinâmicas usuais como energia, entalpia e volume podem ser obtidas a partir da análise da dinâmica molecular, sendo expressas como médias configuracionais. Também podem ser obtidas propriedades relacionadas às flutuações estatísticas destas propriedades, como capacidade calorífica, coeficientes de 
expansão térmica e de compressão isobárica. Análises estruturais como comprimentos de ligação, ângulos, diedros também podem ser realizadas a partir das informações contidas no arquivo de trajetória. Dentre outras análises estruturais, vale a pena ressaltar:

\subsubsection{RMSD}

O RMSD mede a diferença entre uma dada conformação e uma estrutura de referência,

$$
R M S D=\frac{1}{N}\left(\sum_{i j}^{N}\left(\mathbf{r}_{i j}^{r e f}-\mathbf{r}_{i j}\right)^{2}\right)^{1 / 2}
$$

onde $\mathbf{r}_{i j}^{\text {ref }}$ denotam as coordenadas da estrutura de referência. Ao longo da trajetória as moléculas sofrem não só mudanças conformacionais (comprimento de ligações, ângulos e diedros) como também movimentos de rotação e translação.

No cálculo do RMSD, cada estrutura é inicialmente superposta a estrutura inicial usando operações de rotação e translação. Em seguida calcula-se o desvio quadrático médio das posições, de modo a obter o menor RMSD. Este também poder avaliado ao longo da trajetória em relação á estrutura inicial, a fim de se avaliar as mudanças conformacionais ao longo da dinâmica. Neste trabalho o RMSD ao longo da trajetória foi calculado usando a ferramenta "g_rms" implementada no programa GROMACS.

\subsubsection{Distribuição radial de pares}

A função $G(r)$ de distribuição radial de pares (RDF) permite obter características da estrutura do líquido. Por estrutura entende-se a disposição média relativa entre as moléculas. Vale ressaltar que esta informação pode ser obtida experimentalmente por técnicas de espalhamento de raios-X e difração de nêutrons.

A informação da RDF é obtida da simulação calculando o histograma de distância entre os pares $i$ e $j$,

$$
G(r)=\frac{n_{i j}(r, r+d r)}{n_{i d}(r, r+d r)}
$$

onde $n_{i j}$ é o número de pares $i j$ separados por uma distância entre $r$ e $r+d r, n_{i d}$ é o 
número de pares no gás ideal de mesma densidade,

$$
n_{i d}=\frac{4 \pi}{3} \rho\left[(r+d r)^{3}-r^{3}\right]
$$

A integração de $G_{i j}(r)$ em até uma distância $r$,

$$
N_{s}=\frac{4 \pi N}{V} \int_{0}^{r} G_{i j}(r) r^{2} d r
$$

dá informação sobre o número de moléculas $N_{s}$ que se distribuem radialmente ao redor de outra. O número de moléculas no primeiro pico é conhecido como número de coordenação e é usado para definir a primeira camada de solvatação. Demais camadas também podem ser associadas aos demais picos, caso existam.

Podem ser analisadas em uma simulação computacional, tanto a distribuição do solvente ao redor de um átomo específico quanto ao redor do soluto como um todo. Para este último propósito em geral a RDF dos centros de massa entre soluto e solvente são analisadas. Entretanto, no caso de moléculas alongadas este tipo de análise pode não ser adequada [226] e uma nova RDF, chamada de MDDF (Minimum Distance Distribution Function) foi proposta [227]. Ela usa no histograma a menor distância entre soluto e solvente e pode ser normalizada usando uma caixa retangular em vez da distribuição esférica usual e por essa razão se ajusta melhor a moléculas planas ou alongadas.

Neste trabalho, a MDDF foi usada para definir as camadas de solvatação, calculada usando o programa "order", que faz parte do conjunto de ferramentas de análise do programa DICE [228]. As demais funções RDF foram calculadas usando a ferramenta "g_rdf' do programa GROMACS.

\subsubsection{Clustering}

O termo "clustering" refere-se a uma técnica geral de mineração de dados usada para agrupar dados de acordo com algum critério de similaridade. No caso específico de simulações computacional de sistemas moleculares, a técnica de clustering é usada para estudar o espaço conformacional, agrupando estruturas semelhantes em grupos menores ou clusters. Assim o nível de complexidade da informação estrutural é 
reduzido o que torna possível revelar padrões escondidos na complexidade dos dados.

Em qualquer algoritmo de clustering uma métrica é usada para estabelecer um critério de distância entre os elementos do conjunto de estruturas a serem analisadas.

Neste trabalho é usado o algoritmo GROMOS [229] implementado na ferramenta g_cluster do programa GROMACS, que consiste basicamente em três passos:

1) Para cada ponto (estrutura) é calculado o número de outros pontos para os quais o RMSD é menor que um determinado raio de corte (vizinhos).

2) O ponto com maior número de vizinhos juntamente com todos os vizinhos formam um cluster.

3) As estruturas que formam o cluster são retiradas do conjunto total de estruturas e o procedimento é repetido até que todas as estruturas tenham sido classificadas.

\subsubsection{Convolução do espectro UV/Vis}

O processo de absorção ou emissão de luz é caracterizado pela transição entre dois estados. A diferença de energia entre os estados $(\Delta E)$ está relacionada com a frequência $(\nu)$, comprimento de onda $(\lambda)$ ou número de onda $(\tilde{\nu})$ da radiação absorvida de acordo com a relação

$$
\Delta E=h \nu=\frac{h c}{\lambda}=h c \tilde{\nu},
$$

onde $h$ é a constante de Planck e $c$ é a velocidade da luz.

A força de oscilador de uma dada transição é uma grandeza adimensional definida com sendo igual a [230]

$$
f_{i}=\frac{8 \pi^{2} m_{e}}{3 e^{2} h^{2}} \tilde{\nu}_{i}\left|\boldsymbol{\mu}_{i}\right|^{2}
$$

onde $\boldsymbol{\mu}_{i}$ é o momento de dipolo de transição.

Em geral $f$ é um número entre zero e um. Transições proibidas têm força de oscilado próximas de zero, enquanto que bandas intensas mostram valores próximos de um.

A convolução de funções de Lorentz aos resultados dos cálculos de energia de excitação em cada configuração obtidas na simulação computacional podem ser usadas a fim de obter um espectro que pode ser comparado ao resultado experimental. O 
ajuste é dado pela expressão

$$
L(\tilde{\nu})=\frac{1}{N} \sum_{i=1}^{n \cdot N} \frac{f_{i} \cdot \delta \tilde{\nu}^{2}}{\left[(\delta \tilde{\nu})^{2}+\left(\tilde{\nu}_{i}-\tilde{\nu}\right)^{2}\right]}
$$

onde $n$ é o número de excitações usadas na convolução, $N$ é o número de configurações e $\delta \tilde{\nu}$ a largura de linha. Em geral, escolhe-se um valor para a largura que ajuste bem os resultados teóricos aos resultados experimentais.

Neste trabalho as convoluções foram realizadas usando a ferramenta "Lorentz", que faz parte do conjunto de programas de análise do programa DICE. 
$\prod_{\text {Capítulo }}$

\section{Estrutura eletrônica dos complexos}

Neste capítulo são apresentados resultados obtidos a partir das geometrias otimizadas. Foram calculadas uma série de propriedades, como energia de interação, distribuição de carga e energias de excitação.

Inicialmente são apresentados os resultados para o complexo formado pelo $C_{4}(4 C P)^{+}$com brometo e iodeto. Em seguida, para o complexo formado pelo $\mathrm{C}_{3}$ bis $(4 \mathrm{CP})^{2+}$ com iodeto. Ambos os complexos foram estudados em vácuo, ou seja, resolvendo o problema eletrônico sem a inclusão do efeito de solvente ou temperatura. A fim de iniciar o estudo do efeito do solvente, os resultados também foram estendidos para os complexos em solução usando o modelo contínuo polarizável. Todos os cálculos foram realizados com o programa Gaussian 09 [231]. 


\subsection{Complexos $C_{4}(4 C P)^{+} X^{-}$}

Como já foi ressaltado anteriormente no estudo teórico dos complexos de transferência de carga estão envolvidas uma série de questões técnicas (ver seção 1.4 e seção 2.8.7). Vimos que os complexos são sintetizados tendo como contra-íon ânions $\mathrm{Br}^{-}$ e que a banda de transferência de carga aparece em $400 \mathrm{~nm}$, após a adição de $I^{-}$na solução. Por isso, para se certificar que o $\mathrm{Br}^{-}$não tem efeito sobre esta banda, foram estudados inicialmente complexos formados pelo $C_{4}(4 C P)^{+}$tendo como contra-íons tanto o brometo $\left(X^{-}=B r^{-}\right)$quanto o iodeto $\left(X^{-}=I^{-}\right)$.

Vale ressaltar também que no estudo teórico o complexo é considerado como uma única entidade, dentro da chamada abordagem de supermolécula. Assim, apesar da separação de carga existente, o sistema com um todo é neutro (carga total nula). Além disso, em todos os complexos o número total de elétrons é par, o que permite tratá-los no estado singleto (multiplicidade de spin 1) como sistemas camada fechada e considerar ocupação dupla em todos os orbitais (métodos restritos). Por meio de cálculos da energia, verificou-se que o uso de métodos não restritos leva a exatamente o mesmo resultado. Inicialmente o estado tripleto também foi considerado (multiplicidade de spin 3), mas este possuía energia muito mais alta (cerca de $40 \mathrm{kcal} / \mathrm{mol}$ ) em relação ao estado singleto, sendo portanto desconsiderado.

Assim o estado fundamental corresponde a um estado singleto onde, como veremos mais adiante (seção 4.1.2), a carga negativa se localizando sobre o átomo de $I^{-} \mathrm{e}$ carga positiva no anel.

\subsubsection{Geometria de equilíbrio}

Infelizmente existe pouca informação experimental sobre a estrutura dos sistemas. Por isso buscamos diferentes geometrias para os complexos $C_{4}(4 C P)^{+} I^{-}$e $\mathrm{C}_{4}(4 \mathrm{CP})^{+} \mathrm{Br}^{-}$posicionando os contra-íons em diferentes posições, próximas ao anel aromático.

As geometrias de equilíbrio foram obtidas usando o funcional híbrido B3LYP. O conjunto de função base $6-311+G(d, p)$ foi usado para descrever os átomos de $\mathrm{H}, \mathrm{C}$ e N. Trabalhos na literatura mostram que esse nível de cálculo consegue descrever a 

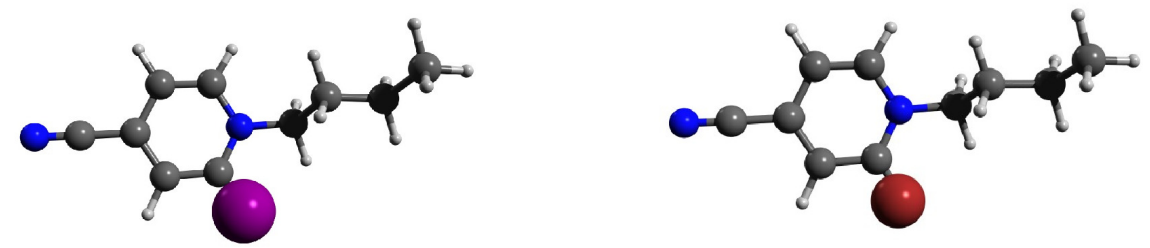

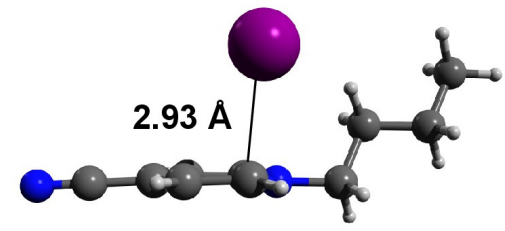

(a)

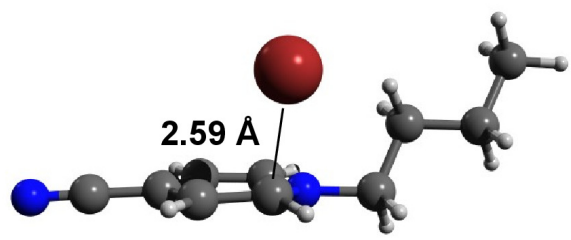

(b)

Figura 4.1: Visão superior (acima) e lateral (abaixo) dos complexos formados pelo $C_{4}(4 C P)^{+} X^{-}$, onde (a) $X^{-}=I^{-}$e (b) $X^{-}=B r^{-}$. As geometrias foram obtidas usando o funcional B3LYP. Bases 6-311+G(d,p) para $\mathrm{C}, \mathrm{N}$,H e aug-cc-PVDZPP para I, Br.

geometria de sistemas molecular com um bom compromisso entre custo computacional e acurácia [232].

Os átomos de bromo e iodo, que possuem respectivamente 35 e 53 elétrons, foram descritos usando o pseudopotencial aug-cc-PVDZ-PP, que foi escolhido por produzir bons resultados para sistemas contendo halogênios [233-235]. Nesse caso apenas 25 elétrons são tratados explicitamente (os 7 elétrons da camada de valência e os 18 da camada interna). A introdução desse pseudopotencial, no entanto, não compromete os resultados, pois verificamos que produz resultados equivalentes aos que são obtidos usando a base $6-311+\mathrm{G}(\mathrm{d}, \mathrm{p})$, a um custo computacional menor.

As configurações de mínima energia encontradas para os complexos em vácuo são apresentadas na Figura 4.1. As coordenadas cartesianas de cada estrutura podem ser encontradas no Apêndice A.1. É interessante notar que tanto o iodeto quanto o brometo estão localizados sobre o anel aromático, mas deslocados do centro do anel, interagindo com o carbono na posição orto do anel aromático. Isso acontece por que o nitrogênio é mais eletronegativo do que o carbono, atraindo para si a nuvem eletrônica e tornando aquela região mais positiva.

Na Tabela 4.1 são apresentadas as energias de interação para cada estrutura, 
Tabela 4.1: Energias de interação $\left(\Delta E_{\text {int }}\right)$ e erro de superposição de base $\left(\mathrm{E}_{C P}\right)$, calculados usando o nível de cálculo B3LYP. Bases 6-311+G(d,p) para C, N, H e aug-cc-PVDZ-PP para I, Br.

\begin{tabular}{ccc}
\hline \hline Propriedade $\left(\right.$ kcal.mol $\left.^{-1}\right)$ & $C_{4}(4 C P)^{+} \mathrm{Br}^{-}$ & $C_{4}(4 C P)^{+} I^{-}$ \\
\hline$\Delta \mathrm{E}_{\text {int }}$ & $-94.8\left(-97.4^{1},-98.4^{2}\right)$ & $-89.6\left(-91.2^{1},-92.6^{2}\right)$ \\
$E_{C P}$ & 0.43 & 0.35 \\
\hline \hline
\end{tabular}

${ }^{1} \mathrm{MP} 2{ }^{2} \mathrm{M} 06-2 \mathrm{X}[238]$

calculadas subtraindo da energia do complexo as energias dos monômeros, ou seja,

$$
\Delta E_{\text {int }}=E_{\text {complexo }}-E_{C_{4}(4 C P)^{+}}-E_{X^{-}},
$$

onde tanto o complexo quanto os monômeros foram considerados em sua geometria otimizada. As correções para o erro de superposição de base, estimadas a partir da correção de Counterpoise $[236,237]\left(\delta E_{C P}\right)$ foram muito pequenas (inferiores a 0.5 kcal.mol ${ }^{-1}$ ) e podem ser desprezadas. Ambos os complexos apresentaram uma energia de interação alta em vácuo (em módulo), e o valor negativo indica grande estabilidade.

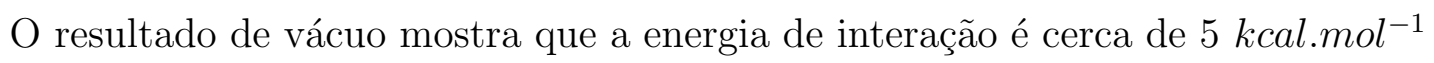
maior no complexo com brometo do que no complexo com iodeto. Em solução, no entanto, a polarização causada pelo ambiente tende a dissociá-los, fazendo com que os complexos sejam fracamente ligados [239]. Isto é corroborado também pelos resultados teóricos, conforme será visto mais adiante (ver seção 4.1.4). É importante ressaltar que determinar a estabilidade dos complexos em solução é um problema muito mais delicado, já que essa estabilidade não é governada apenas pela energia de interação, mas também por termos adicionais envolvendo a interação de cada espécie com o meio solvente, por exemplo. Assim, apenas através da diferença observada em vácuo não é possível estabelecer a estabilidade relativa em solução.

\subsubsection{Densidade de carga e análise de população}

A distribuição eletrônica do $C_{4}(4 C P)^{+} I^{-}$foi estudada por meio de mapas para o potencial eletrostático molecular (MEP, do inglês "molecular electrostatic potential") 
e cálculos da população eletrônica usando o método de cargas naturais [240]. O MEP é construído calculando a força gerada pela distribuição de cargas moleculares em uma carga de prova colocada na vizinhança da molécula e fornece informação visual da distribuição de cargas na molécula, permitindo identificar regiões com ausência ou acúmulo de carga. O mapa do potencial eletrostático apresentado na Figura 4.2, mostra que a superfície de carga negativa (na cor vermelha) se concentram sobretudo sobre o iodeto e o nitrogênio do grupo nitrila. Como o objetivo é apenas ilustrar a distribuição de carga no complexo, o cálculo foi realizado apenas no nível HF/3-21G.

Já as regiões de carga positiva (representadas na cor azul na figura) se localizam sobretudo sobre os hidrogênios do anel. Todo o anel aromático possui uma carga positiva, sobretudo nas posições do anel vizinhas ao nitrogênio aromático (posições orto do anel), onde ocorre a interação com o iodeto. A cadeia carbônica é uma região bastante apolar (carga neutra).

Na Figura 4.2 é apresentado um mapa de diferença de densidade eletrônica entre o primeiro estado excitado e o estado fundamental. Esse mapa é interessante pois permite avaliar transferências de carga ocorridas na excitação eletrônica entre esses níveis de energia. Em azul estão as regiões que perdem carga na excitação, enquanto que em roxo são as regiões que ganham carga. Fica claro que, em uma excitação eletrônica do estado fundamental para o primeiro estado excitado, a carga é transferida do iodeto para o anel aromático, como é o comportamento típico esperado para esse sistema. Estas excitações eletrônicas serão discutidas em detalhes na próxima seção.

O MEP corresponde a uma visão global da distribuição de cargas na molécula, mas permite apenas uma análise qualitativa. Uma análise quantitativa foi realizada a partir dos métodos de análise de população eletrônica.

Usando os métodos de análise de população é possível associar uma carga (em unidades de carga elétrica) a cada núcleo baseando-se na distribuição eletrônica. Como a carga atômica não é um observável na mecânica quântica, não é possível definir um operador para a carga elétrica. A partir da função de onda eletrônica é possível apenas determinar uma densidade eletrônica contínua a partir da qual não existe uma forma única para particionar as cargas eletrônicas (cargas parciais) entre os fragmentos do sistema, sejam átomos ou moléculas. Por isso existem diversos métodos de análise de população disponíveis e alguns dos mais usados no estudo de sistemas moleculares são 


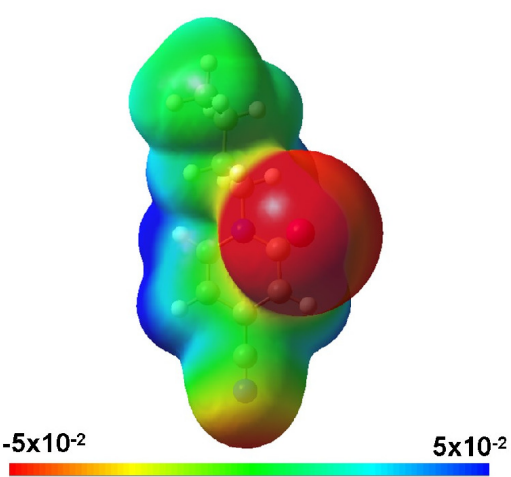

(a)

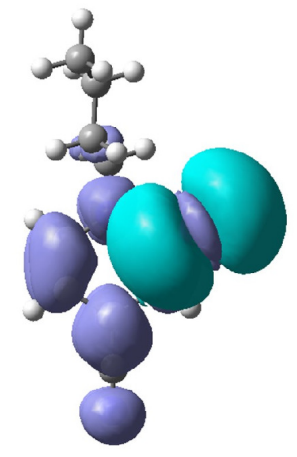

(b)

Figura 4.2: (a) Mapa da densidade eletrônica no complexo $C_{4}(4 C P)^{+} I^{-}$. (b) Mapa da diferença de densidade eletrônica entre o primeiro estado excitado e o estado fundamental do mesmo complexo. Cálculos realizados no nível HF/3-21G. Unidades atômicas $\left(e / b o h r^{3}\right)$.

discutidos na referência [241].

No caso dos CTC, as cargas parciais são melhores descritas usando o método de população natural NPA (do inglês "Natural Population Analysis") [240]. No método NPA as cargas atômicas são obtidas a partir dos chamados orbitais naturais, autovetores da matriz densidade reduzida de uma partícula. Já os autovalores da matriz são os números de ocupação (entre 0 e 2) e são usados como base para definir as chamadas cargas atômicas naturais em cada átomo.

As cargas naturais calculadas usando o método NPA são apresentadas na Figura 4.3, superpostas aos respectivos átomos. Para facilitar a visualização as cargas de cada grupo $\mathrm{CH} 2$ ou $\mathrm{CH} 3$ da cadeia foram somadas, resultando em uma carga quase nula sobre cada carbono da cadeia alifática.

É interessante notar que em vácuo ocorre uma transferência considerável da carga do contra-íon para o anel aromático já no estado fundamental. As cargas no iodeto e brometo são -0.675 e -0.633. No cálculo em acetonitrila, usando o modelo contínuo PCM, o valor é de -0.845 e -0.784, respectivamente, de modo que a transferência de carga é menos expressiva.

O nitrogênio do anel possui carga negativa: -0.259 para o $C_{4}(4 C P)^{+}$e -0.322 nos 


\section{Vácuo}
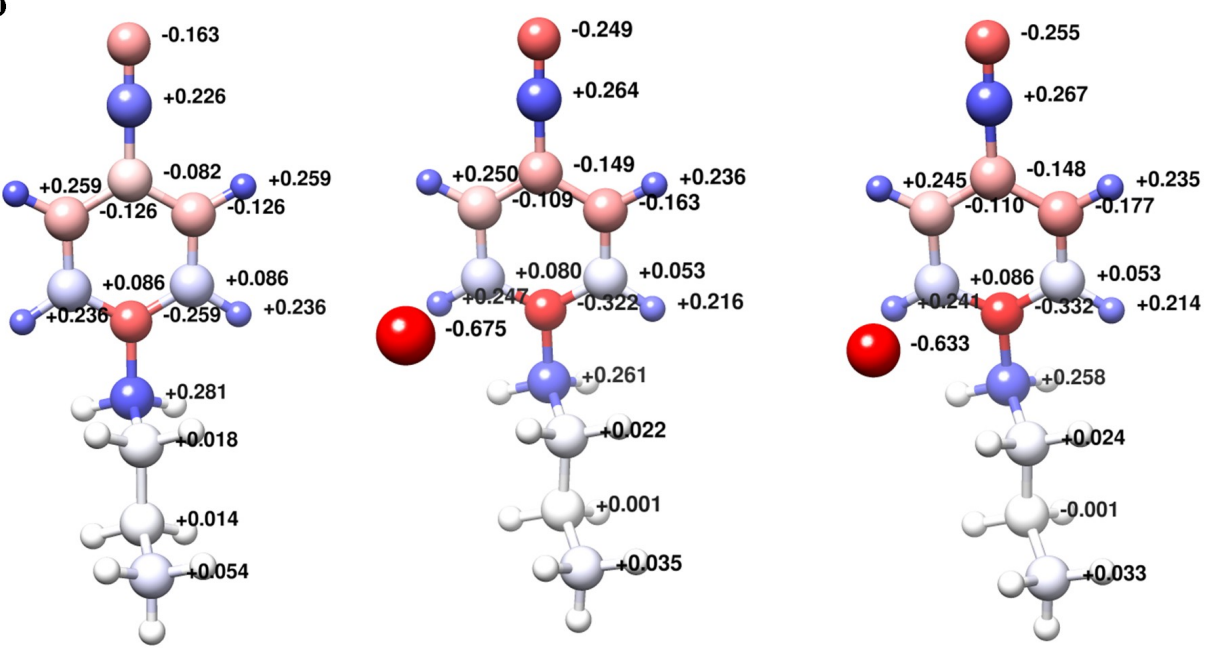

\section{Acetonitrila}

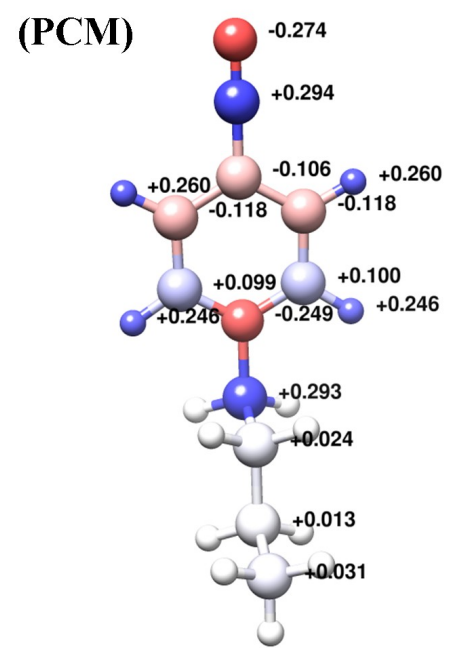

$\mathrm{C}_{4}(\mathbf{4 C P})^{+}$

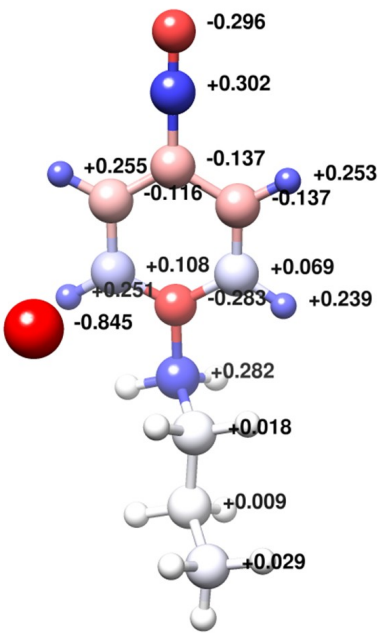

$\mathrm{C}_{4}(4 \mathrm{CP})^{+} \mathrm{I}^{-}$

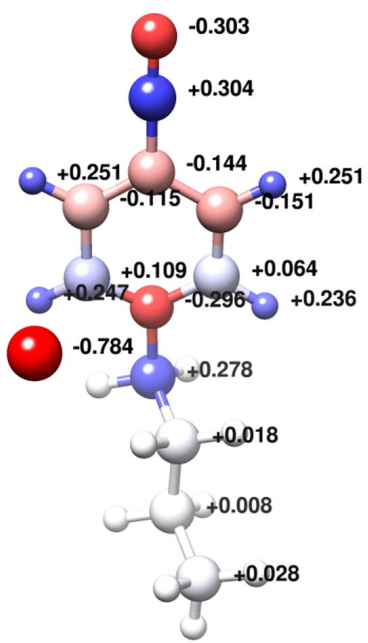

$\mathrm{C}_{4}(4 \mathrm{CP})^{+} \mathrm{Br}^{-}$

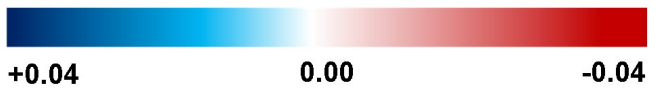

Figura 4.3: Cargas naturais para o $C_{4}(4 C P)^{-}$e seus complexos com iodeto e brometo, em vácuo e em acetonitrila $(\mathrm{PCM})$. Cálculos no nível MP2. Bases $6-311+\mathrm{G}(\mathrm{d}, \mathrm{p})$ para $\mathrm{C}, \mathrm{H}$, $\mathrm{N}$ e aug-cc-PVDZ-PP para Br ou I. Os átomos da cadeia carbônica são representados pela carga do grupo (CH2 ou $\mathrm{CH} 3)$, ou seja, a carga dos hidrogênios foram somadas às cargas do carbono. 
complexos, ambos em vácuo. Como resultado, os carbonos na posição orto, ligados a este nitrogênio, ficam com carga positiva, o que explica a interação do contra-íon nesta posição.

A carga em cada um dos grupos $\mathrm{CH} 2$ ou $\mathrm{CH} 3$ da cadeia é praticamente nula, o que explica porque o iodeto prefere interagir com o anel e é consistente com o fato do tamanho da cadeia não alterar significativamente o espectro eletrônico.

A formação do complexo afeta a distribuição de cargas do anel, devido o efeito de polarização e da carga transferida do contra-íon ao $C_{4}(4 C P)^{+}$. A maior variação de carga acontece no nitrogênio do grupo nitrila, cuja carga varia de -0.09 (-0.02 em acetonitrila). O nitrogênio aromático sofre variação de -0.06 em vácuo (-0.03 em solução).

Já as variações de cargas nos hidrogênios são pequenas, de no máximo 0.02 em vácuo (0.01 em solução). Os carbonos do anel se tornam mais negativos com a presença do iodeto (sofrendo variações no intervalo de -0.01 a -0.07 em vácuo e de -0.01 a -0.03 em solução).

Avaliar a distribuição de carga é importante também para as simulações computacionais usando a dinâmica molecular clássica. Os principais campos de força disponíveis na literatura usam um conjunto de cargas fixas para tratar o sistema. Nossos resultados nessa seção mostram que ocorrem processos de polarização e transferência parcial de carga, de forma que os campos de força podem ter dificuldades em descrever a estabilidade dos complexos, sobretudo em vácuo. Felizmente esses efeitos são menos pronunciados em acetonitrila.

\subsubsection{Energias de excitação}

Como foi discutido anteriormente (ver seções 1.4 e 2.8.7), excitações de transferência de carga podem ser um grande desafio para os métodos teóricos. Por isso, inicialmente as três primeiras excitações eletrônicas foram calculadas usando diversos métodos teóricos distintos, tanto com a TDDFT e diversos funcionais (Tabela 4.2) quanto com alguns métodos baseados em função de onda: TDHF, CIS, CIS(D) e EOM-CCSD.

Em relação aos cálculos usando a TDDFT, foram usados os funcionais tradi- 
cionais da família BLYP e PBE, puros e híbridos (B3LYP, BHandHLYP), além de funcionais com correção de longo alcance, especialmente desenvolvidos para lidar com excitações de transferência de carga: puros (LC-BLYP e LC- $\omega$ PBE) e híbridos (CAMB3LYP, $\omega$ B97X-D). Os resultados para as três energias de excitação de mais baixa energia (em comprimento de onda) são apresentados na Tabela 4.2, juntamente com suas respectivas força de oscilador $(f)$.

Em princípio, o valor mais confiável apresentado na tabela é o calculado com EOM-CCSD [242-245]. Esse método é considerado um dos mais acurados para o estudo das energias de excitação de moléculas. Entretanto, seu custo computacional é altíssimo, crescendo com a sexta potência no número de elétrons do sistema $\left(N^{6}\right)$, o que inviabiliza sua aplicação nos complexos estudados neste trabalho. Usando uma CPU com oito núcleos de processamento e 46 Gb de memória, o cálculo de apenas duas excitações levou 13 dias. O cálculo foi realizado usando ainda uma base reduzida, a base 6-31G $(\mathrm{d})(\mathrm{C}, \mathrm{H}, \mathrm{N}) / \mathrm{LANL} 2 \mathrm{DZ}(\mathrm{I})$, pois o cálculo com a base $6-311 \mathrm{G}+(\mathrm{d}, \mathrm{p})$ não convergia. Em comparação, o cálculo usando TDDFT na mesma base não leva mais do que 30 minutos.

Vamos nos concentrar inicialmente nos resultados para o complexo $C_{4}(4 C P)^{+} I^{-}$. As três primeiras excitações calculadas com EOM-CCSD são 550, 547 e $510 \mathrm{~nm}$. As duas primeiras são quase degeneradas e têm intensidade quase nula $(f=0.01)$, enquanto que terceira excitação é a de maior intensidade $(f=0.26)$. Não encontramos na literatura resultados experimentais para a banda de transferência de carga do complexo $C_{4}(4 C P)^{+} I^{-}$na fase gasosa, mas existem valores para solventes de baixa polaridade: $495 \mathrm{~nm}$ (clorofórmio) e $529 \mathrm{~nm}$ (benzeno) [246]. Observa-se, portanto, uma concordância entre a terceira excitação (a mais intensa) e o valor medido nestes solventes. $\mathrm{O}$ caráter dessas excitações será discutido um pouco mais adiante.

Os demais métodos apresentaram qualitativamente resultados semelhantes, mas quantitativamente o valor das excitações varia consideravelmente. É interessante olhar também para os valores obtidos com HF e CIS. Sabe-se que devido ao pobre tratamento da correlação eletrônica nesses métodos, a tendência é superestimar a energia de excitação (subestimando, portanto, o comprimento de onda) [247]. Apesar disso, eles conseguem lidar com excitações de transferência de carga, servindo de referência como um limite superior, por exemplo. O teorema de Brillouin mostra que determinantes 
Tabela 4.2: Comprimento de onda $(\lambda \mathrm{em} \mathrm{nm})$ e respectivas força de oscilador $(f)$ para as três excitações eletrônicas de mais baixa energia, calculadas em vácuo, do complexo $C_{4}(4 C P)^{+} X^{-}\left(X^{-}=B r^{-}, I^{-}\right)$usando diferentes métodos teóricos. Bases 6-311+G(d,p) para $\mathrm{C}, \mathrm{N}, \mathrm{H}$ e aug-cc-PVDZ-PP para I, Br.

\begin{tabular}{|c|c|c|c|c|c|c|}
\hline & \multicolumn{2}{|c|}{ Transição 1} & \multicolumn{2}{|c|}{ Transição 2} & \multicolumn{2}{|c|}{ Transição 3} \\
\hline & $\lambda$ & $f$ & $\lambda$ & $f$ & $\lambda$ & $f$ \\
\hline \multicolumn{7}{|l|}{ Iodeto } \\
\hline TDHF & 368 & 0.31 & 339 & 0.01 & 335 & 0.01 \\
\hline CIS & 352 & 0.36 & 336 & 0.02 & 332 & 0.02 \\
\hline CIS(D) & 634 & 0.36 & 699 & 0.02 & 695 & 0.02 \\
\hline PBE & 891 & 0.00 & 842 & 0.00 & 589 & 0.05 \\
\hline B3LYP & 733 & 0.00 & 713 & 0.00 & 537 & 0.10 \\
\hline BhandHLYP & 544 & 0.00 & 536 & 0.00 & 466 & 0.20 \\
\hline LC-BLYP & 443 & 0.06 & 436 & 0.02 & 424 & 0.19 \\
\hline LC- $\omega$ PBE & 457 & 0.03 & 450 & 0.01 & 432 & 0.22 \\
\hline CAM-B3LYP & 568 & 0.00 & 559 & 0.00 & 480 & 0.18 \\
\hline$\omega B 97 X-D$ & 557 & 0.00 & 548 & 0.00 & 477 & 0.19 \\
\hline $\mathrm{EOM}^{-C C S D}{ }^{1}$ & 550 & 0.01 & 547 & 0.01 & 510 & 0.26 \\
\hline \multicolumn{7}{|l|}{ Brometo } \\
\hline TDHF & 318 & 0.29 & 275 & 0.01 & 272 & 0.00 \\
\hline CIS & 302 & 0.38 & 272 & 0.01 & 269 & 0.00 \\
\hline CIS(D) & 442 & 0.38 & 481 & 0.01 & 477 & 0.00 \\
\hline PBE & 643 & 0.00 & 613 & 0.00 & 495 & 0.05 \\
\hline B3LYP & 526 & 0.00 & 515 & 0.00 & 448 & 0.08 \\
\hline BhandHLYP & 403 & 0.01 & 400 & 0.02 & 384 & 0.14 \\
\hline LC-BLYP & 363 & 0.21 & 348 & 0.01 & 344 & 0.02 \\
\hline LC- $\omega$ PBE & 370 & 0.17 & 360 & 0.01 & 355 & 0.03 \\
\hline CAM-B3LYP & 425 & 0.01 & 420 & 0.01 & 399 & 0.13 \\
\hline$\omega B 97 X-D$ & 419 & 0.01 & 415 & 0.01 & 396 & 0.13 \\
\hline $\mathrm{EOM}-\mathrm{CCSD}^{1}$ & 414 & 0.15 & 408 & 0.00 & 399 & 0.08 \\
\hline
\end{tabular}




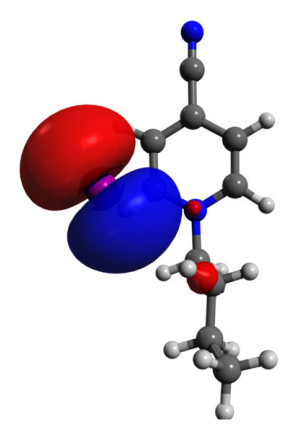

HOMO-2

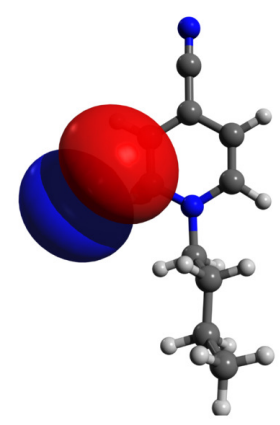

HOMO-1

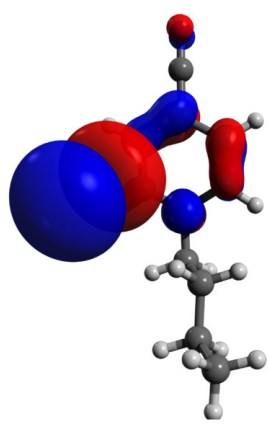

HOMO

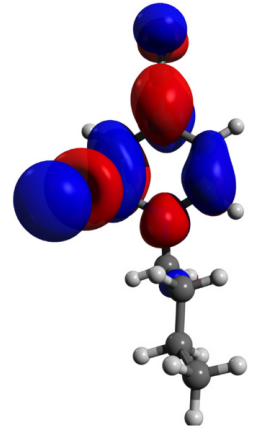

LUMO

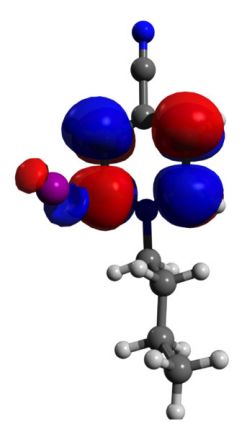

$\mathrm{LUMO}+1$

Figura 4.4: Principais orbitais moleculares envolvidos nas transições de transferência de carga do $C_{4}(4 C P)^{+} I^{-}$. Calculados usando CAM-B3LYP/3-21G.

mono excitados são ortogonais ao estado fundamental [158]. Assim CIS e TDHF, embora usem abordagens distintas, produzem essencialmente os mesmo resultados. Note que há uma inversão na ordem das excitações: a mais intensa é a de mais baixa energia, localizada em $368 \mathrm{~nm}$ (TDHF) ou $352 \mathrm{~nm}$ (CIS). A inclusão perturbativa das excitações duplas, feitas no método CIS(D), desloca excessivamente o resultado para menores comprimentos de onda $(634 \mathrm{~nm})$ : em relação ao valor de $510 \mathrm{~nm}$ calculado EOM-CCSD há uma diferença de mais de $100 \mathrm{~nm}$.

Em relação a TDDFT, conforme já era esperado, PBE e B3LYP subestimam consideravelmente as energias de excitação (superestimam o comprimento de onda). Estes funcionais chegam a apresentar excitações com comprimento onda superior a $700 \mathrm{~nm}$ (ainda que sejam as excitações com intensidade nula), embora a excitação mais intensa, $589 \mathrm{~nm}$ (PBE) ou $537 \mathrm{~nm}$ (B3LYP), seja próxima do valor de referência (510 nm). Observa-se também que aumentar a contribuição do termo de troca de HF de 30\% (B3LYP) para 50\% (BHandHLYP) melhora consideravelmente a descrição da excitação, e os resultados já aparecem na região de 450 - $550 \mathrm{~nm}$.

Mas a melhor concordância com os resultados obtidos com EOM-CCSD foi obtida usando os funcionais com correções de longo alcance, principalmente os funcionais CAM-B3LYP e $\omega$ B97X-D. Os outros dois funcionais com correção de longo alcance, LC-BLYP e LC- $\omega$ PBE, apresentam resultados superestimados em 80-90 nm. A razão é que o termo de HF é melhor controlado nos funcionais CAM-B3LYP e $\omega$ B97X-D do que nos funcionais LC-BLYP e LC- $\omega$ PBE. 
Tabela 4.3: Energias de excitação $E_{\text {exc }}$ e respectivos comprimento de onda para o complexo $C_{4}(4 C P)^{+} I^{-}$usando o método EOM-CCSD e os funcionais CAM-B3LYP e $\omega$ B97X-D. Força de oscilador entre parênteses.

\begin{tabular}{|c|c|c|c|}
\hline Transição & $E_{\text {exc }}(\mathrm{eV})$ & $\lambda(\mathrm{nm})$ & Composição \\
\hline \multicolumn{4}{|c|}{ EOM-CCSD $^{1}$} \\
\hline 1 & 2.24 & $553(0.01)$ & $\mathrm{H}-1 \rightarrow \mathrm{LUMO}(79 \%), \mathrm{HOMO} \rightarrow \mathrm{LUMO}(7 \%)$ \\
\hline 2 & 2.26 & $549(0.01)$ & $\mathrm{H}-2 \rightarrow \mathrm{LUMO}(83 \%), \mathrm{H} \rightarrow \mathrm{LUMO}(2 \%)$ \\
\hline 3 & 2.42 & $511(0.26)$ & $\mathrm{HOMO} \rightarrow \mathrm{LUMO}(74 \%), \mathrm{H}-1 \rightarrow$ LUMO $(6 \%)$ \\
\hline 4 & 3.20 & $387(0.05)$ & $\mathrm{HOMO} \rightarrow \mathrm{L}+2(31 \%), \mathrm{HOMO} \rightarrow \mathrm{L}+3(41 \%)$ \\
\hline 5 & 3.62 & $342(0.00)$ & $\mathrm{H}-1 \rightarrow \mathrm{L}+2(22 \%), \mathrm{H}-1 \rightarrow \mathrm{L}+3(27 \%)$ \\
\hline 6 & 3.71 & $334(0.12)$ & $\mathrm{H}-2 \rightarrow \mathrm{L}+2(21 \%), \mathrm{H}-2 \rightarrow \mathrm{L}+3(27 \%)$ \\
\hline \multicolumn{4}{|c|}{ CAM-B3LYP } \\
\hline 1 & 2.18 & $568(0.00)$ & $\mathrm{H}-1 \rightarrow \mathrm{LUMO}(88 \%), \mathrm{HOMO} \rightarrow \mathrm{LUMO}(7 \%)$ \\
\hline 2 & 2.22 & $559(0.00)$ & $\mathrm{H}-2 \rightarrow \mathrm{LUMO}(95 \%), \mathrm{H}-1 \rightarrow \mathrm{LUMO}(2 \%)$ \\
\hline 3 & 2.58 & $480(0.18)$ & $\mathrm{HOMO} \rightarrow \mathrm{LUMO}(87 \%), \mathrm{H}-1 \rightarrow \mathrm{LUMO}(7 \%)$ \\
\hline 4 & 3.28 & $377(0.06)$ & $\mathrm{HOMO} \rightarrow \mathrm{L}+1(93 \%), \mathrm{H}-2 \rightarrow \mathrm{L}+1(2 \%)$ \\
\hline 5 & 3.63 & $342(0.00)$ & $\mathrm{H}-2 \rightarrow \mathrm{L}+1(13 \%), \mathrm{H}-1 \rightarrow \mathrm{L}+1(84 \%)$ \\
\hline 6 & 3.81 & $325(0.15)$ & $\mathrm{H}-2 \rightarrow \mathrm{L}+1(78 \%), \mathrm{H}-1 \rightarrow \mathrm{L}+1(12 \%)$ \\
\hline \multicolumn{4}{|l|}{$\omega \mathrm{B} 97 \mathrm{X}-\mathrm{D}$} \\
\hline 1 & 2.22 & $557(0.00)$ & $\mathrm{H}-1 \rightarrow \mathrm{LUMO}(88 \%), \mathrm{H}-2 \rightarrow \mathrm{LUMO}(2 \%)$ \\
\hline 2 & 2.26 & $548(0.00)$ & $\mathrm{H}-2 \rightarrow \mathrm{LUMO}(95 \%), \mathrm{H}-1 \rightarrow \mathrm{LUMO}(2 \%)$ \\
\hline 3 & 2.60 & $477(0.19)$ & $\mathrm{HOMO} \rightarrow \mathrm{LUMO}(87 \%), \mathrm{H}-1 \rightarrow \mathrm{LUMO}(7 \%)$ \\
\hline 4 & 3.31 & $375(0.06)$ & $\mathrm{HOMO} \rightarrow \mathrm{L}+1(94 \%), \mathrm{H}-2 \rightarrow \mathrm{L}+1(2 \%)$ \\
\hline 5 & 3.67 & $337(0.00)$ & $\mathrm{H}-2 \rightarrow \mathrm{L}+1(14 \%), \mathrm{H}-1 \rightarrow \mathrm{L}+1(82 \%)$ \\
\hline 6 & 3.85 & $322(0.15)$ & $\mathrm{H}-2 \rightarrow \mathrm{L}+1(77 \%), \mathrm{H}-1 \rightarrow \mathrm{L}+1(13 \%)$ \\
\hline
\end{tabular}


As excitações calculadas para o $C_{4}(4 C P)^{+} B r^{-}$, também apresentadas na Tabela 4.2, seguem o comportamento das excitações do complexo com $I^{-}$, uma vez que ambos pertencem ao grupo dos halogênios, possuindo 7 elétrons de valência, e apresentando portanto propriedades físico-químicas semelhantes. As excitações do complexo com $\mathrm{Br}^{-}$são cerca de $100 \mathrm{~nm}$ menores em relação ao complexos com $I^{-}$, de forma que não se espera que a banda do primeiro afete a do segundo.

A Tabela 4.3 apresenta uma janela de 6 excitações para o $C_{4}(4 C P)^{+} I^{-}$, calculadas usando os funcionais CAM-B3LYP e $\omega$ B97X-D e o método EOM-CCSD. Com base na composição é possível classificar as transições eletrônicas: a primeira como do tipo $\mathrm{H}-1 \rightarrow \mathrm{LUMO}$, a segunda como $\mathrm{H}-2 \rightarrow \mathrm{LUMO}$, a terceira como HOMO $\rightarrow$ LUMO, a quarta como $\mathrm{HOMO} \rightarrow \mathrm{L}+1$, a quinta como $\mathrm{H}-1 \rightarrow \mathrm{L}+1$ e finalmente, a sexta $\mathrm{H}-2 \rightarrow \mathrm{L}+1$.

Aqui foi empregada a nomenclatura usual onde os orbitais ocupados são classificados em ordem decrescente de energia como HOMO (do inglês "highest occupied molecular orbital"), HOMO-1 (H-1), HOMO-1 (H-2), etc.; e os orbitais desocupados são classificados em ordem crescente de energia como LUMO (do inglês "lowest unoccupied molecular orbital"), LUMO+1 (L+1), LUMO+2 (L+2), etc.

A reprentação visual dos orbitais moleculares envolvidos nestas transições, calculados com CAM-B3LYP, é apresentada na Figura 4.4. Os orbitais ocupados (HOMO, HOMO-1 e HOMO-2) são localizados predominantemente sobre o átomo de iodo e se assemelham aos orbitais atômicos $p_{x}, p_{y}$ e $p_{z}$. Já os orbitais desocupados (LUMO e LUMO+1) são localizados sobre o anel aromático do $C_{4}(4 C P)^{+}$. Nas 6 transições, portanto, ocorre a transferência da carga localizada sobre o iodo para o anel aromático.

Os resultados da Tabela 4.3 também mostram que o espectro do $C_{4}(4 C P)^{+} I^{-}$ é composto por duas bandas de transferência de carga. A primeira banda aparece na região de 560 - $480 \mathrm{~nm}$ e é formada pelas três primeiras excitações, caracterizadas pela transferência da carga localizada majoritariamente sobre os orbitais do átomo de iodo para o orbital LUMO localizado no anel. A segunda banda aparece na região de 380-320 nm é formada pelas próximas três transições caracterizadas pela transferência da carga localizada sobre os orbitais do átomo de iodo para o orbital $\mathrm{L}+1$ localizado no anel.

A existência de duas bandas de transferência de carga para o complexo é corroborada por resultados experimentais [40,248,249], que mostram o aparecimento da 
segunda banda em solventes de baixa polaridade. Com o aumento da polaridade as duas bandas se deslocam para o azul (maiores energias), e a banda mais energética é superposta pela banda de absorção do piridínio. Por essa razão em geral o interesse experimental reside na banda de maior comprimento de onda. Outro fato interessante é que apesar da banda de maior comprimento de onda ser composta por três transições, somente a transição $\mathrm{HOMO} \rightarrow$ LUMO possui intensidade apreciável.

\subsubsection{Excitações em solução usando o modelo contínuo}

Duas abordagens distintas podem ser usadas para a obtenção dos resultados em solução usando o modelo contínuo PCM. A primeira consiste em calcular a propriedade mantendo a geometria de vácuo. A segunda consiste em primeiro buscar a configuração de equilíbrio em solução para depois fazer o cálculo da propriedade.

Usando essas duas abordagens, as três primeiras excitações para os complexos foram calculadas em acetonitrila usando os funcionais B3LYP, LC-BLYP, CAM-B3LYP e $\omega$ B97X-D. Os resultados são apresentados na Tabela 4.4.

Os melhores resultados foram obtidos mantendo a geometria de vácuo e realizando o cálculo das transições eletrônicas com os funcionais CAM-B3LYP e $\omega$ B97X-D. Estes funcionais apresentaram transições $\mathrm{HOMO} \longrightarrow$ LUMO intensas em cerca de 360 $\mathrm{nm}$ (brometo) e em 405-412 nm (iodeto). O resultado experimental para o complexo $C_{2}(4 C P)^{+} I^{-}$em acetonitrila é de $420 \mathrm{~nm}[246]$.

Embora o resultado teórico mostre o aparecimento de uma excitação em cerca de $360 \mathrm{~nm}$ para o $C_{4}(4 C P)^{+} \mathrm{Br}^{-}$em acetonitrila, essa banda não é observada experimentalmente pois acaba sendo encoberta pela banda muito mais intensa proveniente das excitações do monômero $C_{4}(4 C P)^{+}$, que também são próximas dessa região.

Nas geometrias em solução obtidas com o funcional B3LYP (ver Figura 4.5), os contra-íons $\mathrm{I}^{-}$e $\mathrm{Br}^{-}$se deslocam para o plano do anel e todas as excitações de transferência de carga apresentam intensidade nula. A única transição com intensidade apreciável, de comprimento $246 \mathrm{~nm}(f=0.16)$ para o complexo com $\mathrm{Br}^{-}$, foi obtida com o funcional LC-BLYP e corresponde a uma transição intramolecular no anel aromático.

A estabilidade em solução depende do equilíbrio entre a interação associativa 
Tabela 4.4: Três excitações eletrônicas de mais baixa energia, do complexo $C_{4}(4 C P)^{+} X^{-}$ $\left(X^{-}=I^{-}, B r^{-}\right)$calculadas com a inclusão do efeito do solvente acetonitrila com o modelo contínuo PCM sobre as geometrias obtidas em vácuo e em solução, usando diferentes funcionais.

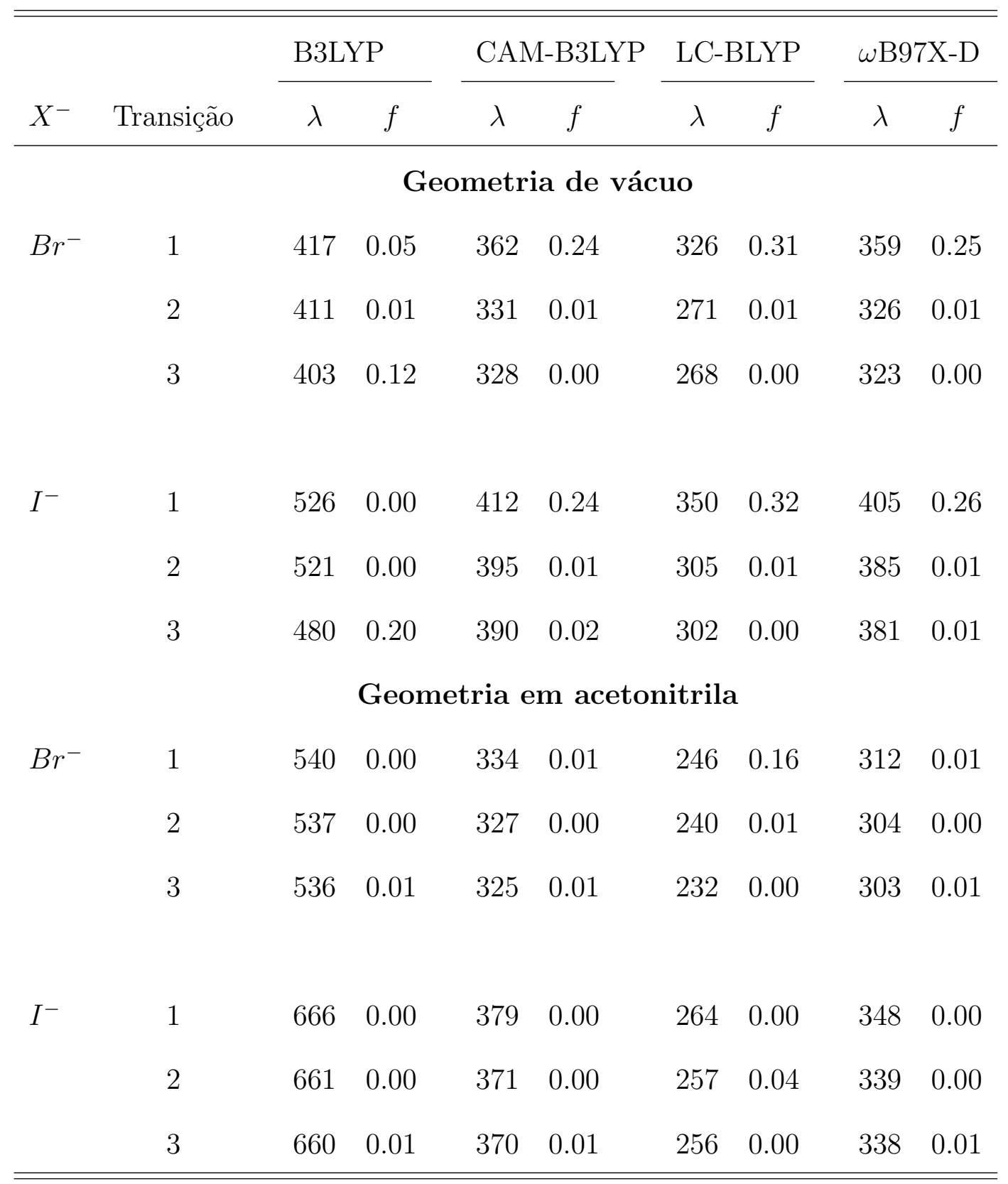



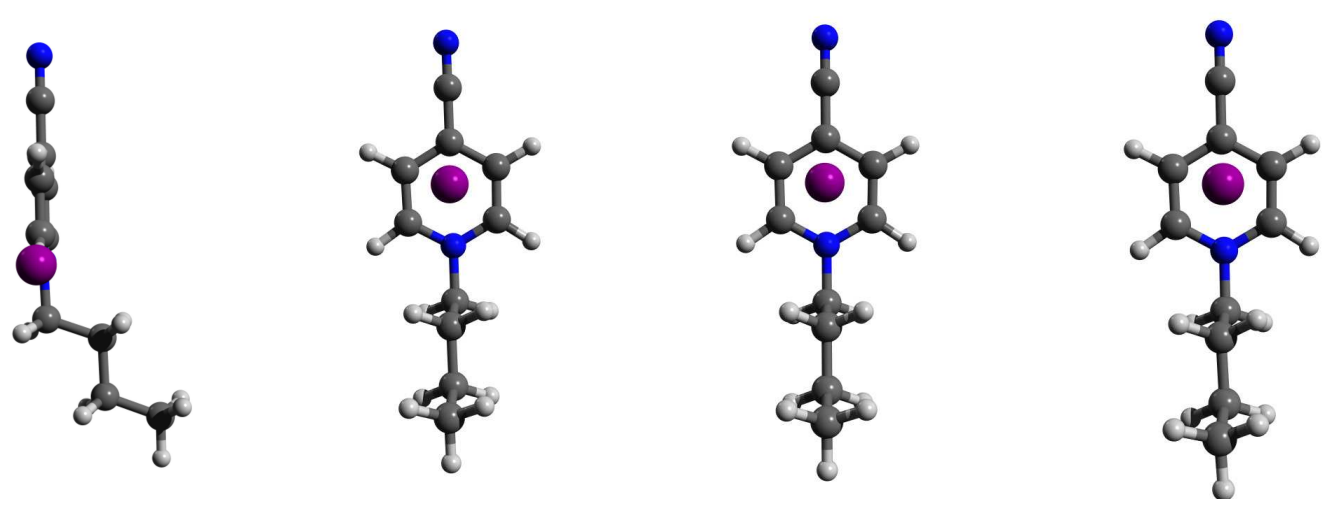

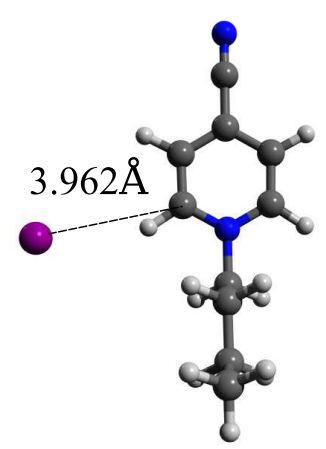

B3LYP

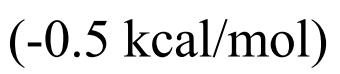

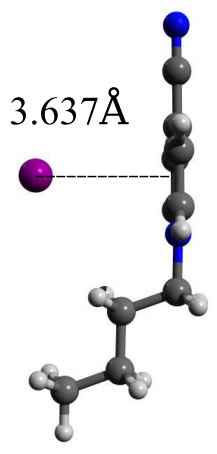

MP2

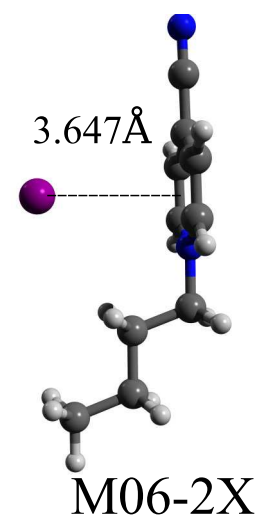

$(-5.8 \mathrm{kcal} / \mathrm{mol})$

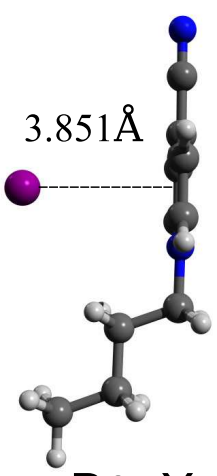

$\omega B 97 X-D$

Figura 4.5: Representação das estruturas de mínima energia encontradas teoricamente para o complexo $C_{4}(4 C P)^{+} I^{-}$em solução (com modelo contínuo PCM), calculadas com diferentes funcionais. A energia de interação, mostrada entre parênteses, foi calculada usando o nível de calculo correspondente.Bases 6-311+G(d,p) para C, N, H e aug-cc-PVDZ-PP para I, Br.

do íons e a interação íon-solvente, que tende a dissociar o complexo. Por isso uma descrição acurada da interação de sistemas iônicos em solução exige um tratamento adequado do termo de dispersão [250]. O fato dos íons irem para o plano do anel na otimização de geometria está associado ao pobre tratamento do termo de dispersão existente no funcional B3LYP.

Na Figura 4.5 são apresentadas a geometrias de solução (acetonitrila, PCM) para o complexo $C_{4}(4 C P)^{+} I^{-}$, obtidas com o funcional B3LYP, MP2 e com funcionais com correções empíricas para o termo de dispersão: M06-2X e $\omega$ B97X-D. Melhorando o tratamento das forças intermoleculares, o $I^{-}$permanece acima do plano do anel, mas a distância ao anel é cerca de 0.7 A maior em relação a geometria de vácuo. Como con- 
Tabela 4.5: Comprimento de onda da excitação eletrônica de mais baixa energia (em nm) e respectiva força de oscilador, calculada para o complexo $C_{4}(4 C P)^{+} I^{-}$em diferentes solventes, no nível PCM-TD-DFT. Bases 6-311+G(d,p) para C, N ,H e aug-cc-PVDZ-PP para I, Br.

\begin{tabular}{|c|c|c|c|c|c|c|}
\hline & \multirow{2}{*}{$\begin{array}{l}\text { Constante } \\
\text { Dielétrica }^{1}\end{array}$} & \multicolumn{2}{|c|}{ CAM-B3LYP } & \multicolumn{2}{|c|}{$\omega \mathrm{B} 97 \mathrm{XD}$} & \multirow{2}{*}{$\begin{array}{c}\operatorname{Exp}^{2} \\
\lambda\end{array}$} \\
\hline & & $\lambda$ & $f$ & $\lambda$ & $f$ & \\
\hline 1,4-Dioxano & 2.2099 & 488 & 0.02 & 479 & 0.05 & 468 \\
\hline Benzeno & 2.2706 & 487 & 0.03 & 477 & 0.07 & 529 \\
\hline Clorofórmio & 4.7113 & 446 & 0.17 & 439 & 0.22 & 495 \\
\hline THF & 7.4257 & 432 & 0.26 & 426 & 0.25 & 520 \\
\hline Acetona & 20.493 & 416 & 0.24 & 409 & 0.26 & 464 \\
\hline Etanol & 24.852 & 414 & 0.24 & 408 & 0.26 & 376 \\
\hline Metanol & 32.613 & 412 & 0.24 & 405 & 0.26 & 360 \\
\hline Acetonitrila & 35.688 & 412 & 0.24 & 405 & 0.26 & 421 \\
\hline DMSO & 46.826 & 412 & 0.25 & 405 & 0.27 & 427 \\
\hline Água & 78.3553 & 408 & 0.25 & 402 & 0.26 & \\
\hline
\end{tabular}

${ }^{1}$ Constante Dielétrica usada no modelo contínuo PCM para o respectivo solvente ${ }^{2}$ valores experimentais para o complexo formado com o $C_{2}(4 C P)^{+}[246]$

sequência, em nenhuma das geometrias foi possível observar transições de transferência de carga com intensidade. A excitação de transferência de carga é observada somente em uma posição bem específica do anel e esta posição fica melhor caracterizada pelas geometrias de vácuo.

Considerando a geometria de vácuo do complexo $C_{4}(4 C P)^{+} I^{-}$e os funcionais CAM-B3LYP e $\omega$ B97X-D, as energias de excitação foram calculadas em outros solventes, usando ainda o modelo contínuo PCM. Os cálculos foram comparados com os resultados experimentais obtidos da literatura para o complexo $C_{2}(4 C P)^{+} I^{-}$e são apresentados na Tabela 4.5. 
Os resultados da Tabela 4.5 mostram claramente que a partir da acetona, o modelo contínuo PCM não muda significativamente o valor para a energia de excitação, que está na região de 412-416 nm. O valor calculado em acetonitrila (412 nm) é muito próximo do valor obtido experimentalmente, mas o mesmo não ocorre em outros solventes como metanol, etanol, acetona, etc.

Portanto, se por um lado o modelo contínuo PCM melhora o valor das energias de excitação em acetonitrila, por outro lado o valor é o mesmo para um número de solventes com polaridade bastante distinta.

Em resumo, os resultados apresentados mostram que os funcionais CAM-B3LYP e $\omega$ B97X-D apresentaram melhor desempenho na descrição da banda de transferência de carga dos complexos.

Nossos resultados também indicam que a inclusão do efeito do solvente é importante para descrever o espectro em solventes de alta polaridade como acetonitrila. $\mathrm{O}$ modelo contínuo PCM apresentou duas limitações importantes. A primeira foi que as excitações calculadas apresentaram o mesmo valor para solventes que vão desde acetona até a água. A outra dificuldade foi a descrição das geometrias em solução, que apresentam intensidade nula para as excitações de transferência de carga. Apesar das limitações, os valores calculados em acetonitrila usando a geometria de vácuo exibiram com acordo com o valor experimental. 


\subsection{Complexo $C_{3} b i s(4 C P)^{2+} I^{-}$}

Nesta seção são apresentados os resultados dos cálculos de estrutura eletrônica para o complexo formado com o $C_{3} b i s(4 C P)^{2+}$. A molécula é formada por dois anéis de piridínio, separados por uma ponte metilênica. Tratamos apenas o caso mais simples, onde a ponte metilênica é formada por uma cadeia de três átomos de carbono. Ainda assim, o problema agora é mais complexo em relação ao estudado na seção anterior, por causa da existência de diferentes conformações de equilíbrio para o complexo em solução.

Os resultados apresentados nesta seção seguem a ordem dos resultados apresentados na seção anterior. As geometrias dos complexos foram obtidas usando diferentes funcionais de troca e correlação, como B3LYP, CAM-B3LYP, M06-2X e wB97X-D. Como as estruturas dos complexos foram semelhantes, nos limitamos a analisar as estruturas obtidas com B3LYP. Novamente, o conjunto de funções base 6-311+G(d,p) foi usado para tratar os átomos de $\mathrm{H}, \mathrm{C}, \mathrm{N}$ do piridínio e o pseudopotencial aug-ccpVDZ-PP para o iodo.

\subsubsection{Estruturas de equilíbrio}

Partindo de diferentes conformações iniciais, foi possível identificar três estruturas estáveis distintas (Figura 4.6), denotadas por I-III. Em todas elas o anel se mostra planar e cada estrutura difere em relação aos ângulos de torção formados pelo nitrogênio do anel os três carbonos na ponte metilênica, chamados aqui de $\theta$ e $\phi$ (ver Figura 4.6.

A estrutura I é a única onde os dois ângulos diedrais são diferentes $\left(\theta=64^{\circ} \mathrm{e}\right.$ $\left.\phi=171^{\circ}\right)$. O $I^{-}$localiza-se sobre apenas um dos anéis em uma posição semelhante a observada no complexo da seção anterior. Na estrutura II, os dois anéis se fecham acomodando o iodeto entre eles, formando uma estrutura próxima a estrutura sanduíche proposta experimentalmente (ver Figura 1.4 da seção 1.2). Os dois ângulos são iguais a $\theta=\phi=87^{\circ}$. Já o na estrutura III, os dois anéis estão bastante afastados, os ângulos diedrais são iguais a $167^{\circ}$, de forma que a cadeia carbônica é bastante linear e o iodeto fica próximo ao plano dos anéis. 


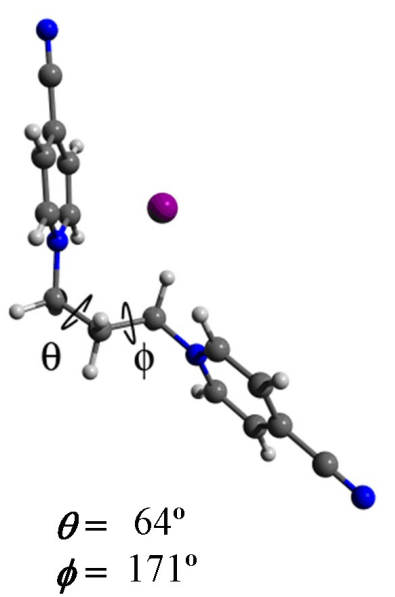

I

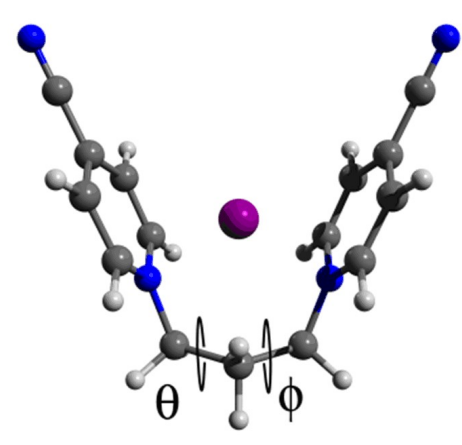

$\theta=\phi=87^{\circ}$

II

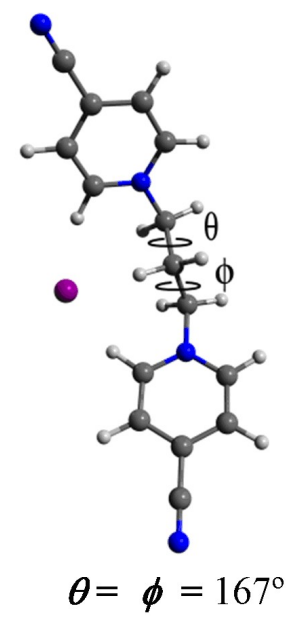

III

Figura 4.6: Representação das estruturas de mínima energia encontradas teoricamente, calculadas no nível de cálculo B3LYP. Bases 6-311+G(d,p) para C, N, H e aug-cc-PVDZ-PP para I.

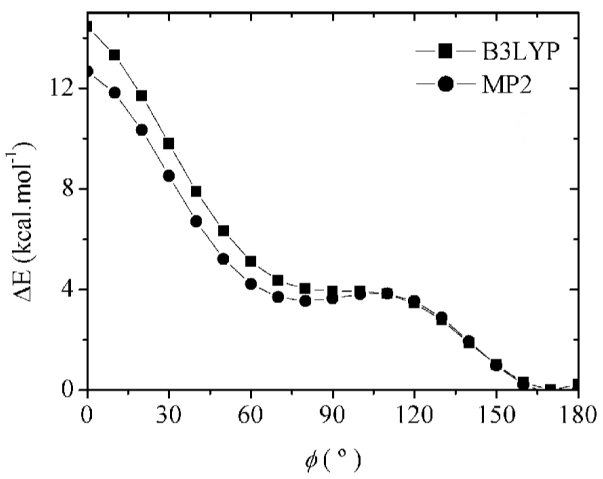

(a)

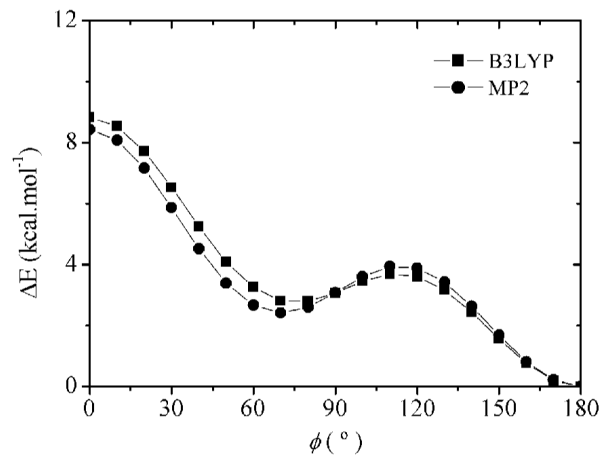

(b)

Figura 4.7: Varreduras do ângulo diedral do $C_{3} b i s(4 C P)^{2+}$, formado pelo nitrogênio no anel e os três carbonos da cadeia carbônica(ver Figura 4.6) : (a) mantendo o ângulo $\theta$ fixo em $71^{\circ}$. (b) mantendo o ângulo $\theta$ em $180^{\circ}$. Em cada ponto foi realizada uma otimização de geometria. Cálculos realizados com MP2 e B3LYP juntamente com bases 6-31G(d). 
Tabela 4.6: Propriedades eletrônicas dos complexos, calculadas usando B3LYP/6$311+\mathrm{G}(\mathrm{d}, \mathrm{p})(\mathrm{C}, \mathrm{H}, \mathrm{N}) /$ aug-cc-pVDZ-PP(I). $\Delta \mathrm{E}$ relativa à estrutura $\mathbf{I}\left(k_{c a l . m o l}{ }^{-1}\right)$. A energia eletrônica de interação foi calculada como a diferença entre a energia do complexo e a energia dos monômeros. O momento de dipolo (em Debye) foi calculado em relação ao centro de cargas nucleares.

\begin{tabular}{lcccccc}
\hline \hline & \multicolumn{4}{c}{ Vácuo } & \multicolumn{2}{c}{ Acetonitrila $(\mathrm{PCM})$} \\
\hline & I & II & III & I & II & III \\
\hline$\Delta E_{S C F}$ & 0.0 & 5.0 & 0.1 & 0.0 & 2.6 & -1.9 \\
$1 \Delta E_{\text {int }}$ & -160 & -162 & -157 & -5.0 & -5.4 & -6.2 \\
Dipolo & 14.7 & 15.2 & 14.1 & 26.5 & 27.8 & 22.8 \\
\hline \hline
\end{tabular}

A Figura 4.7 mostra como a energia do $C_{3} b i s(4 C P)^{2+}$ (sem o iodeto) varia com o ângulo $\phi$. O cálculo foi realizado nos níveis B3LYP e MP2, otimizando a geometria a cada passo. Na Figura 4.7a, o ângulo $\theta$ foi mantido fixo em $71^{\circ}$ durante todo o processo e os mínimos observados em cerca de $180^{\circ}$ e $90^{\circ}$ correspondem, respectivamente, a conformações semelhantes as conformações observadas nas estruturas I e II. Já na Figura $4.7 \mathrm{~b}$ o ângulo $\theta$ foi fixo em $180^{\circ}$ e os mínimos em $\phi=70^{\circ}$ e $\phi=180^{\circ}$ correspondem às estruturas I e III, respectivamente.

A Tabela 4.6 mostra algumas propriedades eletrônicas calculadas para as três estruturas do complexo. O cálculo da energia de interação sugere uma energia de ligação superior a $5 \mathrm{kcal} . \mathrm{mol}^{-1}$ em solução (Tabela 4.6), usando o modelo contínuo.

\subsubsection{Densidade de carga e análise de população}

Os mapas de diferença de densidade eletrônica entre o primeiro estado excitado e o estado fundamental para as estruturas I-III do complexo são mostrados na Figura 4.8. Novamente a transferência de carga característica (do iodeto para o anel) é observada.

É interessante notar que, na estrutura I, a densidade eletrônica do estado excitado localiza-se apenas sobre o anel onde o iodeto interage preferencialmente. Nas 

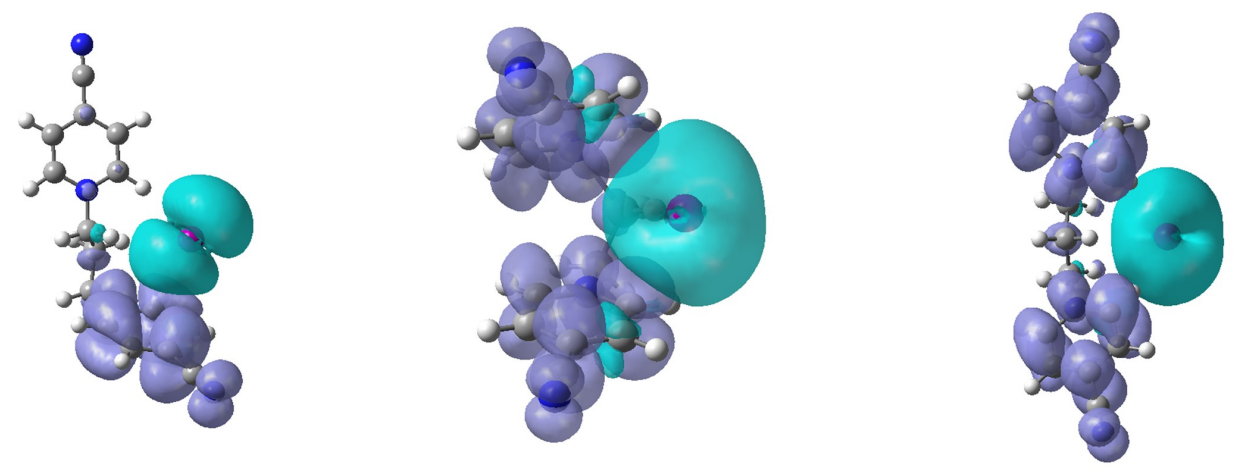

Figura 4.8: Mapas da diferença de densidade eletrônica entre o primeiro estado excitado e o estado fundamental do complexo $C_{3} b i s(4 C P)^{2+} I^{-}$. Calculado no nível CIS/3-21G. Em azul são as regiões que perdem carga na excitação e em roxo são as regiões para onde a carga se desloca.

estruturas II e III a carga é distribuída entre os dois anéis. As cargas naturais sobre os sítios atômicos foram calculadas usando no nível MP2 e bases 6-311+G(d,p) com pseudopotencial aug-cc-pVDZ-PP para o iodo. O resultado é apresentado na Figura 4.9 .

Novamente, a exemplo do complexo $C_{4}(4 C P)^{+} I^{-}$, uma transferência parcial da carga do iodeto para o anel ocorre já no estado fundamental (cerca de $17 \%$ a $28 \%$ da carga do iodeto, dependendo da estrutura, é transferida ao $\left.C_{3} b i s(4 C P)^{2+}\right)$. A carga de vácuo para o iodeto é de -0.763 , -0.715 e -0.832 nas estruturas I, II e III, respectivamente. A transferência é menos expressiva com a introdução do modelo contínuo, e a carga do iodeto em acetonitrila é de -0.866 em I, -0.849 em II e -0.898 em III.

A transferência de carga é maior quando o iodeto está acima do anel, como nas estruturas II e no anel inferior (ver Figura 4.9) da estrutura I. O nitrogênio desses anéis acaba com carga mais negativa (-0.303 em II e -0.313 em I) em relação aos nitrogênios do anel onde o iodeto se encontra no mesmo plano, como nas estruturas III (-0.265) e no anel superior da estrutura I (-0.264).

Os carbonos do anel ligados ao nitrogênio possuem cargas levemente positivas, com valores entre 0.050 e 0.106 . Como a carga dos hidrogênios ligados a esses carbonos está entre 0.228 e 0.279 , essa região do anel é bastante positiva, conferindo uma grande 

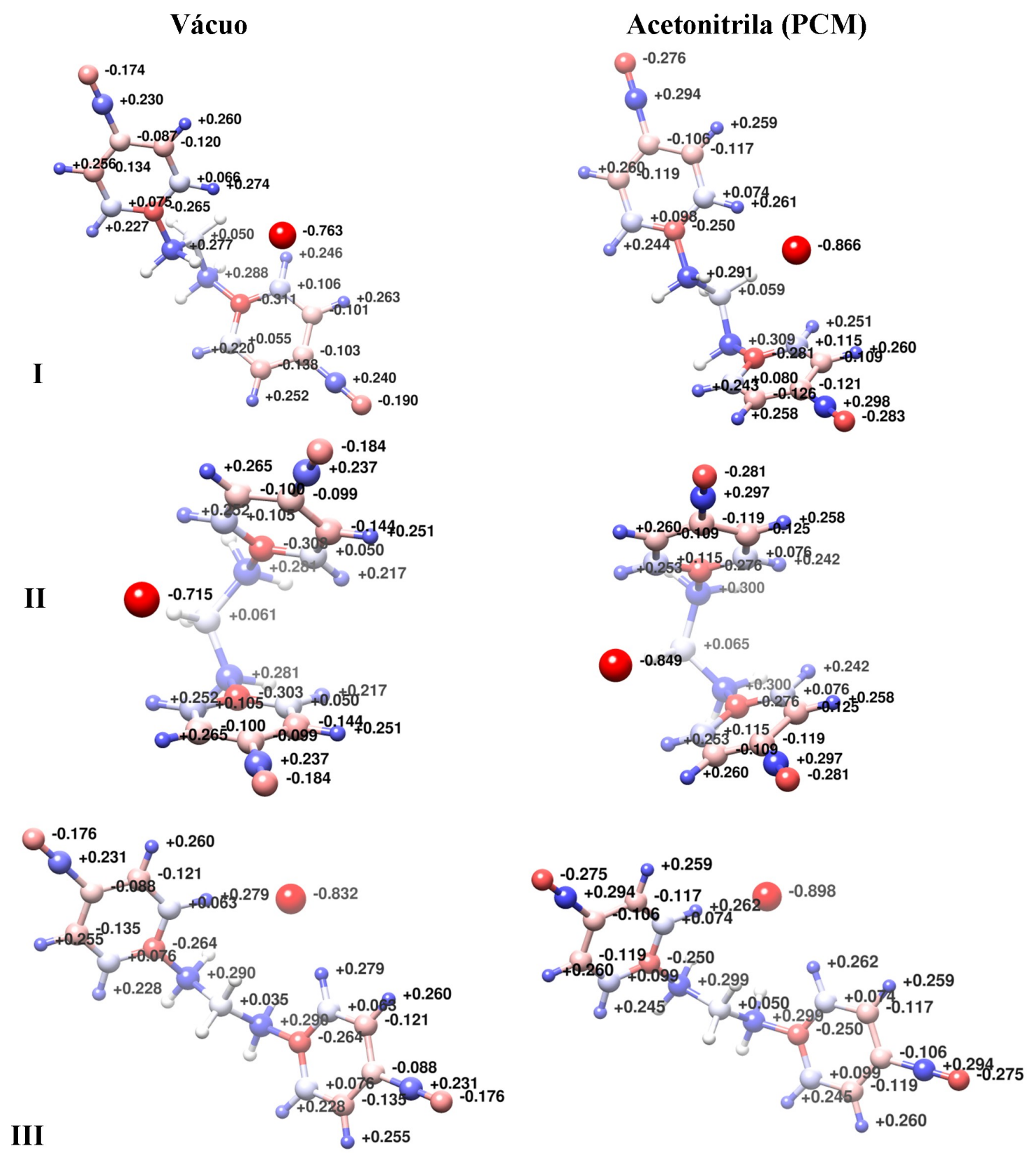

Figura 4.9: Cargas naturais para as estruturas I-III do complexo $C_{3} b i s(4 C P)^{2+} I^{-}$, em vácuo e em solução. Cálculos no nível MP2 (vácuo) e MP2-PCM (acetonitrila). Bases 6-311+G(d,p) para C, N ,H e aug-cc-PVDZ-PP para o iodo. 
interação eletrostática com o iodeto.

A distribuição de cargas calculadas em acetonitrila usando o modelo contínuo PCM sofre um processo de polarização, havendo deslocamento de carga para os nitrogênios do grupo nitrila, que passam a ser mais negativos, com cargas entre -0.275 e -0.281, em comparação com as cargas em vácuo, que estavam entre -0.174 e -0.184. Como resultado há um aumento do momento de dipolo calculado em relação ao centro de cargas, passando de $14.7 \mathrm{D}$ para $26.5 \mathrm{D}$ em I, de $15.2 \mathrm{D}$ para $27.8 \mathrm{D}$ em II e de 14.1 D para $22.8 \mathrm{D}$ em III.

\subsubsection{Energias de excitação}

A Tabela 4.7 mostra os comprimentos de onda de excitação das três transições de mais baixa energia. Foram calculados usando os funcionais com correção de longo alcance: LC-BLYP, LC- $\omega$ PBE, CAM-B3LYP e $\omega$ B97X-D. Como já visto no capítulo anterior, espera-se que sobretudo este dois últimos produzam resultados confiáveis para a energia de transição. A análise dos orbitais moleculares (Figura 4.10) mostram que a transição mais intensa é a HOMO $\rightarrow$ LUMO, observando também a transferência de carga, do orbital mais alto ocupado do iodeto para o orbital desocupado localizado sobre o anel aromático.

Novamente, CAM-B3LYP e $\omega$ B97X-D, associados ao modelo contínuo PCM, foram os funcionais com melhor desempenho quando comparados ao máximo da banda experimental em $400 \mathrm{~nm}$, medida em acetonitrila. Os valores calculados para a primeira transição em da estrutura I em acetonitrila (usando o modelo contínuo PCM) foram $410 \mathrm{~nm}$ (CAM-B3LYP) e 419 nm ( $\omega$ B97X-D), ambas com força de oscilador apreciável $(f=0.18)$. A mesma transição calculada para a estrutura III foi mais energética: 377 nm (CAM-B3LYP) e $352 \mathrm{~nm}(\omega \mathrm{B} 97 \mathrm{X}-\mathrm{D})$, com intensidades baixas $(f=0.02)$. Já a estrutura II apresentou uma transição menos energética, em 447 nm (CAM-B3LYP) e $432 \mathrm{~nm}$ ( $\omega \mathrm{B} 97 \mathrm{X}-\mathrm{D})$, com força de oscilador de 0.09 e 0.10 , respectivamente.

Embora a estrutura I apresente energias de excitação em acetonitrila em excelente concordância com o máximo da banda experimental, o resultado para as duas estruturas também estão dentro da banda de transferência de carga, que é bastante larga, se estendendo desde $330 \mathrm{~nm}$ até $600 \mathrm{~nm}$ aproximadamente. Apenas os cálculos 
Tabela 4.7: Comprimento de onda ( $\lambda$ em $\mathrm{nm})$ e respectivas força de oscilador $(f)$ para as três excitações eletrônicas de mais baixa energia, do complexo $C_{3} b i s(4 C P)^{2+} I^{-}$(I-III) usando diferentes métodos teóricos. Bases 6-311+G(d,p) para C, N ,H e aug-cc-PVDZ-PP para I

\begin{tabular}{|c|c|c|c|c|c|c|c|c|c|}
\hline \multirow[b]{2}{*}{ Estrutura } & \multirow[b]{2}{*}{ Transição } & \multicolumn{2}{|c|}{ LC-BLYP } & \multicolumn{2}{|c|}{$\mathrm{LC}-\omega \mathrm{PBE}$} & \multicolumn{2}{|c|}{ CAM-B3LYP } & \multicolumn{2}{|c|}{$\omega \mathrm{B} 97 \mathrm{X}-\mathrm{D}$} \\
\hline & & $\lambda$ & $f$ & $\lambda$ & $f$ & $\lambda$ & $f$ & $\lambda$ & $f$ \\
\hline \multicolumn{10}{|l|}{ Vácuo } \\
\hline \multirow[t]{3}{*}{$\mathbf{I}$} & 1 & 421 & 0.15 & 436 & 0.12 & 568 & 0.00 & 548 & 0.01 \\
\hline & 2 & 404 & 0.05 & 419 & 0.08 & 559 & 0.02 & 516 & 0.05 \\
\hline & 3 & 383 & 0.01 & 399 & 0.01 & 550 & 0.01 & 513 & 0.02 \\
\hline \multirow[t]{3}{*}{ II } & 1 & 447 & 0.13 & 461 & 0.14 & 575 & 0.07 & 560 & 0.08 \\
\hline & 2 & 419 & 0.03 & 435 & 0.02 & 575 & 0.00 & 559 & 0.00 \\
\hline & 3 & 406 & 0.01 & 423 & 0.00 & 545 & 0.01 & 532 & 0.01 \\
\hline \multirow[t]{3}{*}{ III } & 1 & 396 & 0.02 & 417 & 0.02 & 659 & 0.00 & 592 & 0.00 \\
\hline & 2 & 396 & 0.00 & 417 & 0.00 & 650 & 0.01 & 587 & 0.02 \\
\hline & 3 & 384 & 0.01 & 404 & 0.01 & 632 & 0.01 & 572 & 0.01 \\
\hline \multicolumn{10}{|c|}{ Acetonitrila (PCM) } \\
\hline \multirow[t]{3}{*}{$\mathbf{I}$} & 1 & 338 & 0.22 & 348 & 0.22 & 410 & 0.18 & 410 & 0.18 \\
\hline & 2 & 300 & 0.00 & 310 & 0.00 & 401 & 0.02 & 389 & 0.02 \\
\hline & 3 & 297 & 0.00 & 308 & 0.00 & 397 & 0.00 & 385 & 0.00 \\
\hline \multirow[t]{3}{*}{ II } & 1 & 346 & 0.10 & 356 & 0.11 & 447 & 0.09 & 432 & 0.10 \\
\hline & 2 & 323 & 0.23 & 333 & 0.24 & 420 & 0.04 & 406 & 0.05 \\
\hline & 3 & 304 & 0.01 & 314 & 0.04 & 411 & 0.00 & 396 & 0.00 \\
\hline \multirow[t]{3}{*}{ III } & 1 & 270 & 0.04 & 279 & 0.04 & 377 & 0.02 & 352 & 0.02 \\
\hline & 2 & 269 & 0.01 & 278 & 0.01 & 375 & 0.01 & 350 & 0.01 \\
\hline & 3 & 261 & 0.01 & 269 & 0.01 & 369 & 0.01 & 343 & 0.01 \\
\hline
\end{tabular}




\section{LUMO}
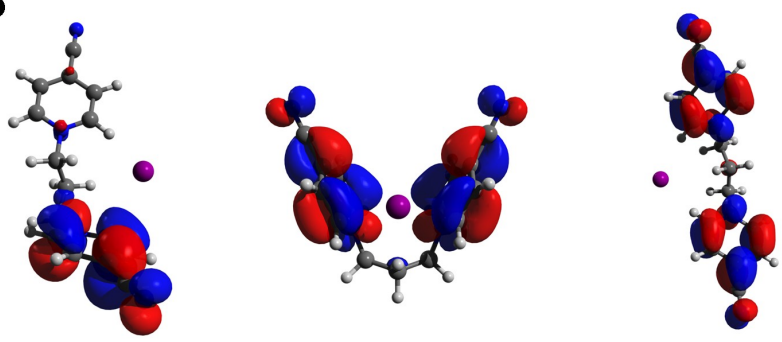

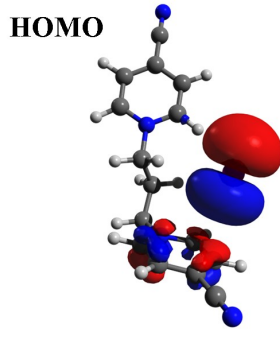

I

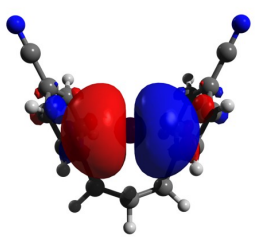

II

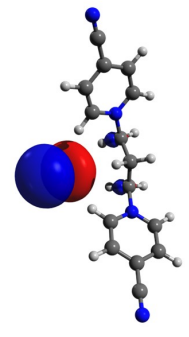

III

Figura 4.10: Representação dos orbitais de fronteira HOMO (abaixo) e LUMO (acima) para as estruturas I-III do complexo $C_{3} b i s(4 C P)^{2+} I^{-}$, obtidos a partir da densidade eletrônica do cálculo no nível HF e bases 6-311+G(d,p) para C, H, N e aug-cc-pVDZ para I

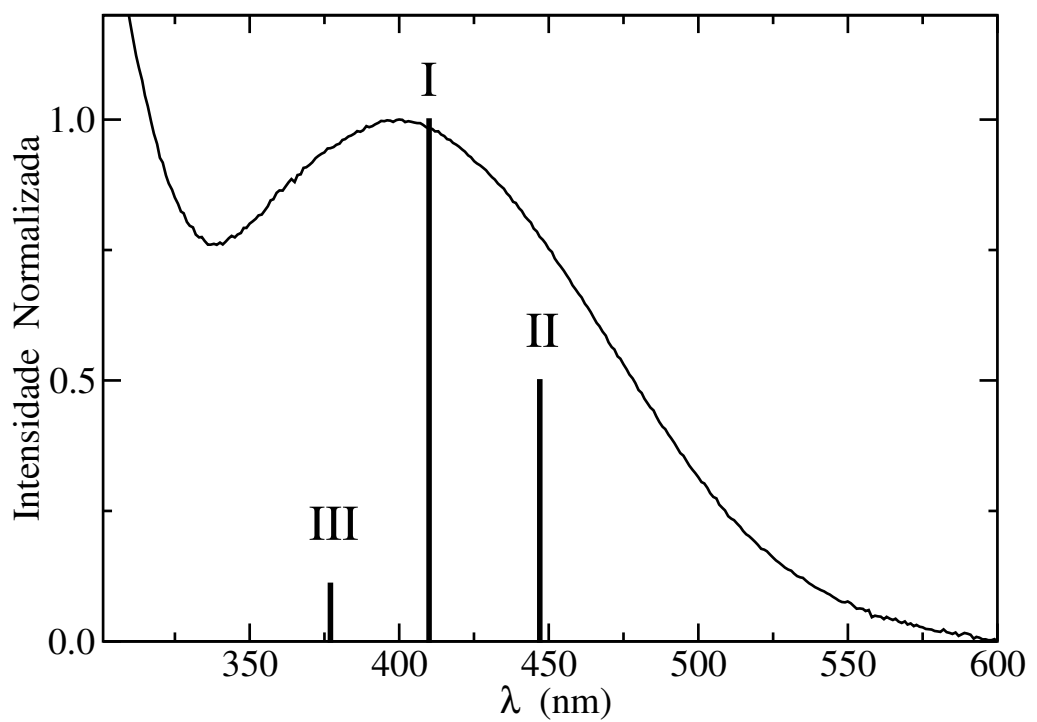

Figura 4.11: Comparação entre as energias de excitação calculadas (linhas verticais) e o espectro experimental (linha curva) do $C_{3} b i s(4 C P)^{2+} I^{-}$. As linhas verticais correspondem as excitação de maior intensidade nas estruturas I-III, calculadas em acetonitrila usando o modelo contínuo PCM no nível de cálculo CAM-B3LYP e bases 6-311+G(d,p) para C, H, N e aug-cc-pVDZ para I. As intensidades foram ponderadas pelas respectivas força de oscilador. 
de energia e do espectro em estruturas de mínima energia, portanto, não são conclusivos em relação à estrutura do complexo, indicando que muito possivelmente, todas as estruturas são acessíveis em solução.

\subsubsection{Adição de um segundo iodeto}

Nesta seção estudamos estruturas adicionais formadas pelo $C_{3} b i s(4 C P)^{2+}$ associado a dois contra-íons $I^{-}$, resultando em um complexo molecular neutro. As estruturas encontradas, chamadas de IV e V, são apresentadas na Figura 4.12.

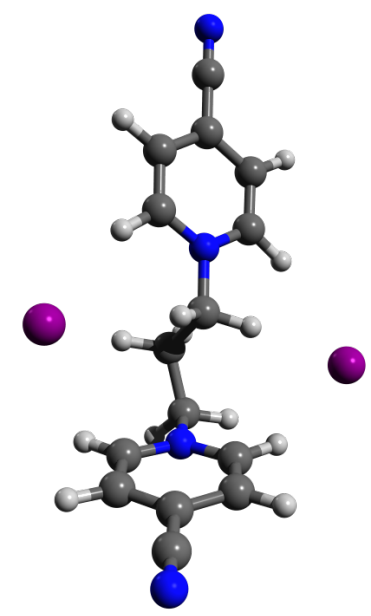

IV

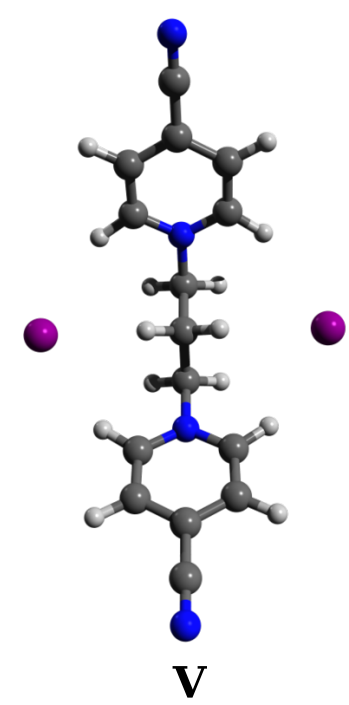

Figura 4.12: Representação estrutural da geometrias otimizadas dos complexo formado pelo $C_{3}$ bis $(4 C P)^{2+}$ com dois iodetos. Funcional B3LYP. Bases $6-311+\mathrm{G}(\mathrm{d}, \mathrm{p})$ para $\mathrm{C}, \mathrm{N}, \mathrm{H}$ e aug-cc-PVDZ-PP para I.

As geometrias foram otimizadas usando o funcional B3LYP e o conjunto de funções bases 6-311+G(d,p) nos átomos de H,C e N e o pseudopotencial aug-cc-pVDZPP para o I. A estrutura IV foi obtida adicionando um segundo $I^{-}$a estrutura I. Esta conformação é interessante, pois permite que apenas um dos átomos de iodo fique acima de um dos anéis, o que favorece a transferência, enquanto que o outro iodeto interage apenas com os hidrogênios.

Já a estrutura V foi obtida adicionado o segundo na estrutura III. Os dois iodetos se posicionam de forma simétrica aos dois anéis, interagindo apenas com os hidrogênios 
Tabela 4.8: Comprimento de onda (em nm) e respectivas força de oscilador (entre parênteses) para as seis excitações eletrônicas de mais baixa energia do complexo $C_{3}$ bis $(4 C P)^{2+}+2 I^{-}$ (estruturas IV e V). Bases 6-311+G(d,p) para C, N ,H e aug-cc-PVDZ-PP para I

\begin{tabular}{llllc}
\hline \hline & \multicolumn{2}{c}{ CAM-B3LYP } & \multicolumn{2}{c}{$\omega$ B97X-D } \\
& IV & V & IV & V \\
\hline 1 & Vácuo & & & \\
2 & $607(0.01)$ & $666(0.01)$ & $548(0.00)$ & $590(0.01)$ \\
3 & $599(0.00)$ & $640(0.01)$ & $543(0.01)$ & $564(0.00)$ \\
4 & $574(0.01)$ & $635(0.00)$ & $531(0.00)$ & $564(0.01)$ \\
5 & $567(0.00)$ & $623(0.00)$ & $519(0.06)$ & $552(0.00)$ \\
6 & $545(0.04)$ & $605(0.00)$ & $506(0.00)$ & $536(0.00)$ \\
\hline & $532(0.01)$ & $601(0.00)$ & $497(0.08)$ & $535(0.00)$ \\
\hline 1 & Acetonitrila (PCM) & & & $354(0.01)$ \\
2 & $404(0.14)$ & $383(0.01)$ & $389(0.15)$ & $346(0.00)$ \\
3 & $392(0.00)$ & $373(0.00)$ & $374(0.00)$ & $338(0.01)$ \\
4 & $385(0.01)$ & $367(0.01)$ & $368(0.01)$ & $338(0.00)$ \\
5 & $367(0.01)$ & $365(0.00)$ & $342(0.01)$ & $337(0.00)$ \\
6 & $361(0.00)$ & $364(0.00)$ & $334(0.00)$ & $333(0.00)$ \\
\hline \hline
\end{tabular}

tanto da cadeia quanto dos anéis. Os valores para o comprimento de onda de excitação das seis transições de mais baixa energia para as estruturas IV e $\mathbf{V}$ são apresentados na Tabela 4.8 .

Todas as transições calculadas para os complexos isolados (vácuo) têm intensidade quase nulas. Quando se inclui o efeito do solvente com o modelo contínuo PCM, apenas a primeira transição da estrutura IV ganha intensidade. Esta transição corresponde a uma transição $\mathrm{HOMO} \rightarrow \mathrm{LUMO}+1$. Como pode ser observado na Figura 4.13, o orbital HOMO está localizado predominantemente sobre o átomo de iodo acima do anel e o orbital LUMO+1 está localizado predominantemente sobre o anel abaixo 


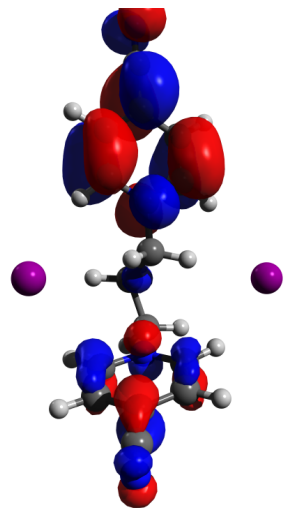

(a) LUMO

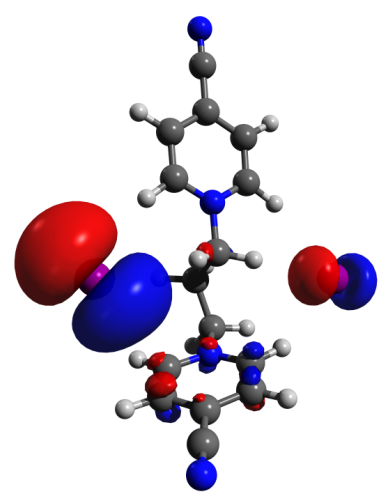

(d) HOMO

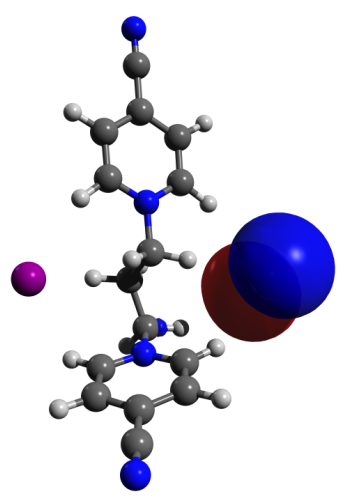

(g) HOMO-3

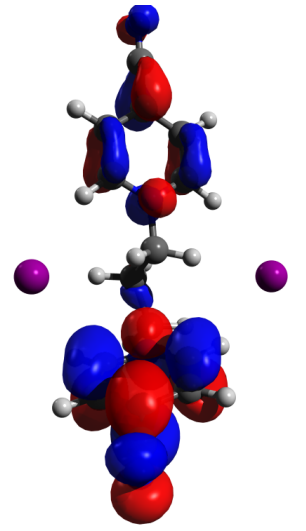

(b) $\mathrm{LUMO}+1$

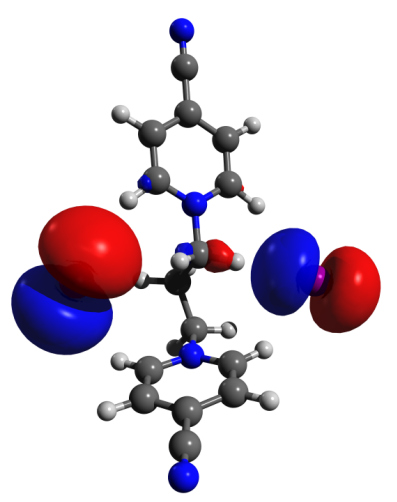

(e) HOMO-1

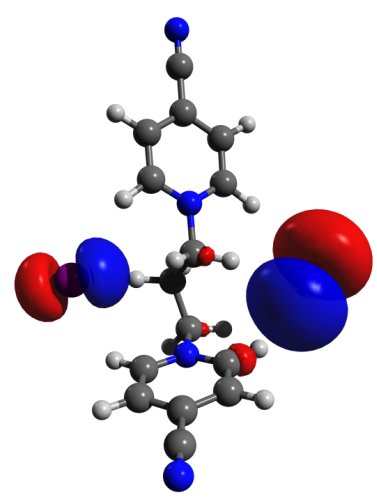

(h) HOMO-4

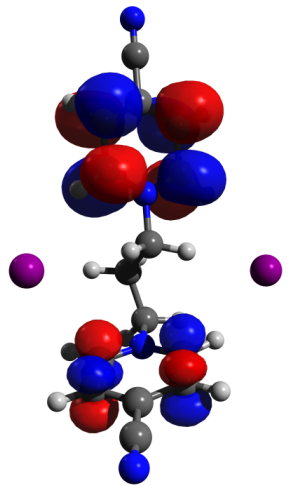

(c) $\mathrm{LUMO}+2$

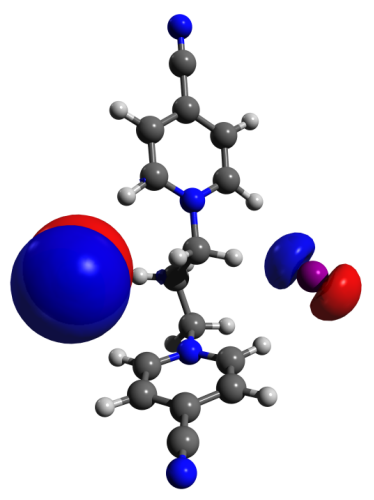

(f) HOMO-2

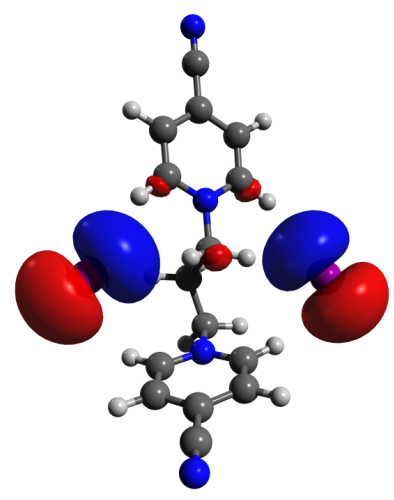

(i) HOMO-5

Figura 4.13: Orbitais Moleculares de KS para o complexo formado pelo $C_{3}$ bis $(4 C P)^{2+}$ com dois $I^{-}$, calculados com o funcional CAM-B3LYP. Bases 6-311+G(d,p) e aug-cc-pVDZ$\mathrm{PP}(\mathrm{I})$. 
deste. O valor da excitação foi de $404 \mathrm{~nm}(f=0.14)$ para o funcional CAM-B3LYP e de $389 \mathrm{~nm}(f=0.15)$ para o funcional $\omega$ B97X-D, ambos próximos ao valor obtido para a estrutura I. De forma geral não são observadas grandes modificações nos valores das excitações, ou seja, o segundo $I^{-}$não afeta o espectro de absorção.

Os resultados desta seção e da seção anterior deixam claros, portanto, que apenas a medida do espectro eletrônico de absorção não é suficiente para caracterizar as estruturas do complexo de $I^{-}$com o $C_{3} b i s(4 C P)^{2+}$. Ao contrário do foi proposto experimentalmente, não existe apenas uma estrutura possível, mas pelo mesmos três conformações para o $C_{3}$ bis $(4 C P)^{2+}$ em que se observam a formação de complexos moleculares. Também é importante ressaltar que os complexos podem ser formados pela associação de um ou dois $I^{-}$.

A estrutura II era semelhante a estrutura sanduíche proposta experimentalmente, com o dois anéis se fechando sobre o contra-íon. Além de ser uma estrutura menos favorável energeticamente em relação as demais (ainda que tenham sido resultados baseados em geometrias de vácuo) a excitação calculada para ela também aparece cerca de $30 \mathrm{~nm}$ deslocada para o vermelho (maiores comprimentos de onda quando) quando comparado ao resultado experimental.

A estrutura $\mathbf{I}$ foi a que apresentou resultados mais consistentes com as medidas experimentais. Ela apresenta uma excitação de transferência de carga intensa em 410 nm, muito próximo ao máximo da banda experimental. As excitações desta estrutura são muito próximas das excitações observadas nos complexos formados pelo $C_{4}(4 C P)^{+}$.

Mas vimos também que o iodeto pode se associar ao $C_{3} b i s(4 C P)^{2+}$ na região próxima ao plano do anel, formando complexos estáveis mas onde não são observados transferência eletrônica devido a absorção de fótons. Isto ocorre nas estruturas $\mathbf{I}, \mathbf{V}$ e com uma dos iodetos da estruturas IV.

A estrutura IV é interessante pois o $C_{3} b i s(4 C P)^{2+}$ está associado a dois $I^{-}$, mas apenas um dos contra-íons participa da transferência de carga, o que explica porque a medida experimental apresentava a relação estequiométrica equimolar (1:1). No próximo capítulo, apresentamos resultados mais aprofundado do comportamento dos complexos em solução, obtidos com a dinâmica molecular. 


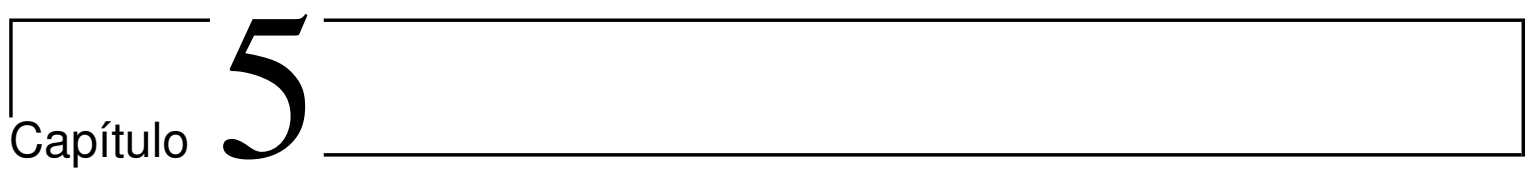

\section{Dinâmica molecular dos complexos}

Neste capítulo vamos além das estruturas de mínima energia e buscamos uma descrição mais realista e aprofundada dos complexos em solução.

O início do capítulo é dedicado aos resultados obtidos usando a dinâmica ab initio de Born-Oppenheimer. A dinâmica foi realizada apenas na fase gasosa, pois a inclusão de moléculas explícitas de acetonitrila torna a dinâmica inviável por causa do alto custo computacional.

A dinâmica dos complexos em solução foi possivel somente usando a dinâmica molecular clássica. As informações da dinâmica por primeiros princípios foram usadas como referência para refinar os parâmetros do campo de força.

Finalmente, apresentamos os resultados da dinâmica molecular dos complexos em solução e o cálculo de espectro de absorção. 


\subsection{Dinâmica molecular por primeiros princípios}

\subsubsection{Detalhes computacionais}

As dinâmicas de Born-Oppenheimer (BOMD) dos pares iônicos formados pelo iodeto com $C_{4}(4 C P)^{+}$e $C_{3} b i s(4 C P)^{2+}$ foram realizadas no ensemble NVT, em uma caixa cúbica de largura $20 \AA$. Utilizamos o formalismo GAPW [251] implementado no programa CP2K [191]. Foram usadas como funções bases gaussianas o conjunto DZVP-MOLOPT-SR-GTH [252] juntamente com os pseudopotenciais de GoedeckerTeter-Hutter (GTH) com um raio de corte de 280 Ry. Para melhor performance, os cálculos foram realizados usando o funcional PBE para o cálculo das forças. Este nível de cálculo tem sido usado com êxito na descrição de clusters moleculares [253-255]. O passo de integração foi de 0.25 fs e o tempo total de simulação foi de 50 ps (200000 passos). A simulação foi realizada usando o termostato estocástico de rescalonamento de velocidades (chamado de CSRV no CP2K), com uma temperatura alvo de $300 \mathrm{~K}$ e constante de acoplamento de 5.0 fs. O arquivo de entrada para esta dinâmica pode ser encontrado no Apêndice D.1.

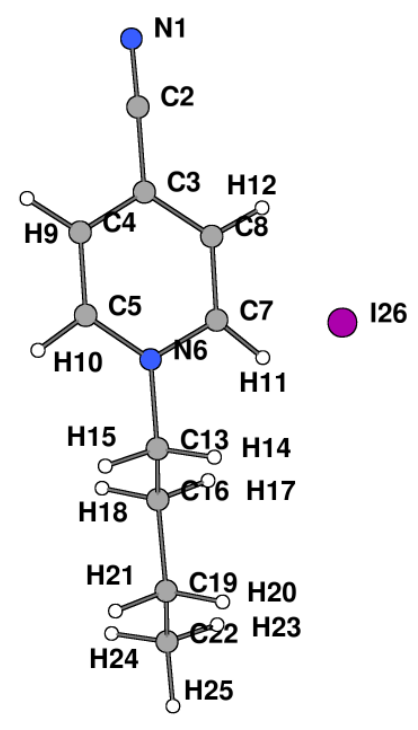

(a)

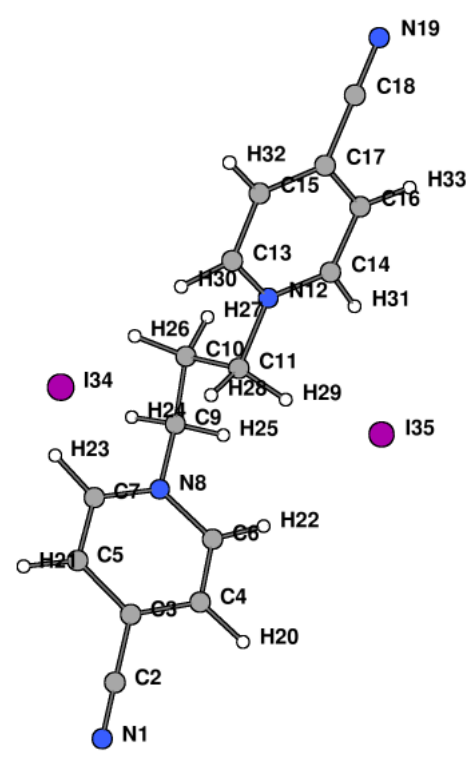

(b)

Figura 5.1: Representação da estrutura dos complexos e numeração atômica adotada: (a) $C_{4}(4 C P)^{+} I^{-}$e (b) $C_{3} b i s(4 C P)^{2+} 2 I^{-}$ 
Foram usadas como configurações iniciais da dinâmica as geometrias otimizadas para os complexos $C_{4}(4 C P)^{+} I^{-}$e $C_{3} b i s(4 C P)^{2+}$ com dois $I^{-}$. Para este último usamos a estrutura IV que, como visto na seção 4.2.4, apresenta excitações de transferência de carga em boa concordância com o resultados experimental. As geometrias dos complexos com a numeração atômica adotada são mostradas na Figura 5.1.

\subsubsection{Dinâmica molecular do complexo em vácuo}

A evolução temporal da temperatura e energia potencial durante a dinâmica dos complexos é mostrada nos gráficos da Figura 5.2. Para o cálculo das propriedades foram desconsiderados os primeiros 10 ps da dinâmica que, como se observa nesta figura, já são suficientes para que o sistema esteja em equilíbrio, com as grandezas oscilando ao redor do valor médio.

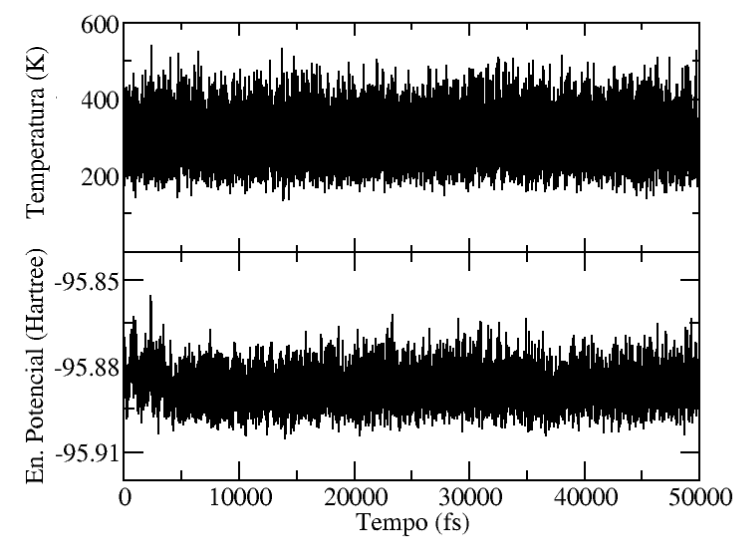

(a)

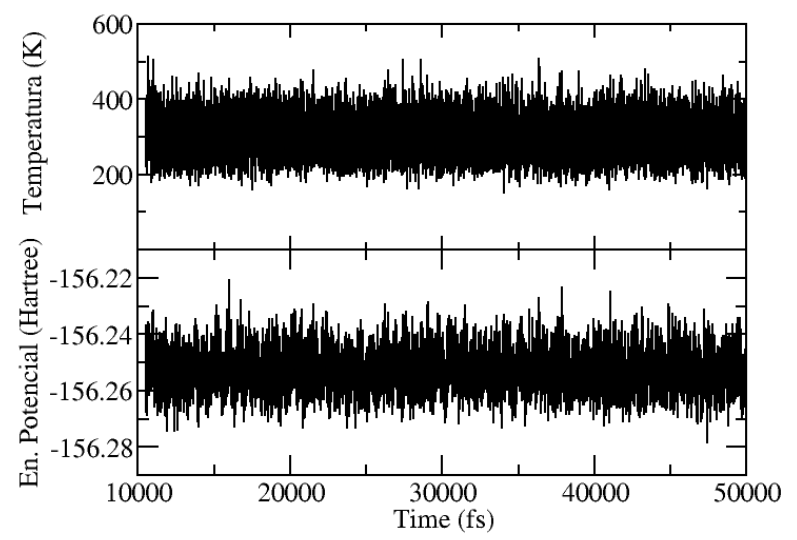

(b)

Figura 5.2: Evolução temporal da temperatura e energia potencial durante a dinâmica molecular para os complexos: (a) $C_{4}(4 C P)^{+} I^{-}$e (b) $C_{3} b i s(4 C P)^{2+} 2 I^{-}$

Não foram observadas grandes mudanças conformacionais ao longo da dinâmica. Para os dois sistema, apenas observa-se que o iodeto flutua ao redor da posição de equilíbrio, como é mostrado na Figura 5.3. Uma das razões é a alta interação eletrostática, já que trata-se de uma dinâmica que não inclui o efeito do solvente. O tempo de 


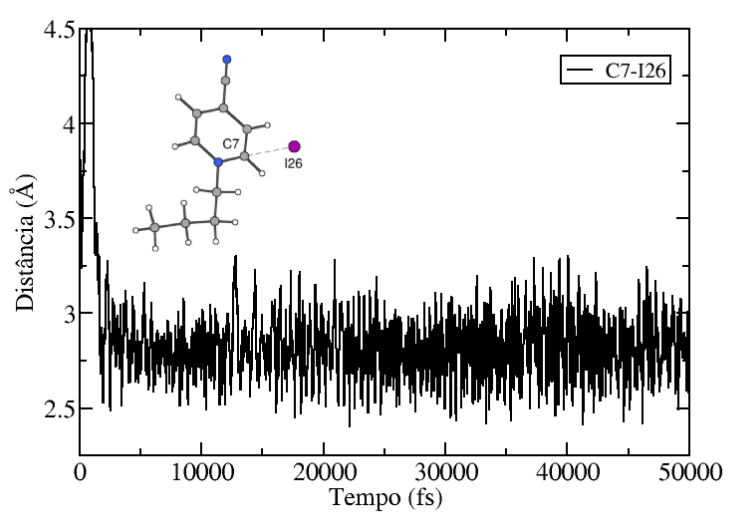

(a)

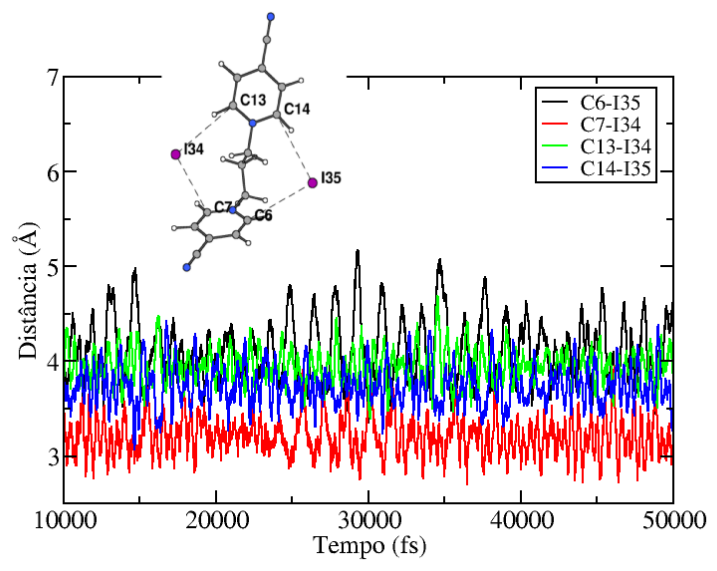

(b)

Figura 5.3: Evolução da distância C … I ao longo da dinâmica molecular molecular de BornOppenheimer: (a) $C_{4}(4 C P)^{+} I^{-}$e (b) $C_{3} b i s(4 C P)^{2+} 2 I^{-}$

simulação, embora suficiente para amostrar as propriedades da conformação adotada, pode não ser suficiente para permitir a mudança conformacional.

Nos dois complexos a distância o iodeto fica próximo do carbono $C 7$ durante toda a dinâmica, sugerindo uma forte interação neste sítio. A distância média foi de $2.86 \pm 027 \AA$ no complexo com $C_{4}(4 C P)^{+}$e de $3.20 \pm 0.20 \AA$ no complexo com $C_{3} b i s(4 C P)^{2+}$, um pouco maior do que a obtida nas geometrias otimizadas $(2.59 \AA \mathrm{e}$ $2.93 \AA$, respectivamente).

A Figura 5.4 mostra a distribuição de modos vibracionais associados a interação do iodeto com o carbono. Os modos vibracionais foram calculados usando a transformada de Fourier da função de autocorrelação de velocidades, $C_{A A}(t)$, onde a velocidade consiste na taxa de variação da distância $C-I$, dada por

$$
A=\frac{d x}{d t}
$$

onde $x=r(C-I)$ representa a distância do iodeto ao carbono mais próximo no instante t. Esse tipo de abordagem foi usada recentemente para caracterizar a interação de íons em clorofilas e nanoestruturas [253, 254]. Maiores detalhes de como os modos vibracionais foram calculados neste trabalho podem ser encontrados no Apêndice B. 


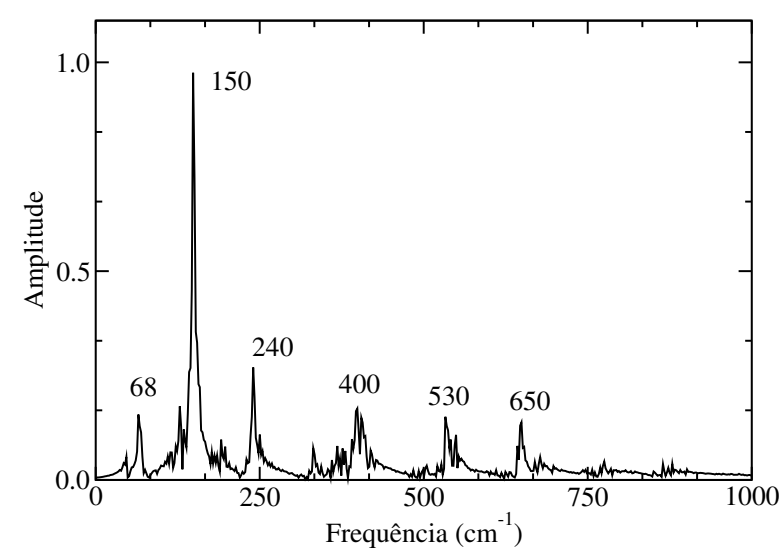

(a)

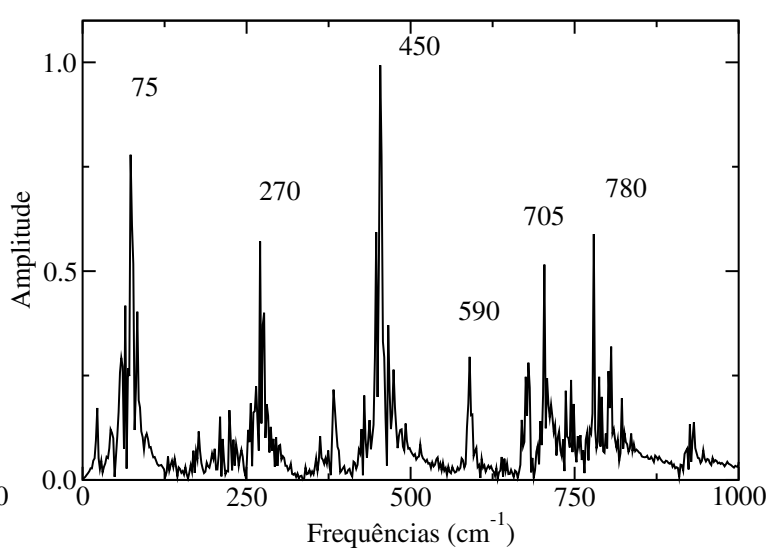

(b)

Figura 5.4: Transformada de Fourier da Função de autocorrelação de velocidades (a) $C_{4}(4 C P)^{+} I^{-}$e (b) $C_{3} b i s(4 C P)^{2+} 2 I^{-}$

Tabela 5.1: Modos vibracionais calculados na BOMD e calculados usando as geometrias otimizadas (OPT). As intensidades calculadas são apresentada entre parênteses.

\begin{tabular}{cccc}
\hline \hline & & \multicolumn{2}{c}{$C_{3} b i s(4 C P)^{2+}+2 I^{-}$} \\
BOMD & OPT & BOMD & OPT \\
\hline 68 & $72(12)$ & 44 & $49(11)$ \\
150 & $128(16)$ & 66 & $66(12)$ \\
240 & $277(8)$ & 75 & $76(16)$ \\
400 & $411(16)$ & & $120(28)$ \\
530 & $567(68)$ & & $143(15)$ \\
650 & $700(16)$ & 270 & $290(4)$ \\
& & 450 & $566(13)$ \\
& & 590 & $585(23)$ \\
& & 705 & $713(14)$ \\
& & 747 & $750(15)$ \\
& & 780 & $796(21)$ \\
& & 807 & $806(25)$ \\
\hline \hline
\end{tabular}


A Tabela 5.1 mostra os modos vibracionais mais intensos entre 0 e $800 \mathrm{~cm}^{-1}$ calculados para a geometria otimizada dos complexos, comparados com os modos vibracionais calculados a partir da BOMD. Nesta região aparecem as vibrações relacionadas aos deslocamentos entre o iodeto e o anel.

No caso do $C_{4}(4 C P)^{+} I^{-}$todos os principais picos que aparecem no gráfico da Figura 5.4a podem ser associados a frequências vibracionais da estrutura de mínima energia. Observando os modos vibracionais para a estrutura otimizada verifica-se que o pico mais intenso que aparece em $150 \mathrm{~cm}^{-1}$ caracteriza o movimento de estiramento entre o iodeto e o carbono do anel. O valor é baixo se comparado ao valor para o estiramento $C-I$ em uma ligação covalente, que é de $450-500 \mathrm{~cm}^{-1}$ [256,257], já que nos complexos iodo não forma uma ligação covalente com o carbono do anel.

Já o caso do $C_{3} b i s(4 C P)^{2+}$ é mais complexo uma vez que há um número maior de átomos envolvidos e não existe apenas um único pico intenso associado. Na Figura 5.4b fica caracterizada a interação apenas do I34 com o anel, mas ainda assim a maioria dos picos podem ser associados também com as frequências vibracionais da estrutura de mínima energia, com exceção do pico em $450 \mathrm{~cm}^{-1}$, que não aparece com intensidade na estrutura otimizada.

\subsubsection{Espectro de absorção calculado}

Para a análise da banda de transferência de carga, foram amostradas 100 configurações de cada complexo nos últimos 40 ps de dinâmica (separadas por intervalos de 0.40 ps). Estas configurações aparecem superpostas na Figura 5.5. Cada espectro foi calculado convoluindo funções lorentzianas aos resultados dos cálculos das energias de excitação, usando uma largura de linha estreita de $0.1 \mathrm{eV}$. Assim o alargamento observado na banda é proveniente principalmente dos efeitos de térmicos e do efeito do solvente (quando incluído no cálculo).

Vamos analisar inicialmente os resultados obtidos para o $C_{4}(4 C P)^{+} I^{-}$isolado (sem incluir o solvente). Em cada uma das configurações foram calculadas as 6 excitações de mais baixa energia que, como foi visto no capítulo anterior, são responsáveis pelas duas bandas de transferência de carga que aparecem em solventes de baixa polaridade. O resultado para o espectro calculado é apresentado na Figura 5.6. 


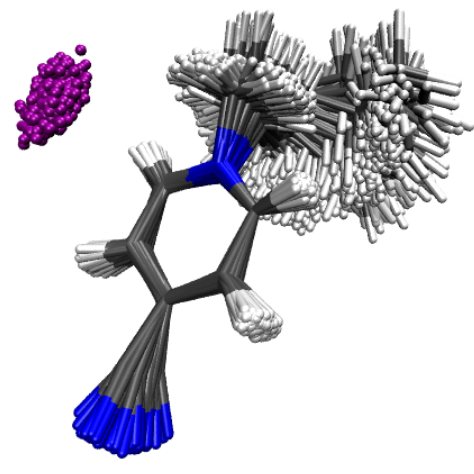

(a)

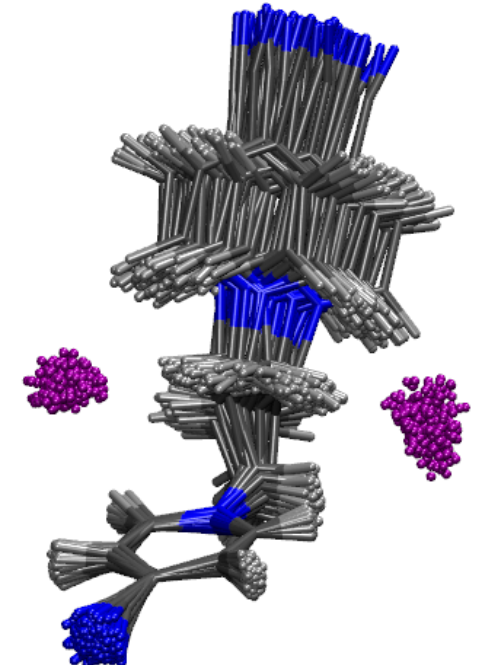

(b)

Figura 5.5: Superposição de estruturas em 100 configurações amostradas uniformemente entre 10 ps e 50 ps: (a) $C_{4}(4 C P)^{+} I^{-}$e (b) $C_{3} b i s(4 C P)^{2+} 2 I^{-}$

Observa-se no espectro de vácuo o aparecimento das duas bandas característica, com máximos em $330 \mathrm{~nm}$ e $448 \mathrm{~nm}$. A diferença entre as bandas, de $118 \mathrm{~nm}$ está em bom acordo com a diferença de $150 \mathrm{~nm}$ reportada experimentalmente em diclorometano [248]. A diferença de $32 \mathrm{~nm}$ em relação ao resultado experimental foi devido a banda de maior comprimento de onda, que é bastante larga, com excitações que se estendem de $400 \mathrm{~nm}$ a $600 \mathrm{~nm}$ e com o máximo em $448 \mathrm{~nm}$. Experimentalmente o resultado para máximo é de $480 \mathrm{~nm}$ (diclorometano) [248], $495 \mathrm{~nm}$ (clorofórmio) e $529 \mathrm{~nm}$ (benzeno) [246]. A principal contribuição para essa banda vem da $3^{a}$ excitação, do tipo $\mathrm{HOMO} \rightarrow$ LUMO. As excitações que contribuem para banda de menor comprimento de onda são do tipo $\mathrm{HOMO} \rightarrow \mathrm{LUMO}+1$, no caso da excitação 4, e HOMO-1 $\rightarrow$ LUMO+1 e HOMO-2 $\rightarrow$ LUMO+1, no caso da excitação 6.

Observa-se ainda nos cálculos de vácuo uma dependência linear entre o comprimento de onda de excitação e a distância $C-I$ entre o iodeto e carbono $C 7$ do anel aromático, conforme mostrado na Figura 5.7a. Note que sobretudo as duas primeiras excitações aparecem com comprimentos de onda acima de $650 \mathrm{~nm}$ para distâncias maiores do que $3.0 \AA$. Isto poder estar relacionado a deficiências dos funcionais de troca e 


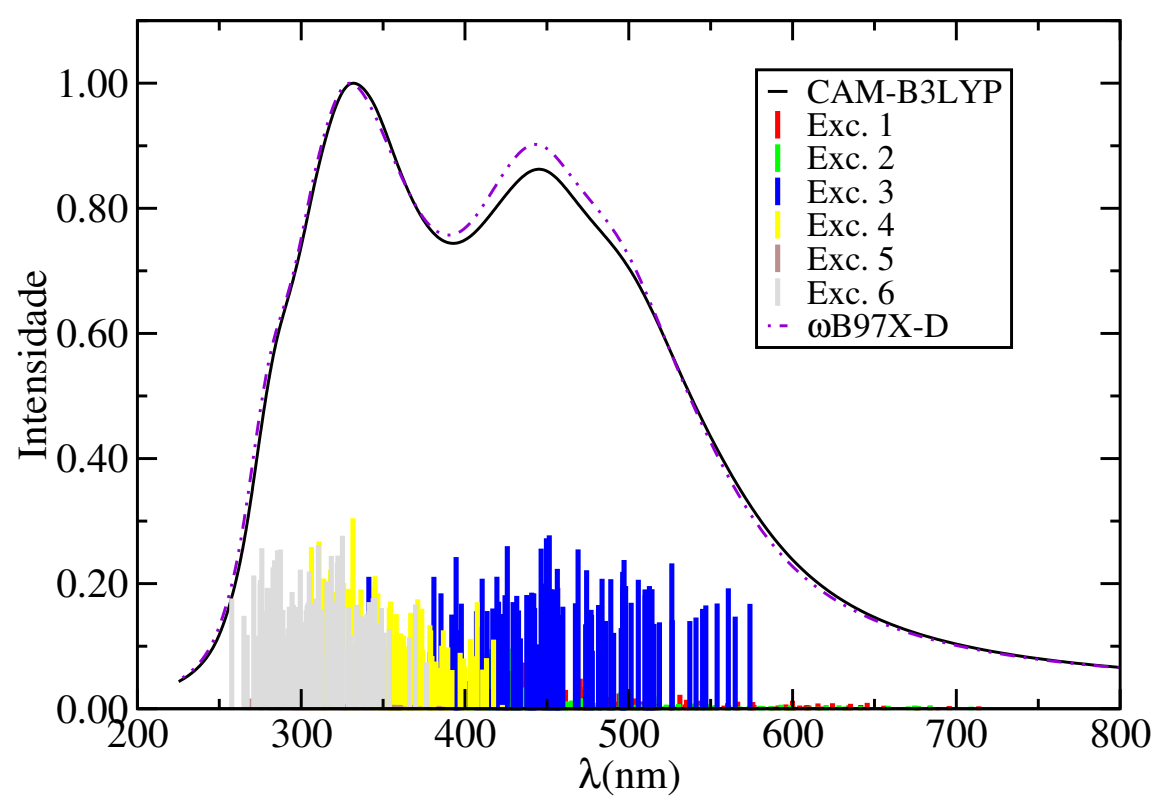

Figura 5.6: Espectro calculado para o complexo $C_{4}(4 C P)^{+} I^{-}$a partir de 100 configurações amostradas da dinâmica de Born-Oppenheimer. As excitações verticais de cada configuração são apresentadas também, onde a intensidade é dada pela respectiva força de oscilador.

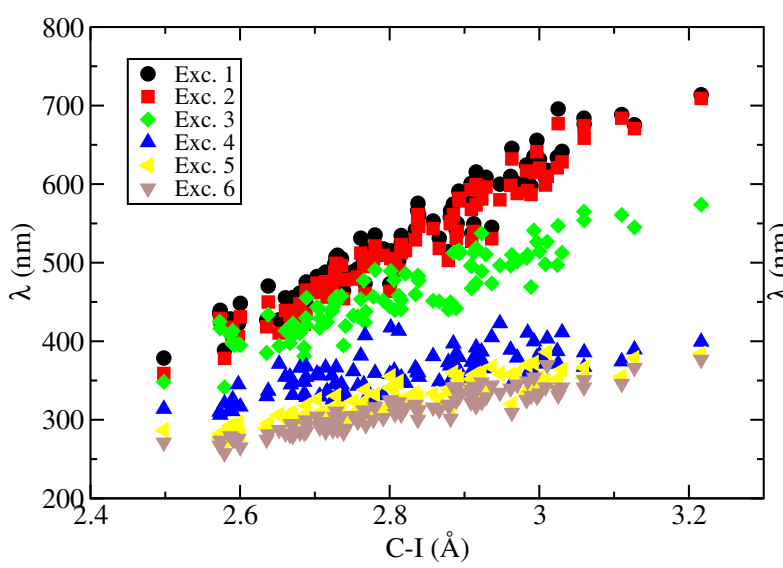

(a)

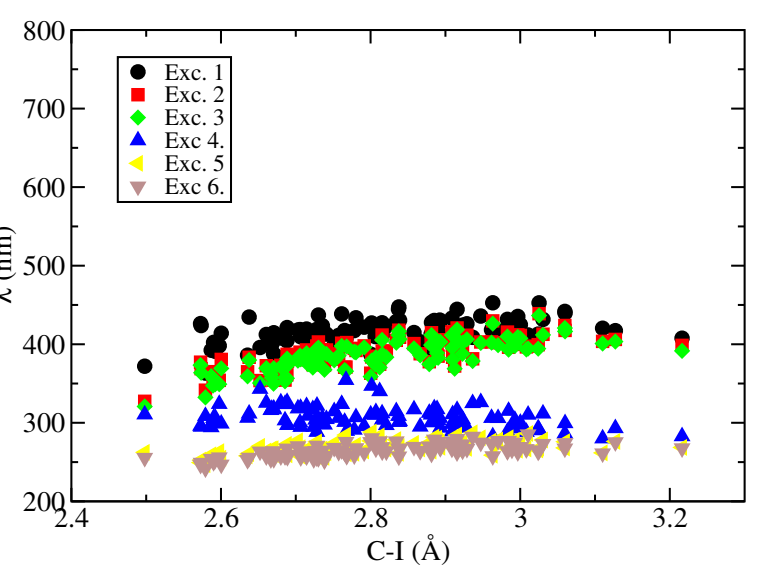

(b)

Figura 5.7: Relação entre as energias de excitação calculadas em função da distância do I ao carbono do anel no $C_{4}(4 C P)^{+} I^{-}$: (a) vácuo (b) acetonitrila (PCM) 


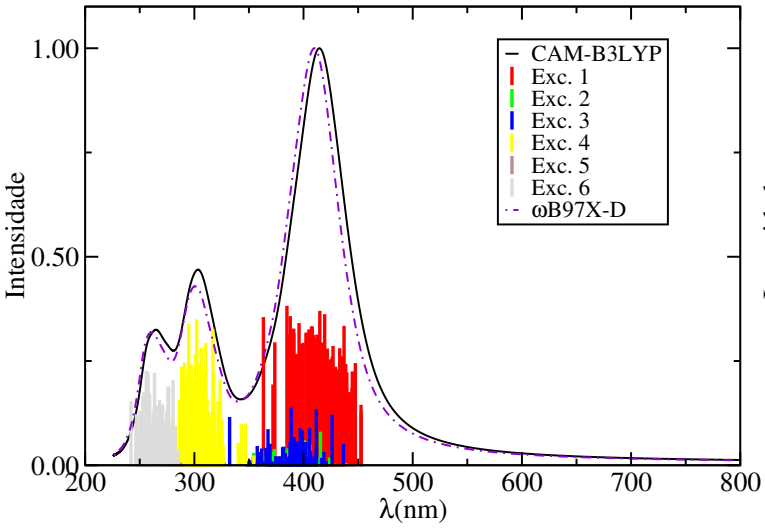

(a)

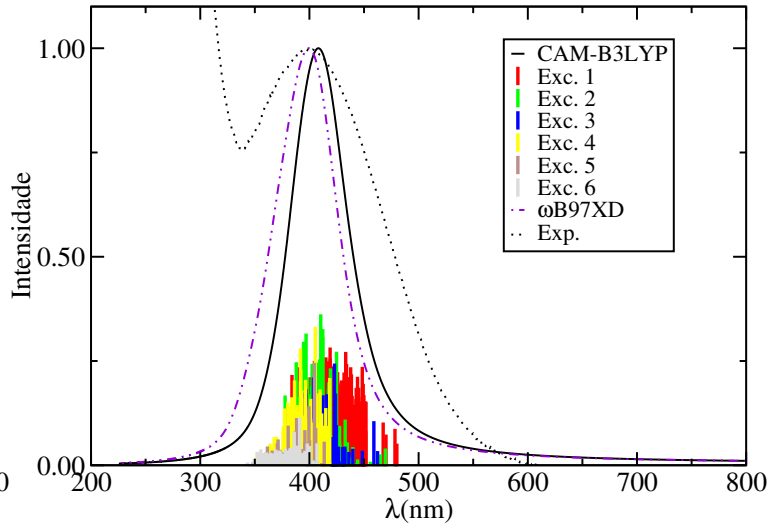

(b)

Figura 5.8: Espectros calculados a partir da convolução dos cálculos da energia de excitação (em acetonitrila, com o modelo PCM) dos complexos em configurações amostradas da dinâmica molecular de Born-Oppenheimer:(a) $C_{4}(4 C P)^{+} I^{-}$e (b) $C_{3} b i s(4 C P)^{2+} 2 I^{-}$.

correlação em lidar com as excitações de transferência de carga que, embora atenuadas pelas correções de longo alcance, não são sanadas totalmente. A dependência não é mais observada quando se inclui o solvente (no caso acetonitrila) usando o modelo contínuo PCM no cálculo das excitações, como pode ser visto na Figura 5.7b.

O modelo contínuo PCM foi então usado para incluir o efeito do solvente nas transições eletrônicas e assim obter o espectro em solução com base nas configurações da dinâmica de fase gasosa dos complexos. Os resultados são apresentados na Figura 5.8.

Para o $C_{4}(4 C P)^{+} I^{-}$, a inclusão do efeito do solvente resulta em um espectro com três picos estreitos e bem definidos, com máximos em 414, 303 e $265 \mathrm{~nm}$. Analisando os valores médios para cada excitação calculada, apresentados na Tabela 5.2, é possível notar que os picos são formados essencialmente pelas excitações 1, $4(\mathrm{HOMO} \rightarrow \mathrm{LUMO}+1)$ e $6(\mathrm{HOMO}-1 \rightarrow \mathrm{LUMO}+1$ e $\mathrm{HOMO}-2 \rightarrow \mathrm{LUMO}+1)$, cujos valores médios (calculados com CAM-B3LYP/PCM) são 415, 305 e $265 \mathrm{~nm}$, respectivamente. A banda de menor energia em $415 \mathrm{~nm}$ é formada essencialmente pela transição HOMO $\rightarrow$ LUMO. Para esse complexo não são observadas, portanto, mudanças significativas em relação aos resultados que já haviam sido obtidos com a geometria otimizada.

Quando se analisam os resultados para o $C_{3}$ bis $(4 C P)^{+2}+2 I^{-}$, no entanto, todas 
Tabela 5.2: Valores médios para o comprimento de onda ( $\lambda$ em nm) de excitação e respectivas força de oscilador $(f)$ para as 6 excitações eletrônicas de mais baixa energia.

\begin{tabular}{|c|c|c|c|c|c|c|}
\hline \multirow[b]{2}{*}{ Exc. } & \multicolumn{2}{|c|}{$\begin{array}{l}C_{4}(4 C P)^{+} I^{-} \\
\quad \text { isolado }\end{array}$} & \multicolumn{2}{|c|}{$\begin{array}{c}C_{4}(4 C P)^{+} I^{-} \\
\text {acetonitrila }(\mathrm{PCM})\end{array}$} & \multicolumn{2}{|c|}{$\begin{array}{l}C_{3} b i s(4 C P)^{+2}+2 I^{-} \\
\text {acetonitrila }(\mathrm{PCM})\end{array}$} \\
\hline & $\lambda$ & $f$ & $\lambda$ & $f$ & $\lambda$ & $f$ \\
\hline \multicolumn{7}{|c|}{ CAM-B3LYP } \\
\hline 1 & $529 \pm 77$ & 0.01 & $415 \pm 17$ & 0.25 & $417 \pm 15$ & 0.12 \\
\hline 2 & $517 \pm 77$ & 0.01 & $389 \pm 20$ & 0.01 & $405 \pm 14$ & 0.04 \\
\hline 3 & $461 \pm 49$ & 0.16 & $385 \pm 21$ & 0.02 & $400 \pm 14$ & 0.03 \\
\hline 4 & $359 \pm 27$ & 0.12 & $307 \pm 14$ & 0.14 & $388 \pm 12$ & 0.04 \\
\hline 5 & $326 \pm 27$ & 0.00 & $268 \pm 9$ & 0.01 & $378 \pm 11$ & 0.01 \\
\hline 6 & $311 \pm 26$ & 0.16 & $265 \pm 9$ & 0.10 & $373 \pm 11$ & 0.01 \\
\hline \multicolumn{7}{|c|}{$\omega \mathrm{B} 97 \mathrm{X}-\mathrm{D}$} \\
\hline 1 & $519 \pm 72$ & 0.02 & $410 \pm 15$ & 0.27 & $403 \pm 17$ & 0.16 \\
\hline 2 & $507 \pm 72$ & 0.01 & $381 \pm 18$ & 0.01 & $388 \pm 15$ & 0.02 \\
\hline 3 & $456 \pm 47$ & 0.16 & $377 \pm 19$ & 0.02 & $383 \pm 15$ & 0.02 \\
\hline 4 & $357 \pm 26$ & 0.12 & $305 \pm 14$ & 0.14 & $365 \pm 12$ & 0.04 \\
\hline 5 & $321 \pm 26$ & 0.00 & $265 \pm 8$ & 0.01 & $352 \pm 11$ & 0.01 \\
\hline 6 & $307 \pm 25$ & 0.15 & $262 \pm 8$ & 0.10 & $347 \pm 11$ & 0.01 \\
\hline
\end{tabular}


as excitações aparecem na mesma região, contribuindo para a formação de uma única banda, com máximo em 408 nm (CAM-B3LYP). A principal contribuição da banda vem da excitação $\mathrm{HOMO} \rightarrow$ LUMO, que em geral é primeira excitação e tem maior força de oscilador em média, como se observa na Tabela 5.2. Mas é importante ressaltar que há um ganho de intensidade nas demais excitações em relação as que foram calculadas para a estrutura de mínima energia, onde possuíam intensidade quase nula.

O resultado teórico para a banda de absorção do complexo $C_{3}$ bis $(4 C P)^{2+}+2 I^{-}$ exibe bom acordo para o máximo da banda experimental (400 nm). Conforme já foi observado estudando as estruturas de mínima energia, a estrutura IV é que mais contribui para a banda de absorção por ter maior força de oscilador. A exemplo do observado na estrutura de mínima energia, o segundo iodeto não afeta os resultados para a excitação eletrônica do complexo.

É importatne ressaltar que a dinâmica do par iônico foi realizada em vácuo (sem a inclusão do solvente) de forma que a interação entre os íons é mais forte do que seria em uma dinâmica em solução. Essa é uma das explicações para a banda teórica mais estreita do que a experimental. Além disso, os estudos com a geometria otimizada mostrados no capítulo anterior mostravam estruturas distintas para o $C_{3} b i s(4 C P)^{2+}$, mas durante os 50 ps de dinâmica foi observada apenas a estrutura IV usada como configuração inicial. O tempo de dinâmica provavelmente foi muito curto para que se pudesse observar as mudanças estruturais necessárias para amostrar as demais conformações.

Cada dinâmica de fase gasosa levou cerca de 60 dias em 512 processadores de um supercomputador. Assim, aumentar o tempo de simulação para tentar amostrar outras conformações ou mesmo realizar a dinâmica por primeiros princípios em solução, mesmo incluindo poucas moléculas de solvente, acaba sendo inviável do ponto de vista computacional. 


\subsection{Dinâmica molecular clássica}

A dinâmica molecular usando campos de força clássicos possui um custo computacional muito menor comparado à dinâmica por primeiros princípios, permitindo estudar os complexos com um número grande de moléculas de solvente, além de permitir dinâmicas com tempo de nanossegundos. A dinâmica molecular clássica (MD) foi realizada com o programa GROMACS (versão 4.5.4) [217-222, 258, 259].

\subsubsection{Parametrização e refinamento do campo de força}

A topologia do $C_{3} b i s(4 C P)^{2+}$ foi construída usando os parâmetros do campo de força OPLS para piridina [207,208], alcanos [197] e para íons halogênios [210] (ver Tabela 5.3). Alguns trabalhos na literatura usaram estes parâmetros para estudar a dinâmica de líquidos iônicos formados por derivados de piridínio semelhantes ao $C_{4}(4 C P)^{+}[44-50]$.

Incialmente foi realizada a dinâmica clássica do complexo $C_{3} b i s(4 C P)^{2+}+2 I^{-}$ isolado, usando condições semelhantes a dinâmica por primeiros princípios, como ensemble NVT e temperatura de $300 \mathrm{~K}$, passo de 0.25 fs e tempo total de simulação de 100 ps. A geometria de equilíbrio do $C_{3} b i s(4 C P)^{2+}+2 I^{-}$(estrutura IV) foi usada como ponto de partida nessa dinâmica. Assim foi possível avaliar a consistência da parametrização comparando as propriedades estruturais (comprimento de ligação, ângulos, etc.) obtidos da dinâmica clássica com as mesmas propriedades obtidas com a BOMD.

Duas importante modificações tiveram de ser realizadas a fim de que a dinâmica clássica fosse capaz de reproduzir os parâmetros estruturais da dinâmica por primeiros princípios: as cargas do potencial eletrostático e os parâmetros do iodeto.

As cargas para o potencial eletrostático foram inicialmente calculadas usando o método CHELPG [211], no nível de cálculo B3LYP e conjunto de funções bases 6$311+\mathrm{G}(\mathrm{d}, \mathrm{p})$ para H, C, N e bases e pseudopotencial aug-cc-pVDZ-PP para o iodo. Mas da mesma forma que ocorreu com o método de cargas naturais, quando os átomos de iodo são introduzidos explicitamente no cálculo, a carga em cada um deles é de apenas -0.63, ou seja, quase $40 \%$ da carga é transferida ao anel aromático. 
Tabela 5.3: Parâmetros ligados e não ligados do campo de força OPLS-AA usados na parametrização dos derivados de piridínio. NZ e CZ representam respectivamente o nitrogênio e carbono do grupo nitrila. NC, CA, e HA representam o nitrogênio, os carbonos e os hidrogênios do anel. CT e HC representam os carbonos e os hidrogênios da cadeia carbônica que une os anéis.

\begin{tabular}{|c|c|c|c|c|c|c|c|}
\hline \multirow[b]{2}{*}{ Grupo } & \multicolumn{3}{|c|}{ Parâmetros Não-ligados } & \multicolumn{4}{|c|}{ Parâmetros Ligados } \\
\hline & & $\begin{array}{c}\varepsilon \\
\left(\frac{k c a l}{m o l}\right)\end{array}$ & $\begin{array}{c}\sigma \\
(\AA)\end{array}$ & Ligações & $\begin{array}{c}K_{r} \\
\left(\frac{k c a l}{m o l . \AA^{2}}\right)\end{array}$ & Ângulos & $\begin{array}{c}K_{\theta} \\
\left(\frac{k c a l}{\text { mol.rad }^{2}}\right) \\
\end{array}$ \\
\hline \multirow[t]{2}{*}{ Nitrila [209] } & $\mathrm{NZ}$ & 0.170 & 3.20 & CZ-NZ & 650 & CA-CZ-NZ & 150 \\
\hline & $\mathrm{CZ}$ & 0.150 & 3.65 & $\mathrm{CA}-\mathrm{CZ}$ & 400 & CA-CA-CA & 63 \\
\hline \multirow[t]{3}{*}{ Piridina $[207,208]$} & $\mathrm{NC}$ & 0.170 & 3.25 & $\mathrm{CA}-\mathrm{CA}$ & 469 & CA-CA-HA & 35 \\
\hline & $\mathrm{CA}$ & 0.070 & 3.55 & CA-HA & 367 & CA-CA-NC & 70 \\
\hline & $\mathrm{HA}$ & 0.030 & 2.42 & $\mathrm{CA}-\mathrm{NC}$ & 483 & CT-CT-HC & 37.5 \\
\hline \multirow[t]{2}{*}{ Alcanos [197] } & $\mathrm{CT}$ & 0.066 & 3.50 & $\mathrm{HC}-\mathrm{CT}$ & 340 & CT-CT-CT & 58.35 \\
\hline & $\mathrm{HC}$ & 0.030 & 2.50 & $\mathrm{CT}-\mathrm{CT}$ & 268 & HC-CT-HC & 33 \\
\hline Iodeto [210] & $\mathrm{I}^{-}$ & 0.070 & 5.40 & & & & \\
\hline Iodo [210] & I & 0.600 & 3.750 & & & & \\
\hline
\end{tabular}

Por isso um novo conjunto de cargas, apresentado na Tabela 5.4, foi calculado fixando átomo de iodo como uma carga negativa (igual a -1) e calculando apenas as demais cargas do $C_{3} b i s(4 C P)^{2+}$ usando a metodologia CHELPG.

Para obter melhor concordância para o distanciamento contra-íon-anel também foram modificados os parâmetros de Lennard-Jones $(\epsilon$ e $\sigma)$ do $I^{-}$. Os parâmetros originais do OPLS faziam com que a distância do iodeto ao anel fosse em média $1 \AA$ maior na dinâmica clássica.

É importante ressaltar que existem na literatura diversos conjuntos distintos propostos para descrever os íons halogênios $\left(\mathrm{Cl}^{-}, \mathrm{Br}^{-}, \mathrm{I}^{-}\right.$, etc. ) [260]. Estes parâmetros variam consideravelmente entre si, embora sejam obtidos com diferentes campos de força. O valores de $\sigma$, por exemplo, varia de $3.5 \AA$ a $5.4 \AA$, sendo que este último corresponde justamente ao campo de força OPLS. Esses parâmetros são derivados para reproduzir a energia livre de hidratação dos íons. 
Tabela 5.4: Cargas parciais calculadas para o complexo usando a metodologia CHELPG. O cálculo foi realizado considerando o átomo de iodo como uma carga pontual. A carga dos demais átomos foram calculadas usando o funcional B3LYP e a base $6-311+G(d, p)$.

\begin{tabular}{lrr}
\hline \hline & Vácuo & Acetonitrila $(\mathrm{PCM})$ \\
\hline $\mathrm{N} 1$ & -0.326 & -0.403 \\
$\mathrm{C} 2$ & 0.245 & 0.333 \\
$\mathrm{C} 3$ & 0.294 & 0.212 \\
$\mathrm{C} 4$ & -0.245 & -0.167 \\
$\mathrm{C} 5$ & -0.268 & -0.167 \\
$\mathrm{C} 6$ & 0.152 & 0.124 \\
$\mathrm{C} 7$ & 0.240 & 0.136 \\
$\mathrm{~N} 8$ & -0.130 & -0.015 \\
$\mathrm{C} 9$ & -0.106 & -0.093 \\
$\mathrm{C} 10$ & 0.301 & 0.186 \\
$\mathrm{C} 11$ & -0.213 & -0.159 \\
$\mathrm{~N} 12$ & 0.109 & 0.162 \\
$\mathrm{C} 13$ & 0.017 & 0.015 \\
$\mathrm{C} 14$ & 0.010 & 0.008 \\
$\mathrm{C} 15$ & -0.124 & -0.086 \\
$\mathrm{C} 16$ & -0.111 & -0.074 \\
$\mathrm{C} 17$ & 0.129 & 0.108 \\
$\mathrm{C} 18$ & 0.293 & 0.357 \\
$\mathrm{~N} 19$ & -0.338 & -0.407 \\
$\mathrm{H} 20$ & 0.167 & 0.169 \\
$\mathrm{H} 21$ & 0.230 & 0.196 \\
$\mathrm{H} 22$ & 0.184 & 0.173 \\
$\mathrm{H} 23$ & 0.156 & 0.155 \\
$\mathrm{H} 24$ & 0.062 & 0.111 \\
$\mathrm{H} 25$ & 0.190 & 0.134 \\
$\mathrm{H} 26$ & 0.025 & 0.027 \\
$\mathrm{H} 27$ & -0.025 & 0.034 \\
$\mathrm{H} 28$ & 0.168 & 0.115 \\
$\mathrm{H} 29$ & 0.127 & 0.101 \\
$\mathrm{H} 30$ & 0.236 & 0.195 \\
$\mathrm{H} 31$ & 0.245 & 0.193 \\
$\mathrm{H} 32$ & 0.153 & 0.164 \\
$\mathrm{H} 33$ & 0.153 & 0.163 \\
$\mathrm{I} 35$ & -1.000 & -1.000 \\
\hline \hline & -1.000 & \\
\hline
\end{tabular}



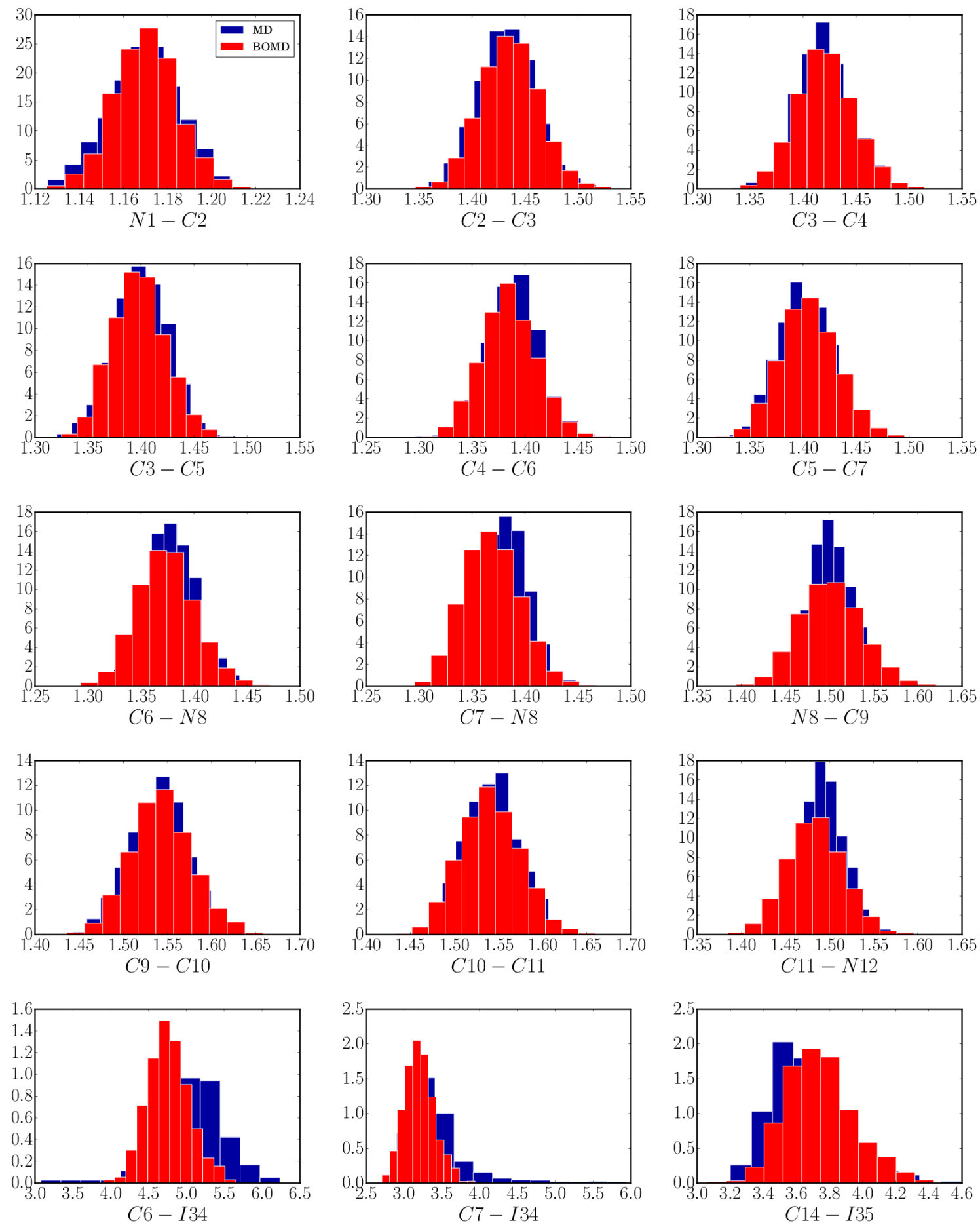

Figura 5.9: Comparação entre os comprimentos de ligação e entre algumas distâncias $C---I$ obtidos coma dinâmica clássica (MD) e com a dinâmica molecular de Born-Oppenheimer (BOMD) 

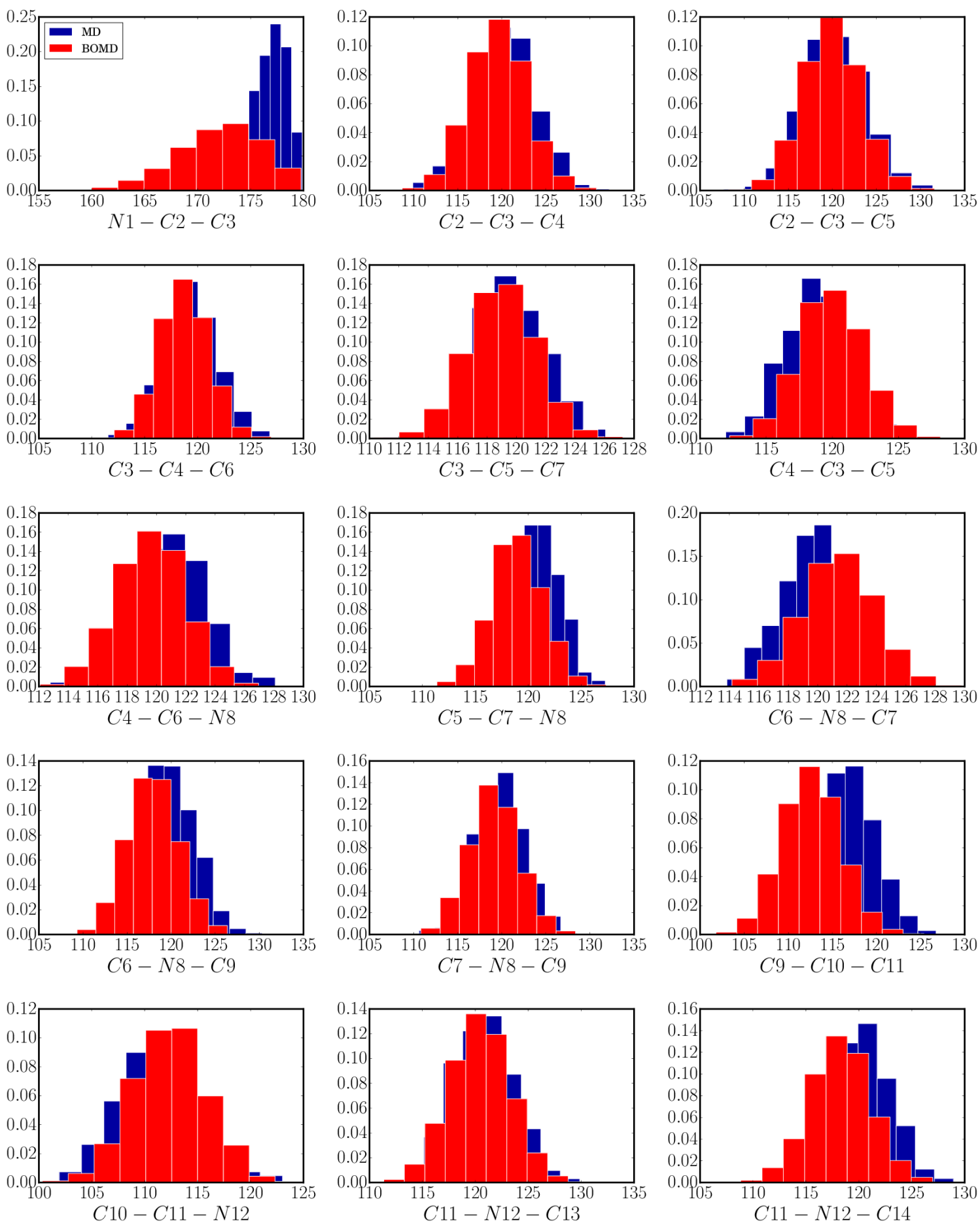

Figura 5.10: Comparação entre os ângulos de ligação obtidos com a dinâmica clássica (MD) e com a dinâmica molecular de Born-Oppenheimer (BOMD) 

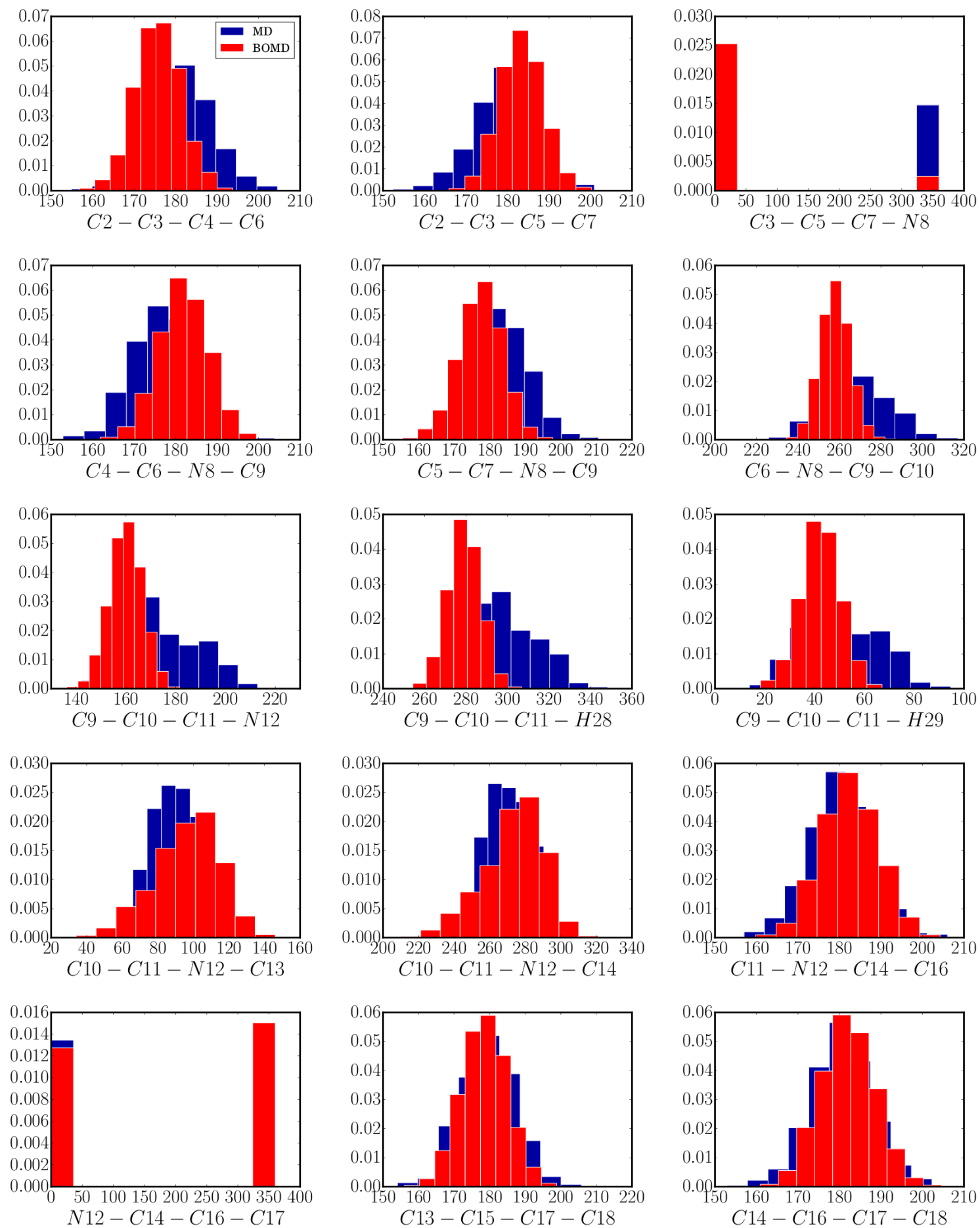

Figura 5.11: Comparação entre os ângulos diedros obtidos com a dinâmica clássica (MD) e com a dinâmica molecular de Born-Oppenheimer (BOMD) 
Em vez de escolher arbitrariamente parâmetros para o $I^{-}$em outro campo de força, nossa proposta foi usar os parâmetros $\epsilon=0.60 \mathrm{kcal} / \mathrm{mol}$ e $\sigma=3.75 \AA$, que no campo de força OPLS são usados para descrever o átomo de iodo ligado a um anel aromático [210]. Como o nosso objetivo é a descrição do iodeto no complexo, esses parâmetros se mostram mais adequados uma vez que naturalmente permitem tratar tanto a aproximação quanto a interação com o anel.

Essas modificações foram suficientes para que o campo de força clássico conseguisse reproduzir muito bem as distâncias, ângulos e diedros obtidos na dinâmica por primeiros princípios, conforme mostram os histogramas mostrados na Figura 5.9, na Figura 5.10 e na Figura 5.11.

\subsubsection{Dinâmica do $C_{3} b i s(4 C P)^{2+} \operatorname{com} 2 I^{-}$em acetonitrila}

Uma vez constatado que o campo de força clássico era capaz de produzir resultados em acordo com os da BOMD, o próximo passo foi a realização da dinâmica do complexo formado pelo $C_{3}$ bis $(4 C P)^{2+}$ com $2 I^{-}$em acetonitrila. As cargas usadas no campo de força foram calculadas descrevendo os iodetos como carga pontuais e usando o modelo contínuo PCM para descrever o solvente (ver Tabela 5.4). O arquivo de topologia usado nesta etapa está disponível no Apêndice D.

A dinâmica foi realizada em uma caixa cúbica de largura igual a $50 \AA$, no emsemple NpT, à pressão de 1 atm e temperatura de 300K. Usamos o barostato de Berendsen e o termostato estocástico de escalonamento de velocidades. O número de total de moléculas de acetonitrila foi igual a 1284. O tempo de integração de 0.2 fs. Para a acetonitrila, o campo de força foi o modelo OPLS-AA de 6 sítios (maiores detalhes no Apêndice C). Usamos um raio de corte de 14 A e PME para a correção de longo alcance do termo de Coulomb. O tempo total da dinâmica foi igual a 24 ns. O arquivo de entrada completo também pode ser encontrado no Apêndice D.

A Figura 5.12 reúne a evolução temporal de diversas propriedades estruturais que são úteis para a caracterização das conformações do complexo. São estas as distâncias C7-I34, C6-I35, C13-I34 e C14-I35, mostradas na Figura 5.12b. Os diedros N8-C9C10-C11 e C9-C10-C11-N12, mostrados na Figura 5.12c. Os diedros C7-N8-C9-C10 e C10-C11-N12-C13, mostrados na figura 5.12e. 


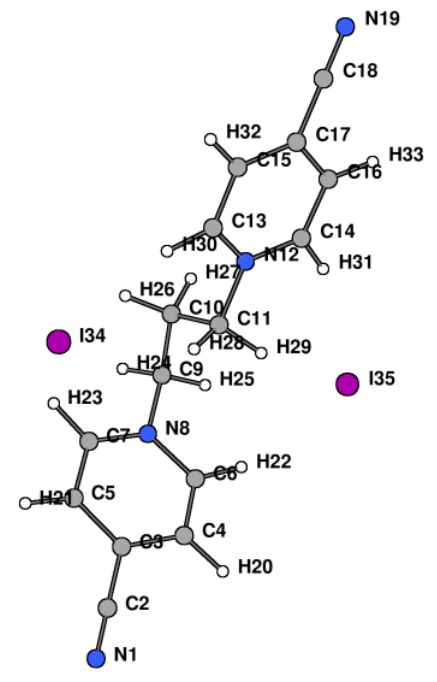

(a)

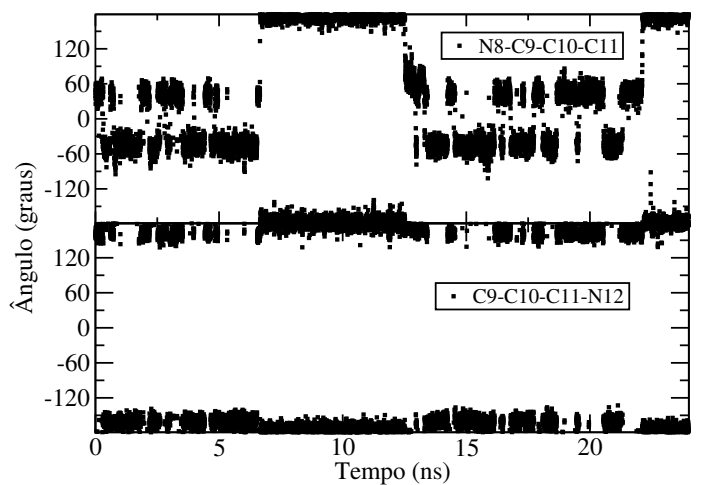

(c)

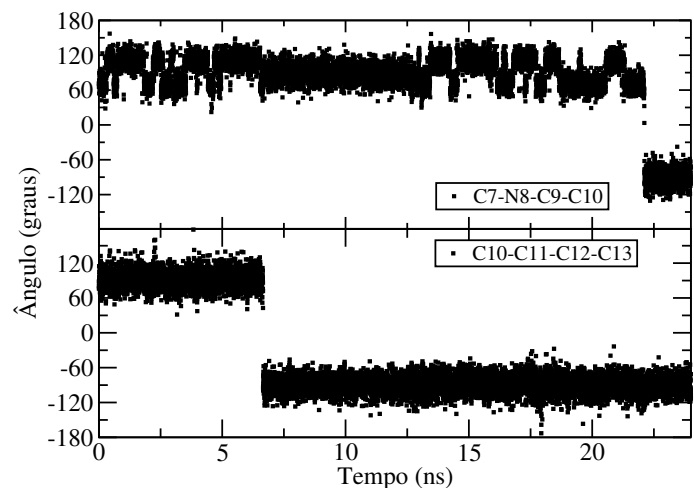

(e)

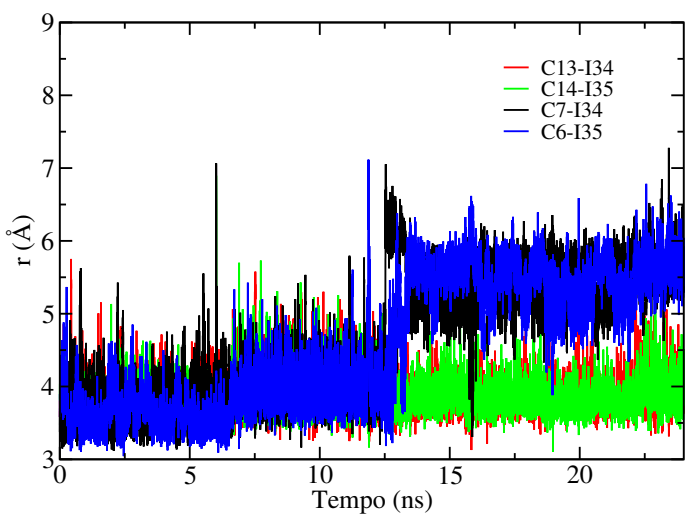

(b)

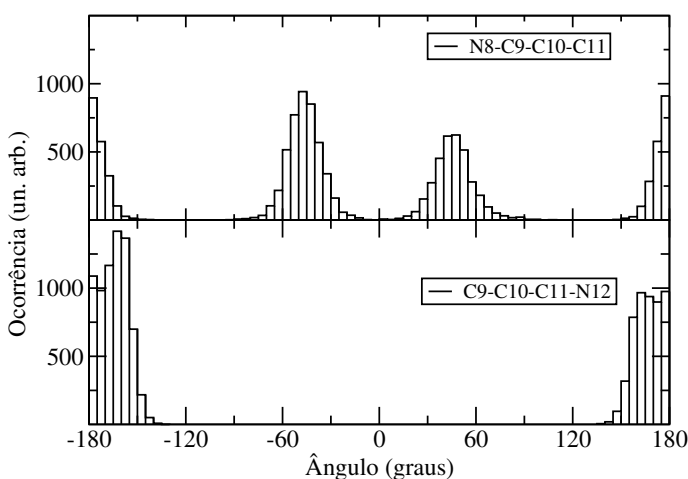

(d)

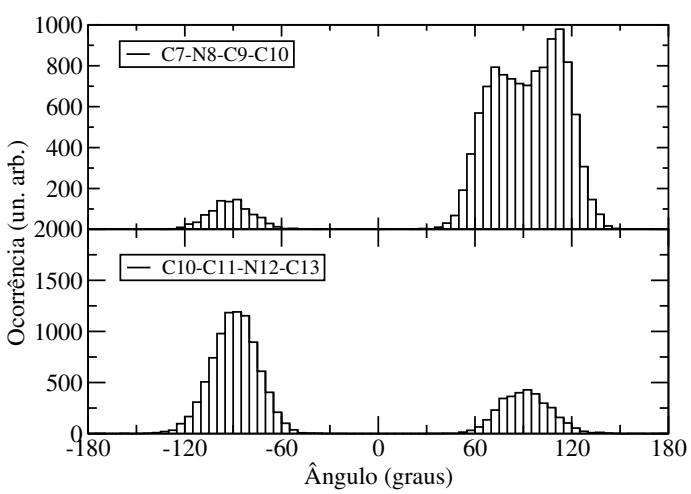

(f)

Figura 5.12: Evolução temporal de alguns parâmetros estruturais ao longo de dinâmica molecular clássica. (a) Numeração atômica adotada para o complexo (b) distâncias CI. (c) diedros N8-C9-C10-C11 e C9-C10-C11-N12 (d) histograma com a distribuição dos diedros N8-C9C10-C11 e C9-C10-C11-N12 (e) diedros C7-N8-C9-C10 e C10-C11-N12-N13 (f) histograma com a distribuição dos diedros C7-N8-C9-C10 e C10-C11-N12-N13. 
Ao longo da dinâmica molecular não houve dissociação do complexo, de modo que os iodetos permaneceram próximos aos anéis aromáticos durante todo o tempo. Também não foi observada a conformação sanduíche (semelhante a estrutura II), que seria a mais próxima da proposta experimental.

Foram amostradas duas conformações para o complexo, semelhantes as conformações IV e V obtidas com otimização de geometria e estudadas no capítulo anterior.

Note que ao longo da dinâmica o ângulo diedral C9-C10-C11-N12 não muda consideravelmente, flutuando ao redor de $180^{\circ}$ (em módulo). As mudanças conformacionais ficam caracterizadas sobretudo por mudanças no diedro N8-C9-C10-C11.

No intervalo entre 0 ps e 6.6 ns (ou 6600 ps), a conformação do complexo é semelhante a estrutura inicial do complexo (estrutura IV). Neste intervalo o diedro N8-C9-C10-C11 flutua ao redor de $\pm 50^{\circ}$. O valor positivo corresponde a estrutura inicial do complexo. Já o valor negativo corresponde a uma forma espelhada desta estrutura (considerando um plano perpendicular ao anel formado pelos átomos N12C13-C14-C15-C16). Veja, por exemplo, as estruturas em $t=1.0 \mathrm{~ns}(t=1000 \mathrm{ps}$ ) e $t=6.0 \mathrm{~ns}(t=6000 \mathrm{ps})$ na Figura 5.13. Por causa da simetria das estruturas, ambas devem apresentar propriedades semelhantes, em particular no que se refere ao espectro de absorção.

A primeira mudança conformacional significativa acontece em $t=6.65$ ns e é caracterizada por mudanças nos diedros N8-C9-C10-C11 e C10-C11-N12-C13, cujos valores passam a flutuar ao redor de $\pm 180^{\circ}$ no primeiro caso e ao redor de -90 no segundo caso. A conformação é semelhante a estrutura $\mathbf{V}$.

O complexo volta a ter uma conformação semelhante a estrutura IV em 12.5 ns. Observa-se um aumento da distância C7-I34 e C6-I35, ocasionada pela mudança no diedro N8-C9-C10-C11 para valores próximos a $\pm 50^{\circ}$, enquanto o diedro C10-C11N12-N13 continua ao redor $-90^{\circ}$.

Finalmente em 22.1 ns o complexo volta a estrutura V com o diedro N8-C9-C10$\mathrm{C} 11$ próximo a $\pm 180^{\circ}$. Durante a mudança ocorre um giro de $180^{\circ}$ ao redor do eixo no anel $\mathrm{C} 4-\mathrm{C} 5-\mathrm{C} 6-\mathrm{C} 7-\mathrm{N} 8$, de forma que o ângulo $\mathrm{C} 7-\mathrm{N} 8-\mathrm{C} 9-\mathrm{C} 10$ muda para valores próximos a $-90^{\circ}$ e não há variação nas distâncias $\mathrm{C}-\mathrm{I}$ em relação à estrutura anterior (Note inversão dos átomos C6 e C7 em $t=12520$ ps e $t=22124$ ps na Figura 5.13). 


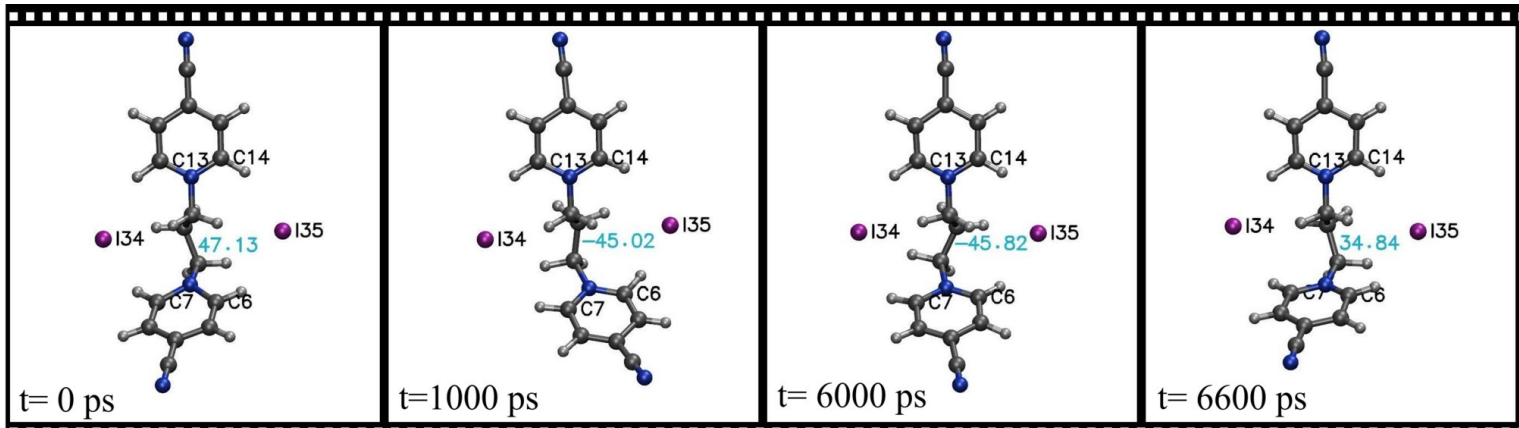

m

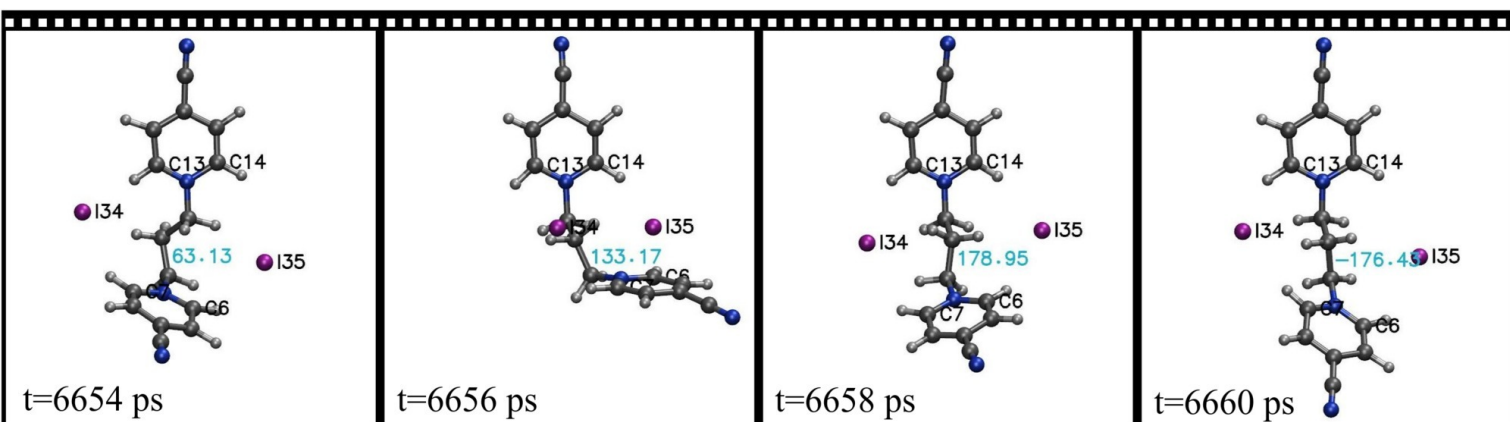

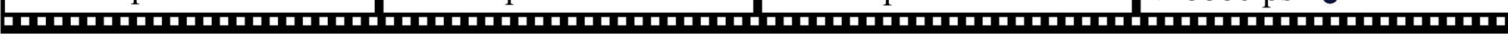
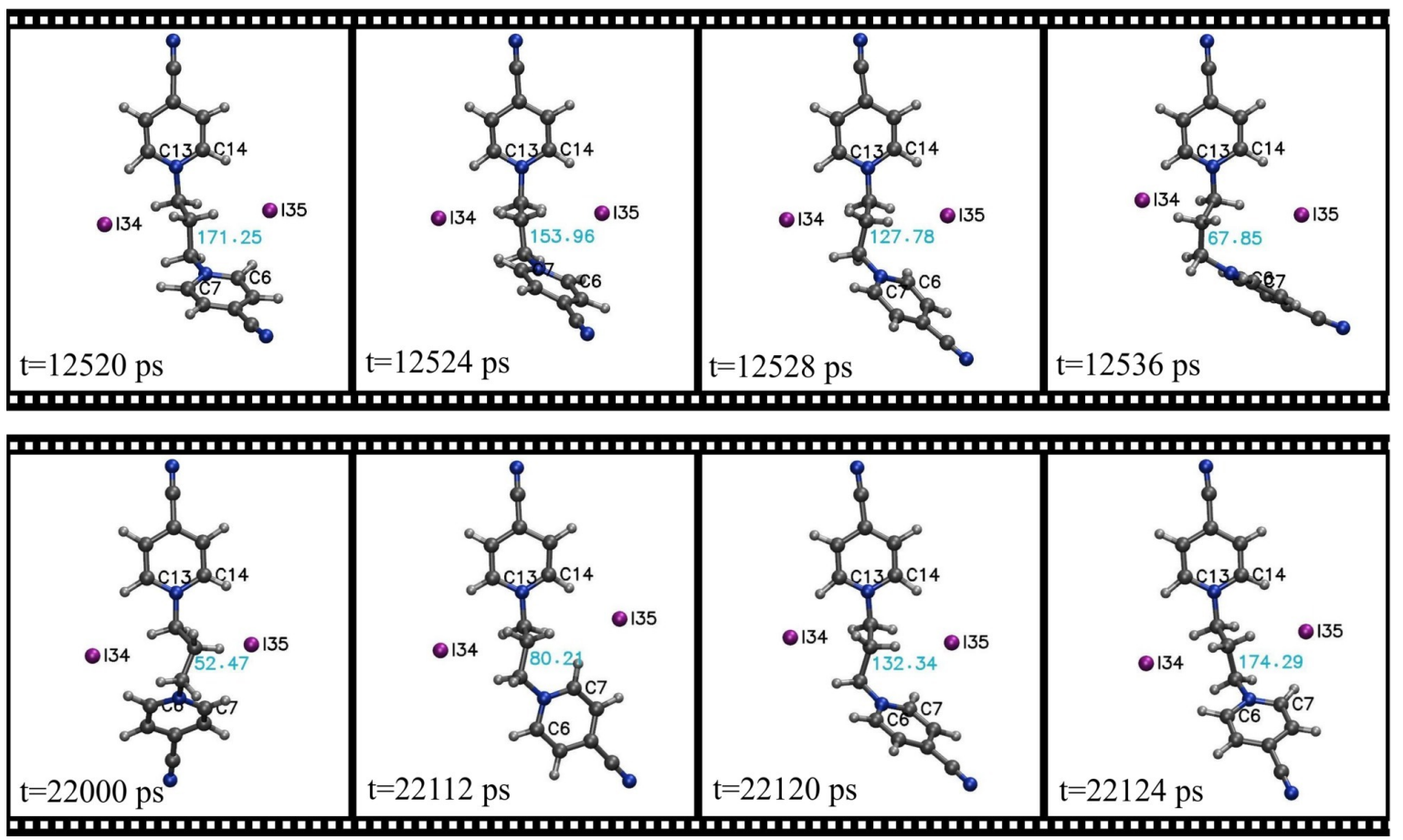

Figura 5.13: Representação da evolução estrutural do complexo ao longo da dinâmica, com ênfase nos intervalos onde são observadas mudanças conformacionais. O valor do diedro N8-C9-C10-C11 também é apresentado. 


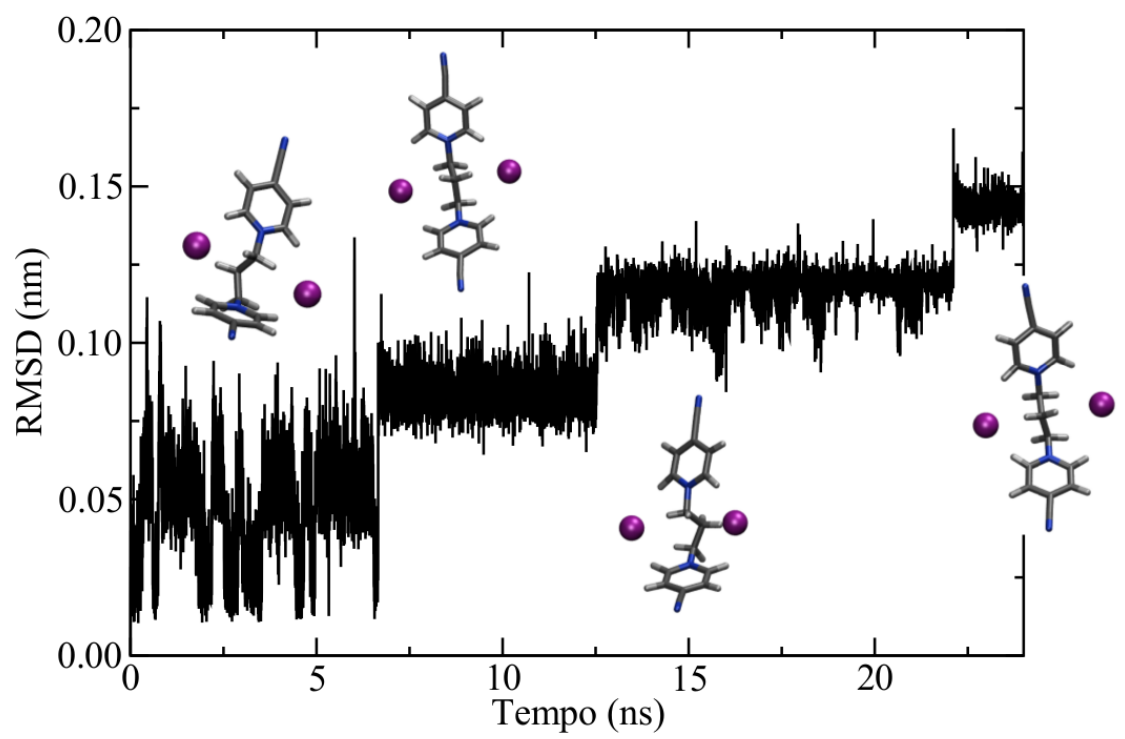

Figura 5.14: Evolução temporal do RMSD das estruturas do complexo em acetonitrila

As estruturas também ficam bem caracterizadas quando se oberva a evolução temporal do RMSD em relação à estrutura inicial, mostrada na Figura 5.14. As mudanças conformacionais ficam evidentes em $6.6 \mathrm{~ns}, 12.5 \mathrm{~ns}$ e $22.1 \mathrm{~ns}$, caracterizadas por saltos no valor do RMSD.

O RMSD pode ser usado também para caracterizar as estruturas amostradas em solução, agrupando cada uma delas em grupos ou "clusters" de acordo com o seu grau de semelhança (ver seção 3.8.3). Usando um raio de corte para o RMSD de $0.05 \mathrm{~nm}$ e o algoritmo gromos [229] implementado na ferramenta "g_cluster" do programa GROMACS, foram encontrados seis conjuntos de estruturas. As estruturas mais representativa de cada grupo, são apresentadas Figura 5.15.

As estruturas da Figura 5.15a e da Figura 5.15e são semelhantes a estrutura V, amostradas entre 6.6-12.5 ns e entre 22.1-24 ns, respectivamente. Conforme já mencionado anteriormente a diferença entre elas está na rotação em torno do eixo no anel N12-C13-C14-C15-C16-C17. Esta rotação tem impacto sobre o RMSD mas, por causa da simetria do anel, não altera nenhuma outra propriedade de interesse. As demais estruturas são semelhantes a estrutura IV. A Figura 5.15b e a Figura 5.15c correspondem a estruturas simétricas amostradas no intervalo entre 12.5 ns a 22.1 ns. 


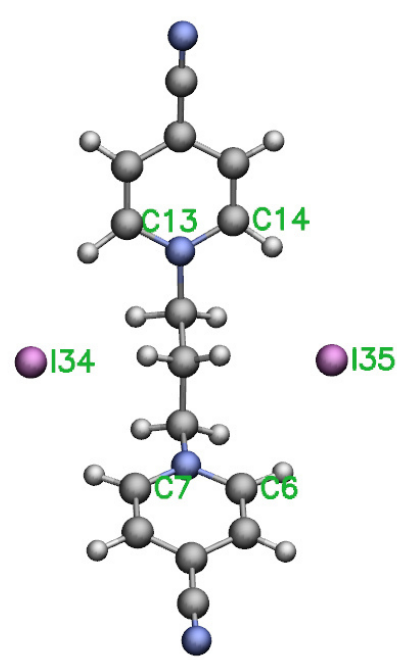

(a) $26 \%$

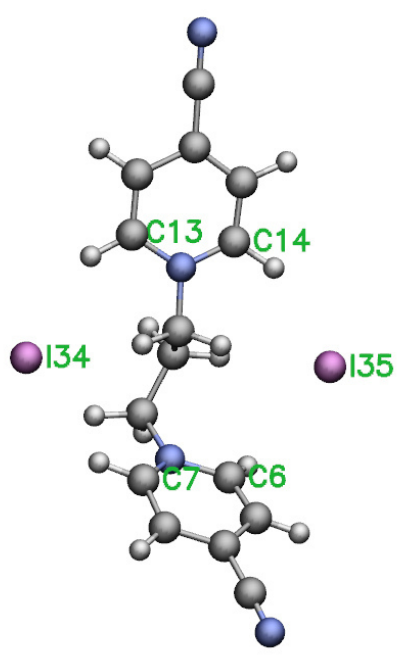

(d) $10 \%$

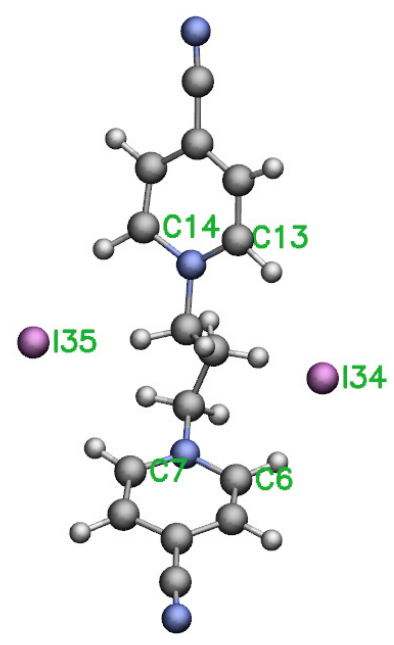

(b) $22 \%$

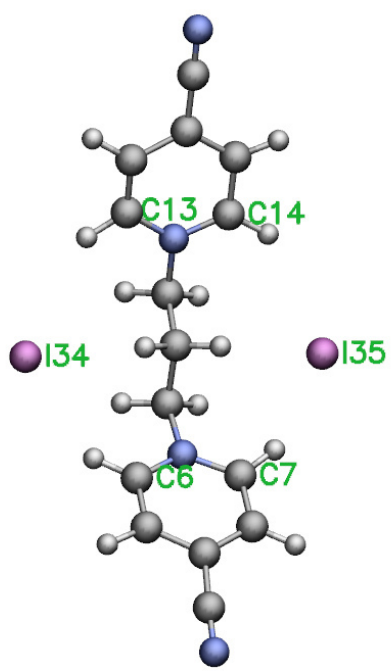

(e) $9 \%$

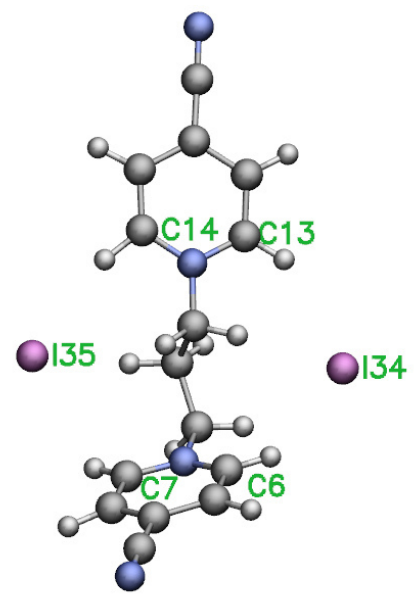

(c) $20 \%$

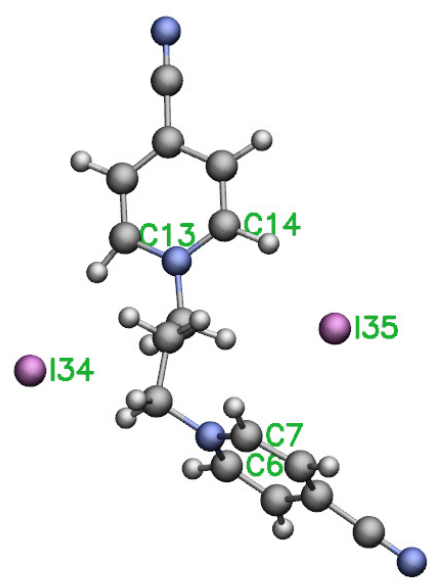

(f) $3 \%$

Figura 5.15: Clusters moleculares obtidos da dinâmica clássica em acetonitrila: $C_{3} \operatorname{bis}(4 C P)^{2+}$

Finalmente, a Figura 5.15e representa estruturas no intervalo entre 4.0 ns e 6.6 ns e a Figura $5.15 \mathrm{f}$ entre 12.5 ns e 22.1 ns. Assim, os seis grupos encontrados pelo algoritmo de clustering se reduzem aos dois tipos de estrutura já estudados anteriormente. 


\subsubsection{Cálculo do espectro de absorção em solução}

Para o cálculo do espectro de absorção em solução foram selecionadas 200 configurações amostradas uniformemente a cada 100 ps. Em cada configuração foram calculadas as 6 energias de excitação de mais baixa energia usando o funcional CAM-B3LYP. Cada configuração foi constituída pelo complexo e pelas moléculas de acetonitrila mais próximas, representadas como cargas pontuais.

O espectro de absorção calculado, mostrado na Figura 5.16, mostra novamente uma excelente concordância com a medida experimental e o máximo da banda calculada coincide com o máximo da banda experimental, em $400 \mathrm{~nm}$.

A fim de avaliar a contribuição de cada conformação para o espectro de absorção do CTC, foram calculados espectros separando conformações dois grupos, formados 122 e 78 estruturas semelhantes as estruturas IV e V, respectivamente. Os espectros são apresentados na Figura 5.17. As duas conformações têm bandas de absorção muito semelhantes, mas as conformações que mais contribuem para o espectro são aquelas

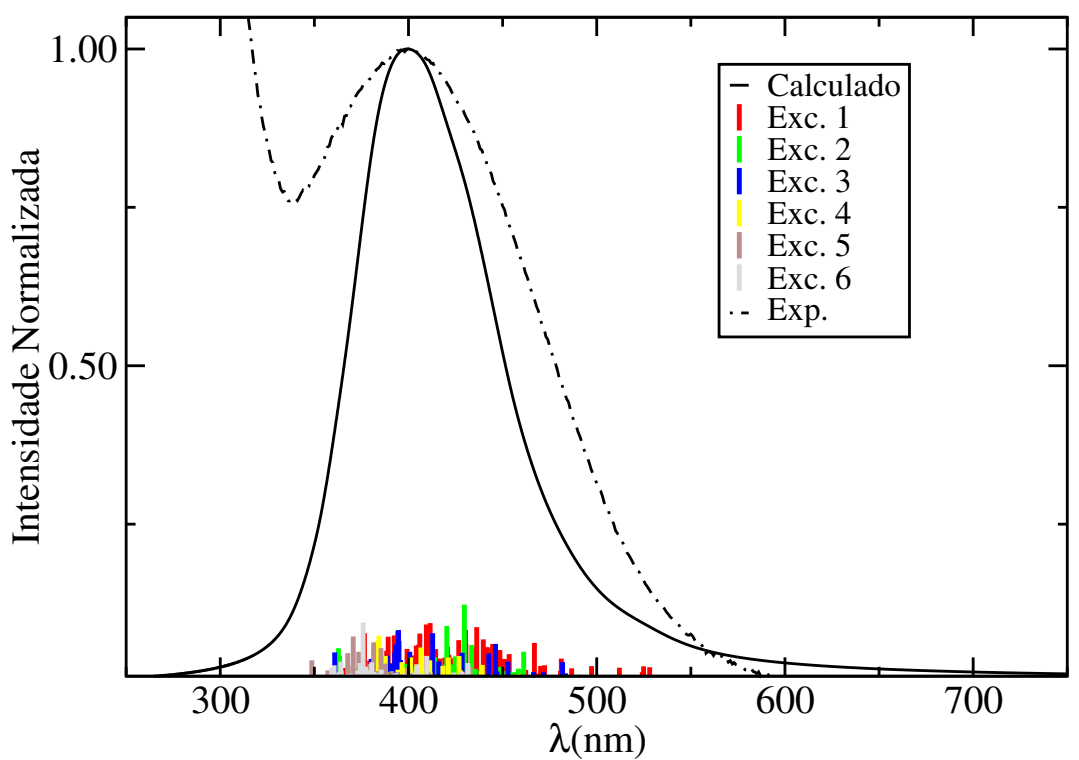

Figura 5.16: Espectro eletrônico de absorção calculado para o complexo $C_{3} b i s(4 C P)^{2+}+2 I^{-}$ em acetonitrila (solvente representado por 750 moléculas incluídas no calculo com cargas pontuais). O espectro foi calculado ajustando-se em energia lorentzianas de largura $0.1 \mathrm{eV}$ nas 6 excitações de mais baixa energia, calculadas usando o funcional CAM-B3LYP, em 200 configurações amostradas da dinâmica clássica. As intensidades das excitações verticais correspondem as forças de oscilador. 


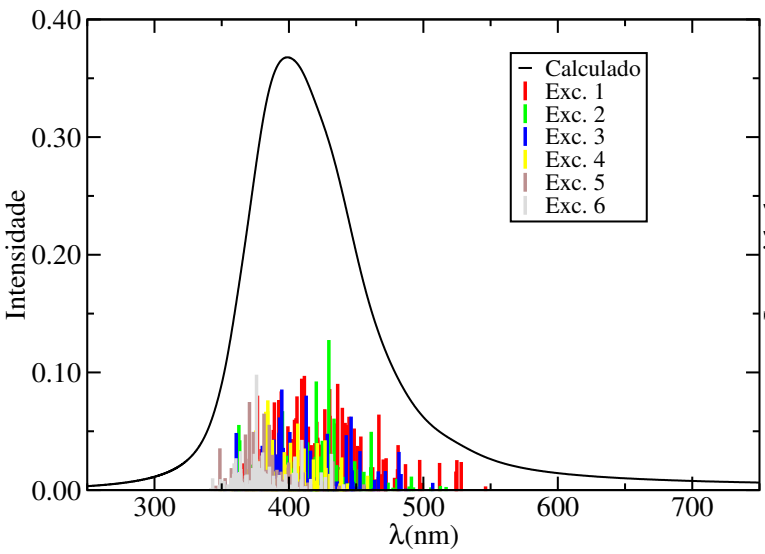

(a)

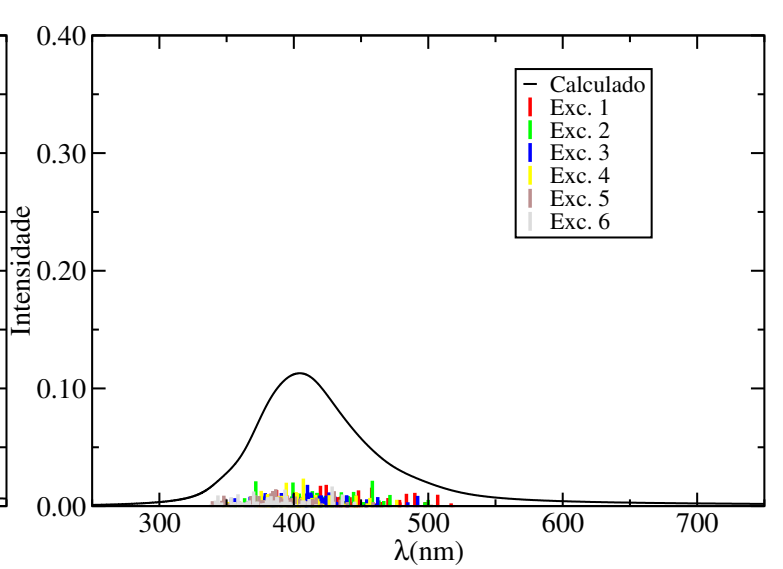

(b)

Figura 5.17: Espectro calculado para o $C_{3} b i s(4 C P)^{2+}+2 I^{-}$separando as estruturas por similaridade. (a) Espectro calculado usando 122 estruturas semelhantes a estrutura IV. (b) Espectro calculado usando 78 estruturas semelhantes a estrutura V.

que se assemelham a estrutura IV. A contribuição das estruturas do tipo $\mathbf{V}$ é muito menor, já que possuem intensidades quase nulas.

Resta ainda avaliar o efeito da inclusão de moléculas explícitas de solvente no cálculo das energias de excitação do complexo. Todos os resultados apresentados até aqui sugerem que apenas a inclusão da interação eletrostática soluto-solvente já é suficiente para descrever o espectro de absorção em acetonitrila.

Na Figura 5.18 são apresentados alguns gráficos da distribuição radial de moléculas de solvente ao redor do complexo em solução. A análise da função de distribuição de mínima distância entre o complexo e o solvente - Figura 5.18a - mostra que a primeira camada de solvatação se estende de $1.9 \AA$ até $4.3 \AA$. A integração desse trecho da curva mostra que essa camada é composta por 30 moléculas de acetonitrila. A segunda camada de solvatação se estende de $4.3 \AA$ até $8.4 \AA$ e é composta por 76 moléculas de acetonitrila.

Uma análise semelhante é obtida quando se analisa a RDF entre os íons I34, e I35 e o carbono C1 do grupo metil da acetonitrila - Figura 5.18b. Os dois $I^{-}$apresentam distribuições idênticas em relação ao solvente. 6 moléculas de acetonitrila formam a primeira camada de solvatação ao redor dos átomos de iodo, e esta camada se estende de $3.0 \AA$ até $5.2 \AA$. 


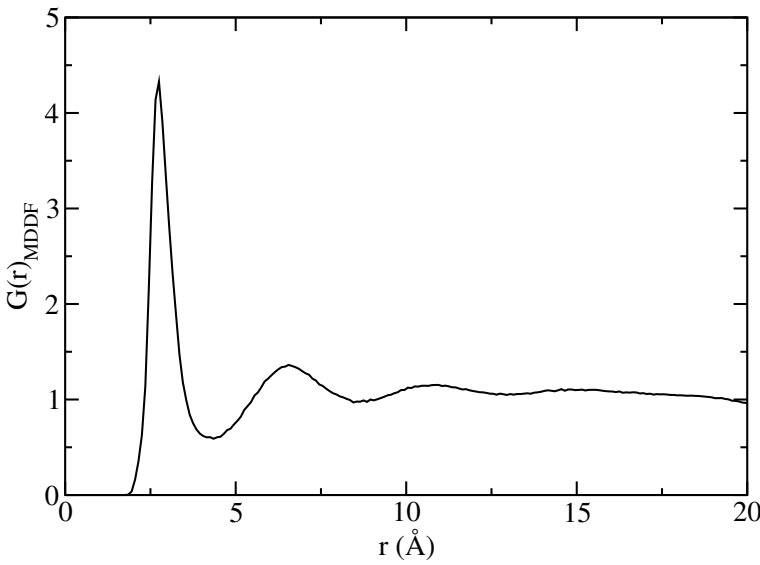

(a)

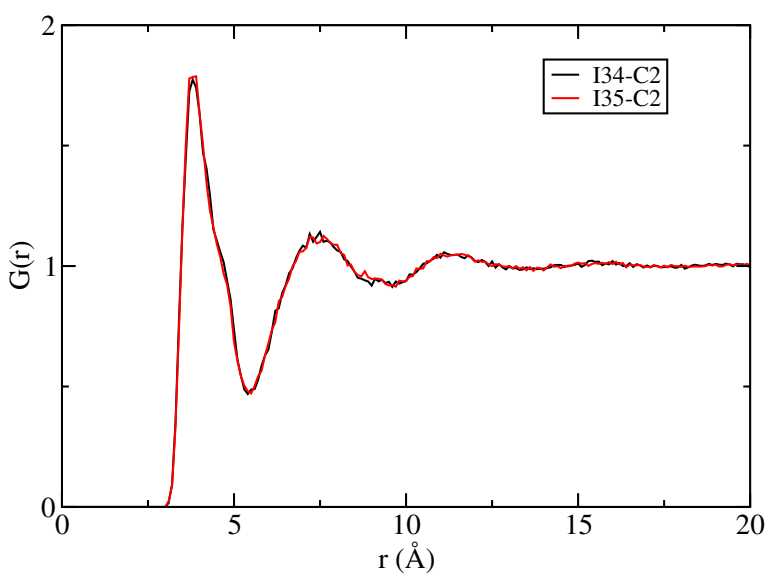

(c)

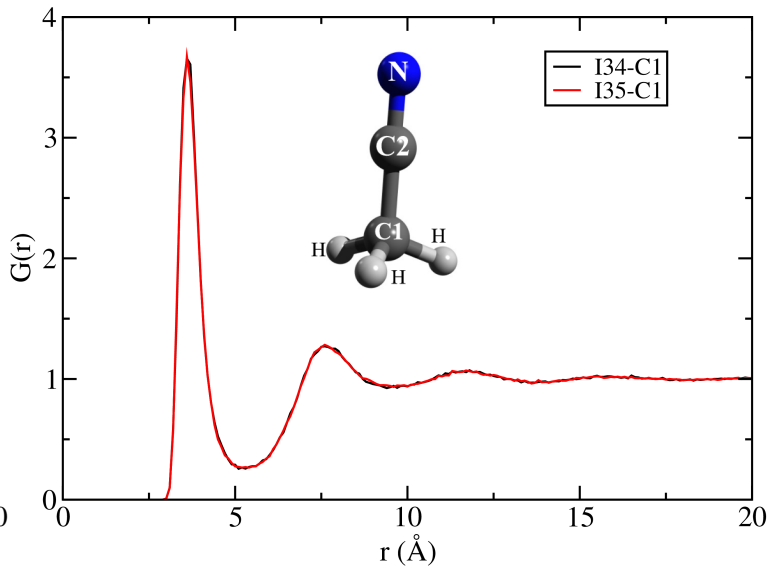

(b)

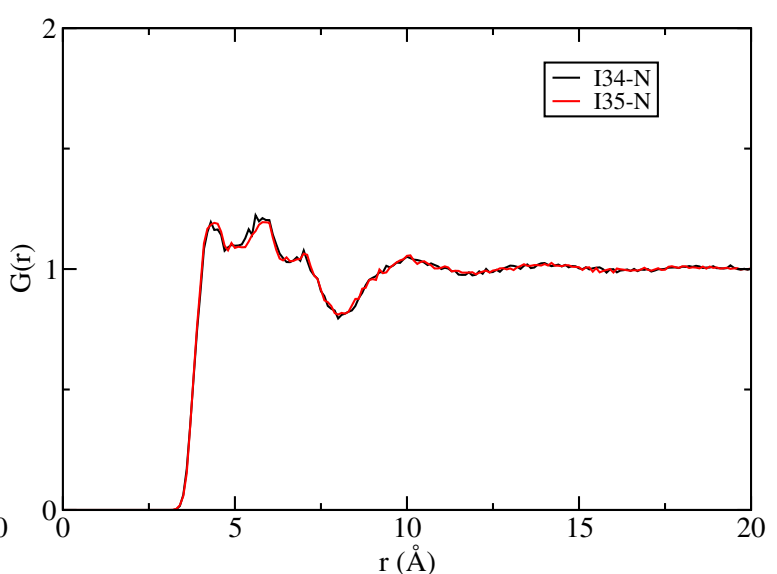

(d)

Figura 5.18: Função de distribuição radial pares entre o $C_{3} b i s(4 C P)^{2+}$ e acetonitrila.

Embora os resultados do solvente com carga pontual já estejam em excelente concordância com o resultado experimental, a influência da inclusão de moléculas explícitas de acetonitrila sobre as energias de excitação do sistema foi investigada. Devido o alto custo computacional, apenas uma configuração foi selecionada. Na tabela 5.5 são apresentados os valores de energia de excitação para essa configuração, comparando o valor das 6 excitações com a adição 6 e 30 moléculas explícitas de solvente e com o restante sendo incluindo como cargas pontuais.

Os resultados da tabela corroboram com o que havia sido observado anteriormente, ou seja, que interação eletrostática soluto-solvente é principal contribuição 
Tabela 5.5: Comprimento de onda de excitação (em nm) calculadas em uma dada configuração com a inclusão de moléculas explícitas de acetonitrila, e restante como cargas pontuais. Força de oscilador entre parênteses.

\begin{tabular}{lccc}
\hline \hline No Acetonitrilas com carga pontual & 750 & 744 & 720 \\
No Acetonitrilas explícitas & 0 & 6 & 30 \\
\hline Exc. 1 & $412(0.10)$ & $413(0.09)$ & $412(0.09)$ \\
Exc. 2 & $391(0.00)$ & $405(0.00)$ & $402(0.00)$ \\
Exc. 3 & $386(0.00)$ & $393(0.01)$ & $391(0.01)$ \\
Exc. 4 & $368(0.01)$ & $362(0.01)$ & $370(0.01)$ \\
Exc. 5 & $361(0.01)$ & $353(0.00)$ & $360(0.00)$ \\
Exc. 6 & $349(0.01)$ & $351(0.01)$ & $358(0.01)$ \\
\hline \hline
\end{tabular}

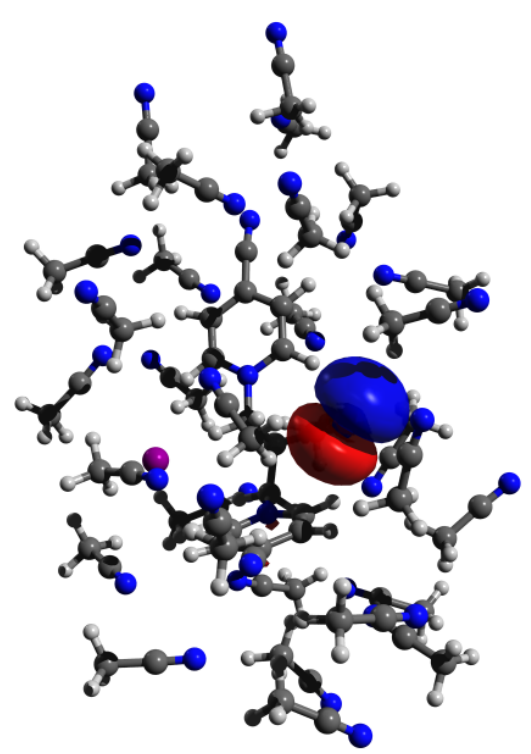

(a)

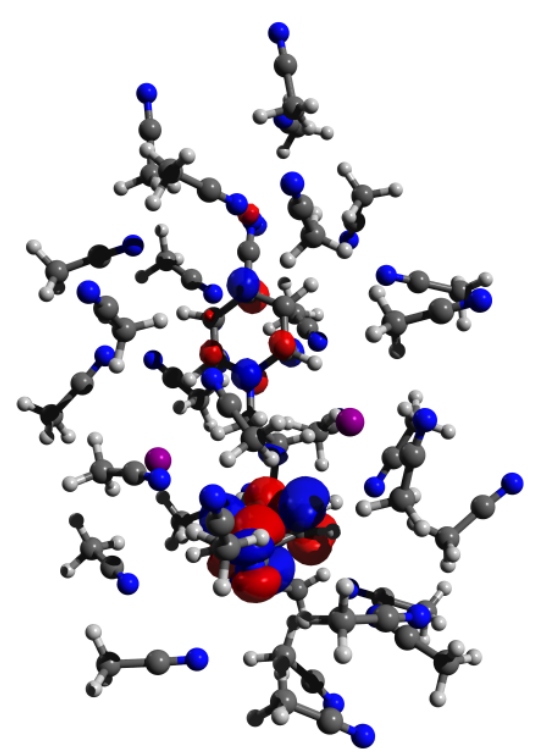

(b)

Figura 5.19: Orbitais moleculares calculados para o complexo $C_{3} b i s(4 C P)^{2+}$ e juntamente com a primeira camada de solvatação formada por 30 moléculas de acetonitrila:(a) HOMO e (b) LUMO 
para o efeito do meio sobre a banda de transferência de carga. Na Figura 5.18 estão representados os orbitais HOMO e LUMO para a configuração composta pelo complexo e 30 moléculas de acetonitrila. Note que os orbitais moleculares são localizados apenas sobre o complexo, não havendo delocalização sobre moléculas do solvente.

A dinâmica clássica do complexo deixou claro aquilo que já apontavam os resultados de vácuo, ou seja, a existência de 2 grupos de estruturas para o complexo em solução. O complexo alterna entre as estruturas IV e $\mathbf{V}$ durante a dinâmica molecular e a estrutura IV é a que mais contribui para o espectro.

Com esse campo de força em mãos, agora a dinâmica clássica pode ser agora facilmente empregada no estudo de outros sistemas semelhantes ou mesmo outro solventes. Algumas dessas aplicações serão exploradas na próxima seção.

\subsection{Extensão dos resultados do campo de força}

Um estudo abrangente da aplicação da dinâmica molecular clássica para outros sistemas foge ao escopo deste trabalho. Ainda sim, a validade do campo de força foi testada em duas situações adicionais: na dinâmica do $C_{3} b i s(4 C P)^{2+}$ em água e na dinâmica do $C_{8}$ bis $(4 C P)^{2+}$ em acetonitrila.

\subsubsection{Complexo formado pelo $C_{3} b i s(4 C P)^{2+} \operatorname{com} 2 \mathbf{I}^{-}$em água}

A dinâmica do complexo $C_{3}$ bis $(4 C P)^{2+}+2 I^{-}$em solução aquosa foi realizada usando o modelo SPC para a água e os demais critérios já empregados na dinâmica do complexo em acetonitrila: ensemble NpT, temperatura de $300 \mathrm{~K}$, pressão de $1 \mathrm{~atm}$, passo de 0.002 fs, tempo total de $24 \mathrm{~ns}$, etc.

A Figura 5.20 apresenta a evolução temporal das distâncias C6-I34, C7-I35, C13-I34, C14-I35 e dos diedros N8-C9-C10-C11 e C9-C10-C11-N12, bem como a representação de um estado do sistema onde se observa a total dissociação dos íons, ao contrário do ocorria em acetonitrila. Mesmo quando os contra-íons se aproximam do $C_{3}$ bis $(4 C P)^{2+}$, com entre 5 e 10 ns e após 20 ns de dinâmica, a distância é maior do que a observada em acetonitrila. Além disso, como se observa na Figura 5.20b os diedros N8-C9-C10-C11 e C9-C10-C11-N12 permanecem próximos a $\pm 180^{\circ}$, de forma que 


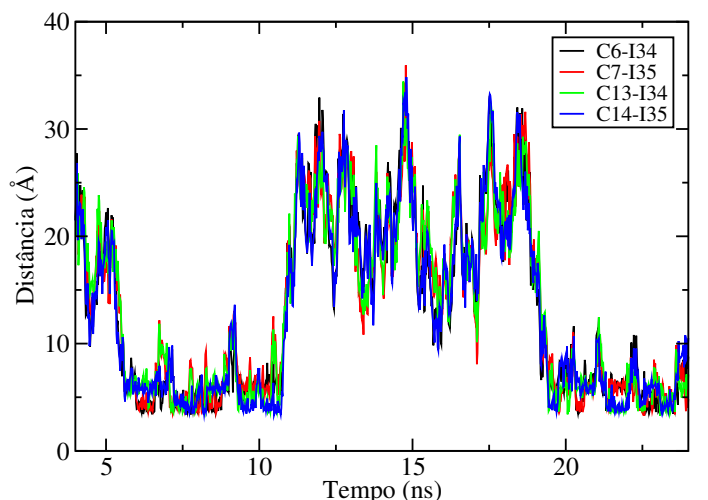

(a)

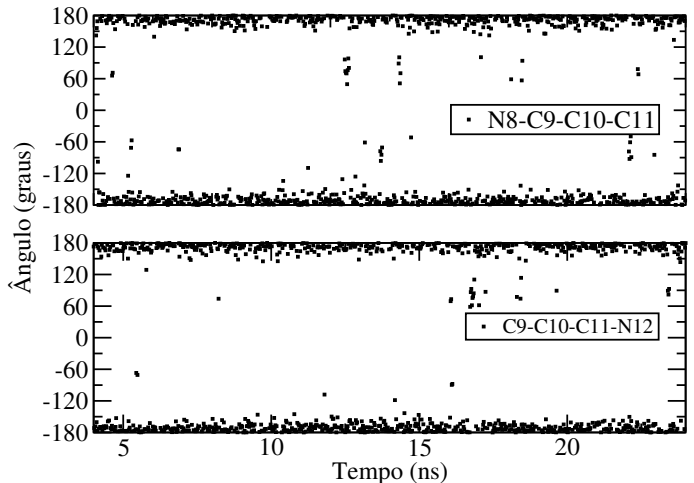

(b)

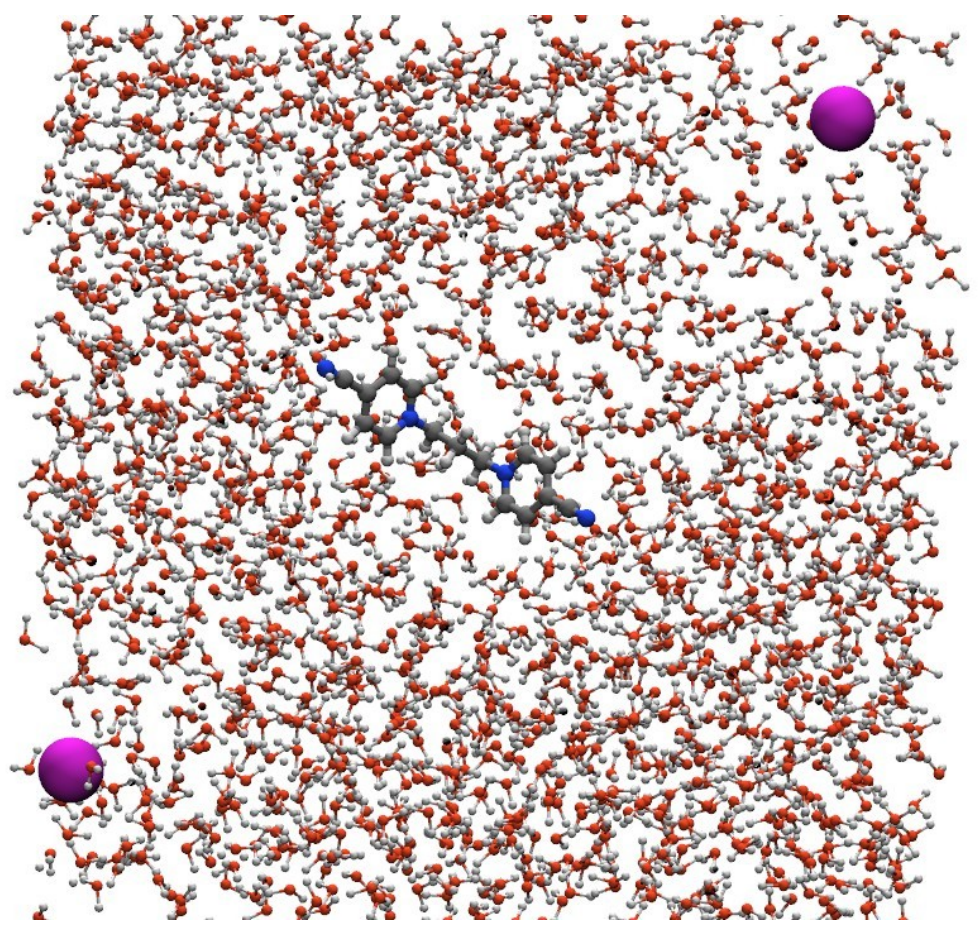

(c)

Figura 5.20: Dinâmica molecular clássica do complexo $C_{3} b i s(4 C P)^{2+}+2 I^{-}$em água: (a) evolução temporal da distâncias C-I; (b) evolução dos diedros da cadeia carbônica; (c) figura ilustrando a dissociação do complexo. 
não são observadas conformações semelhantes a estrutura IV. Isto deixa evidente que a interação com os contra-íons $I^{-}$são essenciais para as mudanças conformacionais observadas em acetonitrila.

Os resultados corroboram com as observações experimentais [35], que mostram a supressão da banda de absorção quando se adiciona água na solução contendo o complexo em acetonitrila.

\subsubsection{Complexo formado pelo $C_{8} b i s(4 C P)^{2+} \operatorname{com} 2 \mathbf{I}^{-}$}

A família de derivados bispiridínicos são formados por sistemas que podem ser sintetizados com diferentes tamanhos de cadeia carbônica. No caso do $C_{8} b i s(4 C P)^{2+}$ essa cadeia é formada por 8 carbonos. O mesmo campo de força usado para o $C_{3} b i s(4 C P)^{2+}$ foi usado para realizar a dinâmica do complexo $C_{8} b i s(4 C P)^{2+}$ com $2 I^{-}$em acetonitrila.

Um dos grandes desafios no estudo desses sistemas é que, com o aumento do número de átomos na cadeia, aumentam também o número de conformações acessíveis ao sistema. Mesmo que procedimentos de busca conformacionais sejam usados para estudar as conformações de mínima energia, é razoável supor um número grande de conformações acessíveis com geometria próximas.

A dinâmica molecular torna-se então um procedimento bastante atrativo para estudar esses sistemas em solução. O mesmo campo de força usado anteriormente pode ser aplicado no estudo do $C_{8} b i s(4 C P)^{2+}$, bastando incluir os parâmetros para os carbonos adicionais da cadeia, que podem ser retirados do campo de força original OPLS para alcanos. A configuração inicial foi uma geometria otimizada onde os ângulos diedrais da cadeia eram iguais a $180^{\circ}$, ou seja, em uma condição onde os anéis estão com máxima distância entre si. Um iodeto foi colocado na proximidade de cada um dos anéis e, com a otimização de geometria, eles ali permaneceram. Uma dinâmica molecular de 24 ns idêntica aquela realizada previamente para o $C_{3}$ bis $(4 C P)^{2+}$ foi realizada.

Após o término da dinâmica, o algoritmo de "clustering" foi usado para identificar as principais conformações amostradas durante a dinâmica. Usando um raio de corte de $1 \AA$ forma identificados 267 clusters. A estrutura mais representativa dos seis 


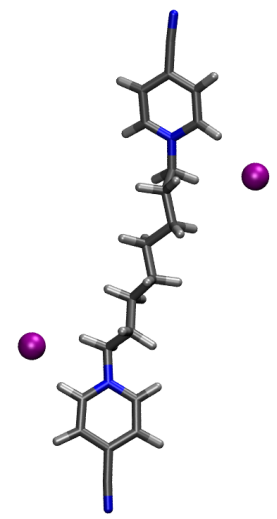

(a) $12 \%$

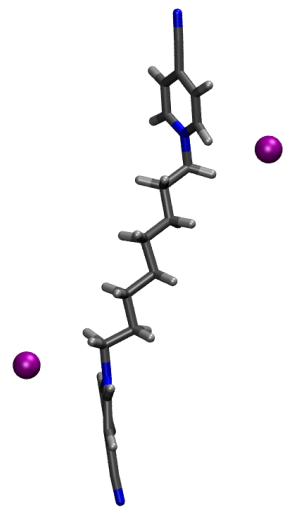

(d) $4 \%$

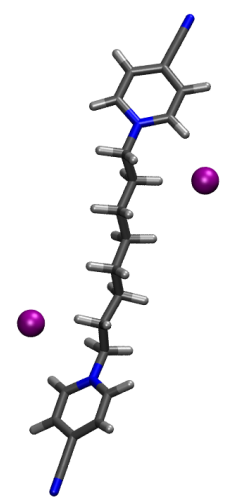

(g) $3 \%$

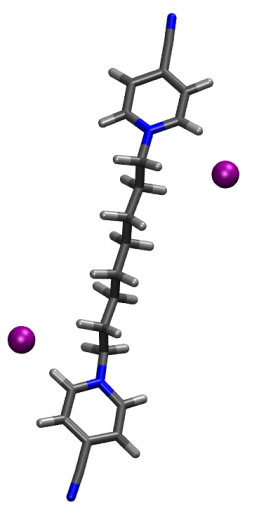

(b) $10 \%$

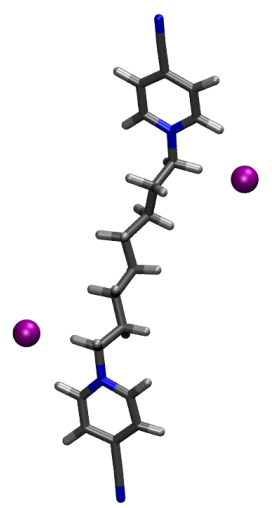

(e) $4 \%$

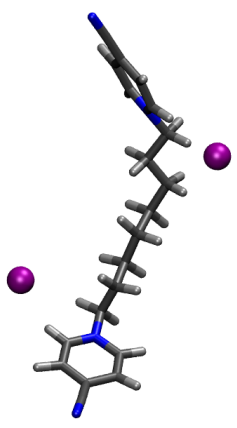

(h) $3 \%$

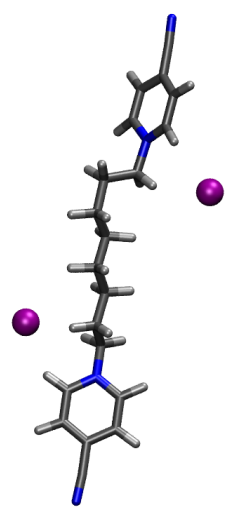

(c) $6 \%$

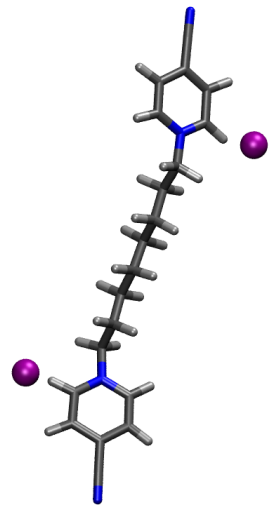

(f) $3 \%$

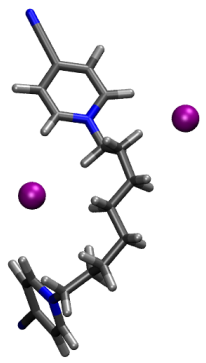

(i) $2 \%$

Figura 5.21: Clusters Moleculares obtidos da dinâmica clássica em acetonitrila. Piridínio: $C_{8}$ bis $(4 C P)^{2+}$ 
clusters mais populados são mostradas na Figura 5.21.

Nota-se que a maior parte das conformações amostradas são aquelas onde os anéis encontram-se afastados. A única conformação onde um dos $I^{-}$interage com os dois anéis é a da Figura 5.21i e responde por apenas $2 \%$ das configurações. 
Capítulo

\section{Considerações finais}

Neste trabalho foram estudados os complexos de transferência de carga formados por iodeto com os derivados piridínicos $C_{4}(4 C P)^{+}$e $C_{3} b i s(4 C P)^{+2}$ em solução. O principal objetivo foi a descrição teórica das propriedades estruturais, eletrônicas e da banda de transferência de carga dos complexos em acetonitrila.

Em relação ao complexo $C_{4}(4 C P)^{+} I^{-}$grande parte das conclusões podem ser tiradas olhando apenas para os resultados da estrutura otimizada. A geometria do complexo é formada pela associação do $I^{-}$ao anel carbono localizado na posição orto do anel. Ao todo 6 excitações de transferência de carga podem ser identificadas e correspondem à transições eletrônicas dos orbitais do $I^{-}$para os orbitais localizados sobre o anel aromático. Sendo assim ocorre o aparecimento de duas bandas no espectro. A banda de maior comprimento de onda é, em geral, a de maior interesse experimental e é composta principalmente por uma transição $\mathrm{HOMO} \rightarrow$ LUMO.

Nossos resultados também mostram que o emprego de funcionais de troca e correlação com correções de longo alcance é essencial para a obtenção de resultados acurados para as energias de excitação dos CTC. Os funcionais CAM-B3LYP e $\omega$ B97XD, associados ao modelo contínuo PCM, conseguiram descrever muito bem o máximo da banda experimental, de $421 \mathrm{~nm}$. O modelo contínuo PCM, entretanto, apresentou algumas limitações em relação a otimização de geometria e descrição das bandas em outros solventes.

O estudo do $C_{3} b i s(4 C P)^{+2}$ mostrou a existência de diferentes estruturas possíveis 
para o complexo. Inicialmente foram estudados complexos formados pela associação com um $I^{-}$. A estrutura II, onde os dois anéis se fecham sobre o $I^{-}$formando uma conformação próxima a proposta experimentalmente, foi a menos favorável energeticamente, além de apresentar excitações cerca de $30 \mathrm{~nm}$ deslocada para maior comprimentos de onda em relação ao máximo da banda experimental.

A estrutura I foi a que apresentou resultados mais consistente com as medidas experimentais, apresentando transições mais intensas e em melhor concordância com o máximo da banda experimental. Nesta estrutura a carga é transferida do iodeto para apenas um dos anéis, resultando em excitações $\mathrm{HOMO} \rightarrow$ LUMO semelhantes ao caso do $C_{4}(4 C P)^{+} I^{-}$. Já na estrutura III apresenta excitações com intensidade quase nulas, já que o $I^{-}$interage em uma região próxima ao plano dos dois anéis.

As estruturas IV e V foram obtidas pela adição de um segundo $I^{-}$as estruturas $\mathbf{I}$ e III, respectivamente. Estas estruturas apresentaram as mesmas energias de excitação em relação ao caso anterior, o que mostra que a adição do segundo contra-íon não afeta o espectro de absorção dos complexos.

A dinâmica molecular clássica do complexo $C_{3}$ bis $(4 C P)^{2+}$ mostrou não haver dissociação do $I^{-}$em acetonitrila, de modo que o complexo alterna entre estruturas IV e V. Nossos resultados mostram que a principal contribuição da banda de transferência de carga vem da excitação $\mathrm{HOMO} \rightarrow$ LUMO das conformações semelhantes a estrutura IV. Apenas um dos $I^{-}$participa da excitação, o que explica a estequiometria 1:1 observada experimentalmente.

A dinâmica molecular clássica também mostrou a dissociação do complexo em água, em concordância com os resutados experimentais que mostram a supressão da banda de transferência de carga neste solvente. O campo de força refinado neste trabalho abre a perspectiva para o estudo de um grande número de compostos pertencentes a família dos derivados piridínicos. Neste sentido podem se explorados, por exemplo, os compostos obtidos pelo aumento da cadeia da cadeia carbônica como o $C_{8} b i s(4 C P)^{2+}$, além dos compostos obtidos pela modificação dos substituintes do anel. O estudo do comportamento dos complexos de transferência de carga nos demais solventes, bem como misturas binárias, são também perspectivas a serem exploradas futuramente. 


\section{Referências Bibliográficas}

[1] K. Müller-Dethlefs e P. Hobza, "Noncovalent interactions: a challenge for experiment and theory," Chemical Reviews, vol. 100, p. 143, 2000.

[2] J. K. Kochi, "Charge-transfer excitation of molecular complexes in organic and organometallic chemistry," Pure and Applied Chemistry, vol. 63, p. 255, 1991.

[3] D. L. A. de Faria e P. S. Santos, "Complexos Moleculares: alguns aspectos históricos," Química Nova, vol. 16, p. 449, 1993.

[4] H. A. Benesi e J. H. Hildebrand, "A spectrophotometric investigation of the interaction of iodine with aromatic hydrocarbons," Journal of the American Chemical Society, vol. 71, p. 2703, 1949.

[5] R. S. Mulliken, "Molecular compounds and their spectra. II," Journal of the American Chemical Society, vol. 74, p. 811, 1952.

[6] K. P. Goetz, D. Vermeulen, M. E. Payne, C. Kloc, L. E. McNeil e O. D. Jurchescu, "Charge-transfer complexes: new perspectives on an old class of compounds," Journal of Materials Chemistry C, vol. 2, p. 3065, 2014.

[7] E. M. Kosower, "Reactions Through Charge-Transfer Complexes," in Progress in Physical Organic Chemistry (S. G. Cohen, A. Streitwieser e R. W. Taft, eds.), vol. 3, p. 81, John Wiley and Sons, 1965.

[8] P. Pal, S. Bhattacharya, A. K. Mukherjee e D. C. Mukherjee, "Absorption spectrometric and thermodynamic study of charge transfer complexes of menadione 
(vitamin K3) with a series of phenols," Spectrochimica Acta-Part A: Molecular and Biomolecular Spectroscopy, vol. 61, p. 879, 2005.

[9] A. Saha e A. K. Mukherjee, "Absorption spectrometric study of charge-transfer complex formation between 4-acetamidophenol (paracetamol) and a series of quinones including vitamin K-3," Spectrochimica Acta - Part A: Molecular and Biomolecular Spectroscopy, vol. 60, p. 1731, 2004.

[10] H. F. Askal, "Spectrophotometric study of the charge-transfer complexes of some pharmaceutical butyrophenones," Talanta, vol. 44, p. 1749, 1997.

[11] H. Duymus, M. Arslan, M. Kucukislamoglu e M. Zengin, "Charge transfer complex studies between some non-steroidal anti-inflammatory drugs and $\pi$-electron acceptors," Spectrochimica Acta - Part A: Molecular and Biomolecular Spectroscopy, vol. 65, p. 1120, 2006.

[12] O. D. Parashchuk, A. Y. Sosorev, V. V. Bruevich e D. Y. Paraschuk, "Threshold formation of an intermolecular charge transfer complex of a semiconducting polymer," Jetp Letters, vol. 91, p. 351, 2010.

[13] J. B. Torrance, "Difference between metallic and insulating salts of tetracyanoquinodimethane (TCNQ) - how to design an organic metal," Accounts of Chemical Research, vol. 12, p. 79, 1979.

[14] G. J. Meyer, "Molecular approaches to solar energy conversion with coordination compounds anchored to semiconductor surfaces," Inorganic Chemistry, vol. 44, p. $6852,2005$.

[15] C. Deibel, T. Strobel e V. Dyakonov, "Role of the charge transfer state in organic donor-acceptor solar cells," Advanced Materials, vol. 22, p. 4097, 2010.

[16] J. L. Bredas, J. E. Norton, J. Cornil e V. Coropceanu, "Molecular understanding of organic solar cells: the challenges," Accounts of Chemical Research, vol. 42, p. 1691, 2009.

[17] A. Rouhollahi e M. Shamsipur, "Triiodide PVC membrane electrode based on a charge-transfer complex of iodine with 2,4,6,8-tetraphenyl-2,4,6,8tetraazabicyclo 3.3.0 octane," Analytical Chemistry, vol. 71, p. 1350, 1999. 
[18] S. Sadeghi e G. R. Dashti, "Triiodide PVC membrane electrodes based on chargetransfer complexes," Analytical Chemistry, vol. 74, p. 2591, 2002.

[19] E. M. Kosower, "Additions to pyridinium rings. I. 1-methylpyridinium iodide," Journal of the American Chemical Society, vol. 77, p. 3883, 1955.

[20] E. M. Kosower e P. E. Klinedinst, "Additions to pyridinium rings. II. Chargetransfer complexes as intermediates," Journal of the American Chemical Society, vol. 78, p. 3493, 1956.

[21] E. M. Kosower, "The art of discovery in biophysical organic chemistry," Canadian Journal of Chemistry-Revue Canadienne De Chimie, vol. 83, p. 1207, 2005.

[22] E. M. Kosower, "The effect of solvent on spectra. I. A new empirical measure of solvent polarity - Z-values," Journal of the American Chemical Society, vol. 80, p. 3253, 1958.

[23] M. Y. Lui, L. Crowhurst, J. P. Hallett, P. a. Hunt, H. Niedermeyer e T. Welton, "Salts dissolved in salts: ionic liquid mixtures," Chemical Science, vol. 2, p. 1491, 2011.

[24] P. Chatterjee, A. K. Laha e S. Bagchi, "Preferential solvation in mixed binary solvents: ultraviolet?visible spectroscopy of N-alkylpyridinium lodides in mixed solvents containing cyclic ethers," 1992.

[25] P. Chatterjee e S. Bagchi, "Preferential solvation of a dipolar solute in mixed binary solvent: A study of UV-visible spectroscopy," Journal of Physical Chemistry, vol. 95, p. 3311, 1991.

[26] P. Chatterjee, K. Medda e S. Bagchi, "A study of preferential solvation of Nalkyl pyridinium iodides in mixed solvents containing acetonitrile," Journal of Solution Chemistry, vol. 20, p. 249, 1991.

[27] P. Chatterjee e S. Bagchi, "Preferential solvation in mixed binary solvents by ultraviolet-visible spectroscopy: N-ethyl-4-cyanopyridinium iodide in alcoholacetone mixtures," Journal of the Chemical Society, Faraday Transactions, vol. 87, p. $587,1991$. 
[28] A. K. Laha, P. K. Das, D. Banerjee e S. Bagchi, "UV-Vis spectroscopic study of preferential solvation in mixed binary solvents at various temperatures," Journal of the Chemical Society, Faraday Transactions, vol. 92, p. 1499, 1996.

[29] A. K. Laha, P. K. Das e S. Bagchi, "Study of preferential solvation in mixed binary solvent as a function of solvent composition and temperature by UV-Vis spectroscopic method," Journal of Physical Chemistry A, vol. 106, p. 3230, 2002.

[30] M. Politi, I. M. Cuccovia, H. Chaimovich, M. Almeida, J. B. S. Bonilha e F. H. Quina, "Effect of hexadecyltrimethylammonium bromide on hydrolysis of Nalkyl-4-cyanopyridinium ions," Tetrahedron Letters, vol. 19, p. 115, 1978.

[31] N. Hioka, M. J. Politi e H. Chaimovich, "Kinetic demonstration of premicellar aggregation - The alkaline-hydrolysis of N-hexadecyl-4-cyanopyridinium bromide," Tetrahedron Letters, vol. 30, p. 1051, 1989.

[32] M. J. Politi e H. Chaimovich, "Micellar effects on the alkaline-hydrolysis of Nalkyl-4-cyanopyridinium ions - An example of micelle-induced regiochemical selectivity," Journal of Physical Organic Chemistry, vol. 4, p. 207, 1991.

[33] C. Tanford, The hydrophobic effect: formation of micelles and biological membranes. Wiley-Interscience, 1973.

[34] S. Fiori, Estudos da reação de hidrólise alcalina de derivados N-alquil-2azidopiridínios e de derivados $N$-alquil-4-cianopiridínios . Tese (Doutorado), Universidade Estadual de Maringá, 2011.

[35] F. R. Carvalho, Caracterização de Complexos de Transferência de Carga entre ions Derivados de N,N'-alquildiil-bis(piridinio substituídos) com Iodeto. Dissertação (Mestrado), Universidade Estadual de Maringá, 2011.

[36] E. M. Kosower, "Additions to pyridinium rings. III. Chemical and biochemical implications of charge-transfer complex intermediates," Journal of the American Chemical Society, vol. 78, p. 3497, 1956.

[37] E. M. Kosower e J. C. Burbach, "Equilibrium constants for pyridinium iodide charge-transfer complex formation," Journal of the American Chemical Society, vol. 78 , p. 5838, 1956. 
[38] E. M. Kosower, "The effect of solvent on charge-transfer complex spectra," Journal of the American Chemical Society, vol. 78, p. 5700, 1956.

[39] E. M. Kosower, "The effect of solvent on spectra. II. Correlation of spectral absorption data with Z-values," Journal of the American Chemical Society, vol. 80, p. 3261, 1958.

[40] E. M. Kosower, J. A. Skorcz, W. M. Schwarz e J. W. Patton, "Pyridinium complexes. I. The significance of the 2 nd charge-transfer band of pyridinium iodides," Journal of the American Chemical Society, vol. 82, p. 2188, 1960.

[41] M. Ito, P. C. Huang e E. M. Kosower, "Electronic absorption spectra of iodoand bromomethanes," Transactions of the Faraday Society, vol. 57, p. 1662, 1961.

[42] E. M. Kosower, G. S. Wu e T. S. Sorensen, "Effect of solvent on spectra. VI. Detection of solvent effect on molecular conformation or shape through Z-values," Journal of the American Chemical Society, vol. 83, p. 3147, 1961.

[43] E. M. Kosower, D. Hofmann e K. Wallenfels, "Pyridinium complexes. IV. Effect of N-substituents on position of iodide charge-transfer band," Journal of the American Chemical Society, vol. 84, p. 2755, 1962.

[44] J. N. Canongia Lopes, J. Deschamps e A. A. H. Pádua, "Modeling Ionic Liquids Using a Systematic All-Atom Force Field," Journal of Physical Chemistry B, vol. 108, p. 2038, 2004.

[45] C. Cadena, Q. Zhao, R. Q. Snurr e E. J. Maginn, "Molecular Modeling and Experimental Studies of the Thermodynamic and Transport Properties of PyridiniumBased Ionic Liquids," Journal of Physical Chemistry B, vol. 110, p. 2821, 2006.

[46] J. N. Canongia Lopes e A. A. H. Pádua, "Molecular force field for ionic liquids III: Imidazolium, pyridinium, and phosphonium cations; chloride, bromide, and dicyanamide anions," Journal of Physical Chemistry B, vol. 110, p. 19586, 2006.

[47] O. Borodin, "Polarizable force field development and molecular dynamics simulations of ionic liquids.," Journal of physical chemistry. B, vol. 113, p. 11463, 2009. 
[48] S. V. Sambasivarao e O. Acevedo, "Development of OPLS-AA force field parameters for 68 unique ionic liquids," Journal of Chemical Theory and Computation, vol. 5, p. 1038, 2009.

[49] J. N. Canongia Lopes e A. A. H. Pádua, "CL\&P: A generic and systematic force field for ionic liquids modeling," Theoretical Chemistry Accounts, vol. 131, p. 1129, 2012.

[50] O. Acevedo, "Simulating Chemical Reactions in Ionic Liquids Using QM/MM Methodology," Journal of Physical Chemistry A, vol. 118, p. 11653, 2014.

[51] B. Tong, Q.-S. Liu, Z.-C. Tan e U. Welz-Biermann, "Thermochemistry of Alkyl Pyridinium Bromide Ionic Liquids: Calorimetric Measurements and Calculations," Journal of Physical Chemistry A, vol. 114, p. 3782, 2010.

[52] K. C. Lethesh, K. V. Hecke, L. V. Meervelt, P. Nockemann, B. Kirchner, S. Zahn, T. N. Parac-Vogt, W. Dehaen e K. Binnemans, "Nitrile-Functionalized Pyridinium, Pyrrolidinium, and Piperidinium Ionic Liquids," Journal of Physical Chemistry B, vol. 115, p. 8424, 2011.

[53] B. Bittner, R. J. Wrobel e E. Milchert, "Physical properties of pyridinium ionic liquids," Journal of Chemical Thermodynamics, vol. 55, p. 159, 2012.

[54] T. Ogura, N. Akai, K. Shibuya e A. Kawai, "Charge-transfer electronic absorption spectra of 1-ethylpyridinium cation and halogen anion pairs in dichloromethane and as neat ionic liquids," Journal of Physical Chemistry B, vol. 117, p. $8547,2013$.

[55] C. Fernandez, V. G. Toscano, H. Chaimovich, M. J. Politi e N. Hioka, "Reactions of 1,w-bis(2-bromopyridinium)alkanes with hydroxide ion in aqueous solutions," Journal of Physical Organic Chemistry, vol. 11, p. 25, 1998.

[56] M. d. G. N. Corrêa, M. J. Politi e N. Hioka, "Reactions of 1,w-bis(2bromopyridinium)alkanes with azide ions: Charge effect and intermediates," Journal of Physical Organic Chemistry, vol. 12, p. 837, 1999. 
[57] S. Fiori, I. T. A. Shuquel, I. N. da Silva, L. H. Catalani, H. Chaimovich, E. Meyer e N. Hioka, "Characterization Studies of 1-(4-Cyano-2-oxo-1,2dihydro-1-pyridyl)3-(4-cyano-1,2-dihydro-1-pyridyl) propane formed from the reaction of hydroxide ion with 1,3-bis-(4-cyanopyridinium)propane," Journal of the Brazilian Chemical Society, vol. 22, p. 1644, 2011.

[58] S. Fiori, F. R. de Carvalho, A. L. Tessaro, E. Meyer, W. Caetano, M. J. Politi e N. Hioka, "Kinetic Study of the Alkaline Hydrolysis of 1,n-Bis(4cyanopyridinium)alkanes: Charge Density and New Conformational Effects on the Reactivity of 1,3-Bis(4-cyanopyridinium)propane," International Journal of Chemical Kinetics, vol. 45, p. 478, 2013.

[59] Z. D. Hill e P. MacCarthy, "Novel approach to Job's method: An undergraduate experiment," Journal of Chemical Education, vol. 63, p. 162, 1986.

[60] V. M. S. Gil e N. C. Oliveira, "On the use of the method of continuous variations," Journal of Chemical Education, vol. 67, p. 473, 1990.

[61] P. Thordarson, "Determining association constants from titration experiments in supramolecular chemistry," Chem. Soc. Rev., vol. 40, p. 1305, 2011.

[62] J. S. Renny, L. L. Tomasevich, E. H. Tallmadge e D. B. Collum, "Method of Continuous Variations: Applications of Job Plots to the Study of Molecular Associations in Organometallic Chemistry," Angewandte Chemie International Edition, vol. 52, p. 11998, 2013.

[63] E. A. Boucher e C. C. Mollett, "Coloured and colourless charge-transfer complexes of small and polymeric quaternary pyridinium bromides," Journal of the Chemical Society, Faraday Transactions 1, vol. 78, p. 1401, 1982.

[64] A. Ray, "Effects of temperature and solvent polarity on the interionic chargetransfer interactions in alkylpyridinium bromide," Journal of the American Chemical Society, vol. 93, p. 7146, 1971.

[65] S. P. A. Sauer, M. Schreiber, M. R. Silva-Junior e W. Thiel, "Benchmarks for electronically excited states: A comparison of noniterative and iterative triples 
corrections in linear response coupled cluster methods: CCSDR(3) versus CC3," Journal of Chemical Theory and Computation, vol. 5, p. 555, 2009.

[66] K. Andersson, P. A. Malmqvist e B. O. Roos, "2nd-order perturbation-theory with a complete active space self-consistent field reference function," Journal of Chemical Physics, vol. 96, p. 1218, 1992.

[67] K. Burke, "Perspective on density functional theory," Journal of Chemical Physics, vol. 136, p. 150901, 2012.

[68] W. Kohn, "Nobel lecture: Electronic structure of matter-wave functions and density functionals," Reviews of Modern Physics, vol. 71, p. 1253, 1999.

[69] A. J. Cohen, P. Mori-Sanchez e W. Yang, "Challenges for density functional theory," Chemical Reviews, vol. 112, p. 289, 2012.

[70] S. N. Steinmann, C. Piemontesi, A. Delacht e C. Corminboeuf, "Why are the interaction energies of charge-transfer complexes challenging for DFT?," Journal of Chemical Theory and Computation, vol. 8, p. 1629, 2012.

[71] J. Arago, J. C. Sancho-Garcia, E. Orti e D. Beljonne, "Ab initio modeling of donor-acceptor interactions and charge-transfer excitations in molecular complexes: The case of terthiophene-tetracyanoquinodimethane," Journal of Chemical Theory and Computation, vol. 7, p. 2068, 2011.

[72] A. Dreuw e M. Head-Gordon, "Failure of time-dependent density functional theory for long-range charge-transfer excited states: The zincbacteriochlorinbacterlochlorin and bacteriochlorophyll-spheroidene complexes," Journal of the American Chemical Society, vol. 126, p. 4007, 2004.

[73] J. D. Chai e M. Head-Gordon, "Long-range corrected hybrid density functionals with damped atom-atom dispersion corrections," Physical Chemistry Chemical Physics, vol. 10, p. 6615, 2008.

[74] A. Dreuw, J. L. Weisman e M. Head-Gordon, "Long-range charge-transfer excited states in time-dependent density functional theory require non-local exchange," Journal of Chemical Physics, vol. 119, 2003. 
[75] H. Iikura, T. Tsuneda, T. Yanai e K. Hirao, "A long-range correction scheme for generalized-gradient-approximation exchange functionals," Journal of Chemical Physics, vol. 115, p. 3540, 2001.

[76] T. Yanai, D. P. Tew e N. C. Handy, "A new hybrid exchange-correlation functional using the coulomb-attenuating method (CAM-B3LYP)," Chemical Physics Letters, vol. 393, p. 51, 2004.

[77] T. Stein, L. Kronik e R. Baer, "Reliable prediction of charge transfer excitations in molecular complexes using time-dependent density functional theory," Journal of the American Chemical Society, vol. 131, p. 2818, 2009.

[78] M. Born, "Volumes and hydration warmth of ions," Zeitschrift Fur Physik, vol. 1, p. $45,1920$.

[79] L. Onsager, "Electric moments of molecules in liquids," Journal of the American Chemical Society, vol. 58, p. 1486, 1936.

[80] J. G. Kirkwood, "On the theory of dielectric polarization," Journal of Chemical Physics, vol. 4, p. 592, 1936.

[81] O. Tapia e O. Goscinski, "Self-consistent reaction field theory of solvent effects," Molecular Physics, vol. 29, p. 1653, 1975.

[82] J. Rivail e D. Rinaldi, "A quantum chemical approach to dielectric solvent effects in molecular liquids," Chemical Physics, vol. 18, p. 233, 1976.

[83] S. Miertus, E. Scrocco e J. Tomasi, "Electrostatic interaction of a solute with a continuum - A direct utilization of ab initio molecular potentials for the prevision of solvent effects," Journal of Chemical Physics, vol. 55, p. 117, 1981.

[84] J. Tomasi e M. Persico, "Molecular interactions in solution - An overview of methods based on continuous distributions of the solvent," Chemical Reviews, vol. 94, p. 2027, 1994.

[85] E. Cances, B. Mennucci e J. Tomasi, "A new integral equation formalism for the polarizable continuum model: Theoretical background and applications to isotropic and anisotropic dielectrics," Journal of Chemical Physics, vol. 107, p. 3032, 1997. 
[86] J. Tomasi, B. Mennucci e R. Cammi, "Quantum mechanical continuum solvation models," Chemical reviews, vol. 105, p. 2999, 2005.

[87] M. P. Allen e D. J. Tildesley, Computer simulation of liquids. Oxford university press, 1989.

[88] W. F. van Gunsteren e H. J. C. Berendsen, "Computer Simulation of Molecular Dynamics: Methodology, Applications, and Perspectives in Chemistry," Angewandte Chemie International Edition in English, vol. 29, p. 992, 1990.

[89] M. J. Field, A practical introduction to the simulation of molecular systems. Cambridge University Press, 1999.

[90] M. Karplus e J. A. McCammon, "Molecular dynamics simulations of biomolecules," Nature Structural Biology, vol. 9, p. 646, 2002.

[91] G. Sutmann, "Classical Molecular Dynamics," in NIC Series Volume 10. Quantum Simulations of Complex Many-Body Systems: From Theory to Algorithms (J. Grotendorst, D. Marx e A. Muramatsu, eds.), p. 211, John von Neumann Institute for Computing, 2002.

[92] D. C. Rapaport, The art of molecular dynamics simulation. Cambridge university press, 2004.

[93] M. P. Allen, "Introduction to molecular dynamics simulation," in NIC Series Volume 23. Computational Soft Matter: From Synthetic Polymers to Proteins (N. Attig, K. Binder, H. G. uller e K. Kremer, eds.), p. 1, John von Neumann Institute for Computing, 2004.

[94] D. Marx e J. Hutter, Ab initio molecular dynamics: basic theory and advanced methods. Cambridge University Press, 2009.

[95] Car e Parrinello, "Unified approach for molecular dynamics and densityfunctional theory.," Physical review letters, vol. 55, p. 2471, 1985.

[96] M. C. Payne, J. D. Joannopoulos, D. C. Allan, M. P. Teter e D. H. Vanderbilt, "Molecular Dynamics and ab initio Total Energy Calculations," Physical Review Letters, vol. 56, p. 2656, 1986. 
[97] M. J. Field, P. A. Bash e M. Karplus, "A combined quantum mechanical and molecular mechanical potential for molecular dynamics simulations," Journal of Computational Chemistry, vol. 11, p. 700, 1990.

[98] H. Lin e D. G. Truhlar, "QM/MM: What have we learned, where are we, and where do we go from here?," Theoretical Chemistry Accounts, vol. 117, p. 185, 2007.

[99] H. M. Senn e W. Thiel, "QM/MM Methods for Biomolecular Systems," Angewandte Chemie International Edition, vol. 48, p. 1198, 2009.

[100] J. Gao e M. A. Thompson, eds., Combined Quantum Mechanical and Molecular Mechanical Methods, vol. 712 of ACS Symposium Series. American Chemical Society, 1998.

[101] A. Warshel e M. Levitt, "Theoretical studies of enzymic reactions: dielectric, electrostatic and steric stabilization of the carbonium ion in the reaction of lysozyme.," Journal of molecular biology, vol. 103, p. 227, 1976.

[102] M. Karplus, "Development of Multiscale Models for Complex Chemical Systems: From $\mathrm{H}+\mathrm{H}_{2}$ to Biomolecules (Nobel Lecture)," Angewandte Chemie International Edition, vol. 53, p. 9992, 2014.

[103] M. Levitt, "Birth and Future of Multiscale Modeling for Macromolecular Systems (Nobel Lecture)," Angewandte Chemie International Edition, vol. 53, p. 10006, 2014.

[104] A. Warshel, "Multiscale Modeling of Biological Functions: From Enzymes to Molecular Machines (Nobel Lecture)," Angewandte Chemie International Edition, vol. 53, p. 10020, 2014.

[105] E. Brunk e U. Rothlisberger, "Mixed Quantum Mechanical/Molecular Mechanical Molecular Dynamics Simulations of Biological Systems in Ground and Electronically Excited States," Chemical Reviews, vol. 115, p. 6217, 2015.

[106] H. M. Senn e W. Thiel, "QM/MM studies of enzymes," Current Opinion in Chemical Biology, vol. 11, p. 182, 2007. 
[107] J. T. Blair, K. Krogh-Jespersen e R. M. Levy, "Solvent effects on optical absorption spectra: the ${ }^{1} A_{1} \rightarrow{ }^{1} A_{2}$ transition of formaldehyde in water," Journal of the American Chemical Society, vol. 111, p. 6948, 1989.

[108] J. T. Blair, R. M. Levy e K. Krogh-Jespersen, "Molecular mechanics parameters for electronically excited states: The (n, $\left.\pi^{*}\right)$ singlet state of formaldehyde," Chemical Physics Letters, vol. 166, p. 429, 1990.

[109] J. Zeng, J. S. Craw, N. S. Hush e J. R. Reimers, "Solvent effects on molecular spectra. I. Normal pressure and temperature Monte Carlo simulations of the structure of dilute pyrimidine in water," Journal of Chemical Physics, vol. 99, p. 1482, 1993.

[110] J. Zeng, N. S. Hush e J. R. Reimers, "Solvent effects on molecular spectra. II. Simulations of hydrated clusters and dilute solutions of pyrimidine in its lowest (n, $\left.\pi^{*}\right)$ singlet excited state," Journal of Chemical Physics, vol. 99, p. 1496, 1993.

[111] J. Zeng, N. S. Hush e J. R. Reimers, "Solvent effects on molecular spectra. III. Absorption to and emission from the lowest singlet $\left(\mathrm{n}, \pi^{*}\right)$ state of dilute pyrimidine in water," Journal of Chemical Physics, vol. 99, p. 1508, 1993.

[112] J. Zeng, J. S. Craw, N. S. Hush e J. R. Reimers, "Solvent Effects on Molecular and Ionic Spectra. IV. Photochemistry of $\mathrm{Fe}^{2+}(\mathrm{H} 2 \mathrm{O})_{6}$ in Water Revisited: Possible Mechanisms for the Primary Absorption Process Leading to Electron Ejection," Journal of Physical Chemistry, vol. 98, p. 11075, 1994.

[113] K. Coutinho, S. Canuto e M. C. Zerner, "Calculation of the absorption spectrum of benzene in condensed phase. A study of the solvent effects," International Journal of Quantum Chemistry, vol. 65, p. 885, 1997.

[114] K. Coutinho e S. Canuto, "Solvent effects from a sequential Monte Carlo - Quantum mechanical approach," in Advances in Quantum Chemistry, Volume 28 Recent Advances in Computational Chemistry (P. O. Lowdin, J. R. Sabin, M. C. Zerner, J. Karwowski e M. Karelson, eds.), p. 89, Academic Press Inc, 1997. 
[115] H. C. Geog e S. Canuto, "Métodos Híbridos para Modelagem do Ambiente Molecular," in Métodos de Química Teórica e Modelagem Molecular (N. H. Morgon e K. Coutinho, eds.), p. 301, Editora Livraria da Física, 2007.

[116] K. Coutinho, R. Rivelino, H. C. Georg e S. Canuto, "The Sequential QM/MM Method and its Applications to Solvent Effects in Electronic and Structural Properties of Solutes," in Solvation Effects on Molecules and Biomolecules (S. Canuto, ed.), p. 159, Springer Netherlands, 2008.

[117] S. Canuto e K. Coutinho, "From hydrogen bond to bulk: Solvation analysis of the $n \longrightarrow \pi^{*}$ transition of formaldehyde in water," International Journal of Quantum Chemistry, vol. 77, p. 192, 2000.

[118] T. Malaspina, K. Coutinho e S. Canuto, "Ab initio calculation of hydrogen bonds in liquids: A sequential Monte Carlo quantum mechanics study of pyridine in water," Journal of Chemical Physics, vol. 117, p. 1692, 2002.

[119] K. J. de Almeida, K. Coutinho, W. B. de Almeida, W. R. Rocha e S. Canuto, "A Monte Carlo-quantum mechanical study of the solvatochromism of pyrimidine in water and in carbon tetrachloride," Physical Chemistry Chemical Physics, vol. 3, p. $1583,2001$.

[120] W. R. Rocha, K. J. De Almeida, K. Coutinho e S. Canuto, "The electronic spectrum of N-methylacetamide in aqueous solution: a sequential Monte Carlo/quantum mechanical study," Chemical Physics Letters, vol. 345, p. 171, 2001.

[121] W. R. Rocha, V. M. Martins, K. Coutinho e S. Canuto, "Solvent effects on the electronic absorption spectrum of formamide studied by a sequential Monte Carlo/quantum mechanical approach," Theoretical Chemistry Accounts, vol. 108, p. 31, 2002.

[122] M. Z. Hernandes, R. Longo, K. Coutinho e S. Canuto, "Solute relaxation on the solvatochromism of ortho-betaine dyes. A sequential Monte Carlo/quantum mechanics study," Physical Chemistry Chemical Physics, vol. 6, p. 2088, 2004.

[123] H. C. Georg, K. Coutinho e S. Canuto, "A sequential Monte Carlo quantum mechanics study of the hydrogen-bond interaction and the solvatochromic shift of 
the n-pi(*) transition of acrolein in water," Journal of Chemical Physics, vol. 123, p. $124307,2005$.

[124] V. Ludwig, K. Coutinho e S. Canuto, "A Monte Carlo-quantum mechanics study of the lowest $n-\pi *$ and $\pi-\pi *$ states of uracil in water," Physical Chemistry Chemical Physics, vol. 9, p. 4907, 2007.

[125] R. C. Barreto, K. Coutinho, H. C. Georg e S. Canuto, "Combined Monte Carlo and quantum mechanics study of the solvatochromism of phenol in water. The origin of the blue shift of the lowest $\pi-\pi *$ transition.," Physical chemistry chemical physics : PCCP, vol. 11, p. 1388, 2009.

[126] J. Kongsted, B. Mennucci, K. Coutinho e S. Canuto, "Solvent effects on the electronic absorption spectrum of camphor using continuum, discrete or explicit approaches," Chemical Physics Letters, vol. 484, p. 185, 2010.

[127] A. C. Borin, L. Serrano-Andrés, V. Ludwig e S. Canuto, "Theoretical absorption and emission spectra of 1H- and 2H-benzotriazole," Physical Chemistry Chemical Physics, vol. 5, p. 5001, 2003.

[128] K. Coutinho e S. Canuto, "The sequential Monte Carlo-quantum mechanics methodology. Application to the solvent effects in the Stokes shift of acetone in water," Journal of Molecular Structure: THEOCHEM, vol. 632, p. 235, 2003.

[129] R. Rivelino, K. Coutinho e S. Canuto, "A Monte Carlo-quantum mechanics study of the solvent-induced spectral shift and the specific role of hydrogen bonds in the conformational equilibrium of furfural in water," Journal of Physical Chemistry B, vol. 106, p. 12317, 2002.

[130] E. Fileti, P. Chaudhuri e S. Canuto, "Relative strength of hydrogen bond interaction in alcohol-water complexes," Chemical Physics Letters, vol. 400, p. 494, 2004.

[131] E. E. Fileti e S. Canuto, "Ab initio NMR study of the isomeric hydrogenbonded methanol-water complexes," International Journal of Quantum Chemistry, vol. 102, p. 554, 2005. 
[132] E. E. Fileti, H. C. Georg, K. Coutinho e S. Canuto, "Isotropic and anisotropic NMR chemical shifts in liquid water: A sequential QM/MM study," Journal of the Brazilian Chemical Society, vol. 18, p. 74, 2007.

[133] T. L. Fonseca, K. Coutinho e S. Canuto, "The isotropic nuclear magnetic shielding constants of acetone in supercritical water: A sequential Monte Carlo/quantum mechanics study including solute polarization," Journal of Chemical Physics, vol. 129, 2008.

[134] R. M. Gester, C. Bistafa, H. C. Georg, K. Coutinho e S. Canuto, "Theoretically describing the O-17 magnetic shielding constant of biomolecular systems: uracil and 5-fluorouracil in water environment," Theoretical Chemistry Accounts, vol. 133, 2013.

[135] R. M. Gester, C. Bistafa, H. C. Georg, K. Coutinho e S. Canuto, "Theoretically describing the $17 \mathrm{O}$ magnetic shielding constant of biomolecular systems: Uracil and 5-fluorouracil in water environment," Theoretical Chemistry Accounts, vol. 133, p. 1, 2014.

[136] R. C. Guedes, K. Coutinho, B. J. C. Cabral, S. Canuto, C. F. Correia, R. M. B. dos Santos e J. A. M. Simoes, "Solvent effects on the energetics of the phenol $\mathrm{O}-\mathrm{H}$ bond: Differential solvation of phenol and phenoxy radical in benzene and acetonitrile," Journal of Physical Chemistry A, vol. 107, p. 9197, 2003.

[137] K. Coutinho, B. C. Cabral e S. Canuto, "Can larger dipoles solvate less? Solutesolvent hydrogen bond and the differential solvation of phenol and phenoxy," Chemical Physics Letters, vol. 399, p. 534, 2004.

[138] E. Fileti, K. Coutinho e S. Canuto, "Is there a favorite isomer for hydrogenbonded methanol in water?," Advances in Quantum Chemistry, Vol 47, vol. 47, p. 51, 2004.

[139] D. L. Silva, K. Coutinho e S. Canuto, "Electronic spectroscopy of biomolecules in solution: fluorescein dianion in water," Molecular Physics, vol. 108, p. 3125, 2010 . 
[140] P. Jaramillo, K. Coutinho, B. J. C. Cabral e S. Canuto, "Explicit solvent effects on the visible absorption spectrum of a photosynthetic pigment: Chlorophyllc(2) in methanol," Chemical Physics Letters, vol. 516, p. 250, 2011.

[141] M. V. A. Damasceno, B. J. C. Cabral e K. Coutinho, "Structure and electronic properties of hydrated mesityl oxide: A sequential quantum mechanics/molecular mechanics approach," Theoretical Chemistry Accounts, vol. 131, p. 1, 2012.

[142] W. Koch, M. C. Holthausen e M. C. Holthausen, A chemist's guide to density functional theory, vol. 2. Wiley-Vch Weinheim, 2001.

[143] D. S. Sholl e J. A. Steckel, Density Functional Theory: A Practical Introduction. John Wiley \& Sons, Inc., 2009.

[144] E. Engel e R. M. Dreizler, Density Functional Theory: An Advanced Course. Springer, 2011.

[145] E. G. KU e R. M. Dreizler, Density functional theory, vol. 337. Springer Science \& Business Media, 1995.

[146] K. Capelle, "A bird's-eye view of density-functional theory," Brazilian Journal of Physics, vol. 36, p. 1318, 2006.

[147] K. Burke e L. O. Wagner, "DFT in a nutshell," International Journal of Quantum Chemistry, vol. 113, p. 96, 2013.

[148] C. A. Ullrich, Time-dependent density-functional theory: concepts and applications. Oxford University Press, 2011.

[149] M. A. Marques, N. T. Maitra, F. M. Nogueira, E. Gross e A. Rubio, eds., Fundamentals of Time-Dependent Density Functional Theory, vol. 837 of Lecture Notes in Physics. Springer Berlin Heidelberg, 2012.

[150] M. Marques e E. Gross, "Time-Dependent Density Functional Theory," Annual Review of Physical Chemistry, vol. 55, p. 427, 2004.

[151] K. Burke, J. Werschnik e E. K. U. Gross, "Time-dependent density functional theory: Past, present, and future," Journal of Chemical Physics, vol. 123, p. 1, 2005. 
[152] M. Casida e M. Huix-Rotllant, "Progress in Time-Dependent Density-Functional Theory," Annual Review of Physical Chemistry, vol. 63, p. 287, 2012.

[153] C. A. Ullrich e Z.-h. Yang, "A Brief Compendium of Time-Dependent Density Functional Theory," Brazilian Journal of Physics, vol. 44, p. 154, 2014.

[154] P. Hohenberg e W. Kohn, "Inhomogeneous electron gas," Physical Review, vol. 136, p. B864, 1964.

[155] W. Kohn e L. J. Sham, "Self-consistent equations including exchange and correlation effects," Physical Review, vol. 140, p. 1133, 1965.

[156] E. Runge e E. K. U. Gross, "Density-functional theory for time-dependent systems," Physical Review Letters, vol. 52, p. 997, 1984.

[157] M. Born e K. Huang, Dynamical Theory of Crystal Lattices. International series of monographs on physics, Clarendon Press, 1998.

[158] A. Szabo e N. S. Ostlund, Modern quantum chemistry: introduction to advanced electronic structure theory. Courier Dover Publications, 1989.

[159] J. P. Perdew e K. Schmidt, "Jacob's ladder of density functional approximations for the exchange-correlation energy," in International Conference on Density Functional Theory and its Applications to Materials, vol. 577 of Aip Conference Proceedings, p. 1, 2001.

[160] J. P. Perdew, A. Ruzsinszky, L. A. Constantin, J. Sun e G. I. Csonka, "Some Fundamental Issues in Ground-State Density Functional Theory: A Guide for the Perplexed," Journal of Chemical Theory and Computation, vol. 5, p. 902, 2009.

[161] C. T. Lee, W. T. Yang e R. G. Parr, "Development of the Colle-Salvetti correlation-energy formula into a functional of the electron-density," Physical Review B, vol. 37, p. 785, 1988.

[162] J. P. Perdew, J. A. Chevary, S. H. Vosko, K. A. Jackson, M. R. Pederson, D. J. Singh e C. Fiolhais, "Atoms, molecules, solids, and surfaces - applications of 
the generalized gradient approximation for exchange and correlation," Physical Review B, vol. 46, p. 6671, 1992.

[163] J. P. Perdew, "Density-functional approximation for the correlation-energy of the inhomogeneous electron-gas," Physical Review B, vol. 33, p. 8822, 1986.

[164] A. D. Becke, "Density-functional exchange-energy approximation with correct asymptotic-behavior," Physical Review A, vol. 38, p. 3098, 1988.

[165] J. P. Perdew e W. Yue, "Accurate and simple density functional for the electronic exchange energy - generalized gradient approximation," Physical Review B, vol. 33, p. 8800, 1986.

[166] J. P. Perdew, K. Burke e M. Ernzerhof, "Generalized gradient approximation made simple," Physical Review Letters, vol. 77, p. 3865, 1996.

[167] A. D. Becke, "Density-functional thermochemistry. III. The role of exact exchange," Journal of Chemical Physics, vol. 98, p. 5648, 1993.

[168] O. A. Vydrov e G. E. Scuseria, "Assessment of a long-range corrected hybrid functional," Journal of Chemical Physics, vol. 125, 2006.

[169] F. Jensen, Introduction to Computational Chemistry. Wiley, 2007.

[170] J. C. Slater, "Atomic shielding constants," Physical Review, vol. 36, p. 57, 1930.

[171] S. F. Boys, "Electronic wave functions. i. a general method of calculation for the stationary states of any molecular system," Proceedings of the Royal Society of London. Series A. Mathematical and Physical Sciences, vol. 200, p. 542, 1950.

[172] A. Jalbout, F. Nazari e L. Turker, "Gaussian-based computations in molecular science," Journal of Molecular Structure: THEOCHEM, vol. 671, p. 1, 2004.

[173] C. Fiolhais, F. Nogueira e M. A. L. Marques, eds., A Primer in Density Functional Theory, vol. 620 of Lecture Notes in Physics. Springer Berlin Heidelberg, 2003.

[174] D. Pines e P. Nozieres, Theory of Quantum Liquids. Benjamin, 1966. 
[175] G. Giuliani e G. Vignale, Quantum Theory of the Electron Liquid. Cambridge University Press, 2005.

[176] E. K. U. Gross e W. Kohn, "Local density-functional theory of frequencydependent linear response," Physical Review Letters, vol. 55, p. 2850, 1985.

[177] L. Landau e E. Lifshitz, Mechanics, vol. 1 of A Course of Theoretical Physics. Pergamon Press, 1976.

[178] M. E. Casida, "Time-Dependent Density Functional Response Theory for Molecules," in Recent Advances in Density Functional Methods (D. E. Chong, ed.), p. 155, World Scientific, 1995.

[179] W. Hieringer e A. Görling, "Failure of time-dependent density functional methods for excitations in spatially separated systems," Chemical Physics Letters, vol. 419, p. 557, 2006.

[180] J. Autschbach, "Charge-Transfer Excitations and Time-Dependent Density Functional Theory: Problems and Some Proposed Solutions," ChemPhysChem, vol. 10, p. 1757, 2009.

[181] Y. Zhao e D. G. Truhlar, "A new local density functional for main-group thermochemistry, transition metal bonding, thermochemical kinetics, and noncovalent interactions," Journal of Chemical Physics, vol. 125, p. 194101, 2006.

[182] Y. Zhao e D. G. Truhlar, "Density Functional for Spectroscopy: No Long-Range Self-Interaction Error, Good Performance for Rydberg and Charge-Transfer States, and Better Performance on Average than B3LYP for Ground States," Journal of Physical Chemistry A, vol. 110, p. 13126, 2006.

[183] Y. Tawada, T. Tsuneda, S. Yanagisawa, T. Yanai e K. Hirao, "A long-rangecorrected time-dependent density functional theory.," Journal of Chemical Physics, vol. 120, p. 8425, 2004.

[184] L. Kronik, T. Stein, S. Refaely-Abramson e R. Baer, "Excitation Gaps of FiniteSized Systems from Optimally Tuned Range-Separated Hybrid Functionals," Journal of Chemical Theory and Computation, vol. 8, p. 1515, 2012. 
[185] J. D. Chai e M. Head-Gordon, "Systematic optimization of long-range corrected hybrid density functionals," Journal of Chemical Physics, vol. 128, p. 1, 2008.

[186] J.-D. Chai e M. Head-Gordon, "Optimal operators for Hartree-Fock exchange from long-range corrected hybrid density functionals," Chemical Physics Letters, vol. 467 , p. 176, 2008.

[187] D. Marx e J. Hutter, "Ab initio molecular dynamics: Theory and implementation," in NIC Series Volume 3: Modern methods and algorithms of Quantum Chemistry (J. Grotendorst, ed.), p. 301, John von Neumann Institute for Computing, 2000.

[188] J.-P. Hansen e I. R. McDonald, Theory of simple liquids. Academic Press, 3 ed., 2006.

[189] N. Morgon e K. Coutinho, Métodos de química teórica e modelagem molecular. Editora Livraria da Física, 2007.

[190] J. VandeVondele, M. Krack, F. Mohamed, M. Parrinello, T. Chassaing e J. Hutter, "QUICKSTEP: Fast and accurate density functional calculations using a mixed gaussian and plane waves approach," Computer Physics Communications, vol. 167, p. 103, 2005.

[191] J. Hutter, M. Iannuzzi, F. Schiffmann e J. VandeVondele, "CP2K: atomistic simulations of condensed matter systems," Wiley Interdisciplinary ReviewsComputational Molecular Science, vol. 4, p. 15, 2014.

[192] G. Lippert, M. Parrinello e J. Hutter, "A hybrid gaussian and plane wave density functional scheme," Molecular Physics, vol. 92, p. 477, 1997.

[193] G. B. Bachelet, D. R. Hamann e M. Schlüter, "Pseudopotentials that work: From h to pu," Physical Review B, vol. 26, p. 4199, 1982.

[194] D. R. Hamann, M. Schlüter e C. Chiang, "Norm-conserving pseudopotentials," Physical Review Letters, vol. 43, p. 1494, 1979.

[195] S. Goedecker, M. Teter e J. Hutter, "Separable dual-space gaussian pseudopotentials," Physical Review B, vol. 54, p. 1703, 1996. 
[196] C. Hartwigsen, S. Goedecker e J. Hutter, "Relativistic separable dual-space gaussian pseudopotentials from H to Rn," Physical Review B, vol. 58, p. 3641, 1998.

[197] W. L. Jorgensen, D. S. Maxwell e J. TiradoRives, "Development and testing of the OPLS all-atom force field on conformational energetics and properties of organic liquids," Journal of the American Chemical Society, vol. 118, p. 11225, 1996.

[198] D. A. Case, T. E. Cheatham, T. Darden, H. Gohlke, R. Luo, K. M. Merz, A. Onufriev, C. Simmerling, B. Wang e R. J. Woods, "The AMBER biomolecular simulation programs," Journal of Computational Chemistry, vol. 26, p. 1668, 2005 .

[199] P. K. Weiner e P. A. Kollman, "AMBER - assisted model-building with energy refinement - A general program for modeling molecules and their interactions," Journal of Computational Chemistry, vol. 2, p. 287, 1981.

[200] D. P. Geerke e W. F. van Gunsteren, "Force field evaluation for biomolecular simulation: Free enthalpies of solvation of polar and apolar compounds in various solvents," Chemphyschem, vol. 7, p. 671, 2006.

[201] C. Oostenbrink, A. Villa, A. E. Mark e W. F. Van Gunsteren, "A biomolecular force field based on the free enthalpy of hydration and solvation: The GROMOS force-field parameter sets 53A5 and 53A6," Journal of Computational Chemistry, vol. 25, p. 1656, 2004.

[202] N. Schmid, A. P. Eichenberger, A. Choutko, S. Riniker, M. Winger, A. E. Mark e W. F. van Gunsteren, "Definition and testing of the GROMOS force-field versions 54A7 and 54B7," European Biophysics Journal with Biophysics Letters, vol. 40, p. $843,2011$.

[203] W. R. P. Scott, P. H. Hunenberger, I. G. Tironi, A. E. Mark, S. R. Billeter, J. Fennen, A. E. Torda, T. Huber, P. Kruger e W. F. van Gunsteren, "The GROMOS biomolecular simulation program package," Journal of Physical Chemistry A, vol. 103, p. 3596, 1999. 
[204] B. R. Brooks, R. E. Bruccoleri, B. D. Olafson, D. J. States, S. Swaminathan e M. Karplus, "CHARMM - A program for macromolecular energy, minimization, and dynamics calculations," Journal of Computational Chemistry, vol. 4, p. 187, 1983.

[205] B. R. Brooks, I. Brooks, C. L., J. Mackerell, A. D., L. Nilsson, R. J. Petrella, B. Roux, Y. Won, G. Archontis, C. Bartels, S. Boresch, A. Caflisch, L. Caves, Q. Cui, A. R. Dinner, M. Feig, S. Fischer, J. Gao, M. Hodoscek, W. Im, K. Kuczera, T. Lazaridis, J. Ma, V. Ovchinnikov, E. Paci, R. W. Pastor, C. B. Post, J. Z. Pu, M. Schaefer, B. Tidor, R. M. Venable, H. L. Woodcock, X. Wu, W. Yang, D. M. York e M. Karplus, "CHARMM: The biomolecular simulation program," Journal of Computational Chemistry, vol. 30, p. 1545, 2009.

[206] S. Patel e C. L. Brooks, "CHARMM fluctuating charge force field for proteins: I. Parameterization and application to bulk organic liquid simulations," Journal of Computational Chemistry, vol. 25, p. 1, 2004.

[207] W. L. Jorgensen e T. B. Nguyen, "Monte-carlo simulations of the hydration of substituted benzenes with OPLS potential functions," Journal of Computational Chemistry, vol. 14, p. 195, 1993.

[208] W. L. Jorgensen e N. A. McDonald, "Development of an all-atom force field for heterocycles. properties of liquid pyridine and diazenes," Theochem-Journal of Molecular Structure, vol. 424, p. 145, 1998.

[209] M. L. P. Price, D. Ostrovsky e W. L. Jorgensen, "Gas-phase and liquid-state properties of esters, nitriles, and nitro compounds with the OPLS-AA force field," Journal of Computational Chemistry, vol. 22, p. 1340, 2001.

[210] W. L. Jorgensen, J. P. Ulmschneider e J. Tirado-Rives, "Free energies of hydration from a generalized Born model and an all-atom force field," Journal of Physical Chemistry B, vol. 108, p. 16264, 2004.

[211] C. M. Breneman e K. B. Wiberg, "Determining atom-centered monopoles from molecular electrostatic potentials - the need for high sampling density in formamide conformational-analysis," Journal of Computational Chemistry, vol. 11, p. 361, 1990. 
[212] J. Barker e R. Watts, "Monte Carlo studies of the dielectric properties of waterlike models," Molecular Physics, vol. 26, p. 789, 1973.

[213] I. G. Tironi, R. Sperb, P. E. Smith e W. F. van Gunsteren, "A generalized reaction field method for molecular dynamics simulations," Journal of Chemical Physics, vol. 102, p. 5451, 1995.

[214] T. Darden, D. York e L. Pedersen, "Particle mesh Ewald: An N·log(N) method for Ewald sums in large systems," Journal of Chemical Physics, vol. 98, p. 10089, 1993.

[215] U. Essmann, L. Perera, M. L. Berkowitz, T. Darden, H. Lee e L. G. Pedersen, "A smooth particle mesh Ewald method," Journal of Chemical Physics, vol. 103, p. $8577,1995$.

[216] C. Sagui e T. A. Darden, "Molecular dynamics simulations of biomolecules: longrange electrostatic effects.," Annual review of biophysics and biomolecular structure, vol. 28, p. 155, 1999.

[217] E. Lindahl, B. Hess e D. van der Spoel, "GROMACS 3.0: A package for molecular simulation and trajectory analysis," Journal of Molecular Modeling, vol. 7, p. 306, 2001.

[218] D. Van der Spoel, E. Lindahl, B. Hess, G. Groenhof, A. E. Mark e H. J. C. Berendsen, "Gromacs: Fast, flexible, and free," Journal of Computational Chemistry, vol. 26, p. 1701, 2005.

[219] B. Hess, C. Kutzner, D. van der Spoel e E. Lindahl, "GROMACS 4: Algorithms for highly efficient, load-balanced, and scalable molecular simulation," Journal of Chemical Theory and Computation, vol. 4, p. 435, 2008.

[220] D. Van Der Spoel, E. Lindahl, B. Hess, A. Van Buuren, E. Apol, P. Meulenhoff, D. Tieleman, A. Sijbers, K. Feenstra e R. Van Drunen, "GROMACS user manual," 2010.

[221] D. van der Spoel e B. Hess, "GROMACS - The road ahead," Wiley Interdisciplinary Reviews-Computational Molecular Science, vol. 1, p. 710, 2011. 
[222] D. van der Spoel, P. J. van Maaren e C. Caleman, "GROMACS molecule \& liquid database," Bioinformatics, vol. 28, p. 752, 2012.

[223] R. W. Hockney, S. P. Goel e J. W. Eastwood, "Quiet high-resolution computer models of a plasma," Journal of Computational Physics, vol. 14, p. 148, 1974.

[224] G. Bussi, D. Donadio e M. Parrinello, "Canonical sampling through velocity rescaling," Journal of Chemical Physics, vol. 126, 2007.

[225] G. Bussi e M. Parrinello, "Stochastic thermostats: comparison of local and global schemes," Computer Physics Communications, vol. 179, p. 26, 2008.

[226] H. C. Georg, K. Coutinho e S. Canuto, "Solvent effects on the UV-visible absorption spectrum of benzophenone in water: A combined Monte Carlo quantum mechanics study including solute polarization," Journal of Chemical Physics, vol. 126, 2007.

[227] S. Canuto, K. Coutinho e D. Trzesniak, "New developments in Monte Carlo/quantum mechanics methodology. The solvatochromism of $\beta$-carotene in different solvents," in Advances in Quantum Chemistry, Vol 41: a Tribute to the Life and Work of Per-Olov Löwdin (J. R. Sabin e E. Brandas, eds.), Advances in Quantum Chemistry, p. 161, Academic Press, 2002.

[228] K. Coutinho e S. Canuto, "DICE: A Monte Carlo program for molecular liquid simulation, v. 2.9." University of São Paulo, São Paulo, 2003.

[229] X. Daura, K. Gademann, B. Jaun, D. Seebach, W. F. van Gunsteren e A. E. Mark, "Peptide Folding: When Simulation Meets Experiment," Angewandte Chemie International Edition, vol. 38, p. 236, 1999.

[230] P. Atkins e R. Friedman, Molecular Quantum Mechanics. OUP Oxford, 2011.

[231] M. J. Frisch, G. W. Trucks, H. B. Schlegel, G. E. Scuseria, M. A. Robb, J. R. Cheeseman, G. Scalmani, V. Barone, B. Mennucci, G. A. Petersson, H. Nakatsuji, M. Caricato, X. Li, H. P. Hratchian, A. F. Izmaylov, J. Bloino, G. Zheng, J. L. Sonnenberg, M. Hada, M. Ehara, K. Toyota, R. Fukuda, J. Hasegawa, M. Ishida, T. Nakajima, Y. Honda, O. Kitao, H. Nakai, T. Vreven, J. A. Montgomery, Jr., J. E. Peralta, F. Ogliaro, M. Bearpark, J. J. Heyd, E. Brothers, 
K. N. Kudin, V. N. Staroverov, R. Kobayashi, J. Normand, K. Raghavachari, A. Rendell, J. C. Burant, S. S. Iyengar, J. Tomasi, M. Cossi, N. Rega, J. M. Millam, M. Klene, J. E. Knox, J. B. Cross, V. Bakken, C. Adamo, J. Jaramillo, R. Gomperts, R. E. Stratmann, O. Yazyev, A. J. Austin, R. Cammi, C. Pomelli, J. W. Ochterski, R. L. Martin, K. Morokuma, V. G. Zakrzewski, G. A. Voth, P. Salvador, J. J. Dannenberg, S. Dapprich, A. D. Daniels, Ö. Farkas, J. B. Foresman, J. V. Ortiz, J. Cioslowski e D. J. Fox, "Gaussian 09 Revision A.02." Gaussian Inc. Wallingford CT 2009.

[232] K. B. Wiberg, "Basis set effects on calculated geometries: 6-311++G** vs. augcc-pVDZ," Journal of Computational Chemistry, vol. 25, p. 1342, 2004.

[233] K. A. Peterson, "Systematically convergent basis sets with relativistic pseudopotentials. I. Correlation consistent basis sets for the post-d group 13-15 elements," Journal of Chemical Physics, vol. 119, p. 11099, 2003.

[234] K. A. Peterson, D. Figgen, E. Goll, H. Stoll e M. Dolg, "Systematically convergent basis sets with relativistic pseudopotentials. II. Small-core pseudopotentials and correlation consistent basis sets for the post-d group 16-18 elements," Journal of Chemical Physics, vol. 119, p. 11113, 2003.

[235] K. A. Peterson, B. C. Shepler, D. Figgen e H. Stoll, "On the spectroscopic and thermochemical properties of $\mathrm{ClO}, \mathrm{BrO}, \mathrm{IO}$, and their anions," Journal of Physical Chemistry A, vol. 110, p. 13877, 2006.

[236] S. F. Boys e F. Bernardi, "The calculation of small molecular interactions by the differences of separate total energies. Some procedures with reduced errors," Molecular Physics, vol. 19, p. 553, 1970.

[237] S. Simon, M. Duran e J. J. Dannenberg, "How does basis set superposition error change the potential surfaces for hydrogen bonded dimers?," Journal of Chemical Physics, vol. 105, p. 11024, 1996.

[238] Y. Zhao e D. G. Truhlar, "The M06 suite of density functionals for main group thermochemistry, thermochemical kinetics, noncovalent interactions, excited states, and transition elements: two new functionals and systematic testing of four 
M06-class functionals and 12 other functionals," Theoretical Chemistry Accounts, vol. 120, p. 215, 2008.

[239] D. A. Binder e M. M. Kreevoy, "Speciation of 1-alkyl-4-cyanopyridinium iodide," Journal of Physical Chemistry A, vol. 101, p. 1774, 1997.

[240] A. E. Reed, R. B. Weinstock e F. Weinhold, "Natural population analysis," Journal of Chemical Physics, vol. 83, p. 735, 1985.

[241] F. Martin e H. Zipse, "Charge distribution in the water molecule - A comparison of methods," Journal of computational chemistry, vol. 26, p. 97, 2005.

[242] M. Kallay e J. Gauss, "Calculation of excited-state properties using general coupled-cluster and configuration-interaction models," Journal of Chemical Physics, vol. 121, p. 9257, 2004.

[243] H. Koch, R. Kobayashi, A. S. Demeras e P. Jorgensen, "Calculation of sizeintensive transition moments from the coupled cluster singles and doubles linearresponse function," Journal of Chemical Physics, vol. 100, p. 4393, 1994.

[244] T. B. Pedersen e H. Koch, "Coupled cluster response functions revisited," Journal of Chemical Physics, vol. 106, p. 8059, 1997.

[245] J. F. Stanton e R. J. Bartlett, "The equation of motion coupled-cluster method A systematic biorthogonal approach to molecular-excitation energies, transitionprobabilities, and excited-state properties," Journal of Chemical Physics, vol. 98, p. 7029, 1993.

[246] K. Medda, P. Chatterjee, A. K. Chandra e S. Bagchi, "Linear solvation energy relationships: charge transfer band maxima of $\mathrm{N}$-alkylpyridinium iodides in pure solvents," Journal of the Chemical Society, Perkin Transactions 2, vol. 2, p. 343, 1992.

[247] A. Dreuw e M. Head-Gordon, "Single-Reference ab Initio Methods for the Calculation of Excited States of Large Molecules," Chemical Reviews, vol. 105, p. 4009, 2005. 
[248] R. a. Mackay, J. R. Landolph e E. J. Poziomek, "Experimental evidence concerning the nature of the two charge-transfer bands in pyridinium iodides," Journal of the American Chemical Society, vol. 93, p. 5026, 1971.

[249] R. A. Mackay e E. J. Poziomek, "Pyridinium iodide salts. Correlation of experimental parameters with self-consistent extended Hueckel (SCEH) calculations," Journal of the American Chemical Society, vol. 94, p. 4167, 1972.

[250] S. Zahn, M. Brehm, M. Brüssel, O. Hollóczki, M. Kohagen, S. Lehmann, F. Malberg, A. S. Pensado, M. Schöppke, H. Weber e B. Kirchner, "Understanding ionic liquids from theoretical methods," Journal of Molecular Liquids, vol. 192, p. 71, 2014.

[251] G. Lippert, J. Hutter e M. Parrinello, "The gaussian and augmented-plane-wave density functional method for ab initio molecular dynamics simulations," Theoretical Chemistry Accounts, vol. 103, p. 124, 1999.

[252] J. VandeVondele e J. Hutter, "Gaussian basis sets for accurate calculations on molecular systems in gas and condensed phases," Journal of Chemical Physics, vol. 127, p. 114105, 2007.

[253] B. J. C. Cabral, K. Coutinho e S. Canuto, "Born-Oppenheimer molecular dynamics and electronic properties of chlorophyll-c2 in liquid methanol," Journal of Chemical Physics, vol. 138, 2013.

[254] B. J. Cabral, K. Coutinho e S. Canuto, "Dynamics of endo- vs. exo-complexation and electronic absorption of calix[4]arene-Ar2," Chemical Physics Letters, vol. 612 , p. 266, 2014.

[255] B. J. Cabral, V. W. D. Cruzeiro, K. Coutinho e S. Canuto, "Free base phthalocyanine: Influence of thermal effects and dimerization on the electronic absorption spectrum," Chemical Physics Letters, vol. 595-596, p. 97, 2014.

[256] I. J. McNaught, "Structural parameters of methyl iodide by infrared spectroscopy," Journal of Chemical Education, vol. 59, p. 879, 1982.

[257] N. Sundaraganesan, C. Meganathan, B. Anand, B. D. Joshua e C. Lapouge, "Vibrational spectra and assignments of 2-amino-5-iodopyridine by ab initio 
Hartree-Fock and density functional methods," Spectrochimica Acta - Part A: Molecular and Biomolecular Spectroscopy, vol. 67, p. 830, 2007.

[258] H. Bekker, H. J. C. Berendsen, E. J. Dijkstra, S. Achterop, R. Vondrumen, D. Vanderspoel, A. Sijbers, H. Keegstra, B. Reitsma e M. K. R. Renardus, "GROMACS - A parallel computer for molecular-dynamics simulations," Physics Computing 92, p. 252, 1993.

[259] H. J. C. Berendsen, D. Vanderspoel e R. Vandrunen, "GROMACS - A messagepassing parallel molecular-dynamics implementation," Computer Physics Communications, vol. 91, p. 43, 1995.

[260] M. M. Reif e P. H. Hünenberger, "Computation of methodology-independent single-ion solvation properties from molecular simulations. IV. Optimized Lennard-Jones interaction parameter sets for the alkali and halide ions in water," Journal of Chemical Physics, vol. 134, p. 144104, 2011.

[261] M. Thomas, M. Brehm, R. Fligg, P. Vöhringer e B. Kirchner, "Computing vibrational spectra from ab initio molecular dynamics," Physical Chemistry Chemical Physics, vol. 15, p. 6608, 2013.

[262] C. Cramer, Essentials of Computational Chemistry: Theories and Models. Wiley, 2005.

[263] H. Böhm, I. McDonald e P. Madden, "An effective pair potential for liquid acetonitrile," Molecular Physics, vol. 49, p. 347, 1983.

[264] W. L. Jorgensen e J. M. Briggs, "Monte Carlo simulations of liquid acetonitrile with a three-site model," Molecular Physics, vol. 63, p. 547, 1988.

[265] E. Guàrdia, R. Pinzón, J. Casulleras, M. Orozco e F. J. Luque, "Comparison of Different Three-site Interaction Potentials for Liquid Acetonitrile," Molecular Simulation, vol. 26, p. 287, 2001.

[266] P. J. Gee e W. F. van Gunsteren, "Acetonitrile revisited: a molecular dynamics study of the liquid phase," Molecular Physics, vol. 104, p. 477, 2006. 
[267] H. J. C. Berendsen, J. P. M. Postma, W. F. van Gunsteren, A. DiNola e J. R. Haak, "Molecular dynamics with coupling to an external bath," Journal of Chemical Physics, vol. 81, p. 3684, 1984. 


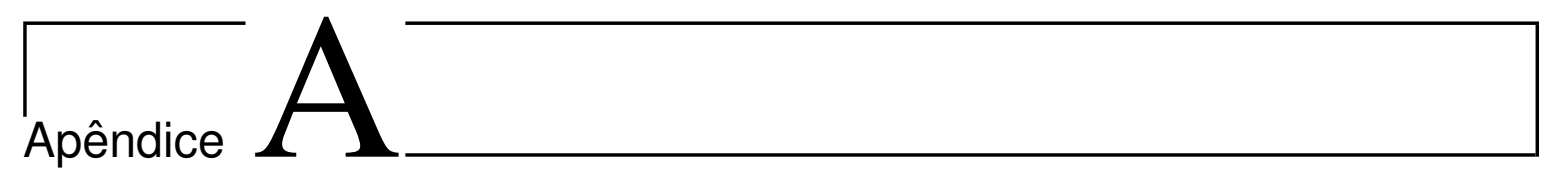

\section{Informação suplementar}

Neste capítulo são apresentados vários resultados de cálculos realizados nos CTC estudados neste trabalho que, com o objetivo de manter o texto principal mais conciso, foram colocados aqui. 


\section{A.1 Geometrias otimizadas}

Arquivo "XYZ" com as coordenadas cartesianas do complexo $C_{4}(4 C P)^{+} I^{-}$. Funcional B3LYP. Bases 6-311+G(d,p) para C, N, H e aug-cc-PVDZ-PP para I, Br.

26

C44CPI opt B3LYP/6-311+G(d,p)/aug-cc-pVDZ-pp

$\begin{array}{llll}\mathrm{N} & -2.927831 & -4.283201 & 0.035554\end{array}$

$\begin{array}{llll}\text { C } & -1.912554 & -3.740086 & 0.131801\end{array}$

C $\quad-0.666692 \quad-3.051187 \quad 0.231494$

$\begin{array}{llll}\text { C } & -0.619314 & -1.635323 & 0.067872\end{array}$

$\begin{array}{llll}\text { C } & 0.589872 & -1.009535 & 0.097602\end{array}$

N $\quad 1.753122 \quad-1.710822 \quad 0.272093$

$\begin{array}{llll}\text { C } & 1.717809 & -3.034206 & 0.578648\end{array}$

$\begin{array}{llll}\text { C } & 0.502054 & -3.734388 & 0.499324\end{array}$

$\begin{array}{llll}\mathrm{H} & -1.522298 & -1.064443 & -0.094447\end{array}$

$\begin{array}{llll}\mathrm{H} & 0.695872 & 0.056646 & -0.043107\end{array}$

$\begin{array}{llll}\mathrm{H} & 2.661686 & -3.548808 & 0.627805\end{array}$

$\begin{array}{llll}\mathrm{H} & 0.509603 & -4.800666 & 0.674659\end{array}$

$\begin{array}{llll}\text { C } & 3.043639 & -0.981542 & 0.286099\end{array}$

$\begin{array}{llll}\mathrm{H} & 3.828802 & -1.737980 & 0.272722\end{array}$

$\begin{array}{llll}\mathrm{H} & 3.110819 & -0.415729 & -0.647579\end{array}$

$\begin{array}{llll}\text { C } & 3.204733 & -0.067478 & 1.504827\end{array}$

$\begin{array}{llll}\mathrm{H} & 3.031023 & -0.656357 & 2.410007\end{array}$

$\begin{array}{llll}\mathrm{H} & 2.441912 & 0.719380 & 1.478516\end{array}$

$\begin{array}{llll}\text { C } & 4.597810 & 0.572041 & 1.544409\end{array}$

$\begin{array}{llll}\mathrm{H} & 5.354015 & -0.219873 & 1.593183\end{array}$

$\begin{array}{llll}\mathrm{H} & 4.782623 & 1.120264 & 0.611881\end{array}$

$\begin{array}{llll}\text { C } & 4.774774 & 1.516437 & 2.736739\end{array}$

$\begin{array}{llll}\mathrm{H} & 4.628332 & 0.986846 & 3.681927\end{array}$

$\begin{array}{llll}\mathrm{H} & 4.055281 & 2.340153 & 2.700579\end{array}$

$\begin{array}{llll}\mathrm{H} & 5.777402 & 1.951135 & 2.748013\end{array}$

I $\quad 2.089508 \quad-3.411276 \quad 3.459380$ 
Arquivo "XYZ" da geometria otimizada do complexo $\mathrm{C}_{4}(4 C P)^{+} \mathrm{Br}^{-}$.

26

C44CPBr opt B3LYP/6-311+G(d,p)/aug-cc-pVDZ-pp

$-2.925802$

$-4.289884$

0.024329

C

$-1.911170$

$-3.748300$

0.131404

C

$-0.664410-3.059251$

0.245320

C

$-0.618242-1.642126$

0.077228

C

$0.591922-1.020910$

0.111011

N

$1.752932-1.721669$

0.298416

C

$1.717880-3.041455$

0.635940

C

0.496169

$-3.744257$

0.517545

$\mathrm{H}$

$-1.519663$

$-1.072474$

$-0.096103$

$\mathrm{H}$

0.701273

0.043748

$-0.039915$

$\mathrm{H}$

2.659235

$-3.562575$

0.626581

$\mathrm{H}$

0.500277

$-4.811633$

0.685212

C

3.042367

$-0.992795$

0.308339

$\mathrm{H}$

3.828271

$-1.748581$

0.292065

$\mathrm{H}$

3. 106251

$-0.426956$

$-0.625721$

C

3.211382

$-0.076314$

1.524453

$\mathrm{H}$

3.049203

$-0.666848$

2.429969

$\mathrm{H}$

2.442827

0.705232

1.505030

4.599753

0.573949

1.547851

$\mathrm{H}$

5.363201

$-0.211506$

1.589672

$\mathrm{H}$

4.770389

1.121693

0.612319

4.782943

1.523052

2.735573

$\mathrm{H}$

4.650116

0.995531

3.684039

$\mathrm{H}$

4.056967

2. 341295

2.704756

$\mathrm{H}$

5.782411

1. 965270

2.735341

$\mathrm{Br}$

2.035518

$-3.272233$

3. 199346 
Arquivo "XYZ" para a estrutura I do complexo formado com o $C_{3}$ bis $(4 C P)^{2+}$.

\begin{tabular}{lrrr} 
34 & & & \\
Energy: & -686968.5701199 & & \\
N & -7.39989 & -0.86331 & -0.31161 \\
C & -6.31530 & -0.46901 & -0.30501 \\
C & -4.97532 & 0.02517 & -0.29619 \\
C & -3.91284 & -0.79430 & 0.09869 \\
C & -4.70519 & 1.34716 & -0.68712 \\
C & -2.62698 & -0.28599 & 0.10859 \\
C & -3.40511 & 1.79961 & -0.66451 \\
N & -2.38971 & 0.99355 & -0.26842 \\
C & -0.98678 & 1.50442 & -0.25695 \\
C & -0.46810 & 1.69424 & 1.17287 \\
C & 0.89165 & 2.40378 & 1.25594 \\
N & 2.03540 & 1.67964 & 0.64270 \\
C & 2.61426 & 2.16203 & -0.49972 \\
C & 2.48899 & 0.53280 & 1.20524 \\
C & 3.72076 & 1.56789 & -1.03853 \\
C & 3.64250 & -0.08249 & 0.72296 \\
C & 4.28034 & 0.43713 & -0.39426 \\
C & 5.46982 & -0.16352 & -0.90363 \\
N & 6.43633 & -0.63739 & -1.32102 \\
H & -4.07515 & -1.82236 & 0.39477 \\
H & -5.49840 & 2.00943 & -1.00657 \\
H & -1.75256 & -0.88360 & 0.37431 \\
H & -3.14037 & 2.80518 & -0.96099 \\
H & -0.38011 & 0.75970 & -0.78247 \\
H & -0.99186 & 2.44023 & -0.81696 \\
H & -0.40760 & 0.71714 & 1.65895 \\
H & -1.16656 & 2.31071 & 1.74796 \\
H & 0.85267 & 3.38520 & 0.78162 \\
H & 1.13972 & 2.56528 & 2.30638 \\
H & 2.17226 & 3.05181 & -0.92514 \\
H & 2.00728 & 0.20464 & 2.11275 \\
H & 4.17058 & 1.97795 & -1.93209 \\
H & 4.00753 & -0.96883 & 1.22257 \\
I & 0.71720 & -1.82705 & 0.14194 \\
& & & \\
\hline
\end{tabular}


Arquivo "XYZ" para a estrutura II do complexo formado com o $C_{3}$ bis $(4 C P)^{2+}$.

$\begin{array}{lcrr}\text { 34 } & & & \\ \text { Energy : } & -686963.5789535 & & \\ \text { N } & 4.14028 & 3.55579 & -0.44954 \\ \text { C } & 3.63729 & 2.52345 & -0.56797 \\ \text { C } & 3.01255 & 1.25126 & -0.73184 \\ \text { C } & 2.43638 & 0.89779 & -1.97576 \\ \text { C } & 2.95133 & 0.34522 & 0.31860 \\ \text { C } & 1.87497 & -0.34091 & -2.12189 \\ \text { C } & 2.34558 & -0.89277 & 0.12323 \\ \text { N } & 1.85863 & -1.23837 & -1.09211 \\ \text { C } & 1.32340 & -2.61306 & -1.30103 \\ \text { C } & 0.00003 & -2.95005 & -0.59058 \\ \text { C } & -1.32328 & -2.61304 & -1.30113 \\ \text { N } & -1.85855 & -1.23838 & -1.09220 \\ \text { C } & -1.87484 & -0.34087 & -2.12193 \\ \text { C } & -2.34557 & -0.89282 & 0.12313 \\ \text { C } & -2.43631 & 0.89780 & -1.97581 \\ \text { C } & -2.95137 & 0.34514 & 0.31852 \\ \text { C } & -3.01256 & 1.25121 & -0.73189 \\ \text { C } & -3.63735 & 2.52339 & -0.56802 \\ \text { N } & -4.14038 & 3.55569 & -0.44954 \\ \text { H } & 2.45829 & 1.58244 & -2.81246 \\ \text { H } & 3.35401 & 0.58032 & 1.29390 \\ \text { H } & 1.46303 & -0.68043 & -3.06153 \\ \text { H } & 2.34172 & -1.65149 & 0.88980 \\ \text { H } & 1.25243 & -2.76487 & -2.37924 \\ \text { H } & 2.09444 & -3.29341 & -0.93616 \\ \text { H } & 0.00003 & -4.03867 & -0.48405 \\ \text { H } & -0.00000 & -2.54531 & 0.42662 \\ \text { H } & -1.25221 & -2.76479 & -2.37936 \\ \text { H } & -2.09435 & -3.29343 & -0.93637 \\ \text { H } & -1.46285 & -0.68035 & -3.06156 \\ \text { H } & -2.34173 & -1.65157 & 0.88968 \\ \text { H } & -2.45818 & 1.58249 & -2.81247 \\ \text { H } & -3.35412 & 0.58018 & 1.29380 \\ \text { I } & -0.00004 & -0.18405 & 2.15622\end{array}$


Arquivo "XYZ" para a estrutura III do complexo formado com o $C_{3}$ bis $(4 C P)^{2+}$.

\begin{tabular}{lcrr} 
34 & & & \\
Energy: & -686968.4363537 & & \\
N & 6.96743 & -1.39618 & 2.05832 \\
C & 6.00110 & -1.26698 & 1.44063 \\
C & 4.80510 & -1.11460 & 0.67510 \\
C & 3.87802 & -0.11374 & 0.98721 \\
C & 4.54335 & -1.97132 & -0.40720 \\
C & 2.72299 & 0.00386 & 0.23720 \\
C & 3.38093 & -1.80555 & -1.12607 \\
N & 2.49025 & -0.83732 & -0.80091 \\
C & 1.24934 & -0.65148 & -1.60311 \\
C & 0.00002 & -1.16186 & -0.86978 \\
C & -1.24932 & -0.65150 & -1.60310 \\
N & -2.49022 & -0.83735 & -0.80090 \\
C & -2.72291 & 0.00378 & 0.23727 \\
C & -3.38094 & -1.80553 & -1.12611 \\
C & -3.87795 & -0.11382 & 0.98728 \\
C & -4.54338 & -1.97128 & -0.40725 \\
C & -4.80507 & -1.11462 & 0.67511 \\
C & -6.00108 & -1.26699 & 1.44064 \\
N & -6.96741 & -1.39620 & 2.05832 \\
H & 4.04881 & 0.57594 & 1.80336 \\
H & 5.23961 & -2.75113 & -0.68442 \\
H & 1.96848 & 0.77796 & 0.41019 \\
H & 3.13242 & -2.42899 & -1.97394 \\
H & 1.14763 & 0.42748 & -1.76672 \\
H & 1.40450 & -1.15614 & -2.55699 \\
H & 0.00002 & -0.74223 & 0.13885 \\
H & 0.00003 & -2.25365 & -0.79427 \\
H & -1.14763 & 0.42746 & -1.76671 \\
H & -1.40447 & -1.15618 & -2.55698 \\
H & -1.96839 & 0.77784 & 0.41029 \\
H & -3.13246 & -2.42892 & -1.97401 \\
H & -4.04871 & 0.57582 & 1.80346 \\
H & -5.23966 & -2.75104 & -0.68451 \\
I & -0.00003 & 2.51670 & -0.08752 \\
& & & \\
\hline
\end{tabular}


Arquivo "XYZ" para a estrutura IV do complexo formado com o $C_{3} b i s(4 C P)^{2+}$.

\begin{tabular}{|c|c|c|c|}
\hline Energy: & -872774.8372749 & & \\
\hline $\mathrm{N}$ & -6.20613 & 0.81222 & -2.19968 \\
\hline $\mathrm{C}$ & -5.27872 & 0.83003 & -1.51214 \\
\hline $\mathrm{C}$ & -4.13251 & 0.86742 & -0.65941 \\
\hline C & -3.16673 & 1.88424 & -0.81200 \\
\hline C & -3.94928 & -0.10326 & 0.32205 \\
\hline $\mathrm{C}$ & -2.08992 & 1.92158 & 0.03861 \\
\hline C & -2.83905 & -0.02826 & 1.15089 \\
\hline N & -1.95611 & 0.98936 & 1.02487 \\
\hline C & -0.78248 & 1.07582 & 1.94056 \\
\hline C & 0.29778 & 0.02651 & 1.64767 \\
\hline $\mathrm{C}$ & 0.65176 & -0.02322 & 0.15719 \\
\hline N & 1.96083 & -0.68780 & -0.06885 \\
\hline C & 2.00835 & -2.02915 & -0.24061 \\
\hline C & 3.07873 & 0.07599 & -0.10502 \\
\hline $\mathrm{C}$ & 3.21716 & -2.66045 & -0.45549 \\
\hline C & 4.31145 & -0.50798 & -0.32178 \\
\hline $\mathrm{C}$ & 4.39140 & -1.89586 & -0.49484 \\
\hline C & 5.65741 & -2.52639 & -0.70967 \\
\hline N & 6.67864 & -3.03585 & -0.88228 \\
\hline $\mathrm{H}$ & -3.26145 & 2.63697 & -1.58238 \\
\hline $\mathrm{H}$ & -4.63833 & -0.92664 & 0.44393 \\
\hline $\mathrm{H}$ & -1.29780 & 2.66568 & -0.03045 \\
\hline $\mathrm{H}$ & -2.66484 & -0.74219 & 1.94079 \\
\hline $\mathrm{H}$ & -1.15548 & 0.96525 & 2.95964 \\
\hline $\mathrm{H}$ & -0.36332 & 2.07577 & 1.81689 \\
\hline $\mathrm{H}$ & -0.01388 & -0.96718 & 1.97996 \\
\hline $\mathrm{H}$ & 1.17051 & 0.33161 & 2.23282 \\
\hline $\mathrm{H}$ & -0.09418 & -0.60469 & -0.38782 \\
\hline $\mathrm{H}$ & 0.74878 & 0.98820 & -0.25176 \\
\hline $\mathrm{H}$ & 1.05206 & -2.55215 & -0.19382 \\
\hline $\mathrm{H}$ & 2.93069 & 1.15041 & 0.02074 \\
\hline H & 3.24007 & -3.73326 & -0.59010 \\
\hline H & 5.19483 & 0.11495 & -0.35817 \\
\hline I & -1.70116 & -3.01220 & 0.17646 \\
\hline & 1.46643 & 3.58974 & -0.04052 \\
\hline
\end{tabular}


Arquivo "XYZ" para a estrutura $\mathbf{V}$ do complexo formado com o $C_{3}$ bis $(4 C P)^{2+}$.

$\begin{array}{lrrr}\text { 35 } & & & \\ \text { Energy }: & -872741.3290035 & & \\ \text { N } & -6.65364 & -0.00124 & 2.82984 \\ \text { C } & -5.73774 & -0.00109 & 2.12748 \\ \text { C } & -4.60337 & -0.00083 & 1.25574 \\ \text { C } & -4.04038 & -1.21081 & 0.82772 \\ \text { C } & -4.04054 & 1.20940 & 0.82821 \\ \text { C } & -2.95337 & -1.18442 & -0.02350 \\ \text { C } & -2.95354 & 1.18351 & -0.02302 \\ \text { N } & -2.44801 & -0.00034 & -0.44137 \\ \text { C } & -1.24068 & -0.00005 & -1.30848 \\ \text { C } & -0.00006 & 0.00005 & -0.42366 \\ \text { C } & 1.24056 & 0.00046 & -1.30849 \\ \text { N } & 2.44791 & 0.00044 & -0.44141 \\ \text { C } & 2.95353 & -1.18354 & -0.02357 \\ \text { C } & 2.95320 & 1.18439 & -0.02306 \\ \text { C } & 4.04059 & -1.20971 & 0.82759 \\ \text { C } & 4.04025 & 1.21050 & 0.82811 \\ \text { C } & 4.60339 & 0.00038 & 1.25554 \\ \text { C } & 5.73775 & 0.00035 & 2.12730 \\ \text { N } & 6.65366 & 0.00032 & 2.82964 \\ \text { H } & -4.43648 & -2.16265 & 1.15419 \\ \text { H } & -4.43678 & 2.16105 & 1.15507 \\ \text { H } & -2.43057 & -2.07919 & -0.36353 \\ \text { H } & -2.43083 & 2.07849 & -0.36267 \\ \text { H } & -1.28119 & -0.89861 & -1.92550 \\ \text { H } & -1.28149 & 0.89861 & -1.92533 \\ \text { H } & 0.00012 & -0.90440 & 0.19004 \\ \text { H } & -0.00023 & 0.90429 & 0.19035 \\ \text { H } & 1.28136 & -0.89798 & -1.92565 \\ \text { H } & 1.28106 & 0.89923 & -1.92520 \\ \text { H } & 2.43083 & -2.07841 & -0.36354 \\ \text { H } & 2.43027 & 2.07926 & -0.36265 \\ \text { H } & 4.43688 & -2.16147 & 1.15406 \\ \text { H } & 4.43628 & 2.16223 & 1.15500 \\ \text { I } & 0.00071 & -3.65934 & -0.66988 \\ \text { I } & -0.00064 & 3.65960 & -0.66870\end{array}$




\section{A.2 Espectro vibracional}

Os cálculos do espectro de vibração foram realizados no mesmo nível de otimização de geometria, para o $C_{3}$ bis $(4 C P)^{2+}$ e seu complexo com iodeto, nas três estruturas de mínima energia. Os resultados são apresentados nos gráficos da Figura A.1. As estruturas sem o iodeto mostraram espectros praticamente idênticos, de forma que não há como distinguir as estruturas apenas pelo espectro vibracional. Nos complexos, entretanto, algumas mudanças no espectro permitem obter informações a respeito da interação do iodeto.

A principal mudança no espectro ocorre na intensidade dos modos de estiramento ("stretching") dos hidrogênios no plano do anel, que aumenta consideravelmente. Esses modos se localizam na região acima de $3000 \mathrm{~cm}^{-1}$ (Figura A.1) . A intensidade aumenta para $420 \mathrm{kcal} \cdot \mathrm{mol}^{-1}$ na estrutura I (interação em um anel) e para 640 kcal.mol ${ }^{-1}$ na estrutura III (interação nos dois anéis) e praticamente não aumenta na estrutura II (onde não há como ocorrer estiramento do hidrogênio na direção do iodeto).

Na região de $2300 \mathrm{~cm}^{-1}$ se localizam os estiramentos do nitrogênio do grupo nitrila. Esses modos perdem intensidade nos complexos, comparados aos piridínios sem o iodeto, onde a intensidade é alta (cerca de $200 \mathrm{kcal} . \mathrm{mol}^{-1}$ ).

Na região de $1000 \mathrm{~cm}^{-1}$ a $1700 \mathrm{~cm}^{-1}$ localizam-se grande parte dos modos vibracionais. Em $1670 \mathrm{~cm}^{-1}$ ocorrem deformações angulares ("scissoring") das ligações C-C e C-N e C-H no plano do anel. São intensas no piridínio (cerca de $300 \mathrm{~cm}^{-1}$ ) e se mantém intensas nos complexos, tanto em vácuo quanto em solução.

Em $1580 \mathrm{~cm}^{-1}$ ocorrem estiramentos assimétricos ("assimetrical stretching") nas ligações C-C e C-N do anel. Em $1480 \mathrm{~cm}^{-1}$ e $1540 \mathrm{~cm}^{-1}$ ocorrem deformações angulares na ligação C-H da cadeia carbônica e movimentos de "rocking"dos $\mathrm{H}$ do anel. Esses modos têm intensidade média ou baixa no piridínio isolado, mas ganham intensidade nos complexos.

Na região de 1200-1500 ocorrem uma série de vibrações angulares ("twisting", "wagging"e "rocking"), envolvendo hidrogênios da cadeia e do anel. Observa-se um ganho de intensidade com a interação com o iodeto. Na região de 1100-1200 cm ocorre o estiramento da ligação C-N do anel e N-C entre o nitrogênio o carbono da 


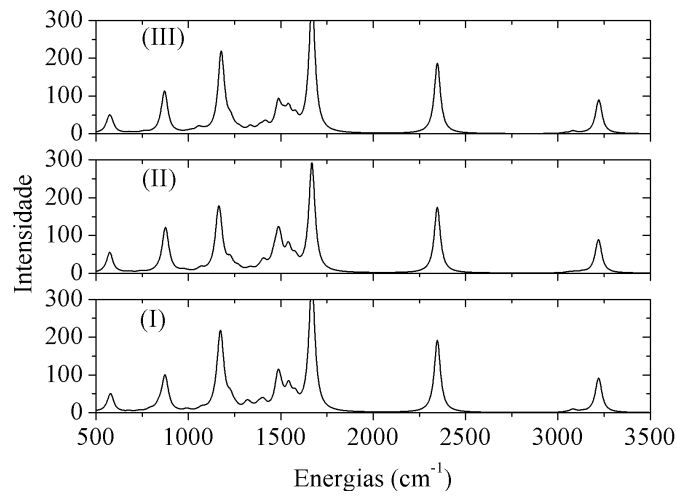

(a) $C_{3} \operatorname{bis}(4 C P)^{2+}$

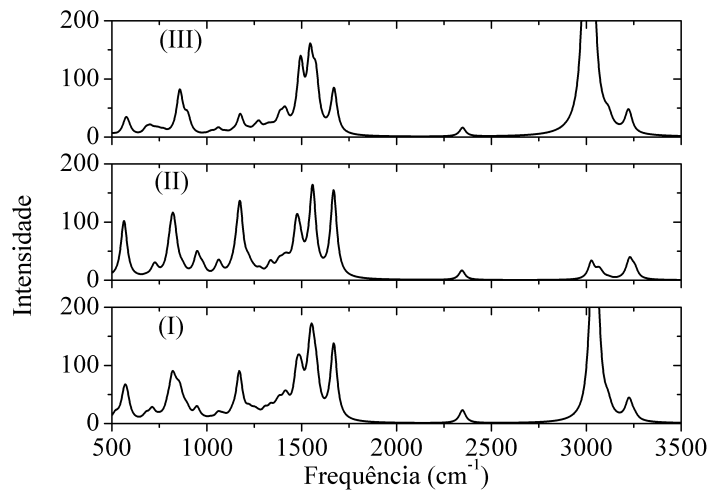

(b) $C_{3} b i s(4 C P)^{2+} I^{-}$(Vácuo)

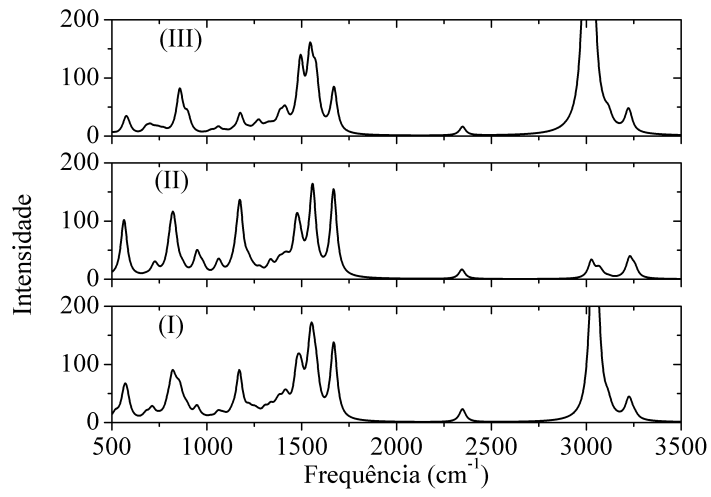

(c) $C_{3} b i s(4 C P)^{2+} I^{-}$(Acetonitrila,PCM)

Figura A.1: Convolução do espectro vibracional calculado para os complexos, intensidades em kcal.mol ${ }^{-1}$. Cálculo no nível B3LYP e bases 6-311+G(d,p) para C, H, N e aug-cc-pVDZ para $\mathbf{I}$. 
cadeia. São pouco afetadas pelo iodeto.

Em $870 \mathrm{~cm}^{-1}$ ocorrem vibrações do hidrogênio do anel para fora do plano ("wagging"). Também ganham intensidade com a interação com o iodeto.

Finalmente, na região de 500-600 $\mathrm{cm}^{-1}$ ocorrem vibrações dos átomos de $\mathrm{C} \mathrm{N}$ e $\mathrm{H}$ do anel para fora do plano. São poucos intensos quando os piridínios estão sem o iodeto, mas ganham um pouco de intensidade nos complexos.

O cálculo dos modos de vibração mostra que o espectro vibracional infravermelho não é muito eficaz na elucidação estrutural do $C_{3} b i s(4 C P)^{2+}$, mas pode ser usado como uma técnica complementar para obter informações da interação do iodeto com o piridínio. 


\section{A.3 Propriedades magnéticas}

A blindagem magnética nos átomos de $\mathrm{H}, \mathrm{C}$ e $\mathrm{N}$ foi calculada usando a metodologia GIAO e o funcional B3LYP juntamente com o conjunto de funções base 6-311 $+\mathrm{G}(\mathrm{d}, \mathrm{p})$ para tratar os átomos de H, C, N do piridínio e o pseudopotencial augcc-pVDZ-PP para o iodeto. O resultados são mostrados nos gráficos da Figura A.2.

Pelo fato do complexo ter diferentes conformações, o espectro de RMN é bem mais interessante que no caso anterior. As estruturas I e III apresentam simetria na posição dos anéis e apresentam espectros com maior degenerescência. Já a estrutura I do complexo apresenta um espectro menos degenerado e, consequentemente, com um número maior de linhas.

No caso da blindagem para o ${ }^{1} \mathrm{H}$ os menores deslocamentos ocorrem nos hidrogênios da ponte metilênica, com valor abaixo dos 4 ppm. Acima de 6 ppm localizam- se os hidrogênios do anel. Na estrutura III esses hidrogênios do anel apresentam grande degenerescência concentrando-se na região de 8 ppm. Já na estrutura I não foram observadas degenerescência, aparecendo um número bem maior de linhas no espectro. É interessante notar no espectro que, quando o iodeto interage com um hidrogênio do anel, como nas estruturas I e III, aparece uma linha acima dos 12 ppm.

$\mathrm{O}$ espectro de ${ }^{13} \mathrm{C}$ das três estruturas pode ser separado em três regiões. Abaixo de 70 ppm são os carbonos da ponte metilênica. O carbono central é o mais sensível à conformação e apresenta o menor deslocamento em relação aos outros da cadeia, com valores de $32 \mathrm{ppm}, 15 \mathrm{ppm}$ e $43 \mathrm{ppm}$ nas estruturas I, II e III, respectivamente. Os outros carbonos da cadeia não são muito sensíveis à estrutura do complexo, apresentando deslocamentos em 61 ppm (estrutura III), 62-63 ppm (estrutura I) e 67 ppm (estrutura II).

Em 120 ppm aparece uma linha com dupla degenerescência correspondendo aos dois carbonos do grupo nitrila. Esse carbono não é muito sensível à mudança conformacional ou ao efeito do solvente.

Os carbonos do anel têm deslocamentos na região de 120 a 150 ppm. Na estrutura I, o espectro é formado por várias linhas que se estendem ao longo da região de 130 ppm até 150 ppm. Já na estrutura II, o espectro concentra-se em duas regiões: 132 ppm (carbonos do lado oposto ao iodeto) e 143-145 ppm (carbonos mais próximos 
Vácuo
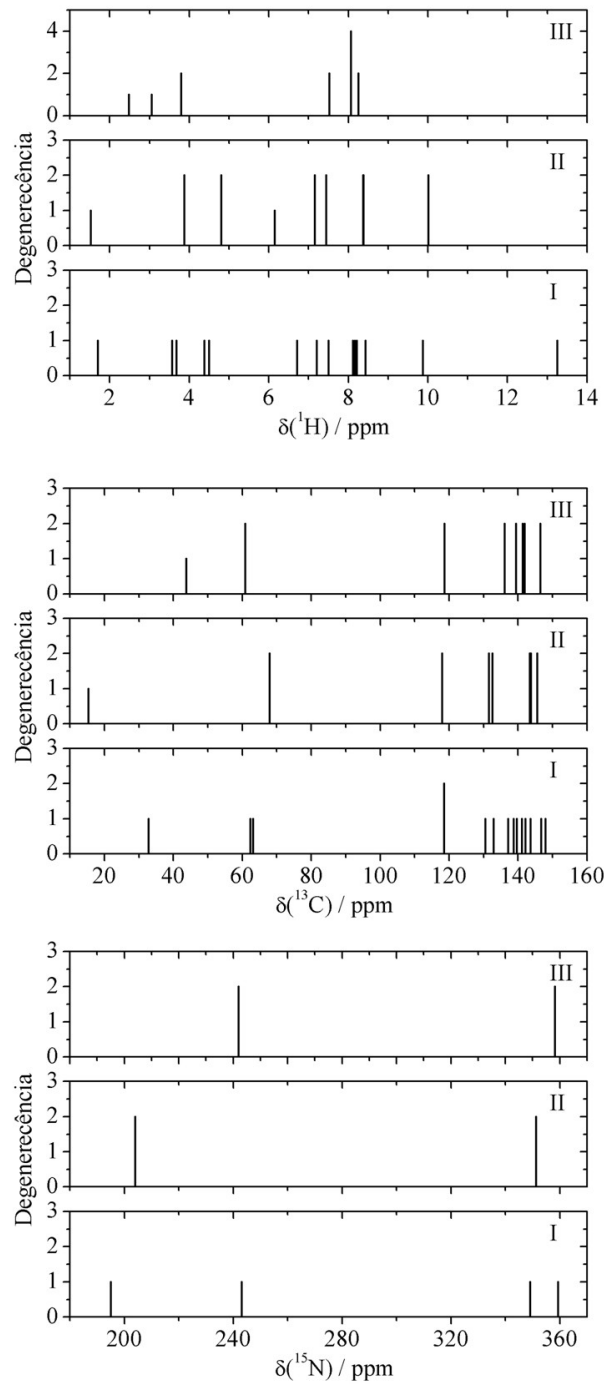

Acetonitrila (PCM)
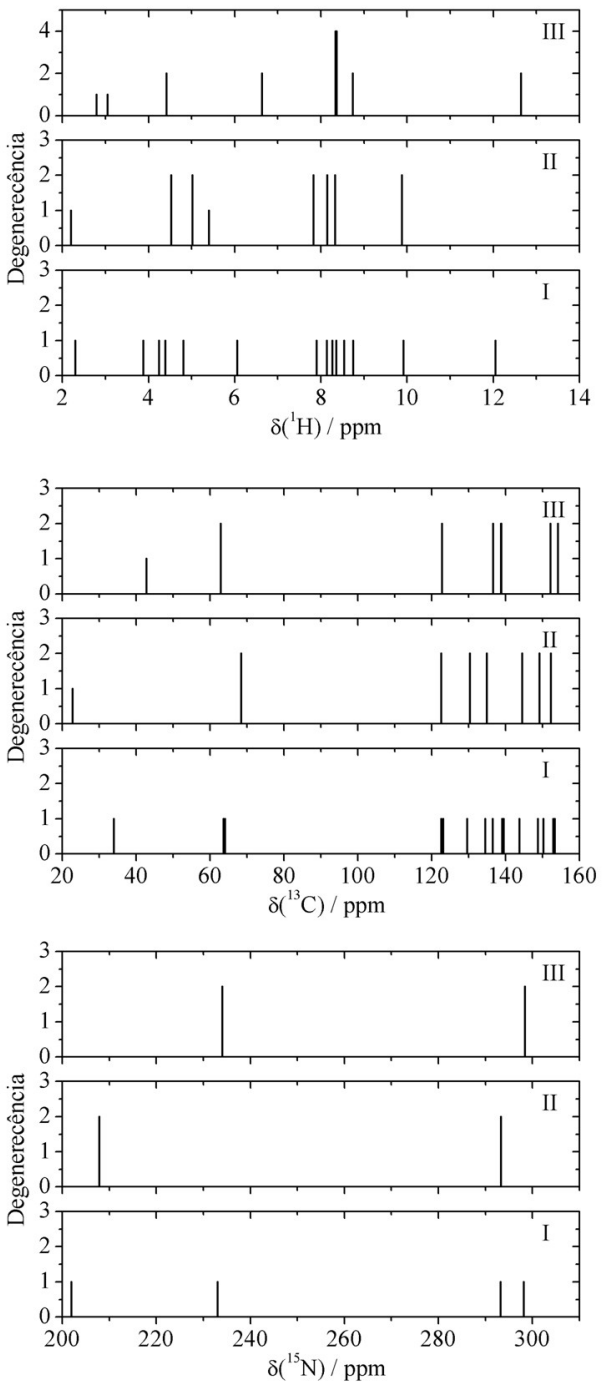

Figura A.2: Espectro de ressonância magnética nuclear, calculado a partir do deslocamento químico dos átomos de ${ }^{1} \mathrm{H},{ }^{13} \mathrm{C}$ e ${ }^{15} \mathrm{~N}$. Dois deslocamentos foram considerados degenerados quando a diferença entre eles é menor do que 0.05 ppm 
ao iodeto). A estrutura III apresenta um espectro intermediário entre os dois casos anteriores: as linhas se estendem entre 136 ppm até 146 ppm, apresentando dupla degenerescência.

Infelizmente não encontramos na literatura resultados experimentais para o espectro RMN desses complexos, mas os cálculos teóricos indicam que esse espectro pode ser uma técnica bastante útil para obter informações acerca da estrutura do $C_{3} b i s(4 C P)^{2+}$ e de sua interação com o iodeto.

Tabela A.1: Frequências vibracionais calculadas para o complexo $C_{3} b i s(4 C P)^{2+}+2 I^{-}$em Vácuo e em acetonitrila (PCM). Bases 6-311+G(d,p) para C, N, H e aug-cc-PVDZ-PP para I.

\begin{tabular}{|c|c|c|c|c|c|}
\hline \multicolumn{3}{|c|}{ Vácuo } & \multicolumn{3}{|c|}{ Acetonitrila (PCM) } \\
\hline I & II & III & I & II & III \\
\hline 17 & 15 & 16 & 9 & 21 & 17 \\
\hline 22 & 21 & 19 & 20 & 23 & 26 \\
\hline 52 & 41 & 49 & 41 & 43 & 40 \\
\hline 57 & 61 & 50 & 46 & 49 & 51 \\
\hline 85 & 86 & 93 & 61 & 72 & 59 \\
\hline 101 & 112 & 99 & 77 & 94 & 62 \\
\hline 143 & 149 & 139 & 140 & 151 & 130 \\
\hline 153 & 151 & 158 & 153 & 154 & 157 \\
\hline 202 & 209 & 178 & 209 & 223 & 179 \\
\hline 216 & 243 & 205 & 221 & 253 & 209 \\
\hline 344 & 359 & 327 & 347 & 372 & 340 \\
\hline 375 & 371 & 375 & 381 & 381 & 380 \\
\hline 397 & 400 & 400 & 403 & 403 & 406 \\
\hline 404 & 404 & 403 & 408 & 412 & 407 \\
\hline 422 & 421 & 424 & 431 & 419 & 437 \\
\hline 466 & 476 & 485 & 473 & 487 & 487 \\
\hline 562 & 563 & 563 & 571 & 572 & 573 \\
\hline 562 & 563 & 563 & 571 & 573 & 573 \\
\hline 579 & 566 & 587 & 588 & 582 & 594 \\
\hline 676 & 641 & 681 & 675 & 644 & 682 \\
\hline 682 & 681 & 703 & 683 & 683 & 704 \\
\hline 712 & 698 & 727 & 721 & 695 & 737 \\
\hline 749 & 729 & 746 & 752 & 749 & 752 \\
\hline
\end{tabular}


Tabela A.1 - Continuação

\begin{tabular}{|c|c|c|c|c|c|}
\hline \multicolumn{3}{|c|}{ Vácuo } & \multicolumn{3}{|c|}{ Acetonitrila (PCM) } \\
\hline I & II & III & I & II & III \\
\hline 793 & 802 & 772 & 801 & 813 & 770 \\
\hline 826 & 824 & 857 & 852 & 859 & 863 \\
\hline 837 & 826 & 858 & 859 & 866 & 866 \\
\hline 860 & 842 & 871 & 869 & 869 & 881 \\
\hline 882 & 872 & 899 & 880 & 883 & 884 \\
\hline 947 & 950 & 987 & 983 & 972 & 996 \\
\hline 970 & 951 & 987 & 992 & 994 & 997 \\
\hline 993 & 973 & 1028 & 1002 & 1004 & 1022 \\
\hline 1024 & 978 & 1029 & 1016 & 1005 & 1037 \\
\hline 1067 & 1060 & 1065 & 1071 & 1071 & 1073 \\
\hline 1075 & 1061 & 1066 & 1078 & 1074 & 1074 \\
\hline 1136 & 1132 & 1139 & 1144 & 1147 & 1144 \\
\hline 1166 & 1157 & 1168 & 1168 & 1162 & 1175 \\
\hline 1173 & 1175 & 1177 & 1181 & 1170 & 1185 \\
\hline 1221 & 1218 & 1218 & 1219 & 1222 & 1217 \\
\hline 1228 & 1231 & 1228 & 1225 & 1226 & 1228 \\
\hline 1245 & 1244 & 1253 & 1250 & 1252 & 1253 \\
\hline 1271 & 1280 & 1274 & 1263 & 1268 & 1274 \\
\hline 1306 & 1325 & 1308 & 1303 & 1311 & 1326 \\
\hline 1341 & 1336 & 1344 & 1343 & 1340 & 1347 \\
\hline 1368 & 1378 & 1351 & 1364 & 1369 & 1356 \\
\hline 1385 & 1396 & 1386 & 1379 & 1396 & 1386 \\
\hline 1410 & 1409 & 1410 & 1406 & 1408 & 1411 \\
\hline 1472 & 1461 & 1471 & 1482 & 1466 & 1478 \\
\hline 1477 & 1476 & 1488 & 1484 & 1481 & 1490 \\
\hline 1494 & 1492 & 1493 & 1499 & 1494 & 1497 \\
\hline 1497 & 1494 & 1498 & 1508 & 1500 & 1510 \\
\hline 1544 & 1531 & 1546 & 1549 & 1548 & 1552 \\
\hline 1556 & 1556 & 1573 & 1588 & 1589 & 1592 \\
\hline 1667 & 1666 & 1670 & 1677 & 1677 & 1679 \\
\hline 1673 & 1669 & 1671 & 1679 & 1677 & 1679 \\
\hline 2350 & 2345 & 2348 & 2351 & 2351 & 2351 \\
\hline 3020 & 3029 & 2999 & 3047 & 3041 & 3056 \\
\hline 3043 & 3072 & 3010 & 3094 & 3097 & 3090 \\
\hline
\end{tabular}


Tabela A.1 - Continuação

\begin{tabular}{llllll}
\hline \multirow{2}{*}{ Vácuo } & & \multicolumn{4}{c}{ Acetonitrila (PCM) } \\
I & II & III & I & II & III \\
\hline 3072 & 3083 & 3021 & 3100 & 3101 & 3105 \\
3112 & 3119 & 3088 & 3150 & 3153 & 3143 \\
3121 & 3216 & 3113 & 3153 & 3190 & 3144 \\
3218 & 3229 & 3217 & 3232 & 3232 & 3232 \\
3218 & 3229 & 3218 & 3233 & 3233 & 3232 \\
3229 & 3231 & 3219 & 3238 & 3236 & 3235 \\
3231 & 3255 & 3229 & 3243 & 3243 & 3242 \\
\hline
\end{tabular}


$\Gamma_{\text {Apêndice }} \mathbf{D}$

\section{Modos vibracionais via função de autocorrelação de velocidades}

A técnica de espectroscopia vibracional ou espectroscopia de infravermelho (IR, do inglês "infra-red") é uma das técnicas espectroscópicas mais usadas na caracterização molecular. A forma mais usada para o cálculo do espectro vibracional de IR é através do cálculo da segunda derivada do sistema na posição de equilíbrio na superfície de energia potencial. Ocorre em casos como configurações oriundas de dinâmica molecular, onde as moléculas não estão exatamente na posição de equilíbrio, essa técnica não pode ser usada. Ao longo dos anos, diversos métodos têm sido propostos a fim de se obter o espectro vibracional a partir da trajetória de uma dinâmica molecular. Para uma revisão desses métodos encontrada na referência [261].

Uma das formas obter um modo vibracional molecular é através da transformada de Fourier da função de autocorrelação de velocidades.

$\mathrm{Na}$ literatura encontramos duas formas distintas para a função de correlação. A forma mais conhecida relaciona diretamente a propriedade (no caso a velocidade de uma partícula) no instante $\mathrm{t}=0 \mathrm{com}$ um instante posterior $\mathrm{t}$, por meio da expressão

$$
C_{0}(t) \equiv \frac{\langle v(0) v(t)\rangle}{\langle v(0) v(0)\rangle}
$$

A outra forma de se definir a FAV é chamada de alguns autores de FAV reduzida (por 
exemplo na página 87 da segunda edição da referência [262]), e correlaciona os desvios da velocidade em relação ao valor médio, nos instantes t e $\mathrm{t}=0$,

$$
C(t)=\frac{\left\langle v_{i} v_{i+t}\right\rangle-\left\langle v_{i}\right\rangle\left\langle v_{i+t}\right\rangle}{\left\langle v^{2}\right\rangle-\langle v\rangle^{2}}
$$

Para estimar a frequência vibracional do estiramento de uma ligação $\mathrm{C}-\mathrm{H}$, por exemplo, tomamos a velocidade como sendo igual a $v(t)=\frac{d x}{d t}$, onde $x=r(C-H)$ representa a distância entre os átomos $\mathrm{C}$ e $\mathrm{H}$ no instante t. Usando qualquer uma das formas para a FAV, os modos vibracionais podem ser obtidos usando a transformada de Fourier da FAV,

$$
F(\omega)=\int e^{-i \omega t} C_{0}(t) d t
$$

Usando as definições B.1 e B.2 para a FAV, buscamos obter os modos vibracionais de para algumas ligações específicas do complexo $C_{4}(4 C P)^{+} I^{-}$. A trajetória considerada foi obtida a partir da BOMD desse complexo em vácuo, selecionando configurações igualmente espaçadas por 0.5 fs, no intervalo entre 10 ps e 80 ps, totalizando 80000 configurações.

Para avaliar a aplicação do método, usamos como exemplo o modo de estiramento da ligação tripla CN no grupo nitrila do $C_{4}(4 C P)^{+} I^{-}$. O cálculo das frequências vibracionais realizado para a geometria de mínima de energia sugere uma frequência vibracional de $2231 \mathrm{~cm}^{-1}$, usando o funcional B3LYP e bases $6-311+\mathrm{G}(\mathrm{d}, \mathrm{p})$.

O algoritmo mais eficiente para o cálculo da transformada de Fourier é o "Fast Fourier Transform"(FFT), cujo custo computacional é da forma $N \log N$ onde $N$ é o número de pontos ( $\mathrm{O}$ algoritmo direto cresce com $N^{2}$, tornando-se ineficiente para um número grande de pontos). Para a aplicação do FFT, no entanto, é necessário uma sequência discreta com um número de pontos que seja potência de 2. Portanto, não podemos usar a sequência toda, como temos uma sequência de 80000 pontos, Consideramos então, no cálculo da função $C(t)$ número de pontos iguais a $8192\left(2^{13}\right)$, $16384\left(2^{14}\right), 32768\left(2^{15}\right)$ e $65536\left(2^{16}\right)$. A Figura B.1 mostra o comportamento da Função de correlação $C(t)$ com o número crescente de pontos.

A transformada de Fourier (TF) da função $C(t)$ é mostrada na Figura B.2a. Com todos os quatros intervalos estudados, o máximo para a transformada de Fourier ocorre 

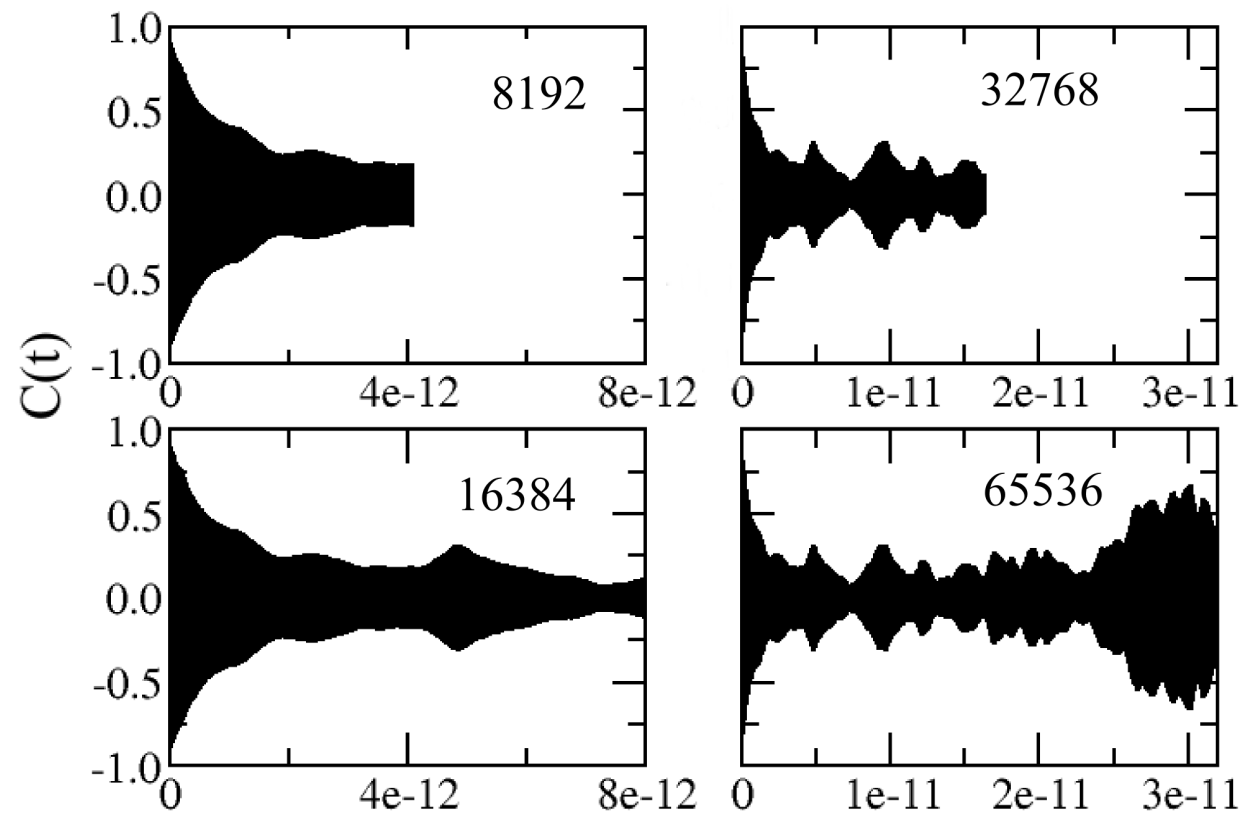

$\mathrm{t}(\mathrm{s})$

Figura B.1: Função de autocorrelação de velocidades para a ligaçao $\mathrm{C} \equiv \mathrm{N}$ do complexo $C_{4}(4 C P)^{+}$. Funcional B3LYP. Bases $6-311+\mathrm{G}(\mathrm{d}, \mathrm{p})$ para C, N, H e aug-cc-PVDZ-PP para I.

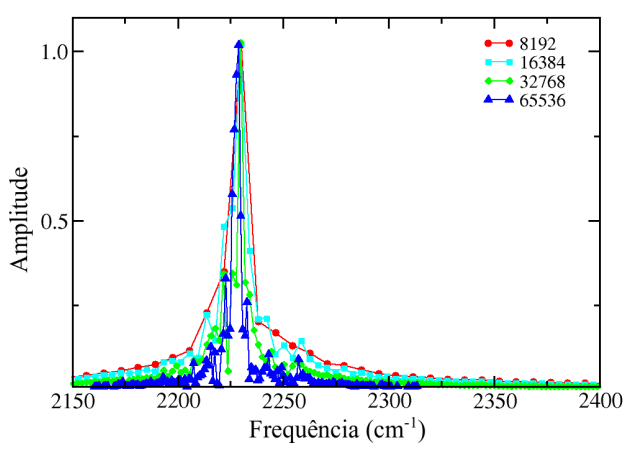

(a)

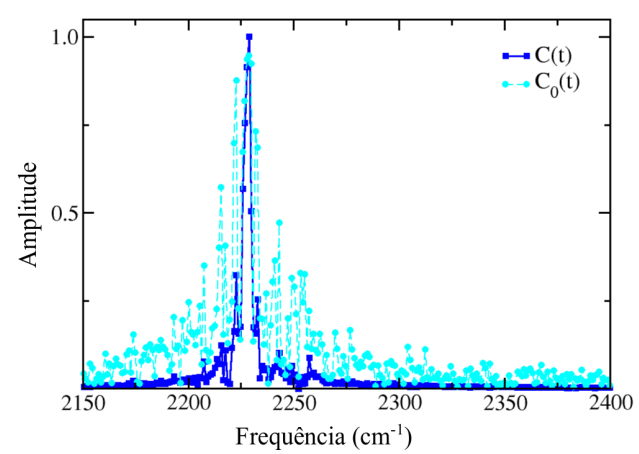

(b)

Figura B.2: A transformada de Fourier da função de autocorrelação de velocidades 
no mesmo ponto, em $2233 \mathrm{~cm}^{-1}$, em excelente acordo com o resultado calculado para a estrutura de mínima energia. O emprego de um intervalo maior de pontos no cálculo da $C(t)$ aumenta a densidade de pontos para a transformada de Fourier, fazendo com que o pico seja melhor definido. A análise da Figura B.2a mostra que um número de $2^{15}=32768$ pontos é suficiente para se obter uma boa definição do pico. As duas formas para a função de autocorrelação, $C_{0}(t)$ e $C(t)$, apresentam também o mesmo valor.

A forma reduzida, $C(t)$, resulta em uma transformada mais suave, reduzindo os ruídos, sendo assim mais adequada no emprego dos modos vibracionais, sobretudo em sistemas que apresentam vários picos ou interações mais fracas como interações não covalentes. 


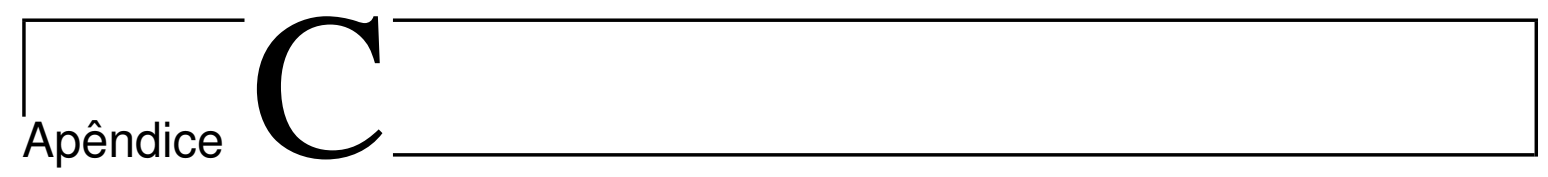

\section{Campo de força para acetonitrila}

Existem vários modelos de acetonitrila disponíveis na literatura [263-266] Aqui estudamos o modelo de acetonitrila baseado no campo de força OPLS-AA. Baseado nos parâmetros publicados em 2001 [209], criamos o arquivo de topologia para ser simulado usando o programa de dinâmica molecular GROMACS (versão 4.5.4). Para os testes, consideramos uma caixa cúbica composta por 500 moléculas de acetonitrila, e temperatura de $300 \mathrm{~K}$ e pressão de 1 bar, usando respectivamente o termostato $\mathrm{V}$ rescale [224] e barostato de Berendsen [267]. As interações eletrostática e de LennardJones foram calculadas dentro de raio de corte de $14 \mathrm{~nm}$. Todas as dinâmicas no ensemble NpT foram realizadas por um tempo total de $1 \mathrm{~ns}$, usando um tempo de integração de 1 fs.

\section{C.1 Correções de longo alcance para a interação eletrostática}

Ao todo foram realizadas quatro dinâmicas distintas. As três primeiras dinâmicas foram realizadas usando o campo de reação como correção de longo alcance para as interações além do raio de corte (com constante dielétrica igual a 35.84, referente a acetonitrila [266]). Essas correções são realizadas levando em consideração os grupos de carga definidas para a molécula no arquivo de topologia. Nesta seção estudamos como a definição dos grupos de carga pode influenciar a dinâmica do sistema. Consideramos 


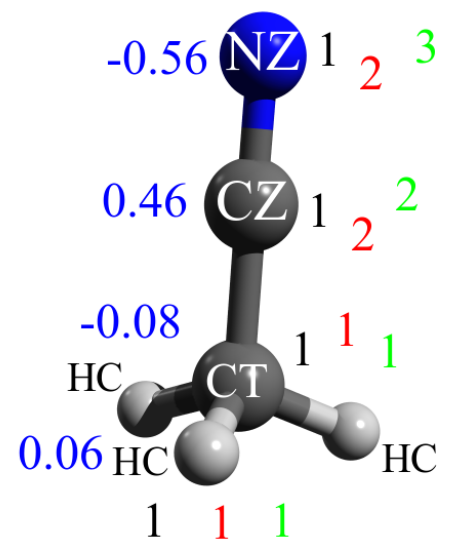

Figura C.1: Representação estrutural da molécula de acetonitrila com a definição dos grupos de carga: grupo 1 (preto), grupo 2 (vermelho), grupo 3 (verde). As cargas atômicas usadas no modelo aparecem em azul.

três grupos de cargas distintos, ilustrados na figura C.1:

- Grupo 1 (cor preta): a molécula de acetonitrila foi considera com um único grupo de carga;

- Grupo 2 (cor vermelha): definimos dois grupos, o primeiro formado pelo grupo $\mathrm{CH}_{3}$ (carga 0.1) e o segundo pelo grupo $\mathrm{C} \equiv \mathrm{N}$ (carga -0.1).

- Grupo 3 (cor verde): definimos três grupos, o primeiro formado pelo grupo $\mathrm{CH}_{3}$ (carga 0.1), o segundo pelo carbono CZ (carga 0.46) e e o terceiro pelo NZ (carga $-0.56)$.

A quarta dinâmica foi realizada usando PME ("Particle Mesh Ewald") como correção de longo alcance. Definimos um único grupo de carga para a molécula de acetonitrila. O tempo total de dinâmica foi 1ns, mas as médias foram calculadas usando os últimos 800 ps. As propriedades calculadas são mostradas na Tabela C.1.

A definição dos grupos afeta pouco os comprimentos de ligação de ângulos da molécula de acetonitrila. A variação na distância CZ-NZ no grupo 3 em relação ao grupo 1 é de apenas $0.00062 \AA$, e a variação no ângulo NZ-CT-CZ de $0.017^{\circ}$.

Em relação as dinâmicas com campo de reação, as principais grandezas que sofrem mudanças com os grupos de carga foi a densidade do sistema. Embora o grupo 1 
Tabela C.1: Propriedades calculadas a partir da dinâmica

\begin{tabular}{lccccc}
\hline \hline & \multicolumn{5}{c}{ Campo de reação } \\
& Grupo 1 & Grupo 2 & Grupo 3 & PME & OPLS-AA $^{1}$ \\
\hline r[CZ-NZ] $(\AA)$ & 1.161 & 1.161 & 1.161 & 1.160 & 1.157 \\
r[CZ-CT] $(\AA)$ & 1.459 & 1.459 & 1.459 & 1.458 & 1.458 \\
a[NZ-CZ-CT] $\left(^{\circ}\right)$ & 177 & 177 & 177 & 177 & 180 \\
En. Potencial $(\mathrm{kJ} / \mathrm{mol})$ & -5020.15 & -6176.71 & -6389.58 & -6237.65 & \\
Temperatura $(\mathrm{K})$ & 300 & 300 & 300 & 300 & \\
Pressão (bar) & $0.88 \pm 0.60$ & $1.22 \pm 0.33$ & $0.88 \pm 0.42$ & $0.98 \pm 0.06$ & \\
$\mathrm{~L}(\AA)$ & 37.919 & 35.918 & 35.667 & 35.770 & \\
$\rho\left(\mathrm{kg} / \mathrm{m}^{3}\right)$ & $625 \pm 2$ & $735 \pm 2$ & $751 \pm 1$ & $744 \pm 1$ & $765 \pm 2$ \\
\hline \hline
\end{tabular}

${ }^{1}$ Propriedades obtidas com o modelo, extraídas da ref. [209].

seja o único que possua carga nula, ele teve o maior desvio na densidade, apresentado o valor de $625 \mathrm{~kg} / \mathrm{m}^{3}$, enquanto que o resultado experimental é $777 \mathrm{~kg} / \mathrm{m}^{3}$. O grupo 3 apresenta o melhor valor, $753 \mathrm{~kg} / \mathrm{m}^{3}$, mas nesse grupo temos a pior distribuição de cargas do sistema, já que o nitrogênio NZ (-0.56) e o carbono CZ (carga 0.46) são definidos individualmente como grupos de carga. Ocorre assim um aumento na energia potencial do sistema, quando se compara o grupo 1 ao grupo 3, de -5020.15 (grupo 1) para -6389.58 (grupo 3). Comparando a função G(r) desses mesmos grupos, vemos que no grupo 3 ocorre um maior empacotamento do sistema (ver Figura C.2). Isso é observado os picos levemente deslocados para a esquerda, quando comparamos o grupo 3 com o grupo 1. Além disso, os grupo 2 e 3 apresentam um primeiro pico na $\mathrm{G}(\mathrm{r})$ com maior intensidade em relação ao grupo 1.

Por essa razão, observamos que o comprimento da caixa diminui de $37.9 \AA$ (grupo 1) para $35.6 \AA$ (grupo 3). Isso leva a um decréscimo do volume da caixa e, consequentemente, ao aumento da densidade do sistema.

Foram analisados a influência de três grupos de carga nas propriedades do sistema, quando combinados com correções de longo alcance do tipo campo de reação. O grupo 1, onde a molécula é considerada como um único grupo de carga, apresentou 


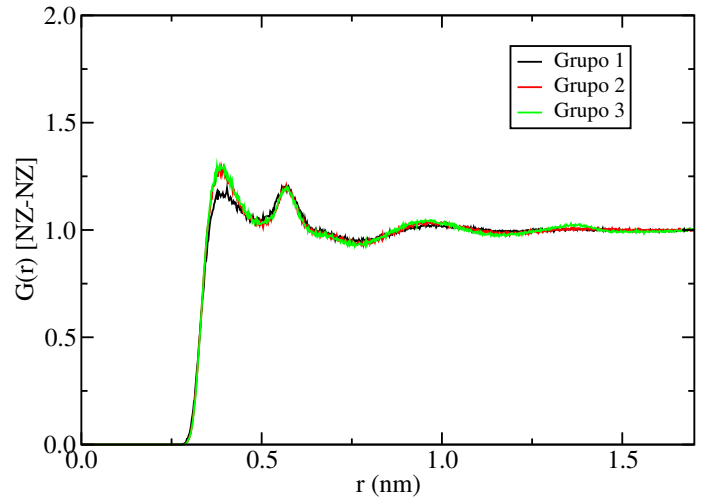

(a) NZ-NZ

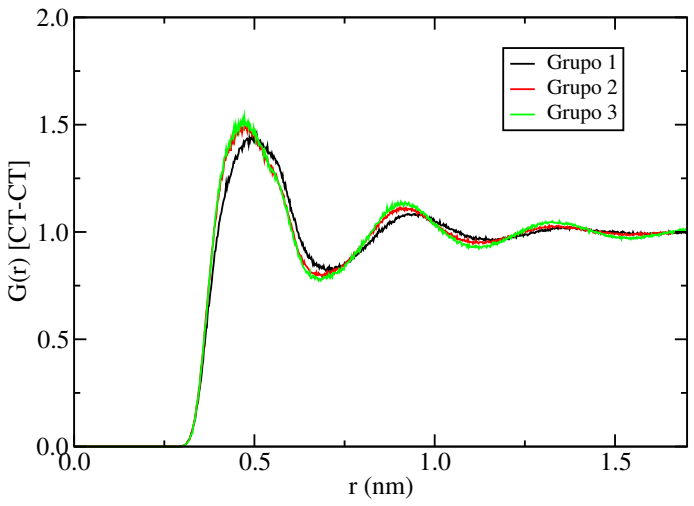

(b) CT-CT

Figura C.2: Função de distribuição radial de pares, G(r), calculadas nas dinâmicas com campo de reação como correção de longo alcance.

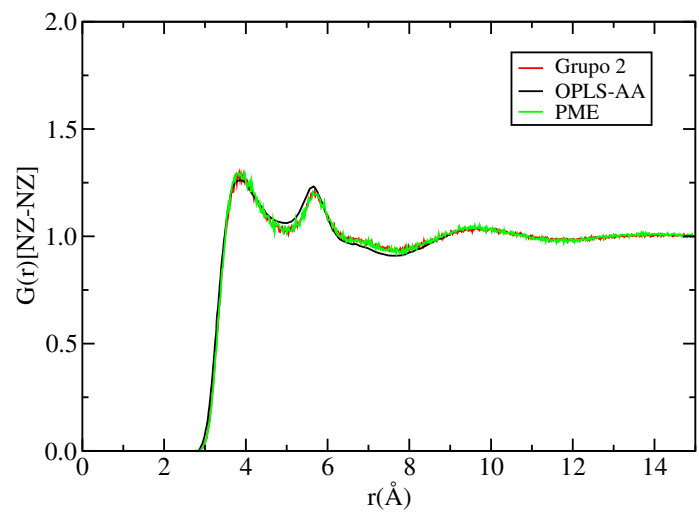

(a) NZ-NZ

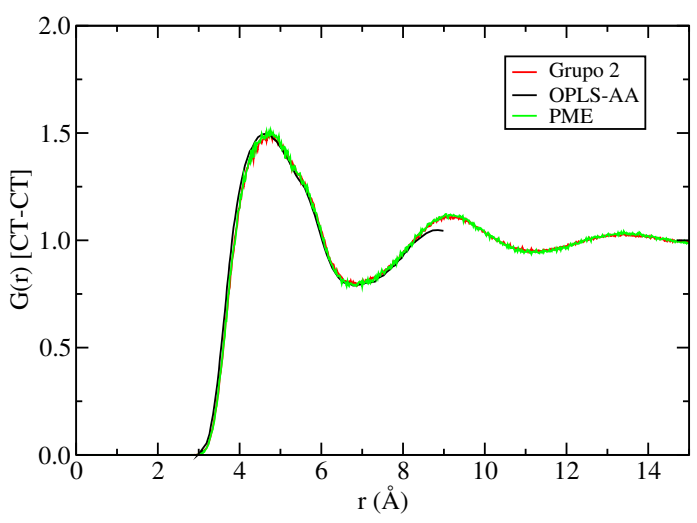

(b) CT-CT

Figura C.3: Comparação entre as G(r) calculadas neste trabalho (usando o grupo 2 com campo de reação e PME) e no trabalho original (ref. [209])

pior resultado para a densidade do sistema, $625 \mathrm{~kg} / \mathrm{m}^{3}$, embora seja a única forma de definir um grupo de carga nula. O grupo 3, onde a molécula foi separada em 3 grupos, apresentou melhor resultado para a densidade, $751 \mathrm{~kg} / \mathrm{m}^{3}$, mas os grupos ficaram com cargas não nula, portanto não é recomendado usá-los. Finalmente, o grupo 2, com dois grupos de carga de 0.1 e -0.1, apresentou um melhor equilíbrio com densidade de $735 \mathrm{~kg} / \mathrm{m}^{3}$, e é a melhor escolha caso seja necessário usar esse tipo de correção de longo alcance. 
Mas os melhores resultados foram obtidos usando PME. A densidade do sistema foi de $744.8 \pm 0.8 \mathrm{~g} / \mathrm{cm}^{3}$. A distribuição radial também está em bom acordo com os resultados originais do campo de força (ver Figura C.3). Por essa razão, esse método foi empregado nas dinâmicas ao longo desse trabalho. 


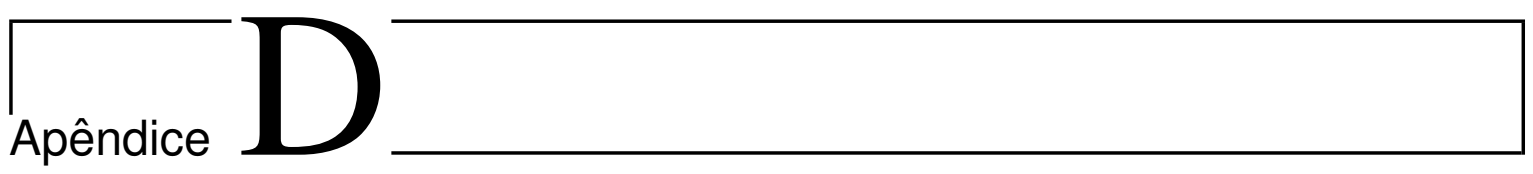

\section{Arquivos adicionais das dinâmicas}

\section{D.1 C2PK}

Exemplo de arquivo de entrada do CP2K, usado para a dinâmica molecular por primeiros princípios.

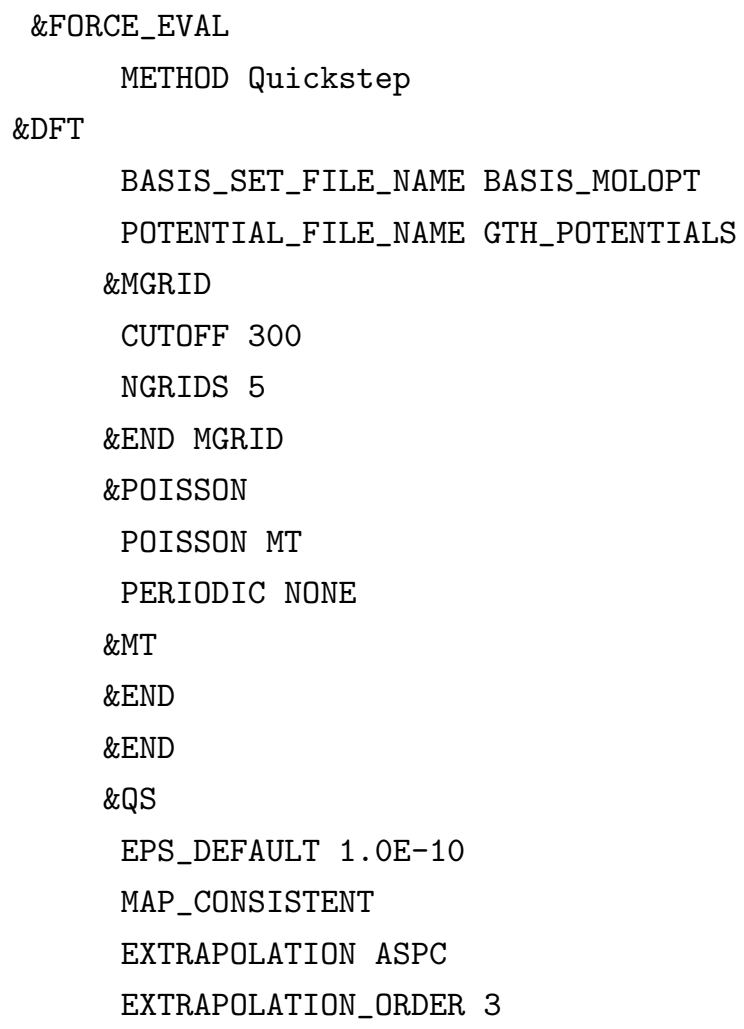




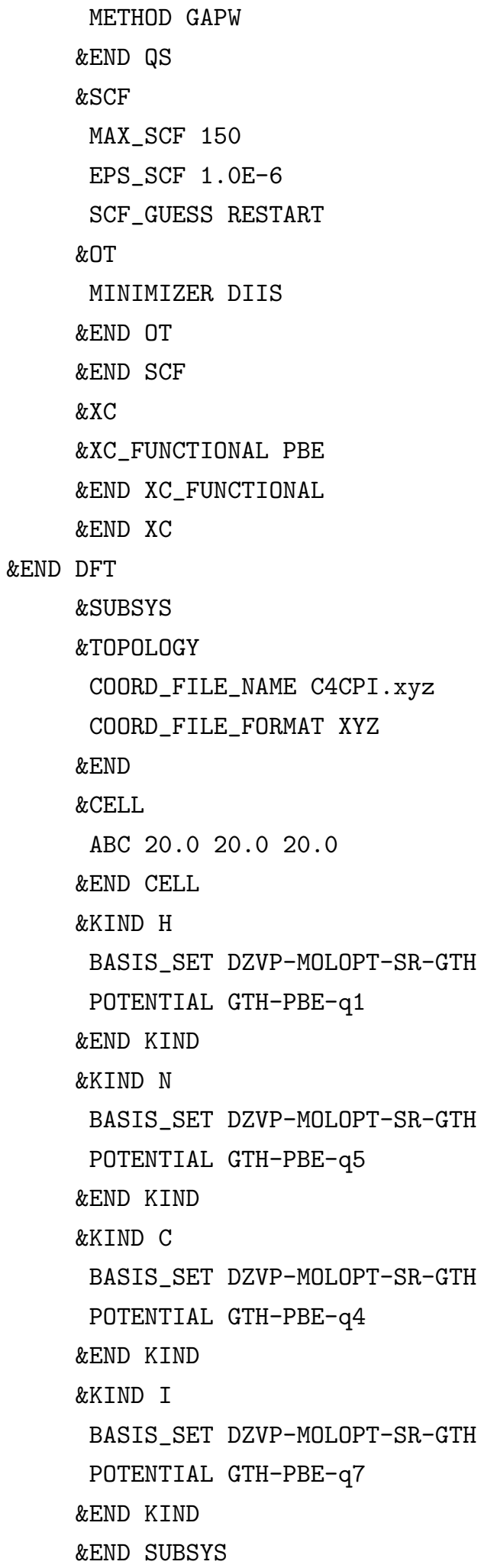


\&END FORCE_EVAL

$\&$ GLOBAL

PROJECT PYR

RUN_TYPE MD

PRINT_LEVEL LOW

WALLTIME 86000

\&END GLOBAL

\&MOTION

\&MD

ENSEMBLE NVT

STEPS 4000

TIMESTEP 0.25

TEMPERATURE 300.0

\&THERMOSTAT

TYPE CSVR

\&CSVR

TIMECON 5.0

\&END CSVR

\&END THERMOSTAT

\&END MD

\&END MOTION

\&EXT_RESTART

RESTART_FILE_NAME PYR-1.restart

RESTART_DEFAULT T

\&END EXT_RESTART 


\section{D.2 Gromacs}

Arquivo de topologia usado na dinâmica molecular clássica.

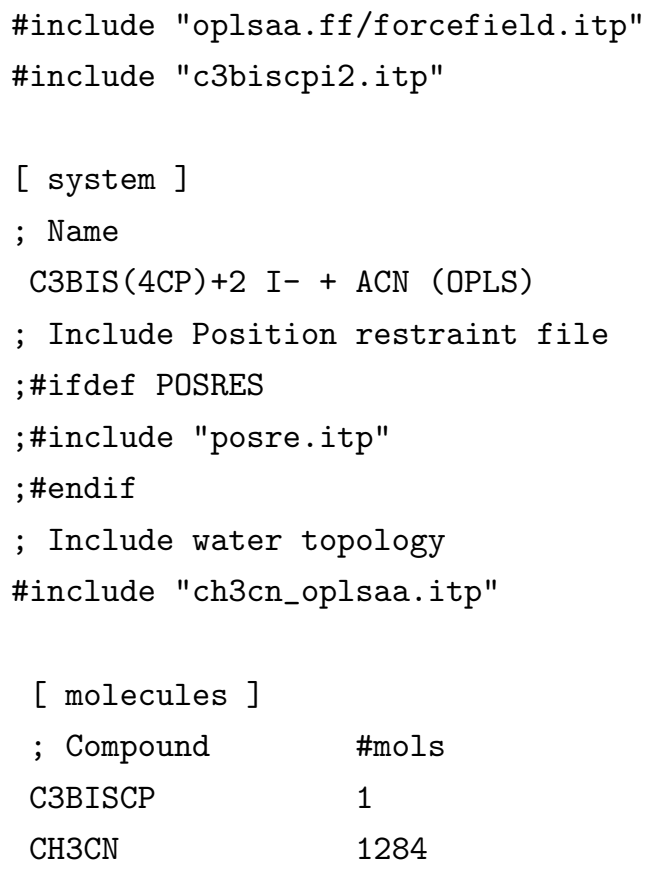

\section{D.2.1 Topologia do $C_{3} b i s(4 C P)^{2+}$ :}

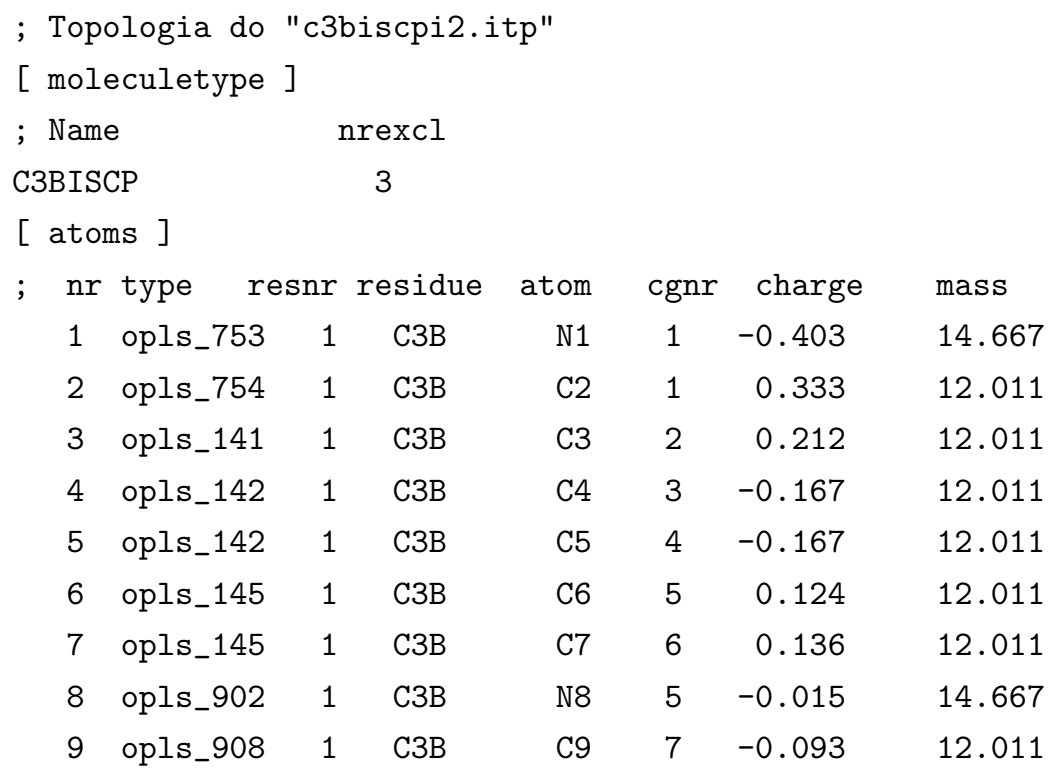




$\begin{array}{llllrrrr}10 & \text { opls_136 } & 1 & \text { C3B } & \text { C10 } & 8 & 0.186 & 12.011 \\ 11 & \text { opls_908 } & 1 & \text { C3B } & \text { C11 } & 9 & -0.159 & 12.011 \\ 12 & \text { opls_902 } & 1 & \text { C3B } & \text { N12 } & 10 & 0.162 & 14.667 \\ 13 & \text { opls_145 } & 1 & \text { C3B } & \text { C13 } & 10 & 0.015 & 12.011 \\ 14 & \text { opls_145 } & 1 & \text { C3B } & \text { C14 } & 11 & 0.008 & 12.011 \\ 15 & \text { opls_142 } & 1 & \text { C3B } & \text { C15 } & 12 & -0.086 & 12.011 \\ 16 & \text { opls_142 } & 1 & \text { C3B } & \text { C16 } & 13 & -0.074 & 12.011 \\ 17 & \text { opls_141 } & 1 & \text { C3B } & \text { C17 } & 14 & 0.108 & 12.011 \\ 18 & \text { opls_754 } & 1 & \text { C3B } & \text { C18 } & 15 & 0.357 & 12.011 \\ 19 & \text { opls_753 } & 1 & \text { C3B } & \text { N19 } & 15 & -0.407 & 14.667 \\ 20 & \text { opls_140 } & 1 & \text { C3B } & \text { H20 } & 3 & 0.169 & 1.008 \\ 21 & \text { opls_140 } & 1 & \text { C3B } & \text { H21 } & 4 & 0.196 & 1.008 \\ 22 & \text { opls_140 } & 1 & \text { C3B } & \text { H22 } & 5 & 0.173 & 1.008 \\ 23 & \text { opls_140 } & 1 & \text { C3B } & \text { H23 } & 6 & 0.155 & 1.008 \\ 24 & \text { opls_140 } & 1 & \text { C3B } & \text { H24 } & 7 & 0.111 & 1.008 \\ 25 & \text { opls_140 } & 1 & \text { C3B } & \text { H25 } & 7 & 0.134 & 1.008 \\ 26 & \text { opls_140 } & 1 & \text { C3B } & \text { H26 } & 8 & 0.027 & 1.008 \\ 27 & \text { opls_140 } & 1 & \text { C3B } & \text { H27 } & 8 & 0.034 & 1.008 \\ 28 & \text { opls_140 } & 1 & \text { C3B } & \text { H28 } & 9 & 0.115 & 1.008 \\ 29 & \text { opls_140 } & 1 & \text { C3B } & \text { H29 } & 9 & 0.101 & 1.008 \\ 30 & \text { opls_140 } & 1 & \text { C3B } & \text { H30 } & 10 & 0.195 & 1.008 \\ 31 & \text { opls_140 } & 1 & \text { C3B } & \text { H31 } & 11 & 0.193 & 1.008 \\ 32 & \text { opls_140 } & 1 & \text { C3B } & \text { H32 } & 12 & 0.164 & 1.008 \\ 33 & \text { opls_140 } & 1 & \text { C3B } & \text { H33 } & 13 & 0.163 & 1.008 \\ 34 & \text { opls_732 } & 2 & \text { I- } & \text { I34 } & 14 & -1.000 & 126.9045 \\ 35 & \text { opls_732 } & 3 & \text { I- } & \text { I35 } & 15 & -1.000 & 126.9045\end{array}$

[ bonds ]

; ai aj funct

$\begin{array}{rrrlllll}1 & 2 & 1 & 0.11700 & 543920.0 & \text {; wlj 9/98 } & \text { CZ } & \text { NZ } \\ 2 & 3 & 1 & 0.14360 & 334720.0 & \text {; wlj 9/98 } & \text { CA } & \text { CZ } \\ 3 & 4 & 1 & 0.14210 & 392459.2 & \text {; TRP, TYR,PHE CA } & \text { CA } \\ 3 & 5 & 1 & 0.14047 & 392459.2 & \text {; TRP, TYR,PHE CA } & \text { CA } \\ 4 & 6 & 1 & 0.13850 & 392459.2 & \text {; TRP, TYR,PHE CA } & \text { CA } \\ 4 & 20 & 1 & 0.10820 & 307105.6 & \text {; PHE, etc. } & \text { CA } & \text { HA } \\ 5 & 7 & 1 & 0.14050 & 392459.2 & \text {; TRP, TYR,PHE CA } & \text { CA } \\ 5 & 21 & 1 & 0.10820 & 307105.6 & \text {; PHE, etc. } & \text { CA } & \text { HA } \\ 6 & 8 & 1 & 0.13740 & 404174.4 & \text {; wlj } & \text { C! } & \text { NC } \\ 6 & 22 & 1 & 0.10820 & 307105.6 & \text {; PHE, etc. } & \text { CA } & \text { HA } \\ 7 & 8 & 1 & 0.13690 & 404174.4 & \text {; wlj } & \text { C! } & \text { NC } \\ 7 & 23 & 1 & 0.10820 & 307105.6 & \text {; PHE, etc. } & \text { CA } & \text { HA }\end{array}$




$\begin{array}{rrrrrlll}8 & 9 & 1 & 0.15010 & 392459.2 & \text {; TRP, TYR, PHE CA } & \text { CA } \\ 9 & 10 & 1 & 0.15430 & 224262.4 & \text {; CHARMM 22 } & \text { CT } & \text { CT } \\ 9 & 24 & 1 & 0.10959 & 284512.0 & \text {; wlj 7/96 } & \text { HC } & \text { C } \\ 9 & 25 & 1 & 0.10959 & 284512.0 & \text {; wlj 7/96 } & \text { HC } & \text { C } \\ 10 & 11 & 1 & 0.15410 & 224262.4 & \text {; CHARMM 22 } & \text { CT } & \text { CT } \\ 10 & 26 & 1 & 0.10959 & 284512.0 & \text {; wlj 7/96 } & \text { HC } & \text { C } \\ 10 & 27 & 1 & 0.10959 & 284512.0 & \text {; wlj 7/96 } & \text { HC } & \text { C } \\ 11 & 12 & 1 & 0.14840 & 392459.2 & \text {; TRP, TYR,PHE CA } & \text { CA } \\ 11 & 28 & 1 & 0.10959 & 284512.0 & \text {; wlj 7/96 } & \text { HC } & \text { C } \\ 11 & 29 & 1 & 0.10959 & 284512.0 & \text {; wlj 7/96 } & \text { HC } & \text { C } \\ 12 & 13 & 1 & 0.13690 & 404174.4 & \text {; wlj } & \text { C! } & \text { NC } \\ 12 & 14 & 1 & 0.13700 & 404174.4 & \text {; wlj } & \text { C! } & \text { NC } \\ 13 & 15 & 1 & 0.13890 & 392459.2 & \text {; TRP, TYR,PHE CA } & \text { CA } \\ 13 & 30 & 1 & 0.10820 & 307105.6 & \text {; PHE, etc. } & \text { CA } & \text { HA } \\ 14 & 16 & 1 & 0.13880 & 392459.2 & \text {; TRP, TYR,PHE CA } & \text { CA } \\ 14 & 31 & 1 & 0.10820 & 307105.6 & \text {; PHE, etc. } & \text { CA } & \text { HA } \\ 15 & 17 & 1 & 0.14180 & 392459.2 & \text {; TRP, TYR,PHE CA } & \text { CA } \\ 15 & 32 & 1 & 0.10820 & 307105.6 & \text {; PHE, etc. } & \text { CA } & \text { HA } \\ 16 & 17 & 1 & 0.14150 & 392459.2 & \text {; TRP, TYR,PHE CA } & \text { CA } \\ 16 & 33 & 1 & 0.10820 & 307105.6 & \text {; PHE, etc. } & \text { CA } & \text { HA } \\ 17 & 18 & 1 & 0.14330 & 334720.0 & \text {; wlj 9/98 } & \text { CA } & \text { CZ } \\ 18 & 19 & 1 & 0.11730 & 543920.0 & \text {; wlj 9/98 } & \text { CZ } & \text { NZ }\end{array}$

[ angles ]

$\begin{array}{rrrrrrrrr}\text {; } & j & \mathrm{k} & \text { func } & \text { th0 } & \text { cth } & & & \\ 1 & 2 & 3 & 1 & 179.650 & 1255.200 & \text {;CA } & \text { CZ } & \text { NZ } \\ 2 & 3 & 4 & 1 & 120.738 & 527.184 & \text {;CA } & \text { CA } & \text { CR } \\ 2 & 3 & 5 & 1 & 120.738 & 527.184 & \text {;CA } & \text { CA } & \text { CR } \\ 3 & 4 & 6 & 1 & 119.555 & 527.184 & \text {;CA } & \text { CA } & \text { CR } \\ 3 & 4 & 20 & 1 & 121.296 & 527.184 & \text {;CA } & \text { CA } & \text { CR } \\ 3 & 5 & 7 & 1 & 119.139 & 527.184 & \text {;CA } & \text { CA } & \text { CR } \\ 3 & 5 & 21 & 1 & 121.112 & 292.880 & \text {;CA } & \text { CA } & \text { HA } \\ 4 & 3 & 5 & 1 & 118.958 & 292.880 & \text {;CA } & \text { CA } & \text { HA } \\ 4 & 6 & 8 & 1 & 120.395 & 585.760 & \text {;CA } & \text { CA } & \text { NC } \\ 4 & 6 & 22 & 1 & 123.421 & 292.880 & \text {;CA } & \text { CA } & \text { HA } \\ 5 & 7 & 8 & 1 & 121.013 & 585.760 & \text {;CA } & \text { CA } & \text { NC } \\ 5 & 7 & 23 & 1 & 122.345 & 292.880 & \text {;CA } & \text { CA } & \text { HA } \\ 6 & 4 & 20 & 1 & 121.296 & 292.880 & \text {;CA } & \text { CA } & \text { HA } \\ 6 & 8 & 7 & 1 & 120.915 & 585.760 & \text {;CA } & \text { CA } & \text { NC } \\ 6 & 8 & 9 & 1 & 118.550 & 585.760 & \text {;CA } & \text { CA } & \text { NC } \\ 7 & 5 & 21 & 1 & 119.767 & 292.880 & \text {;CA } & \text { CA } & \text { HA }\end{array}$




\begin{tabular}{|c|c|c|c|c|c|c|c|c|}
\hline 7 & 8 & 9 & 1 & 120.515 & 585.760 & ; CA & $\mathrm{CA}$ & $\mathrm{NC}$ \\
\hline 8 & 6 & 22 & 1 & 116.156 & 292.880 & ; NC & $\mathrm{CA}$ & $\mathrm{HA}$ \\
\hline 8 & 7 & 23 & 1 & 116.641 & 292.880 & ; NC & $\mathrm{CA}$ & $\mathrm{HA}$ \\
\hline 8 & 9 & 10 & 1 & 112.280 & 488.273 & ; CT & $\mathrm{CT}$ & $\mathrm{CT}$ \\
\hline 8 & 9 & 24 & 1 & 106.287 & 313.800 & ;CT & $\mathrm{CT}$ & $\mathrm{HC}$ \\
\hline 8 & 9 & 25 & 1 & 107.148 & 313.800 & ; CT & $\mathrm{CT}$ & $\mathrm{HC}$ \\
\hline 9 & 10 & 11 & 1 & 108.840 & 488.273 & ; CT & $\mathrm{CT}$ & CT \\
\hline 9 & 10 & 26 & 1 & 108.227 & 313.800 & ; CT & $\mathrm{CT}$ & $\mathrm{HC}$ \\
\hline 9 & 10 & 27 & 1 & 111.413 & 313.800 & ;CT & $\mathrm{CT}$ & $\mathrm{HC}$ \\
\hline 10 & 9 & 24 & 1 & 108.907 & 313.800 & ; CT & $\mathrm{CT}$ & $\mathrm{HC}$ \\
\hline 10 & 9 & 25 & 1 & 112.276 & 313.800 & ; CT & $\mathrm{CT}$ & $\mathrm{HC}$ \\
\hline 10 & 11 & 12 & 1 & 112.280 & 488.273 & ; CT & $\mathrm{CT}$ & $\mathrm{CT}$ \\
\hline 10 & 11 & 28 & 1 & 108.907 & 313.800 & ;CT & $\mathrm{CT}$ & $\mathrm{HC}$ \\
\hline 10 & 11 & 29 & 1 & 112.276 & 313.800 & ; CT & CT & $\mathrm{HC}$ \\
\hline 11 & 10 & 26 & 1 & 108.227 & 313.800 & ; CT & $\mathrm{CT}$ & $\mathrm{HC}$ \\
\hline 11 & 10 & 27 & 1 & 111.413 & 313.800 & ; CT & $\mathrm{CT}$ & $\mathrm{HC}$ \\
\hline 11 & 12 & 13 & 1 & 118.550 & 585.760 & ;CA & $\mathrm{CA}$ & $\mathrm{NC}$ \\
\hline 11 & 12 & 14 & 1 & 120.515 & 585.760 & ;CA & $\mathrm{CA}$ & $\mathrm{NC}$ \\
\hline 12 & 11 & 28 & 1 & 106.287 & 292.880 & ; NC & $\mathrm{CA}$ & HA \\
\hline 12 & 11 & 29 & 1 & 107.148 & 292.880 & ; NC & $\mathrm{CA}$ & $\mathrm{HA}$ \\
\hline 12 & 13 & 15 & 1 & 120.395 & 585.760 & ;CA & $\mathrm{CA}$ & $\mathrm{NC}$ \\
\hline 12 & 13 & 30 & 1 & 116.156 & 292.880 & ; NC & $\mathrm{CA}$ & HA \\
\hline 12 & 14 & 16 & 1 & 121.013 & 585.760 & ; CA & $\mathrm{CA}$ & $\mathrm{NC}$ \\
\hline 12 & 14 & 31 & 1 & 116.641 & 292.880 & ; NC & $\mathrm{CA}$ & HA \\
\hline 13 & 12 & 14 & 1 & 120.915 & 585.760 & ; CA & $\mathrm{CA}$ & NC \\
\hline 13 & 15 & 17 & 1 & 119.577 & 527.184 & ; CA & $\mathrm{CA}$ & $\mathrm{CR}$ \\
\hline 13 & 15 & 32 & 1 & 119.127 & 292.880 & ; CA & $\mathrm{CA}$ & HA \\
\hline 14 & 16 & 17 & 1 & 119.139 & 527.184 & ; CA & $\mathrm{CA}$ & $\mathrm{CR}$ \\
\hline 14 & 16 & 33 & 1 & 119.728 & 292.880 & ;CA & $\mathrm{CA}$ & HA \\
\hline 15 & 13 & 30 & 1 & 123.421 & 292.880 & ; CA & $\mathrm{CA}$ & HA \\
\hline 15 & 17 & 16 & 1 & 118.958 & 527.184 & ;CA & $\mathrm{CA}$ & $\mathrm{CR}$ \\
\hline 15 & 17 & 18 & 1 & 120.742 & 527.184 & ;CA & $\mathrm{CA}$ & $\mathrm{CR}$ \\
\hline 16 & 14 & 31 & 1 & 122.345 & 292.880 & ; CA & $\mathrm{CA}$ & HA \\
\hline 16 & 17 & 18 & 1 & 120.301 & 527.184 & ; CA & $\mathrm{CA}$ & $\mathrm{CR}$ \\
\hline 17 & 16 & 33 & 1 & 121.133 & 292.880 & ;CA & $\mathrm{CA}$ & $\mathrm{HA}$ \\
\hline 17 & 15 & 32 & 1 & 121.296 & 292.880 & ; CA & $\mathrm{CA}$ & HA \\
\hline 17 & 18 & 19 & 1 & 179.650 & 1255.200 & ; CA & $\mathrm{CZ}$ & NZ \\
\hline 24 & 9 & 25 & 1 & 109.753 & 276.144 & ; HC & $\mathrm{CT}$ & $\mathrm{HC}$ \\
\hline 26 & 10 & 27 & 1 & 108.619 & 276.144 & ; HC & $\mathrm{CT}$ & $\mathrm{HC}$ \\
\hline 28 & 11 & 29 & 1 & 109.753 & 276.144 & ; HC & $\mathrm{CT}$ & $\mathrm{HC}$ \\
\hline
\end{tabular}


[ dihedrals ]

$\begin{array}{rrrrrrrrr}\text {;ai } & \text { aj } & \text { ak } & \text { al funct } & \text { c0 } & \text { c1 } & c 2 & c 3 \\ 2 & 3 & 4 & 6 & 3 & 30.33400 & 0.00000 & -30.33400 & 0.00000 \\ 2 & 3 & 4 & 20 & 3 & 30.33400 & 0.00000 & -30.33400 & 0.00000 \\ 5 & 3 & 4 & 6 & 3 & 30.33400 & 0.00000 & -30.33400 & 0.00000 \\ 5 & 3 & 4 & 20 & 3 & 30.33400 & 0.00000 & -30.33400 & 0.00000 \\ 2 & 3 & 5 & 7 & 3 & 30.33400 & 0.00000 & -30.33400 & 0.00000 \\ 2 & 3 & 5 & 21 & 3 & 30.33400 & 0.00000 & -30.33400 & 0.00000 \\ 4 & 3 & 5 & 7 & 3 & 30.33400 & 0.00000 & -30.33400 & 0.00000 \\ 4 & 3 & 5 & 21 & 3 & 30.33400 & 0.00000 & -30.33400 & 0.00000 \\ 3 & 4 & 6 & 8 & 3 & 30.33400 & 0.00000 & -30.33400 & 0.00000 \\ 3 & 4 & 6 & 22 & 3 & 30.33400 & 0.00000 & -30.33400 & 0.00000 \\ 20 & 4 & 6 & 8 & 3 & 30.33400 & 0.00000 & -30.33400 & 0.00000 \\ 20 & 4 & 6 & 22 & 3 & 30.33400 & 0.00000 & -30.33400 & 0.00000 \\ 3 & 5 & 7 & 8 & 3 & 30.33400 & 0.00000 & -30.33400 & 0.00000 \\ 3 & 5 & 7 & 23 & 3 & 30.33400 & 0.00000 & -30.33400 & 0.00000 \\ 21 & 5 & 7 & 8 & 3 & 30.33400 & 0.00000 & -30.33400 & 0.00000 \\ 21 & 5 & 7 & 23 & 3 & 30.33400 & 0.00000 & -30.33400 & 0.00000 \\ 4 & 6 & 8 & 7 & 3 & 30.33400 & 0.00000 & -30.33400 & 0.00000 \\ 4 & 6 & 8 & 9 & 3 & 30.33400 & 0.00000 & -30.33400 & 0.00000 \\ 22 & 6 & 8 & 7 & 3 & 30.33400 & 0.00000 & -30.33400 & 0.00000 \\ 22 & 6 & 8 & 9 & 3 & 30.33400 & 0.00000 & -30.33400 & 0.00000 \\ 5 & 7 & 8 & 6 & 3 & 30.33400 & 0.00000 & -30.33400 & 0.00000 \\ 5 & 7 & 8 & 9 & 3 & 30.33400 & 0.00000 & -30.33400 & 0.00000 \\ 23 & 7 & 8 & 6 & 3 & 30.33400 & 0.00000 & -30.33400 & 0.00000 \\ 23 & 7 & 8 & 9 & 3 & 30.33400 & 0.00000 & -30.33400 & 0.00000 \\ 6 & 8 & 9 & 10 & 3 & 0.00000 & 0.00000 & 0.00000 & 0.00000 \\ 6 & 8 & 9 & 24 & 3 & 0.00000 & 0.00000 & 0.00000 & 0.00000 \\ 6 & 8 & 9 & 25 & 3 & 0.00000 & 0.00000 & 0.00000 & 0.00000 \\ 7 & 8 & 9 & 10 & 3 & 0.00000 & 0.00000 & 0.00000 & 0.00000 \\ 7 & 8 & 9 & 24 & 3 & 0.00000 & 0.00000 & 0.00000 & 0.00000 \\ 7 & 8 & 9 & 25 & 3 & 0.00000 & 0.00000 & 0.00000 & 0.00000 \\ 8 & 9 & 10 & 11 & 3 & 2.92880 & -1.46440 & 0.20920 & -1.67360 \\ 8 & 9 & 10 & 26 & 3 & 0.62760 & 1.88280 & 0.00000 & -2.51040 \\ 8 & 9 & 10 & 27 & 3 & 0.62760 & 1.88280 & 0.00000 & -2.51040 \\ 24 & 9 & 10 & 11 & 3 & 0.62760 & 1.88280 & 0.00000 & -2.51040 \\ 24 & 9 & 10 & 26 & 3 & 0.62760 & 1.88280 & 0.00000 & -2.51040 \\ 24 & 9 & 10 & 27 & 3 & 0.62760 & 1.88280 & 0.00000 & -2.51040 \\ 25 & 9 & 10 & 11 & 3 & 0.62760 & 1.88280 & 0.00000 & -2.51040 \\ 25 & 9 & 10 & 26 & 3 & 0.62760 & 1.88280 & 0.00000 & -2.51040\end{array}$




\begin{tabular}{|c|c|c|c|c|c|c|c|c|}
\hline 25 & 9 & 10 & 27 & 3 & 0.62760 & 1.88280 & 0.00000 & -2.51040 \\
\hline 9 & 10 & 11 & 12 & 3 & 2.92880 & -1.46440 & 0.20920 & -1.67360 \\
\hline 9 & 10 & 11 & 28 & 3 & 0.62760 & 1.88280 & 0.00000 & -2.51040 \\
\hline 9 & 10 & 11 & 29 & 3 & 0.62760 & 1.88280 & 0.00000 & -2.51040 \\
\hline 26 & 10 & 11 & 12 & 3 & 0.62760 & 1.88280 & 0.00000 & -2.51040 \\
\hline 26 & 10 & 11 & 28 & 3 & 0.62760 & 1.88280 & 0.00000 & -2.51040 \\
\hline 26 & 10 & 11 & 29 & 3 & 0.62760 & 1.88280 & 0.00000 & -2.51040 \\
\hline 27 & 10 & 11 & 12 & 3 & 0.62760 & 1.88280 & 0.00000 & -2.51040 \\
\hline 27 & 10 & 11 & 28 & 3 & 0.62760 & 1.88280 & 0.00000 & -2.51040 \\
\hline 27 & 10 & 11 & 29 & 3 & 0.62760 & 1.88280 & 0.00000 & -2.51040 \\
\hline 10 & 11 & 12 & 13 & 3 & 0.00000 & 0.00000 & 0.00000 & 0.00000 \\
\hline 10 & 11 & 12 & 14 & 3 & 0.00000 & 0.00000 & 0.00000 & 0.00000 \\
\hline 28 & 11 & 12 & 13 & 3 & 0.00000 & 0.00000 & 0.00000 & 0.00000 \\
\hline 28 & 11 & 12 & 14 & 3 & 0.00000 & 0.00000 & 0.00000 & 0.00000 \\
\hline 29 & 11 & 12 & 13 & 3 & 0.00000 & 0.00000 & 0.00000 & 0.00000 \\
\hline 29 & 11 & 12 & 14 & 3 & 0.00000 & 0.00000 & 0.00000 & 0.00000 \\
\hline 11 & 12 & 13 & 15 & 3 & 30.33400 & 0.00000 & -30.33400 & 0.00000 \\
\hline 11 & 12 & 13 & 30 & 3 & 30.33400 & 0.00000 & -30.33400 & 0.00000 \\
\hline 14 & 12 & 13 & 15 & 3 & 30.33400 & 0.00000 & -30.33400 & 0.00000 \\
\hline 14 & 12 & 13 & 30 & 3 & 30.33400 & 0.00000 & -30.33400 & 0.00000 \\
\hline 11 & 12 & 14 & 16 & 3 & 30.33400 & 0.00000 & -30.33400 & 0.00000 \\
\hline 11 & 12 & 14 & 31 & 3 & 30.33400 & 0.00000 & -30.33400 & 0.00000 \\
\hline 13 & 12 & 14 & 16 & 3 & 30.33400 & 0.00000 & -30.33400 & 0.00000 \\
\hline 13 & 12 & 14 & 31 & 3 & 30.33400 & 0.00000 & -30.33400 & 0.00000 \\
\hline 12 & 13 & 15 & 17 & 3 & 30.33400 & 0.00000 & -30.33400 & 0.00000 \\
\hline 12 & 13 & 15 & 32 & 3 & 30.33400 & 0.00000 & -30.33400 & 0.00000 \\
\hline 30 & 13 & 15 & 17 & 3 & 30.33400 & 0.00000 & -30.33400 & 0.00000 \\
\hline 30 & 13 & 15 & 32 & 3 & 30.33400 & 0.00000 & -30.33400 & 0.00000 \\
\hline 12 & 14 & 16 & 17 & 3 & 30.33400 & 0.00000 & -30.33400 & 0.00000 \\
\hline 12 & 14 & 16 & 33 & 3 & 30.33400 & 0.00000 & -30.33400 & 0.00000 \\
\hline 31 & 14 & 16 & 17 & 3 & 30.33400 & 0.00000 & -30.33400 & 0.00000 \\
\hline 31 & 14 & 16 & 33 & 3 & 30.33400 & 0.00000 & -30.33400 & 0.00000 \\
\hline 13 & 15 & 17 & 16 & 3 & 30.33400 & 0.00000 & -30.33400 & 0.00000 \\
\hline 13 & 15 & 17 & 18 & 3 & 30.33400 & 0.00000 & -30.33400 & 0.00000 \\
\hline 32 & 15 & 17 & 16 & 3 & 30.33400 & 0.00000 & -30.33400 & 0.00000 \\
\hline 32 & 15 & 17 & 18 & 3 & 30.33400 & 0.00000 & -30.33400 & 0.00000 \\
\hline 14 & 16 & 17 & 15 & 3 & 30.33400 & 0.00000 & -30.33400 & 0.00000 \\
\hline 14 & 16 & 17 & 18 & 3 & 30.33400 & 0.00000 & -30.33400 & 0.00000 \\
\hline 33 & 16 & 17 & 15 & 3 & 30.33400 & 0.00000 & -30.33400 & 0.00000 \\
\hline 33 & 16 & 17 & 18 & 3 & 30.33400 & 0.00000 & -30.33400 & 0.00000 \\
\hline
\end{tabular}


[ dihedrals ]

; improper_Z_CA_X_Y

$\begin{array}{rrrrrrrr}2 & 4 & 3 & 5 & 1 & 180 & 4.6 & 2 \\ 3 & 6 & 4 & 20 & 1 & 180 & 4.6 & 2 \\ 3 & 7 & 5 & 21 & 1 & 180 & 4.6 & 2 \\ 4 & 8 & 6 & 22 & 1 & 180 & 4.6 & 2 \\ 5 & 8 & 7 & 23 & 1 & 180 & 4.6 & 2 \\ 6 & 7 & 8 & 9 & 1 & 180 & 4.6 & 2 \\ 11 & 12 & 13 & 14 & 1 & 180 & 4.6 & 2 \\ 12 & 15 & 13 & 30 & 1 & 180 & 4.6 & 2 \\ 12 & 16 & 14 & 31 & 1 & 180 & 4.6 & 2 \\ 13 & 17 & 15 & 32 & 1 & 180 & 4.6 & 2 \\ 14 & 17 & 16 & 33 & 1 & 180 & 4.6 & 2 \\ 16 & 15 & 17 & 18 & 1 & 180 & 4.6 & 2\end{array}$

[ pairs ]

$\begin{array}{rrr}\text { ai } & \text { aj } & \text { funct } \\ 1 & 4 & 1 \\ 1 & 5 & 1 \\ 2 & 6 & 1 \\ 2 & 7 & 1 \\ 2 & 20 & 1 \\ 2 & 21 & 1 \\ 3 & 8 & 1 \\ 3 & 22 & 1 \\ 3 & 23 & 1 \\ 4 & 7 & 1 \\ 4 & 9 & 1 \\ 4 & 21 & 1 \\ 5 & 9 & 1 \\ 5 & 6 & 1 \\ 5 & 20 & 1 \\ 6 & 10 & 1 \\ 6 & 23 & 1 \\ 6 & 24 & 1 \\ 6 & 25 & 1 \\ 7 & 10 & 1 \\ 7 & 22 & 1 \\ 7 & 24 & 1 \\ 7 & 25 & 1 \\ 8 & 11 & 1\end{array}$




$\begin{array}{rll}8 & 20 & 1 \\ 8 & 21 & 1 \\ 8 & 26 & 1 \\ 8 & 27 & 1 \\ 9 & 12 & 1 \\ 9 & 22 & 1 \\ 9 & 23 & 1 \\ 9 & 28 & 1 \\ 9 & 29 & 1 \\ 10 & 13 & 1 \\ 10 & 14 & 1 \\ 11 & 15 & 1 \\ 11 & 16 & 1 \\ 11 & 24 & 1 \\ 11 & 25 & 1 \\ 11 & 30 & 1 \\ 11 & 31 & 1 \\ 12 & 17 & 1 \\ 12 & 26 & 1 \\ 12 & 27 & 1 \\ 12 & 32 & 1 \\ 12 & 33 & 1 \\ 13 & 16 & 1 \\ 13 & 18 & 1 \\ 13 & 28 & 1 \\ 13 & 29 & 1 \\ 13 & 31 & 1 \\ 14 & 15 & 1 \\ 14 & 18 & 1 \\ 14 & 28 & 1 \\ 14 & 29 & 1 \\ 14 & 30 & 1 \\ 15 & 19 & 1 \\ 15 & 33 & 1 \\ 16 & 32 & 1 \\ 16 & 19 & 1 \\ 17 & 30 & 1 \\ 17 & 31 & 1 \\ 18 & 32 & 1 \\ 18 & 33 & 1\end{array}$




$\begin{array}{lll}20 & 22 & 1 \\ 21 & 23 & 1 \\ 24 & 26 & 1 \\ 24 & 27 & 1 \\ 25 & 26 & 1 \\ 25 & 27 & 1 \\ 26 & 28 & 1 \\ 26 & 29 & 1 \\ 27 & 28 & 1 \\ 27 & 29 & 1 \\ 30 & 32 & 1 \\ 31 & 33 & 1\end{array}$

\section{D.2.2 Topologia da acetonitrila}

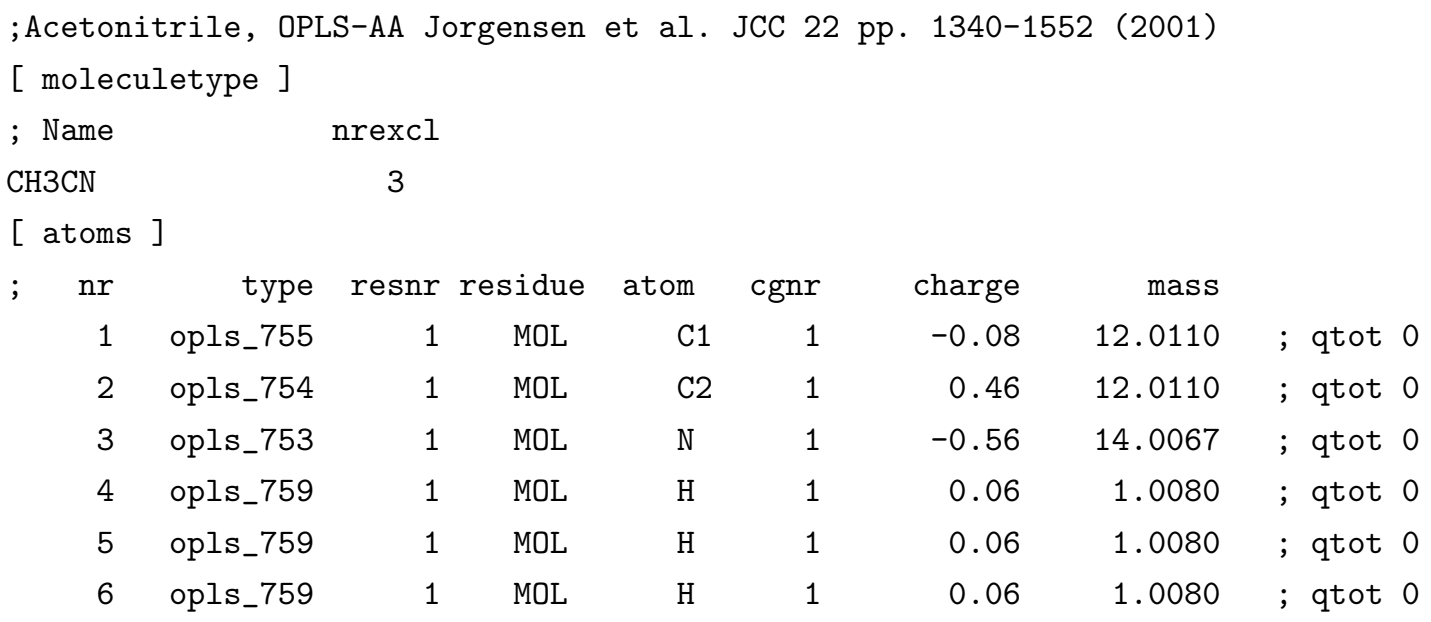

[ bonds ]

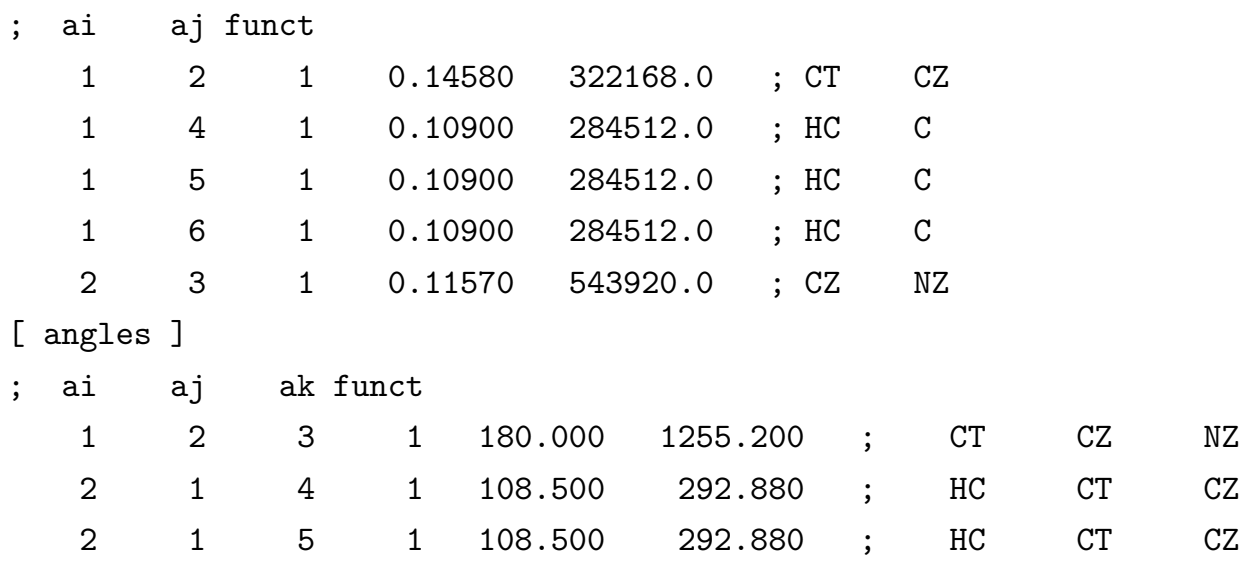




$\begin{array}{llllllllll}2 & 1 & 6 & 1 & 108.500 & 292.880 & ; & \mathrm{HC} & \mathrm{CT} & \mathrm{CZ} \\ 4 & 1 & 5 & 1 & 109.500 & 276.144 & ; & \mathrm{HC} & \mathrm{CO} & \mathrm{HC} \\ 4 & 1 & 6 & 1 & 109.500 & 276.144 & ; & \mathrm{HC} & \mathrm{CO} & \mathrm{HC} \\ 5 & 1 & 6 & 1 & 109.500 & 276.144 & ; & \mathrm{HC} & \mathrm{CO} & \mathrm{HC}\end{array}$

\section{D.2.3 Arquivo de entrada para a dinâmica clássica - GRO- MACS}

\begin{tabular}{|c|c|}
\hline integrator & $=\mathrm{md}$ \\
\hline tinit & $=0.0$ \\
\hline$d t$ & $=0.0002$ \\
\hline nsteps & $=120000000$ \\
\hline emtol & $=1$ \\
\hline nstcomm & $=5$ \\
\hline comm-grps & $=\mathrm{C} 3 \mathrm{~B} I-\mathrm{MOL}$ \\
\hline nstxout & $=10000$ \\
\hline nstvout & $=10000$ \\
\hline nstfout & $=0$ \\
\hline nstlog & $=1000$ \\
\hline nstenergy & $=1000$ \\
\hline nstxtcout & $=10000$ \\
\hline xtc_precision & $=10000$ \\
\hline xtc-grps & $=$ \\
\hline energygrps & $=\mathrm{C} 3 \mathrm{~B} I-\mathrm{MOL}$ \\
\hline nstlist & $=5$ \\
\hline ns_type & $=$ grid \\
\hline $\mathrm{pbc}$ & $=x y z$ \\
\hline rlist & $=1.4$ \\
\hline coulombtype & $=\mathrm{pme}$ \\
\hline rcoulomb & $=1.4$ \\
\hline vdw_type & $=$ cut-off \\
\hline rvdw & $=1.4$ \\
\hline DispCorr & $=\mathrm{No}$ \\
\hline tcoupl & $=\mathrm{v}$-rescale \\
\hline tc-grps & $=\mathrm{C} 3 \mathrm{~B} I-\mathrm{MOL}$ \\
\hline tau_t & $=0.10 .10 .1$ \\
\hline ref_t & $=300300300$ \\
\hline Pcoupl & $=$ berendsen \\
\hline Pcoupltype & $=$ isotropic \\
\hline
\end{tabular}




$\begin{array}{ll}\text { tau_p } & =0.5 \\ \text { compressibility } & =4.5 e-5 \\ \text { ref_p } & =1.0 \\ \text { gen-vel = yes } & \\ \text { gen-temp = 300 } & \\ \text { constraints = none } & \\ \text { constraint-algorithm = shake }\end{array}$


Tabela D.1: Cargas parciais calculadas para o complexo usando a metodologia CHELPG, considerando o átomo de iodo explicitamente (Átomo) ou como uma carga negativa (Carga). Funcional B3LYP e bases 6-311+G(d,p) para H, C, N e bases e pseudopotencial aug-ccpVDZ-PP para o iodo (raio de $2.2 \AA$ ).

\begin{tabular}{|c|c|c|c|c|}
\hline & \multicolumn{2}{|c|}{ Vácuo } & \multicolumn{2}{|c|}{ Acetonitrila (PCM) } \\
\hline & Carga & Átomo & Carga & Átomo \\
\hline N1 & -0.326 & -0.385 & -0.403 & -0.455 \\
\hline $\mathrm{C} 2$ & 0.245 & 0.326 & 0.333 & 0.389 \\
\hline C3 & 0.294 & 0.177 & 0.212 & 0.146 \\
\hline $\mathrm{C} 4$ & -0.245 & -0.232 & -0.167 & -0.149 \\
\hline C5 & -0.268 & -0.199 & -0.167 & -0.158 \\
\hline $\mathrm{C} 6$ & 0.152 & 0.246 & 0.124 & 0.136 \\
\hline $\mathrm{C} 7$ & 0.240 & 0.189 & 0.136 & 0.190 \\
\hline N8 & -0.130 & -0.178 & -0.015 & -0.096 \\
\hline C9 & -0.106 & 0.196 & -0.093 & 0.045 \\
\hline C10 & 0.301 & 0.208 & 0.186 & 0.322 \\
\hline C11 & -0.213 & 0.385 & -0.159 & 0.063 \\
\hline N12 & 0.109 & -0.051 & 0.162 & 0.039 \\
\hline $\mathrm{C} 13$ & 0.017 & 0.079 & 0.015 & 0.061 \\
\hline C14 & 0.010 & 0.190 & 0.008 & 0.086 \\
\hline $\mathrm{C} 15$ & -0.124 & -0.129 & -0.086 & -0.103 \\
\hline $\mathrm{C} 16$ & -0.111 & -0.164 & -0.074 & -0.107 \\
\hline $\mathrm{C} 17$ & 0.129 & 0.107 & 0.108 & 0.100 \\
\hline $\mathrm{C} 18$ & 0.293 & 0.326 & 0.357 & 0.394 \\
\hline N19 & -0.338 & -0.374 & -0.407 & -0.450 \\
\hline $\mathrm{H} 20$ & 0.167 & 0.138 & 0.169 & 0.138 \\
\hline $\mathrm{H} 21$ & 0.230 & 0.174 & 0.196 & 0.172 \\
\hline $\mathrm{H} 22$ & 0.184 & 0.081 & 0.173 & 0.155 \\
\hline $\mathrm{H} 23$ & 0.156 & 0.084 & 0.155 & 0.122 \\
\hline $\mathrm{H} 24$ & 0.062 & 0.019 & 0.111 & 0.041 \\
\hline $\mathrm{H} 25$ & 0.190 & -0.028 & 0.134 & 0.073 \\
\hline H26 & 0.025 & -0.087 & 0.027 & -0.050 \\
\hline $\mathrm{H} 27$ & -0.025 & -0.057 & 0.034 & -0.046 \\
\hline H28 & 0.168 & -0.085 & 0.115 & 0.051 \\
\hline $\mathrm{H} 29$ & 0.127 & -0.139 & 0.101 & -0.005 \\
\hline H30 & 0.236 & 0.117 & 0.195 & 0.158 \\
\hline H31 & 0.245 & 0.028 & 0.193 & 0.144 \\
\hline H32 & 0.153 & 0.142 & 0.164 & 0.142 \\
\hline H33 & 0.153 & 0.152 & 0.163 & 0.142 \\
\hline I34 & -1.000 & -0.627 & -1.000 & -0.845 \\
\hline I35 & -1.000 & -0.629 & -1.000 & -0.845 \\
\hline
\end{tabular}


